\title{
Large Eddy Simulationen von isolierten Scheibengalaxien
}

\section{Dissertation}

zur Erlangung des mathematisch-naturwissenschaftlichen Doktorgrades

\section{"Doctor rerum naturalium"}

der Georg-August-Universität Göttingen

im Promotionsprogramm ProPhys

der Georg-August-University School of Science (GAUSS)

vorgelegt von

\section{Harald Udo Braun}

aus Kassel

Göttingen, 2014

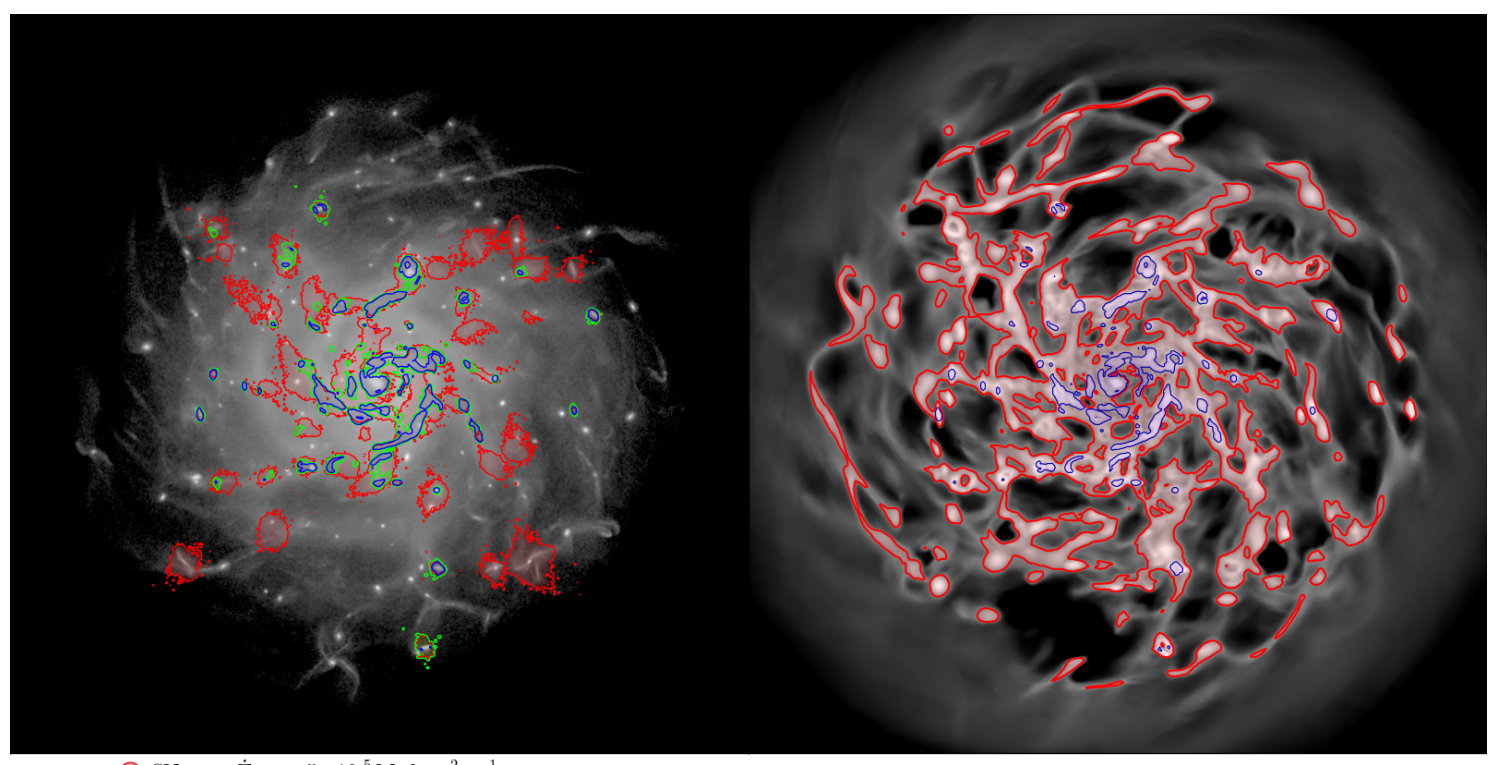

SNe rate $\dot{\Sigma}_{\mathrm{FB}}>5 \times 10^{5} \mathrm{M}_{\odot} \mathrm{kpc}^{-2} \mathrm{yr}^{-1}$

Lyman continuum photons $\dot{n}_{\mathrm{Lyc}}>10^{6} \mathrm{~cm}^{-2} \mathrm{~s}^{-1}$

$\bigcirc$ star formation rate $\dot{\Sigma}_{\mathrm{SF}}>10^{-2} \mathrm{M}_{\odot} \mathrm{kpc}^{-2} \mathrm{yr}^{-1}$

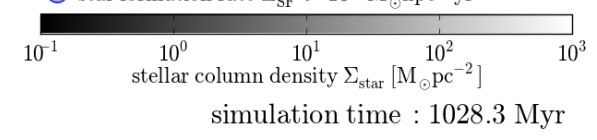

$\mathrm{HI}$ column density $\Sigma_{\mathrm{HI}}>5 \mathrm{M}_{\odot} \mathrm{pc}^{-2}$

$\mathrm{O} \mathrm{H}_{2}$ column density $\Sigma_{\mathrm{H}_{2}}>10^{-1} \mathrm{M}_{\odot} \mathrm{pc}^{-2}$

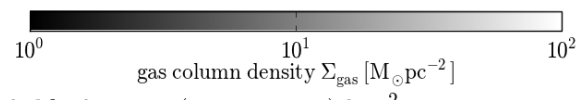

field of view : $(30.0 \times 30.0) \mathrm{kpc}^{2}$ 


\section{Betreuungsausschuss:}

Jens C. Niemeyer, Institut für Astrophysik, Universität Göttingen

Wolfram Kollatschny, Institut für Astrophysik, Universität Göttingen

Wolfram Schmidt, Institut für Astrophysik, Universität Göttingen

\section{Prüfungskomission:}

Referent:

Wolfram Schmidt, Institut für Astrophysik, Universität Göttingen

Korreferent:

Dominik Schleicher, Institut für Astrophysik, Universität Göttingen

Weitere Mitglieder der Prüfungskommission:

Stefan Dreizler, Institut für Astrophysik, Universität Göttingen

Wolfram Kollatschny, Institut für Astrophysik, Universität Göttingen

Jens C. Niemeyer, Institut für Astrophysik, Universität Göttingen

Ansgar Reiners, Institut für Astrophysik, Universität Göttingen

Tag der mündlichen Prüfung: 5. 12. 2014 
- proudly made on Earth -

- für alle, die es interessiert. 


\section{Titelbild:}

Eine isolierte Scheibengalaxie aus LES mit MIST nach ungefähr 1 Ga aus Daten der 'ref'-Simulation (Braun u. a., 2014).

Zu sehen sind in Graustufen Projektionen der stellaren Massendichte links und rechts der Gasdichte entlang Richtung der Rotationsachse. Darüber liegen farbige Konturen. Auf der linken Seite markieren die drei Konturen erstens Gebiete aktiver Sternentstehung in Blau, zweitens in Grün Bereiche mit heißen Sternen, die jünger sind als $\sim 3$ Ma und Lyman-Kontinuumstrahlung abgeben, und drittens in roter Farbe Regionen, in denen Supernovae (Typ II) möglich sind, was also die Anwesenheit von Sternenpopulationen im Alter zwischen $\sim 4 \ldots 40$ Ma anzeigt. Die Konturen auf der rechten Seite zeigen in Rot Gas an, das infolge der Separation durch thermische Instabilität in Form einer kalten und einer warmen Phase vorliegt, während Blau die Existenz von abgeschirmtem, molekularem Gas anzeigt. 


\section{Inhaltsverzeichnis}

Inhaltsverzeichnis $\quad$ i

1 Einleitung 1

2 Strömungslehre $\quad \mathbf{5}$

2.1 Grundgleichungen der Hydrodynamik . . . . . . . . . . . . . . . . . 5

2.1.1 Boltzmanngleichung . . . . . . . . . . . . . 5 5

2.1.2 Momentenbildung . . . . . . . . . . . . . 7

2.1.3 Navier-Stokes-Gleichungen . . . . . . . . . . . . . . . . . 8

2.1.4 Euler-Gleichungen . . . . . . . . . . . . . . 10

2.2 Stöße . . . . . . . . . . . . . . . . . . . . . 11

2.3 Turbulenz . . . . . . . . . . . . . . . . . . . . 13

2.3.1 Reynolds-Zahl und turbulenter Übergang . . . . . . . . . . . . 14

2.3.2 Kolmogorov Theorie . . . . . . . . . . . . . . . 15

3 Astrophysik 21

3.1 Spiralgalaxien . . . . . . . . . . . . . . . . . 21

3.2 Interstellares Medium . . . . . . . . . . . . . . . . . . 24

3.2.1 Sternentstehung . . . . . . . . . . . . . . . . 25

3.2 .2 Jeans-Instabilität . . . . . . . . . . . . . . . . . . . . . . . . . . . . .

3.2.3 Stellare Massenverteilung . . . . . . . . . . . . . . 27

3.2.4 Kennicutt-Schmidt-Relation . . . . . . . . . . . . . 28

3.2.5 Stellare Rückwirkung . . . . . . . . . . . . . . . . 30

4 Modell des turbulenten ISM $\quad 33$

4.1 Konzeption des Modells . . . . . . . . . . . . . . . . . . . 33

4.1 .1 Phasen . . . . . . . . . . . . . . . . . 34

4.1 .2 Sternentstehung . . . . . . . . . . . . . . 36

4.1.3 Stellare Rückwirkung . . . . . . . . . . . . . . . . . . 37

4.2 Modellgleichungen . . . . . . . . . . . . . . . . . 40

4.3 Gleichgewichtslösungen . . . . . . . . . . . . . . . . . . . 41

4.4 Vergleich mit Beobachtungen . . . . . . . . . . . . . . . . 46 
5 LES von Spiralgalaxien $\quad 47$

5.1 Numerische Methoden . . . . . . . . . . . . . . . . . . . . . 47

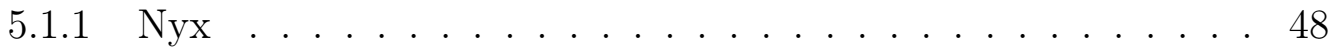

5.1 .2 SGS-Modell . . . . . . . . . . . . . . . 52

5.1 .3 MIST . . . . . . . . . . . . . . . 58

5.2 Simulationen . . . . . . . . . . . . . . . 63

5.2.1 Anfangsbedingungen . . . . . . . . . . . . . 63

5.2 .2 Ergebnisse . . . . . . . . . . . . . . . . . . 64

5.2 .3 Mögliche Erweiterungen von MIST . . . . . . . . . . . . . 71

6 Einfluß des Sternentstehungsmodells $\quad 75$

6.1 Sternentstehungsmodelle . . . . . . . . . . . . . . . . . . 75

6.1.1 KM05-Modell . . . . . . . . . . . . . . . . 77

6.1.2 HC08-13-Modell . . . . . . . . . . . . . . . . . 78

6.1.3 PN11-Modell .................... . 79

6.1 .4 PHN12-Modell . . . . . . . . . . . . . . . . 80

6.2 Simulationen . . . . . . . . . . . . . . . . . . 82

6.3 Ergebnisse . . . . . . . . . . . . . . . . . . . . . 82

6.3 .1 Sternentstehungsraten . . . . . . . . . . . . . . 82

6.3.2 Struktur der Gasscheiben . . . . . . . . . . . . . . . . . 84

6.3.3 Sternentstehungsrelationen . . . . . . . . . . . . . . . 88

6.3.4 Sternentstehung und Turbulenz . . . . . . . . . . . . . . . . . 91

6.4 Diskussion . . . . . . . . . . . . . . . . . . . . 97

6.5 Zusammenfassung . . . . . . . . . . . . . . . . . 100

$\begin{array}{lll}7 & \text { Zusammenfassung und Ausblick } & 103\end{array}$

$\begin{array}{ll}\text { Literaturverzeichnis } & 121\end{array}$

$\begin{array}{ll}\text { Danksagungen } & 123\end{array}$

A Publikationen $\quad \mathbf{1 2 5}$

A.1 Publikation Braun \& Schmidt, 2012 . . . . . . . . . . . . . . 125

A.2 Publikation Braun et al., 2014 . . . . . . . . . . . . . . . . 149 


\section{Kapitel 1}

\section{Einleitung}

\section{Der Weltraum - unendliche Weiten.}

Mit diesen Worten beginnt jede Folge der bekannten Fernsehserie 'Raumschiff Enterprise - Das Nächste Jahrhundert'. Unendlich ist die Ausdehnung des erfahrbaren Universums nach gängiger Auffassung allerdings nicht. Unfassbar groß ist jedoch die Komplexität der Strukturen und Prozesse, denen es Bühne bietet. Glücklicherweise erlaubt gerade dieser Umstand die Existenz eines derart komplexen Gebildes, wie es der Mensch ist. Dieser wurde im Laufe seiner biologischen Evolution mit Fähigkeiten ausgestattet, die es ihm ermöglichen nicht nur in seiner Umwelt zu überleben, sondern darüber hinaus noch Erkenntnisse zu gewinnen und Vermutungen über Zusammenhänge anzustellen. Er ist neugierig und entwirft Modelle (Theorien) davon, wie die Dinge ablaufen, und warum. So sind die Vorgänge am Himmel schon seit jeher Ziel der Neugier. Die gewonnenen Erkenntnisse dienen nicht der Befriedigung des akademischen Interesses allein, sondern besitzen wie die Erfindung des Kalenders auch ganz praktischen Nutzen.

Sichtweise und Erklärungsansätze für die beobachteten Phänomene haben sich mit der Zeit freilich gewandelt. So ist heute wohl niemand mehr der Auffassung, dass die Milchstraße tatsächlich aus Milch besteht, die aus der Brust der Göttin Hera bis an den Himmel spritzte. Heute wissen wir, dass die Milchstraße eine von vielen Galaxien ist. Der visuelle Eindruck eines milchig schimmernden Bandes entsteht dadurch, dass die meisten Sterne bei Betrachtung mit bloßem Auge zu etwas Flächigem verschwimmen und wir uns innerhalb einer Scheibe von einigen Milliarden Sternen befinden, von denen einer die Sonne ist. Astronomische Beobachtungen sind der einzige Hinweis und damit der Schlüssel zur Erkenntnis darüber, was sich dort draußen außerhalb unserer Reichweite abspielt. Sie können jedoch nur Momentaufnahmen liefern. Denn viele der Entwicklungsvorgänge von astrophysikalischen Objekten laufen auf Zeitskalen ab, die weit jenseits dessen sind, was innerhalb eines Menschenlebens erfahrbar wäre. Zu diesen Vorgängen gehört beispielsweise die Entstehung von Sternen aus Gaswolken, wie sie sich auch innerhalb der Milchstraße abspielt. 
Beobachtungskampagnen, die auf Erkenntnisse zu den Strukturen des Gases in nahe gelegenen Galaxien sowie zur darin ablaufenden Sternentstehung abzielen, sind zum Beispiel SINGS, THINGS, LITTLE THINGS oder PAWS (Kennicutt u.a., 2003; Walter u. a., 2008; Hunter u. a., 2012; Schinnerer u. a., 2013). Sie bedienen sich unterschiedlicher Methoden und Instrumente, um ein möglichst umfassendes Bild der Erschaffung von Sternen zu ermöglichen. Oft ist aber die Überprüfung, ob die vermuteten Mechanismen tatsächlich wirksam sind, also ob sie zu den beobachteten Strukturen und den daraus abgeleiteten statistischen Korrelationen führen können, anhand von Beobachtungen allein nicht möglich. Unter der Prämisse, dass die physikalischen Gesetzmäßigkeiten überall in gleicher Weise gelten, können Experimente diese Lücke füllen. Leider sind entsprechende apparative Experimente häufig nicht möglich oder unterliegen 'irdischen' Beschränkungen.

Mit (Hochleistungs-) Rechnern und entsprechenden Programmen lassen sich die Modelle für die mutmaßlich wirksamen Mechanismen numerisch nicht nur verifizieren oder falsifizieren, sondern auch weiterentwickeln. Sind die wesentlichen Vorgänge im Modell in korrekter Weise berücksichtigt, so lassen sich Beobachtungsergebnisse nachvollziehen - beziehungsweise sogar Ergebnisse künftiger Beobachtungen prognostizieren. Gerade, wenn es sich um Systeme mit nicht-linearem Verhalten handelt, sind Simulationen hilfreich. Zu den Problemstellungen dieser Art gehören auch Galaxien oder interstellares Gas, für deren Behandlungen eine Reihe konkurrierender Simulationsprogramme entwickelt wurden, kosmologische Hydrodynamik-Codes wie Nyx, ENZO, RAMSES, GADGET oder AREPO (Almgren u. a., 2013; O'Shea u. a., 2004; Teyssier, 2002; Springel u. a., 2001; Springel, 2010). In diese können je nach Bedarf zusätzliche Module eingebunden werden, die dann die unterschiedlichen Effekte von radiativem Kühlen, Sternentstehung, stellarer Rückwirkung und Anderem modellieren.

Das von mir betriebene Forschungsprojekt - das Thema dieser Dissertation - ist in diesem Kontext zu sehen. Es befasst sich mit der Erstellung eines effektiven Modells zur Beschreibung der Dynamik des interstellaren Mediums (ISM) auf Längenskalen von einigen zehn Parsec und seiner Untersuchung im Rahmen von Simulationen ganzer Galaxien. Zu diesem Zweck verband ich verschiedene physikalisch motivierte Ansätze zur Beschreibung einzelner Aspekte des ISM (McKee u. Ostriker, 1977; Raiteri u. a., 1996; Ferland u. a., 1998; Thornton u. a., 1998; Springel u. Hernquist, 2003; Glover u. Jappsen, 2007; Gnedin u. a., 2009; Kotulla u. a., 2009; McKee u. Krumholz, 2010; Federrath u. a., 2010; Padoan u. Nordlund, 2011, und weitere) zu einem effektiven Modell für physikalische Prozesse, die in den durchgeführten numerischen Simulationen nicht explizit aufgelöst werden können. Die wesentlichen Hilfsmittel zur Beschreibung der nicht aufgelösten Strukturen im ISM und deren Eigenschaften sind die Aufspaltung des Gasinhalts eines Referenzvolumens in zwei Fraktionen, die 
untereinander Material und Energie austauschen können, sowie die Stärke der Turbulenz im ISM, die durch die turbulente Energie parametrisiert wird. Die turbulente Energie fasst die kinetischen Energien zusammen, die ungeordneten, kleinskaligen Geschwindigkeitsfluktuationen zuzuordnen sind. Die Impulse dieser Fluktuationen veschwinden im Mittel über das Referenzvolumen. Die komplexen Strukturen im ISM werden repräsentiert durch eine dichtere, kalte Komponente und eine weniger dichte, warme, diffuse Komponente, in welche eingebettet die kalte in Form von Klumpen vorliegt.

Das von mir entwickelte Mehrphasenmodell für das ISM mit Sternentstehung und Turbulenz (kurz: MIST) ist keineswegs das erste Mehrphasenmodell für das ISM. Doch es ist einzigartig, was die Kombination von Mehrphasenmodellierung und Berücksichtigung der ISM-Turbulenz anbetrifft. Außergewöhnlich ist auch die Nutzung des vollen, dynamischen Modells innerhalb von Grobstruktursimulationen (LES; large eddy simulations) mit Kopplung an ein explizites, dynamisches SGS-Modell für kleinskalige Turbulenz (Schmidt u. Federrath, 2011; Schmidt u. a., 2014, SGS: subgrid-scale). MIST ermöglicht beispielsweise die Berechnung der lokalen Sternentstehungseffizienz bezogen auf die Freifallzeitskala aus dem aktuellen, lokalen, turbulenten Mehrphasenzustand des ISM-Gases, wo es üblich ist, einen vorab festgelegten, konstanten Wert für diese Effizienz anzunehmen (Stinson u. a., 2006; Agertz u. a., 2009; Murante u.a., 2010; Kraljic u.a., 2012; Monaco u.a., 2012; Wise u.a., 2012; Agertz u. a., 2013; Benincasa u. a., 2013; Booth u. a., 2013; Dobbs u. Pringle, 2013; Hopkins u. a., 2013; Munshi u.a., 2013; Renaud u. a., 2013; Stinson u. a., 2013, und viele mehr). Deshalb sind zum Beispiel die in Simulationen mit MIST ermittelten Sternentstehungsrelationen (Kennicutt-Schmidt-Relationen, Schmidt (1959); Kennicutt (1998)) keine direkte Folge der in das Modell eingeflossenen 'ad hoc'-Annahmen, sondern ein echtes Ergebnis der Simulation mit MIST. Resultate wie diese Relationen oder galaktische Sternentstehungsraten sind eine Konsequenz verschiedener Selbstregulationsmechanismen, die sich aus MIST sowie seiner Wechselwirkung mit Hydrodynamik und SGS-Modell in den Simulationen ergeben. Indem sich innerhalb der Simulationen gleichzeitig mehrere beobachtete Eigenschaften von Sternentstehung, Sternentstehungsregionen und sternbildenden Spiralgalaxien statistisch reproduzieren ließen, erwies sich MIST als erfolgreiches Modell. Die Struktur von MIST erlaubt es, mit relativ geringem Aufwand zusätzliche, bislang nicht berücksichtigte Prozesse einzubinden, die innerhalb des ISM wirken. Dem im Rahmen meiner Arbeit geschaffenen Modell stehen zukünftig viele interessante Anwendungsmöglichkeiten offen von der idealisierten Zwerggalaxie bis zur kosmologischen 'zoom-in'-Simulation der Entwicklung einer Galaxie. 
Wie die Abbildung auf der Titelseite eindrucksvoll zeigt, sehen die in Simulationen entwickelten idealisierten, isolierten Scheibengalaxien außerdem gut aus - dem Gesamteindruck nach echten Galaxien nicht gänzlich unähnlich (wenn man die eingezeichneten Konturen außer Acht lässt). In diesem Sinne:

\section{Sie ist ein Modell, und sie sieht gut aus.}

-Kraftwerk

Die vorliegende Dissertation gliedert sich in insgesamt sieben Kapitel.

Dieser Einleitung folgt eine kurze Einführung in die Strömungsmechanik (Kapitel 2), in der zunächst die hydrodynamischen Grundgleichungen hergeleitet werden, um danach Schall- und Stoßwellen sowie Turbulenz zu thematisieren.

Kapitel 3 führt die Begriffe Spiralgalaxie und interstellares Medium astrophysikalisch ein und gibt einen groben Überblick zum Sternentstehungsprozess.

Das neu entwickelte semi-analytische Modell des interstellaren Mediums ist Thema des Kapitels 4. Dessen innovativste Eigenschaft ist die Berücksichtigung der Turbulenzstärke als dynamische Größe innerhalb des Modells, die tief greifenden Einfluss auf sein Verhalten nimmt. Neben dem grundlegenden Konzept und den Entwicklungsgleichungen werden einige der wichtigsten Aspekte seiner Ein-ZonenGleichgewichtslösungen beleuchtet.

Im Anschluss werden die bei der Durchführung der LES von Galaxien genutzten numerischen Methoden in Kapitel 5 erläutert. Insbesondere wird auf das numerische Modell MIST eingegangen, das eine Weiterentwicklung des semi-analytischen Modells für die Verwendung in LES darstellt. Nachfolgend wird einer der durchgeführten Simulationsläufe von einer isolierten Scheibengalaxie inklusive seiner wichtigsten Resultate besprochen.

Während sich die vorigen zwei Kapitel 4 und 5 auf die bereits veröffentlichten Journalartikeln Braun u. Schmidt (2012) und Braun u. a. (2014) (siehe Anhang A.1 und A.2) beziehen, wird in Kapitel 6 eine bislang unveröffentlichte Studie präsentiert. Sie beschäftigt sich mit den Auswirkungen von verschiedenen Ansätzen zur Abschätzung von Sternentstehungsraten innerhalb molekularen Gases.

Den Abschluss (Kapitel 7) bildet eine übergreifende Zusammenfassung einschließlich eines Ausblicks auf mögliche künftige Anwendungen und Erweiterungen von MIST. 


\section{Kapitel 2}

\section{Strömungslehre}

Die Hydrodynamik, oder Fluiddynamik ${ }^{1}$, beschäftigt sich mit der statistischen, makroskopischen Beschreibung von Vielteilchensystemen. Hierbei ist die Anzahl $N$ der Teilchen sehr groß - so groß, dass eine mikroskopische Beschreibung aller beteiligten Teilchen und ihrer individuellen Freiheitsgrade schlicht unmöglich ist, was sowohl den Rechenaufwand als auch die Kenntnis der Anfangsbedingungen betrifft. Außerdem ist man im Rahmen hydrodynamischer Fragestellungen meist weder auf eine detaillierte, mikroskopische Beschreibung des Systems angewiesen, noch ist die genaue Kenntnis der mikroskopisch ablaufenden Prozesse, wie etwa individuellen Stößen zwischen Teilchen, von Interesse, solange sich diese durch statistische Beschreibungen ausreichend gut modellieren lassen, um ihre makroskopisch sichtbaren Auswirkungen abzubilden.

$\mathrm{Zu}$ diesem Zweck betrachtet man statt eines einzigen physikalischen Systems eine Vielzahl gleichartiger Systeme, die mikroskopisch betrachtet unterschiedliche Zustände aufweisen mögen, makroskopisch aber voneinander ununterscheidbar sind. Diese Gruppe von Systemen bildet das sogenannte Gibbs-Ensemble.

\subsection{Grundgleichungen der Hydrodynamik}

\subsubsection{Boltzmanngleichung}

Der makroskopische Zustand des Systems zum Zeitpunkt $t$ lässt sich durch die auf dem $N \times 6$-dimensionalen ${ }^{2}$ Phasenraum $\Gamma$ lebende Phasenraumverteilungsfunktion

\footnotetext{
${ }^{1}$ Der Begriff Hydrodynamik suggeriert etymologisch einen engen Zusammenhang mit Wasser. Üblicherweise wird er aber weiter gefasst, sodass er für alle möglichen (nicht elektrisch leitfähigen) klassischen, strömungsfähigen Medien gilt. Die Magnetohydrodynamik ist eine Erweiterung der reinen Hydrodynamik für elektrisch leitfähige Fluide. Als Beispiel für Lehrbücher über Fluiddynamik seien hier Clarke u. Carswell (2014) und Schade u. a. (2014) genannt.

${ }^{2}$ In Folgenden nehme ich an, dass jedes Teilchen genau drei räumliche Freiheitsgrade besitzt, aber keinerlei innere.
} 
$F\left(\mathbf{x}_{1}, \ldots, \mathbf{x}_{i}, \ldots, \mathbf{x}_{N}, \mathbf{p}_{1}, \ldots, \mathbf{p}_{i}, \ldots, \mathbf{p}_{N}, t\right)$ beschreiben, die wiedergibt, mit welcher Wahrscheinlichkeit sich das System in welchem mikroskopischen Zustand im zugeordneten Ensemble befindet. Wenn alle Teilchen gleichartig identisch sowie wechselwirkungsfrei sind, so sind auch ihre individuellen Phasenraumverteilungsfunktionen $f_{i}\left(\mathbf{x}_{i}, \mathbf{p}_{i}, t\right)$ identisch, wobei $\mathbf{x}_{i}$ den Orts- und $\mathbf{p}_{i}$ den Impulsvektor des $i$-ten Teilchens in dem von seinen individuellen Freiheitsgraden aufgespannten Phasenraum $\mu_{i}$ bezeichnet. Unter diesen Umständen gilt

$$
F\left(\mathbf{x}_{1}, \ldots, \mathbf{x}_{N}, \mathbf{p}_{1}, \ldots, \mathbf{p}_{N}, t\right)=\prod_{i=1}^{N} f_{i}\left(\mathbf{x}_{i}, \mathbf{p}_{i}, t\right)=[f(\mathbf{x}, \mathbf{p}, t)]^{N}
$$

wobei $f(\mathbf{x}, \mathbf{p}, t)$ die auf dem 6-dimensionalen Phasenraum $\mu$ definierte EinteilchenPhasenraumverteilung ist. Da der Satz von Liouville gilt, der besagt, dass das Phasenraumvolumen $\mathrm{d} t \Omega_{\mu}$ des Ensembles unter Zeitentwicklung konstant ist, kann folgende Zeitentwicklungsgleichung für $f(\mathbf{x}, \mathbf{p}, t)$ abgeleitet werden

$$
\frac{\mathrm{d} f}{\mathrm{~d} t}=\frac{\partial f}{\partial t}+\sum_{j=1}^{3}\left(\frac{\partial f}{\partial x_{j}} \frac{\mathrm{d} x_{j}}{\mathrm{~d} t}+\frac{\partial f}{\partial p_{j}} \frac{\mathrm{d} f}{\mathrm{~d} t}\right)=0
$$

die auch als Vlasov-Gleichung bekannt ist. Lässt man nun kurzzeitige, elastische Stöße zwischen den ansonsten wechselwirkungsfreien Teilchen zu, bei denen zwei Teilchen vermittelt durch kurzreichweitige, geschwindigkeitsunabhängige Kräfte interagieren, erhält man die Boltzmann-Gleichung

$$
\frac{\mathrm{d} f}{\mathrm{~d} t}=\frac{\partial f}{\partial t}+\nabla_{\mathbf{x}} f \frac{\mathrm{d} \mathbf{x}}{\mathrm{d} t}+\nabla_{\mathbf{u}} f \frac{\mathrm{d} \mathbf{u}}{\mathrm{d} t}=\left.\frac{\partial f}{\partial t}\right|_{\mathrm{C}},
$$

wobei

$$
\nabla_{\mathbf{x}} \equiv\left(\frac{\partial}{\partial x_{1}}, \frac{\partial}{\partial x_{2}}, \frac{\partial}{\partial x_{3}}\right) \text { und } \nabla_{\mathbf{u}} \equiv\left(\frac{\partial}{\partial u_{1}}, \frac{\partial}{\partial u_{2}}, \frac{\partial}{\partial u_{3}}\right),
$$

mit der Einteilchen-Geschwindigkeit $\mathbf{u}$. Der als Störgröße auftauchende Stoßterm $\left.(\partial f / \partial t)\right|_{\mathrm{C}}$ beschreibt auf statistische Weise, wie sich $f(\mathbf{x}, \mathbf{p}, t)$ infolge der Teilchenstöße zeitlich verändert und beinhaltet irreversible Prozesse die makroskopisch beispielsweise als Diffusion oder viskose Reibung in Erscheinung treten. Die Näherung, Stöße in dieser Weise effektiv statistisch zu behandeln, ist nur dann gültig, wenn einerseits die freie Weglänge, die ein Teilchen zwischen zwei Stößen zurücklegt, lang ist im Vergleich zu dem Wechselwirkungsradius eines Teilchens, aber andererseits diese freie Weglänge kurz ist relativ zu der Skala, auf der man das System makroskopisch betrachten möchte. Die zweite Forderung entspricht der nach häufigen Stößen, die dafür sorgen, dass sich das System überall - makroskopisch gesehen - im lokalen thermischen Gleichgewicht (LTE; local thermal equilibrium) befindet. In Gleichung (2.3) kann man $\mathrm{d} \mathbf{x} / \mathrm{d} t$ ohne Weiteres mit der Geschwindigkeit $\mathbf{u}=\mathrm{d} \mathbf{x} / \mathrm{d} t$ identifizieren, und $\mathrm{d} \mathbf{u} / \mathrm{d} t$ mit der Auswirkung eines externen mechanischen Kraftfelds, 
welches nur auf makroskopischen Skalen variieren sollte, da sonst der Stoßterm komplizierter würde. Dies ist die Schnittstelle, an der später langreichweitige, aber im Vergleich mit den Stoßkräften schwache Wechselwirkungen wie die Gravitation als $\mathrm{d} \mathbf{u} / \mathrm{d} t=-\nabla_{\mathbf{x}} \Phi$ eingebunden werden können, wobei $\Phi$ hier das Gravitationspotential ist.

\subsubsection{Momentenbildung}

Makroskopische Variablen des Vielteilchensystems, das dann als System eines strömenden, kontinuierlichen Mediums - eines Fluids - aufgefasst werden kann, erhält man durch die Bildung von Momenten der Phasenraumdichte oder Phasenraumbesetzungsfunktion $f$, die normiert ist durch

$$
\int_{\mu} f(\mathbf{x}, \mathbf{p}, t) \mathrm{d} \Omega_{\mu}=N .
$$

Dies geschieht durch Multiplikation mit den Größen

$$
\Xi_{(\alpha)} \equiv m \mathbf{u}^{\alpha} \quad \text { mit } \alpha=0,1,2, \ldots
$$

und Integration über den Impulsteil des $\mu$-Phasenraums, wobei $m$ die Masse eines Teilchens und $\mathbf{u}$ eine Einteilchen-Geschwindigkeit ist. Die drei niedrigsten Momente sind hier von besonderem Interesse, da sie eine anschauliche physikalische Bedeutung besitzen. Sie sind nämlich mit den Dichten der klassischen Erhaltungsgrößen Masse, Impuls und Energie, also $\rho, \rho \overline{\mathbf{u}}, \rho E$, identifizierbar:

$$
\begin{aligned}
\rho(\mathbf{x}, t) & =\int m f(\mathbf{x}, \mathbf{p}, t) \mathrm{d} \mathbf{p} \\
\rho \overline{\mathbf{u}}(\mathbf{x}, t) & =\int m \mathbf{u} f(\mathbf{x}, \mathbf{p}, t) \mathrm{d} \mathbf{p} \\
\rho E(\mathbf{x}, t) & =\int \frac{m}{2}|\mathbf{u}|^{2} f(\mathbf{x}, \mathbf{p}, t) \mathrm{d} \mathbf{p} .
\end{aligned}
$$

Die gesamte kinetische Energiedichte $\rho E$ und die makroskopisch sichtbare kinetische Energiedichte $\rho|\overline{\mathbf{u}}|^{2} / 2$ sind nicht gleich, da bei der Impulsraumintegration effektiv eine Mittelung der quadrierten Geschwindigkeit $|\mathbf{u}|^{2}$ beziehungsweise der Geschwindigkeit $\mathbf{u}$ selbst vorgenommen wurde, sodass $\rho E \geq \rho|\overline{\mathbf{u}}|^{2} / 2$ gilt. Die Differenz der spezifischen Energien $E-|\overline{\mathbf{u}}|^{2} / 2$ wird als spezifische thermische Energie $e$ definiert. Dies entspricht einer Aufspaltung der Geschwindigkeit $\mathbf{u}=\overline{\mathbf{u}}+\widetilde{\mathbf{u}}$ in eine mittlere makroskopisch erfahrbare Geschwindigkeit $\overline{\mathbf{u}}$ (lokale Strömungsgeschwindigkeit) und einen mikroskopisch fluktuierenden Geschwindigkeitsanteil $\widetilde{\mathbf{u}}$.

Allgemein ist das $\alpha$-te Moment $\left\langle\Xi_{(\alpha)}\right\rangle$ der auf $\mu$-Phasenraum lebenden Funktion $B(\mathbf{x}, \mathbf{p}, t)$ definiert als

$$
\left\langle\Xi_{(\alpha)}\right\rangle=\frac{1}{n} \int \Xi_{(\alpha)} B(\mathbf{x}, \mathbf{p}, t) \mathrm{d} \mathbf{p}
$$


wobei $n$ die lokale Teilchendichte ist

$$
n(\mathbf{x}, t)=\int f(\mathbf{x}, \mathbf{p}, t) \mathrm{d} \mathbf{p} .
$$

Bildet man Momente der Boltzmanngleichung (2.3) so erhält man für das $\alpha$-te Moment

$$
\begin{aligned}
\frac{\partial}{\partial t}\left\langle n \Xi_{(\alpha)}\right\rangle+\nabla_{\mathbf{x}}\left\langle n \Xi_{(\alpha)} \mathbf{u}\right\rangle & -\left(\nabla_{\mathbf{x}} \Phi\right) \cdot\left\langle n \nabla_{\mathbf{u}} \Xi_{(\alpha)}\right\rangle \\
= & \left.\int\left\langle\Xi_{(\alpha)}\right\rangle \frac{\partial f}{\partial t}\right|_{\mathrm{C}} \mathrm{d} \mathbf{p} .
\end{aligned}
$$

Als Maxwell-Boltzmann-Transportgleichungen (MBT) bezeichnet man diese Gleichungen für die zeitliche Entwicklung der Momente von $f$. Sie bilden eine hierarchische Folge von Gleichungen, die jeweils abgesehen vom $\alpha$-ten Moment von $f$ auch vom nächst höheren $(\alpha+1)$-ten Moment abhängen. Dies wird klar, wenn man bei der Betrachtung des zweiten Terms auf der linken Seite von Gleichung (2.9) beachtet, dass $\Xi_{(\alpha+1)}=\Xi_{(\alpha)} \mathbf{u}$ gilt. Zur Lösung des Gleichungssystems, welches die MBT darstellen, ist daher eine unabhängige Schließungsbedingung erforderlich, die das $(\alpha+1)$-te Moment als Funktion niedrigerer Momente darstellt, um nach dem $\alpha$-ten Glied der Folge abbrechen zu können.

Die rechte Seite von Gleichung (2.9) verschwindet für Größen, die bei Teilchenkollisionen insgesamt erhalten bleiben. Dies ist im Rahmen klassischer Mechanik für die fünf Größen Masse, Impuls (für jede der drei Raumrichtungen einzeln) und Energie der Fall. Die makroskopischen Auswirkungen der Teilchenstöße müssen nun also innerhalb der Schließungsrelationen berücksichtigt werden.

\subsubsection{Navier-Stokes-Gleichungen}

Die üblichen hydrodynamischen Gleichungen erhält man, indem die Momente von $f$ in die ersten drei der MBT eingesetzt werden, wobei einige Näherungen als Schließungen verwendet werden müssen.

Die Zeitentwicklung der Massendichte $\left\langle n \Xi_{(0)}\right\rangle=\rho$ ergibt sich aus dem $(\alpha=0)$-ten Moment der Boltzmanngleichung

$$
\frac{\partial \rho}{\partial t}+\nabla_{\mathbf{x}} \cdot(\rho \overline{\mathbf{u}})=0
$$

da einerseits sowohl die Masse als auch die Teilchenzahl konstant sind. Die Gleichung (2.10) ist auch als Kontinuitätsgleichung bekannt.

Die Erhaltungsgleichung für den makroskopischen Impuls erhält man aus dem $(\alpha=1)$ Moment der Boltzmanngleichung mit $\left\langle n \Xi_{(1)}\right\rangle=\rho \overline{\mathbf{u}} \mathrm{zu}$

$$
\frac{\partial}{\partial t}(\rho \overline{\mathbf{u}})+\nabla_{\mathbf{x}} \cdot[\rho\langle\mathbf{u} \otimes \mathbf{u}\rangle]-\rho \nabla_{\mathbf{x}} \Phi=0,
$$


wobei $\mathbf{u} \otimes \mathbf{u}$ die symmetrische Dyade der Geschwindigkeit $\mathbf{u}$ ist, deren Elemente $u_{i} u_{k}$ (mit $i, k \in[1,2,3])$ sind. Da sich $\mathbf{u}=\overline{\mathbf{u}}+\widetilde{\mathbf{u}}$ aufspalten lässt gilt

$$
\langle\mathbf{u} \otimes \mathbf{u}\rangle=\langle\overline{\mathbf{u}} \otimes \overline{\mathbf{u}}\rangle+\langle\widetilde{\mathbf{u}} \otimes \widetilde{\mathbf{u}}\rangle
$$

da $\overline{\mathbf{u}}=\langle\overline{\mathbf{u}}\rangle$ bereits der Mittelwert ist und $\widetilde{\mathbf{u}}$ im Mittel verschwindet $\langle\widetilde{\mathbf{u}}\rangle=0$. Eingesetzt in Gleichung (2.11) erhält man

$$
\frac{\partial}{\partial t}(\rho \overline{\mathbf{u}})+\nabla_{\mathbf{x}} \cdot[\rho\langle\overline{\mathbf{u}} \otimes \overline{\mathbf{u}}\rangle]+\nabla_{\mathbf{x}} \boldsymbol{\Pi}-\rho \nabla_{\mathbf{x}} \Phi=0,
$$

mit dem Druck- oder Spannungstensor $\Pi$, der meist komponentenweise in der Form

$$
\Pi_{i j}=P \delta_{i, j}-\pi_{i j}
$$

geschrieben wird, mit dem Kroneckersymbol $\delta_{i, j}=0$, falls $i \neq j$, und $\delta_{i, j}=1$ anderenfalls. Die Spur des Spannungstensors wird hier als der isotrope Gasdruck $P$ interpretiert. An dieser Stelle verwendet man die aus der Thermodynamik bekannte thermische Zustandsgleichung als Schließungsterm

$$
P \equiv(\gamma-1) \rho e
$$

mit dem Polytropenexponenten $\gamma=5 / 3 .^{3}$ Der Viskositätstensor $\boldsymbol{\pi}$ ist also der spurfreie Teil des Spannungstensors, der üblicherweise wie folgend unter Verwendung empirischer, spezifischer Materialeigenschaften eines Fluids, nämlich der kinematischen Scherungsviskositätskonstante $\nu$ und der Dehnungsviskositätskonstante $\zeta$, genähert wird

$$
\pi_{i j}=\rho P-\rho\langle\widetilde{\mathbf{u}} \otimes \widetilde{\mathbf{u}}\rangle=\nu \rho\left(\frac{\partial \bar{u}_{i}}{\partial x_{j}}+\frac{\partial \bar{u}_{j}}{\partial x_{i}}-\frac{2}{3} \delta_{i, k} \nabla_{\mathbf{x}} \cdot \overline{\mathbf{u}}\right)-\zeta \rho \delta_{i, j} \nabla_{\mathbf{x}} \cdot \overline{\mathbf{u}},
$$

der gewissermaßen eine Diffusion des makroskopischen Impulses beschreibt.

Die hydrodynamische Gleichung für die Gesamtenergiedichte ergibt sich aus dem $(\alpha=2)$ Moment der Boltzmanngleichung mit $\left\langle n \Xi_{(2)}\right\rangle=2 \rho E$, wobei bereits die oben eingeführten Größen $P$ und $\boldsymbol{\pi}$ eingesetzt wurden

$$
\begin{aligned}
\frac{\partial}{\partial t}(\rho E) & +\nabla_{\mathbf{x}} \cdot[(\rho E+P) \overline{\mathbf{u}}] \\
& +\nabla_{\mathbf{x}} \cdot\left(\rho\left\langle\frac{\widetilde{\mathbf{u}}}{2}|\widetilde{\mathbf{u}}|^{2}\right\rangle\right)-\nabla_{\mathbf{x}} \cdot(\boldsymbol{\pi} \overline{\mathbf{u}})-\rho \overline{\mathbf{u}} \nabla_{\mathbf{x}} \Phi=0 .
\end{aligned}
$$

Hier taucht der Term

$$
\nabla_{\mathbf{x}} \mathbf{h} \equiv \nabla_{\mathbf{x}} \cdot\left(\rho\left\langle\frac{\widetilde{\mathbf{u}}}{2}|\widetilde{\mathbf{u}}|^{2}\right\rangle\right)
$$

\footnotetext{
${ }^{3} \gamma=c_{\mathrm{P}} / c_{\mathrm{V}}=5 / 3$ ist das Verhältnis der beiden spezifischen Wärmekapazitäten $c_{\mathrm{P}}$ für isobare Zustandsänderungen und $c_{\mathrm{V}}$ für isochore.
} 
auf, der den Transport thermischer Energie $\rho e \propto \rho\left\langle|\widetilde{\mathbf{u}}|^{2}\right\rangle$ durch mikroskopische Geschwindigkeitsfluktuationen $\widetilde{\mathbf{u}}$ beschreibt. Dieser Vorgang wird als Wärmeleitung verstanden und mittels eines empirischen Diffusionsansatzes genähert

$$
\mathbf{h}=-\kappa_{e} \nabla_{\mathbf{x}} e,
$$

wobei $\kappa_{e}$ der materialspezifische Wärmeleitungskoeffizient ist.

Die drei in diesem Absatz erklärten Gleichungen bilden den als Navier-StokesGleichungen bekannten Satz partieller Differentialgleichungen

$$
\begin{gathered}
\frac{\partial}{\partial t} \rho+\nabla_{\mathbf{x}} \cdot(\rho \overline{\mathbf{u}})=0, \\
\frac{\partial}{\partial t}(\rho \overline{\mathbf{u}})+\rho \nabla_{\mathbf{x}} \cdot(\overline{\mathbf{u}} \otimes \overline{\mathbf{u}})+\nabla_{\mathbf{x}} P-\nabla_{\mathbf{x}} \cdot \boldsymbol{\pi}-\rho \nabla_{\mathbf{x}} \Phi=0 \\
\frac{\partial}{\partial t}(\rho E)+\nabla_{\mathbf{x}} \cdot[(\rho E+P) \overline{\mathbf{u}}]-\nabla_{\mathbf{x}} \mathbf{h}-\nabla_{\mathbf{x}} \cdot(\boldsymbol{\pi} \overline{\mathbf{u}})-\rho \overline{\mathbf{u}} \nabla_{\mathbf{x}} \Phi=0 .
\end{gathered}
$$

\subsubsection{Euler-Gleichungen}

Vernachlässigt man alle Effekte zweiter Ordnung, indem explizit $\nu=0, \zeta=0$ und $\kappa_{e}=0$ werden, so erhält man die für ein ideales Fluid, in dem nur rein adiabatische Zustandsänderungen möglich sind, geltenden Euler-Gleichungen

$$
\begin{gathered}
\frac{\partial}{\partial t} \rho+\nabla_{\mathbf{x}} \cdot(\rho \overline{\mathbf{u}})=0 \\
\frac{\partial}{\partial t}(\rho \overline{\mathbf{u}})+\rho \nabla_{\mathbf{x}} \cdot(\overline{\mathbf{u}} \otimes \overline{\mathbf{u}})+\nabla_{\mathbf{x}} P-\rho \nabla_{\mathbf{x}} \Phi=0 \\
\frac{\partial}{\partial t}(\rho E)+\nabla_{\mathbf{x}} \cdot[(\rho E+P) \overline{\mathbf{u}}]-\rho \overline{\mathbf{u}} \nabla_{\mathbf{x}} \Phi=0 .
\end{gathered}
$$

Resultiert aus dem externen Potential keine Kraft, die auf das Fluid wirkt, wenn also $\Phi=$ const., dann ist die Entropie $\rho S$ des Gases eine Erhaltungsgröße

$$
\frac{\partial}{\partial t}(\rho S)+\nabla_{\mathbf{x}} \cdot(\rho S \overline{\mathbf{u}})=0 .
$$

Die Euler-Gleichungen werden oft als skalenfrei bezeichnet, da sich Systeme, die sich durch einfache lineare Skalierungen ineinander überführen lassen, unabhängig von ihrer tatsächlichen physikalischen Größe in Relation genau gleich verhalten. Diese Skalenfreiheit wird sowohl durch die Einführung eines dynamischen Kraftfeldes wie der Eigengravitation des Fluids sowie das Berücksichtigen einer physikalischen Viskosität $\nu>0$ gebrochen.

In der numerischen Astrophysik werden oft die Eulergleichungen für das baryonische Gas verwendet, wo die Dichte für irdische Verhältnisse verschwindend klein ist, da innere Reibung und Wärmeleitung hier äußerst ineffizient sind - zumindest auf den üblicherweise betrachteten Skalen, die abhängig von der spezifische Fragestellung von einigen Kiloparsec bis herunter zu hundertsteln eines Parsecs messen. 


\section{$2.2 \quad$ Stöße}

Als Lösungen der hydrodynamischen Gleichungen tauchen Wellen auf, die zwar Energie, aber effektiv kein Material bewegen. Am bekanntesten sind die sich als Lösung der linearisierten Euler-Gleichungen ergebenden Schallwellen. Man erhält sie durch Betrachten einer kleinen Störung auf einem homogenen Hintergrund ${ }^{4}$, Subtrahieren der Gleichungen für den homogenen Zustand und Vernachlässigen aller Terme höherer Ordnung - also solchen, in denen gestörte Größen $y_{\sim}=y-y_{\text {homogen }}$ quadratisch oder in höherer Potenz vorkommen. Die Dichtestörungen in einer Schallwelle gehorchen der Wellengleichung

$$
\frac{\partial^{2} \rho_{\sim}}{\partial t^{2}}=c_{\text {homogen }}^{2} \nabla_{\mathbf{x}} \rho_{\sim},
$$

mit der Ausbreitungsgeschwindigkeit $c_{\text {homogen, }}$ die als Schallgeschwindigkeit bezeichnet wird

$$
c_{\text {homogen }} \equiv \sqrt{\frac{\mathrm{d} P_{\text {homogen }}}{\mathrm{d} \rho_{\text {homogen }}}}=\sqrt{\gamma(\gamma-1) e_{\text {homogen }}} .
$$

Verwendet man für die Schallgeschwindigkeit statt der homogenen Zustandsvariablen die tatsächlichen lokalen Werte, so verformt sich die Welle mit der Zeit ${ }^{5}$, da

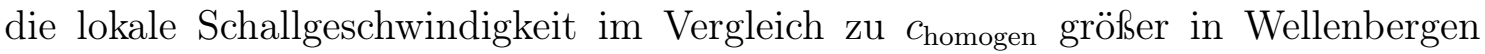
$\left(\rho>\rho_{\text {homogen }}\right)$ und kleiner in Wellentälern $\left(\rho<\rho_{\text {homogen }}\right)$ ist. Dies führt mit der Zeit zu einer Aufsteilung der Welle um die aufsteigenden Knoten $\left(\rho=\rho_{\text {homogen }}\right.$ mit $\partial \rho / \partial x_{c}>0$ in Ausbreitungsrichtung $\left.x_{c}\right)$ und einer Verflachung um die absteigenden Knoten. Diese Betrachtung bricht spätestens in dem Moment zusammen, in dem die maximale und die minimale Amplitude am aufsteigenden Knoten zusammentreffen. Es bildet sich eine Diskontinuität aus, eine sogenannte Stoßfront, die sich der Beschreibung innerhalb der linearisierten Theorie entzieht. Der Stoßfront folgt eine Verdünnungswelle, in der das Fluid wieder in den Prästoßzustand zurückgeführt wird. In Gasen mit endlicher Viskosität kommt es allerdings nicht zu solch extremen Aufsteilungen der Schallwelle, da ihre Energie in Wärme dissipiert wird, was umso effizienter wird, je weiter die Welle aufgesteilt wurde.

Stoßwellen (englisch: shock-waves) sind ein Phänomen, das sich in kompressiblen, strömenden Systemen häufig zeigt, in denen sich benachbarte Teile des Fluids relativ zueinander mit Strömungsgeschwindigkeiten bewegen, die vergleichbar mit der lokalen Schallgeschwindigkeit oder gar schneller sind. Dabei wird das Gas an der Stoßfront beim Durchlaufen derselben gleichzeitig instantan komprimiert, aufgeheizt

\footnotetext{
${ }^{4}$ Um den Hintergrund als homogen zu betrachten, reicht es aus, wenn die Variationen des Hintergrunds auf Längenskalen vergleichbar mit der Wellenlänge der Störung gegenüber der Amplitude der Störung selbst vernachlässigbar klein sind.

${ }^{5}$ Falls $\gamma>1$.
} 
und abgebremst. Im Ruhebezugssystem der Stoßfront ${ }^{6}$ strömt das Fluid mit einer Geschwindigkeit $\left|\bar{u}_{1}\right|>c_{2}$ auf die Stoßfront zu, während es sich hinter ihr mit einer Geschwindigkeit $\left|\bar{u}_{2}\right|<c_{2}$ entfernt. In diesem Bezugssystem gelten zwischen hydrodynamischen Variablen vor und nach der Diskontinuität die Rankine-HugoniotSprungbedingungen, die die Erhaltung von Masse, Impuls und Energie beim Strom über die Diskontinuität hinweg beinhalten

$$
\begin{aligned}
\rho_{1} \overline{\mathbf{u}}_{1} & =\rho_{2} \overline{\mathbf{u}}_{2} \\
\rho_{1}\left|\overline{\mathbf{u}}_{1}\right|^{2}+P_{1} & =\rho_{2}\left|\overline{\mathbf{u}}_{2}\right|^{2}+P_{2} \\
\frac{1}{2}\left|\overline{\mathbf{u}}_{1}^{2}\right|+\frac{\gamma P_{1}}{(\gamma-1) \rho_{1}} & =\frac{1}{2}\left|\overline{\mathbf{u}}_{2}^{2}\right|+\frac{\gamma P_{2}}{(\gamma-1) \rho_{2}} .
\end{aligned}
$$

Es ist offensichtlich, dass Stöße nur in kompressiblen Medien auftreten können, da beim Stoß eine nicht vernachlässigbare Verdichtung des Fluids um den Faktor $\rho_{2} / \rho_{1}=\bar{u}_{1} / \bar{u}_{2}$ geschieht. Das Kompressionsverhältnis lässt sich aus den Sprungbedingungen genauer bestimmen

$$
\frac{\rho_{2}}{\rho_{1}}=\frac{\bar{u}_{1}}{\bar{u}_{2}}=\frac{(\gamma+1) \mathcal{M}_{1}^{2}}{(\gamma+1)+(\gamma-1)\left(\mathcal{M}_{1}^{2}-1\right)} .
$$

In einem Fluid mit $\gamma=5 / 3$ ist das Kompressionsverhältnis also maximal $\rho_{2} /\left.\rho_{1}\right|_{\max }=$ 4, egal wie groß die Mach-Zahl $\mathcal{M}_{1}$ ist. Die Mach-Zahl ist definiert als dimensionsloses Verhältnis zwischen Strömungs- und Schallgeschwindigkeit

$$
\mathcal{M} \equiv \frac{u}{c}=\sqrt{\frac{u^{2}}{\gamma(\gamma-1) e}}
$$

Sind dissipative Prozesse wirksam, ist dies nicht der Fall. Im Grenzfall eines effektiv isothermen Gases mit $\gamma=1$ gilt

$$
\frac{\rho_{2}}{\rho_{1}}=\mathcal{M}_{1}^{2}
$$

Zur Lösung des effektiven Strömungsverhaltens bei Auftreten von Stößen kann die Methode der Charakteristiken benutzt werden, auf die hier nicht näher eingegangen werden soll. Diese wird auch in numerischen Verfahren genutzt, wenn die hydrodynamischen Gleichungen auf einem Euler'schen Rechengitter schrittweise mittels des sogenannten Godunov-Verfahrens integriert werden sollen. Hierbei muss nämlich in jeder Iteration für jede Gitterzellengrenze ein Riemannproblem gelöst werden. Dieses Problem besteht darin, dass der Zustand des Fluids zu einer bestimmten Zeit nach dem Entfernen einer (gedachten) Trennwand berechnet werden soll. Die Trennwand befindet sich an der Grenze zwischen zwei Teilvolumina, in denen unterschiedliche, aber zu Beginn homogene Zustände herrschen.

\footnotetext{
${ }^{6}$ Hydrodynamischen Variablen $(y)$ stromaufwärts der Stoßfront werden im Folgenden mit Subskript '1' gekennzeichnet $\left(y_{1}\right)$ solche stromabwärts mit Subskript '2' $\left(y_{2}\right)$.
} 


\subsection{Turbulenz}

Turbulenz ist ein emergentes Phänomen in fluiden Medien wie Gasen und Flüssigkeiten ${ }^{7}$. Es kursieren viele unterschiedliche Definitionen der Turbulenz, die enger oder auch weiter gefasst sind. Im Allgemeinen zeichnet sie sich durch ungeordnete, zeitlich und räumlich fluktuierende Bewegungen des Fluids aus, die einen weiten dynamischen Bereich innerhalb des strömenden Systems erfassen können. Sie tritt auf, wenn die innere Reibung des strömenden Mediums gegenüber den dynamischen Kräften vernachlässigbar klein ist.

Im Alltag begegnet man häufig turbulenten Phänomenen. Insbesondere turbulente Konvektion ist hier zu erwähnen. So geht man zum Beispiel davon aus, dass das Erdmagnetfeld ein Resultat turbulenter Konvektionsbewegungen im flüssigen Erdkern ist, die durch Radioaktivität und Kristallisation produzierte sowie die aus der Entstehungszeit der Erde verbliebene Wärme nach außen hin transportieren. Aber nicht nur in nach menschlichen Maßstäben großen Systemen wie beim Wetter oder bei Meeresströmungen findet sich Turbulenz. Sie ist auch im kleinen Maßstab anzutreffen, etwa in Luftströmungen, wie sie oberhalb einer brennenden Kerze oder Zigarette zu beobachten sind. Auch das Rauschen, das man in der Nähe von Gasleitungen, beim Ein- und Ausatmen, oder beim Abhören des Herzens wahrnehmen kann, ist ein Strömungsgeräusch, dessen Auftreten sich durch turbulente Bewegungen erklären lässt. Alle hier angesprochenen Strömungen finden im Unterschallbereich statt, was bedeutet, dass diese als weitestgehend inkompressibel aufgefasst werden können ${ }^{8}$. Charakteristisch für inkompressible, turbulente Strömungen ist das Strömungsmuster des Wirbels. Es bilden sich oft komplexe, aus vielen einzelnen Wirbeln zusammengesetzte Strukturen, die man von Rauch oder Wolken kennt, wie sie beispielsweise im Bild auf der Umschlagsrückseite zu sehen sind. Daher wird das Auftreten von Wirbeln häufig mit Turbulenz gleichgesetzt. In Überschallströmungen jedoch tritt die Bedeutung der Wirbel gegebenenfalls hinter die der in solchen Strömungen möglichen Stoßwellen zurück.

Eine häufige Eigenschaft von turbulenten Systemen ist die sogenannte Intermittenz. Intermittenz ist dann gegeben, wenn ein nicht-lineares System sich meist regulär, oder 'linear' verhält, wobei 'meist' hier sowohl im zeitlichen als auch räumlichen Sinne verstanden werden kann. Lokal und/oder kurzzeitig zeigt es aber bisweilen völlig anderes, chaotisches Verhalten. Beispiele für solches Verhalten sind Umpolungen des Erdmagnetfeldes, der sogenannte Schmetterlingseffekt, Sonnenstürme, oder

\footnotetext{
${ }^{7}$ Lehrbücher zum Thema sind beispielsweise Frisch (1995) und Pope (2000).

${ }^{8}$ Die Abweichung eines realen Systems von idealer Inkompressibilität kann die Entstehung von Schallwellen bedingen, die in Überlagerung das bereits erwähnte Strömungsgeräusch bilden.
} 
- etwas alltäglicher - Windböen ${ }^{9}$. Nicht zuletzt wegen der Intermittenz entzieht sich Turbulenz bis heute einer erschöpfenden, theoretischen Beschreibung, zumal nicht klar ist, ob es eine gänzlich allgemeine Beschreibung geben kann, und ob es überhaupt universelle Eigenschaften turbulenter Strömungen allgemein oder innerhalb verschiedener Klassen von Strömungen gibt.

\subsubsection{Reynolds-Zahl und turbulenter Übergang}

Schätzt man das Verhältnis der innerhalb eines strömenden Fluids wirkenden Trägheitskräfte und viskosen Kräfte zueinander ab, so erhält man die dimensionslose hydrodynamische Maßzahl, die als Reynolds-Zahl bekannt ist

$$
R e=\frac{\rho u^{2} L^{2}}{\nu \rho u L}=\frac{u L}{\nu}
$$

wobei hier $\rho$ die mittlere Dichte und $\nu$ die kinematische Viskositätskonstante ist. $u$ und $L$ bezeichnen charakteristische Geschwindigkeits- beziehungsweise Längenskalen, welche je nach betrachtetem System unterschiedlich definiert sein können. Grundsätzlich gilt; je größer Re ist, desto größer ist die Neigung der Strömung Turbulenz zu entwickeln. Kleinere Re hingegen sprechen eher für eine laminare, von viskosen Effekten bestimmte Strömung. In der technischen Anwendung ist Re relevant, da sich Strömungssysteme mit gleichen Reynolds-Zahlen gleich verhalten, wobei allerdings je nach betrachtetem System gegebenenfalls auch andere Maßzahlen - wie zum Beispiel im Falle kompressibler Strömungen die Mach-Zahl $\mathcal{M}$ - übereinstimmen müssen. Dieser Umstand ermöglicht es Systeme experimentell oder numerisch anhand von entsprechend skalierten Modellsystemen zu untersuchen, wie es experimentell beispielsweise für verkleinerte Modelle von Flugzeugen, Windrädern, Kraftfahrzeugen oder auch Schiffen im Windkanal beziehungsweise im Schlepptank praktiziert wird, um diese strömungstechnisch zu optimieren.

Der Übergang von einer laminaren zu einer turbulenten Strömung geschieht nicht bei einer bestimmten Re. Vielmehr zeigt es sich, dass es einen Übergangsbereich gibt, der sich je nach Randbedingungen bei anderen Reynolds-Zahlen vollzieht, wobei die Neigung zu turbulentem Verhalten graduell mit Re zunimmt. Gerade der turbulente Übergang ist Gegenstand vieler ungelöster Fragen, auch weil er äußerst sensibel ist gegenüber kleinen Störungen oder minimaler Veränderung von Randbedingungen, wie etwa der Oberflächenbeschaffenheit eines um- oder überströmten Objekts. So lässt sich der Strömungswiderstand einer umströmten Kugel durch kleine Dellen

\footnotetext{
${ }^{9} \mathrm{Je}$ nach dem, wie häufig ein intermittentes Phänomen innerhalb eines Systems auftritt, kann sein mittlerer Effekt im Rahmen einer statistischen Beschreibung des Systems erfasst werden. Dies hängt von den betrachteten Längen- und Zeitskalen ab. So sind Stoßwellen im ISM auf Skalen von einigen zehn Parsec ausreichend häufig, um ihre Effekte statistisch zu beschreiben. Kollisionen kalter Gaswolken mit einigen Parsec Ausdehnung sind hingegen zu selten zu erwarten.
} 
drastisch gegenüber dem einer glatten Kugel senken, da diese Oberflächentextur die Ausbildung einer turbulenten Grenzschicht begünstigt, welche wiederum eine Verkleinerung der Wirbelschleppe bewirkt. Dieses Phänomen nutzt man beim mit sogenannten 'dimples' versehenen Golfball aus. Bei der Entstehung von Turbulenz spielen verschiedene Strömungsinstabilitäten eine wichtige Rolle. In Unterschallströmungen ist in diesem Zusammenhang besonders die Kelvin-Helmholtz-Instabilität zu nennen, welche das Anwachsen anfänglich beliebig kleiner Störungen in der Scherschicht zwischen zwei Bereichen unterschiedlicher Strömungsgeschwindigkeit bewirkt.

Oft beobachtet man mit zunehmender Reynolds-Zahl verschiedene Strömungsmuster, deren Symmetrien immer höhergradig werden, bis für sehr hohe Re schließlich vollständige räumliche sowie zeitliche Rotations- und Translationsinvarianz (innerhalb des von Turbulenz erfassten Bereichs) im statistischen Sinne gegeben ist. Solche Turbulenz kann also statistisch betrachtet als homogen und isotrop aufgefasst werden. Man spricht in diesem Fall von voll entwickelter Turbulenz. Für astrophysikalische Systeme wie Gas innerhalb einer galaktischen Scheibe ist Re äußerst groß, da bei einer bisher nicht näher bestimmten/bestimmbaren, aber gewiss geringen Viskosität die typischen Abmessungen in der Größenordnung von Kiloparsec sind, und die entsprechenden Geschwindigkeiten im Bereich von $10 \ldots 10^{3} \mathrm{~km} \mathrm{~s}^{-1} \operatorname{liegen}^{10}$.

\subsubsection{Kolmogorov Theorie}

Das von Richardson Anfang der 1920er Jahre vorgeschlagene Bild für voll ausgebildete Turbulenz im Unterschallbereich geht davon aus, dass kinetische Energie einem strömungsfähigen System durch auf relativ großen Längenskalen variable Kräfte zugeführt wird, aber erst durch Bewegungen auf wesentlich kleineren Skalen durch viskose Effekte in Wärme dissipiert werden kann. Dazwischen wird die Energie von durch Instabilitäten entstehenden Wirbeln bis hinunter zur Dissipationsskala transportiert. Dabei wird die Energie von größeren Wirbeln an immer kleinere Wirbel, die sich durch parasitäre Instabilitäten (Kelvin-Helmholtz-Instabilität) in den Wirbelströmungen formen, weitergereicht. Dies ist die sogenannte turbulente Kaskade. Die drei Bereiche im Fourierraum der räumlichen Geschwindigkeitsvariationen werden als Injektions-, Inertial- beziehungsweise Dissipationsbereich bezeichnet. Basierend auf dieser Vorstellung, die in Abb. 2.1 skizzenhaft dargestellt ist, entwickelte Kolmogorov seine Turbulenztheorie in einer Reihe von Veröffentlichungen (siehe Kolmogorov, 1941a,b,c, und weitere).

Die Hypothese, auf die sich diese Theorie stützt, lautet: Die statistischen Eigenschaften eines strömenden Systems mit sehr großer Reynolds-Zahl sind auf kleinen Skalen ausschließlich und eindeutig durch die betrachtete Längenskala $\ell \propto k^{-1}$, die mitt-

\footnotetext{
${ }^{10}$ Selbst wenn das interstellare Medium die für ein Gas unrealistisch große Viskosität von Wasser $\left(\nu \sim 10^{-3} \mathrm{~m}^{2} \mathrm{~s}^{-1}\right)$ besäße, entspräche dies einer Reynolds-Zahl von $\operatorname{Re} \gg 10^{22}$.
} 
lere spezifische Dissipationsrate $\epsilon$ und die Viskosität $\nu$ bestimmt ${ }^{11}$. Dies impliziert, dass der spektrale Energiefluss von großen zu kleinen Skalen hin über den gesamten Inertialbereich hinweg - statistisch betrachtet - konstant bleibt. Dies kann damit begründet werden, dass sich die kleinen Wirbel am unteren Ende der Kaskade gemessen an den Zeitskalen, die für die Wirbel an ihrem Beginn relevant sind, schnell anpassen können, so dass diese stets im statistischen Gleichgewicht mit der Injektion sind. Folglich müssen sich gegebene Energieinjektionsrate $\Pi / \rho$ und sich ergebende Dissipationsrate $\epsilon$ die Waage halten.

Insbesondere folgt aus den unter dieser Annahme konstruierten Strukturfunktionen, auf die ich hier nicht weiter eingehen möchte, dass innerhalb des Inertialbereichs die spektrale Energiedichte $E(k)$ die Form eines Potenzgesetzes annimmt

$$
E(k) \propto \epsilon^{2 / 3} k^{-5 / 3}
$$

und dass für die erwartbaren Geschwindigkeitsfluktuationen $\tilde{u}$ auf verschiedenen Längenskalen $\ell$ und $\hat{\ell}$ die folgende Skalenrelation erfüllt ist

$$
\frac{\tilde{u}(\hat{\ell})}{\tilde{u}(\ell)} \propto\left(\frac{\hat{\ell}}{\ell}\right)^{\alpha \tilde{u}}
$$

mit $\alpha_{\tilde{u}}=1 / 3$. Als Kolmogorov-Skala bezeichnet man die durch Dimensionsanalyse gewonnene Längenskala $\ell_{\nu}$

$$
\ell_{\nu}=\left(\frac{\nu^{3}}{\epsilon}\right)^{1 / 4}
$$

Zusammen mit der zugehörigen Geschwindigkeitsskala $\tilde{u}_{\nu}=(\nu \epsilon)^{1 / 4}$ zeigt sich, dass dies die Längenskala ist, ab der viskose Kräfte die Strömung dominieren. Es gilt nämlich

$$
\operatorname{Re}\left(\ell_{\nu}\right)=\frac{\tilde{u}_{\nu} \ell_{\nu}}{\nu}=\left(\frac{\nu \epsilon \nu^{3}}{\epsilon}\right)^{1 / 4} \nu^{-1}=1 .
$$

An dieser Stelle lässt sich leicht der Unterschied zwischen zwei Klassen numerischer Simulationen von Strömungen verdeutlichen, die hydrodynamische Gleichungen iterativ auf raumfesten Euler'schen Rechengittern integrieren.

\footnotetext{
${ }^{11} \mathrm{Im}$ Falle einer unendlich großen Reynolds-Zahl fällt die Viskosität $\nu=0$ als Parameter weg. Dennoch ergibt sich im Rahmen der Kolmogorov-Theorie ein nicht verschwindender spektraler Energiefluss $\epsilon>0$. Der gemittelte Dissipationsterm $\epsilon$ bleibt im Grenzfall $\nu \rightarrow 0$ erhalten $\left(\epsilon=\lim _{\nu \rightarrow 0} \epsilon\right)$, da die Gradienten der Geschwindigkeiten im viskosen Spannungstensor $\boldsymbol{\pi}$ für $\nu \rightarrow 0$ ausreichend schnell divergieren. Innerhalb der Euler-Gleichungen hingegen, die 'per definitionem' keine Viskosität kennen, wird Energie zwar zu immer kleineren Skalen hin transportiert, aber niemals in Wärme umgewandelt, was irgendwann mit den bei der Konstruktion der Euler-Gleichungen getroffenen Annahmen unvereinbar werden muss, da die Fluidnäherung spätestens in der Nähe der freien Weglänge einzelner Fluidpartikel zusammenbricht. Dieser Nachteil wird in LES wegen der Diskretisierung von Raum und Zeit aber nie sichtbar (siehe Kapitel 5.1.2).
} 


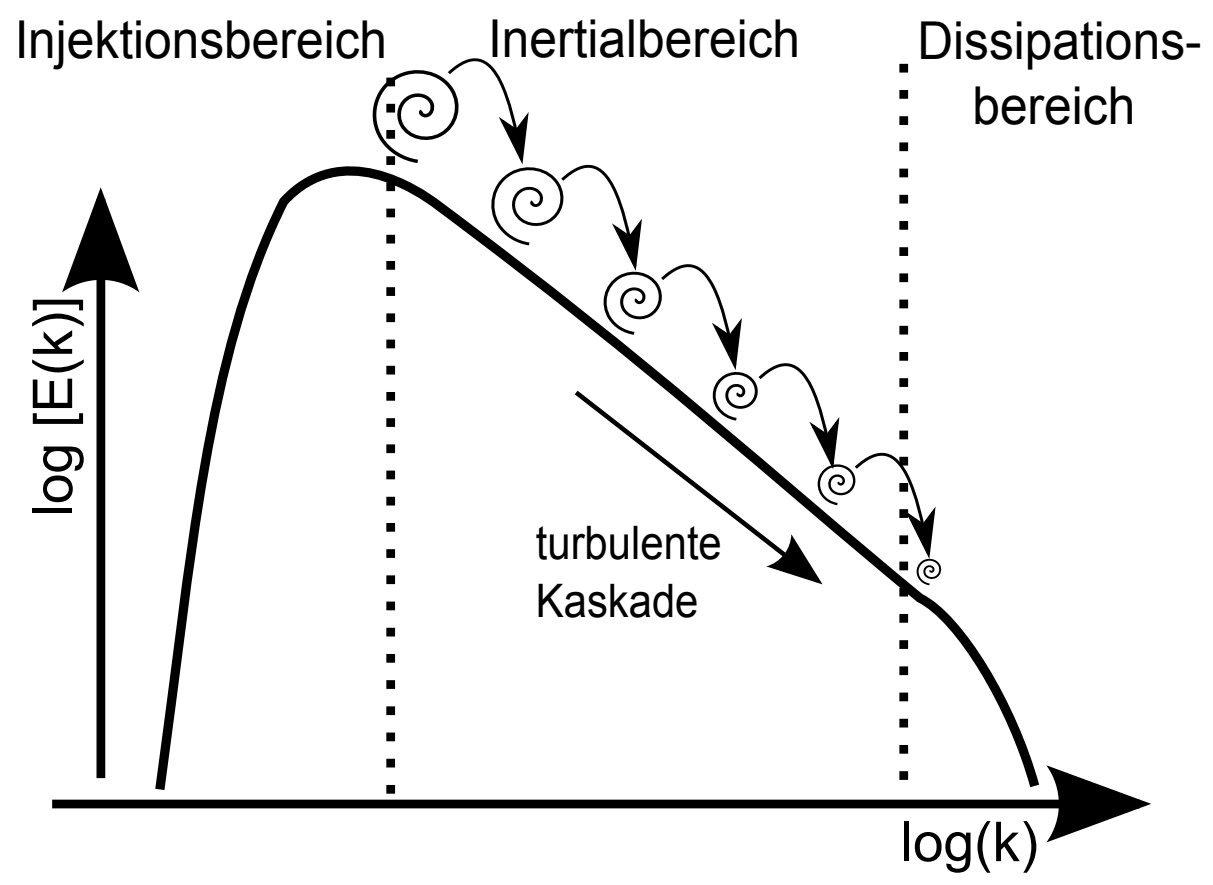

Abbildung 2.1: Skizze des turbulenten Energiespektrums $E(k)$ über der inversen Längenskala $k \propto \ell^{-1}$.

Auf der einen Seite gibt es die sogenannten direkten numerischen Simulationen (DNS). Für diese werden die vollen Navier-Stokes-Gleichungen (siehe Abschnitt 2.1.3) mit expliziter, physikalischer Viskosität gelöst. Die Anforderung hier ist, dass bei der Diskretisierung des Raumes die Kolmogorov-Skala (Gleichung 2.38) des Systems zwingend an jeder Stelle der Simulation aufgelöst werden muss, um so den gesamten relevanten Dynamikumfang des modellierten physikalischen Systems numerisch abzubilden. Leider ist dies nur für recht kleine Systeme mit relativ kleinen $R e \lesssim 10^{4}$ machbar. Man ist hier schnell durch die verfügbaren Rechnerressourcen limitiert, da die effektive Reynoldszahl einer dreidimensionalen Simulation nur langsam mit der dritten Wurzel der Anzahl numerischer Stützstellen steigt.

Auf der anderen Seite gibt es LES. Im Rahmen von LES verzichtet man bewusst darauf, den vollen Dynamikbereich eines physikalischen Systems numerisch zu modellieren. Auf dem Rechengitter werden explizit nur die reibungsfreien Euler-Gleichungen (siehe Abschnitt 2.1.4) gelöst. Hinzu kommt allerdings ein Modell, welches die Effekte der nicht mehr aufgelösten Dynamik auf die aufgelöste effektiv berücksichtigt - ein SGS-Modell. Um dies zu bewerkstelligen, können verschiedene Strategien verfolgt werden, auf die ich hier nicht näher eingehen möchte (siehe Kapitel 5.1.2). LES eignen sich besonders zur numerischen Modellierung großer Strömungssysteme mit extrem hoher Re, wenn man sich hauptsächlich für sein Verhalten auf großen Skalen interessiert. LES machen somit Simulationen von astrophysikalischen Objekten wie Gaswolken, ganzen Galaxien oder gar Simulationen kosmologischer Strukturentste- 
hung bei Berücksichtigung des baryonischen Gases erst möglich.

Die Existenz von Skalengesetzen, ähnlich denen, wie Kolmogorov sie ableitete, ist in apparativen und numerischen Experimenten für inkompressible, isotrope, homogene Turbulenz bestätigt worden, wobei die gemessenen Skalenexponenten mitunter leicht abweichen. Dies mag einerseits an experimentellen Beschränkungen liegen, kann aber andererseits auch durch die Wechselwirkung von Wirbeln verschiedener Größen verursacht werden - also die Verformung, besonders das Strecken, von kleineren Wirbeln durch größere, die in dieser Theorie nicht berücksichtigt wird. Turbulenz in Medien, die sich mit Überschallgeschwindigkeiten bewegen, die gravitieren, die elektrisch leitfähig sind, oder die chemischen und radiativen Prozessen unterworfen sind, entzieht sich der Beschreibung im Rahmen der Kolmogorov Theorie. Eine echte Universalität in Bezug auf alle vollständig turbulenten Strömungen ist nur schwer vorstellbar. Viele modernere Turbulenzmodelle verwenden die von Kolmogorov eingeführten Begriffe und Methoden für die Beschreibung der Turbulenz. So gibt es beispielsweise ein nach Burgers benanntes, auf ähnlichen Annahmen beruhendes Turbulenzmodell. Es ist konzipiert für von Stoßwellen statt von Wirbeln als charakteristischem Strömungsmuster bestimmte, reine Überschallturbulenz und schlägt eine Geschwindigkeitsskalenrelation mit einem Skalenexponenten $\alpha_{\tilde{u}}=1 / 2$ statt $\alpha_{\tilde{u}}=1 / 3$ vor. Mittels numerischer Experimente (Kritsuk u.a., 2007) aber auch analytischer Überlegungen (Galtier u. Banerjee, 2011) wurden Anstrengungen unternommen, die für inkompressible, rein hydrodynamische Turbulenz bestätigte Universalität der turbulenten Skalenbeziehungen auf Überschallturbulenz zu erweitern. Es zeigt sich, dass sich unter Verwendung der dichtegewichteten Geschwindigkeit $u_{\rho}=\rho^{1 / 3} u$ (statt der Geschwindigkeit $u$ ) in der Berechnung der Strukturfunktionen der turbulenten Strömungen ähnliche Skalenrelationen und Energiespektra $\left(E_{u_{\rho}}(k) \propto k^{-5 / 3}\right)$ in Überschall- wie im Fall von Unterschallturbulenz ergeben. Dies scheint aber nicht allgemein zu gelten, sondern hängt auch von der Art der turbulenten Moden (ob kompressiv oder solenoidal) ab (Federrath, 2013).

Im astrophysikalischen Kontext hat man es meist mit Turbulenz im Übergang zwischen Unter- und Überschallbereich zu tun. Daher erwartet man dort meist ein gemischtes $\alpha_{\tilde{u}}=1 / 3 \ldots 1 / 2$, wie sie sich auch aus Beobachtungen von Molekülwolken ableiten lassen (beispielsweise aus denen von Larson (1981)). Im Falle voll ausgebildeter, kompressibler Turbulenz in einem Gas mit isothermer Zustandsgleichung ohne weitere Wechselwirkungen nimmt die Wahrscheinlichkeitsverteilung der logarithmierten Dichten die Form einer Normalverteilung an (zum Beispiel Kritsuk u. a., 2007). Dieser Umstand wird zum Beispiel bei der Herleitung verschiedener analytischer Sternentstehungsmodelle (siehe Kapitel 6) erfolgreich genutzt, obwohl 
diese wegen ihrer Normierbarkeit vorteilhafte Form der Verteilung für selbstgravitierendes Gas eigentlich nicht anwendbar ist, wie Beobachtungen bestätigen (Hughes u. a., 2013b; Schneider u. a., 2014). 


\section{Kapitel 3}

\section{Astrophysik}

\subsection{Spiralgalaxien}

Spiralgalaxien sind der häufigste Typ von Galaxien im lokalen Universum, der überall abseits von Galaxienhaufen, aber meist in kleinen Gruppen, anzutreffen ist. Sie zeichnen sich durch sehr flache Gas- und Sternenscheiben aus, die geordnet, aber differenziell um ihr Zentrum rotieren. Die meisten besitzen um ihr Zentrum herum eine mehr oder weniger große Verdickung in der Sternenscheibe, die als 'bulge' bezeichnet wird. Außerdem lassen sich in der Gas- sowie in der Sternenscheibe meistens spiralartige Strukturen erkennen. In Anwesenheit eines Balkens sind meist zwei prominente Spiralarme zu beobachten, zwischen denen sich aber noch zahlreiche Nebenarme befinden können. Eine Galaxie solchen morphologischen Typs (SBc, also eine Balkenspiralgalaxie mit relativ kleinem 'bulge') ist die Milchstraße.

Eine Spiralgalaxie ist ein offenes, dynamisches, gravitativ gebundenes System, das im Wesentlichen aus drei massebehafteten Komponenten besteht:

- einem Halo druckfreier, dunkler Materie, der den Löwenanteil zur Gesamtmasse beiträgt und in äußeren Bereichen der galaktischen Scheibe sowie deren weiterer Umgebung maßgeblich die Rotation der sichtbaren Materie beeinflusst

- einer rotierenden Sternenscheibe, deren Existenz das gesamte Objekt erst als Galaxie qualifiziert, denn erst Sterne machen es sicht- und identifizierbar

- einer weiter ausgedehnten, ebenfalls rotierenden Gasscheibe, in der sich in besonders hoch verdichteten Bereichen Sterne bilden können.

In dieser Arbeit befasse ich mich mit der Simulation von isolierten Scheibengalaxien (englisch: isolated disk galaxies, IDG). Reale IDG in 'freier Wildbahn' sehen zum Beispiel so aus wie in Abb. 3.1 gezeigt. Die Scheibe einer IDG zeigt nur schwach ausgeprägte, unvollständig ausgebildete Spiralarme und auch sonst keine prominenten 


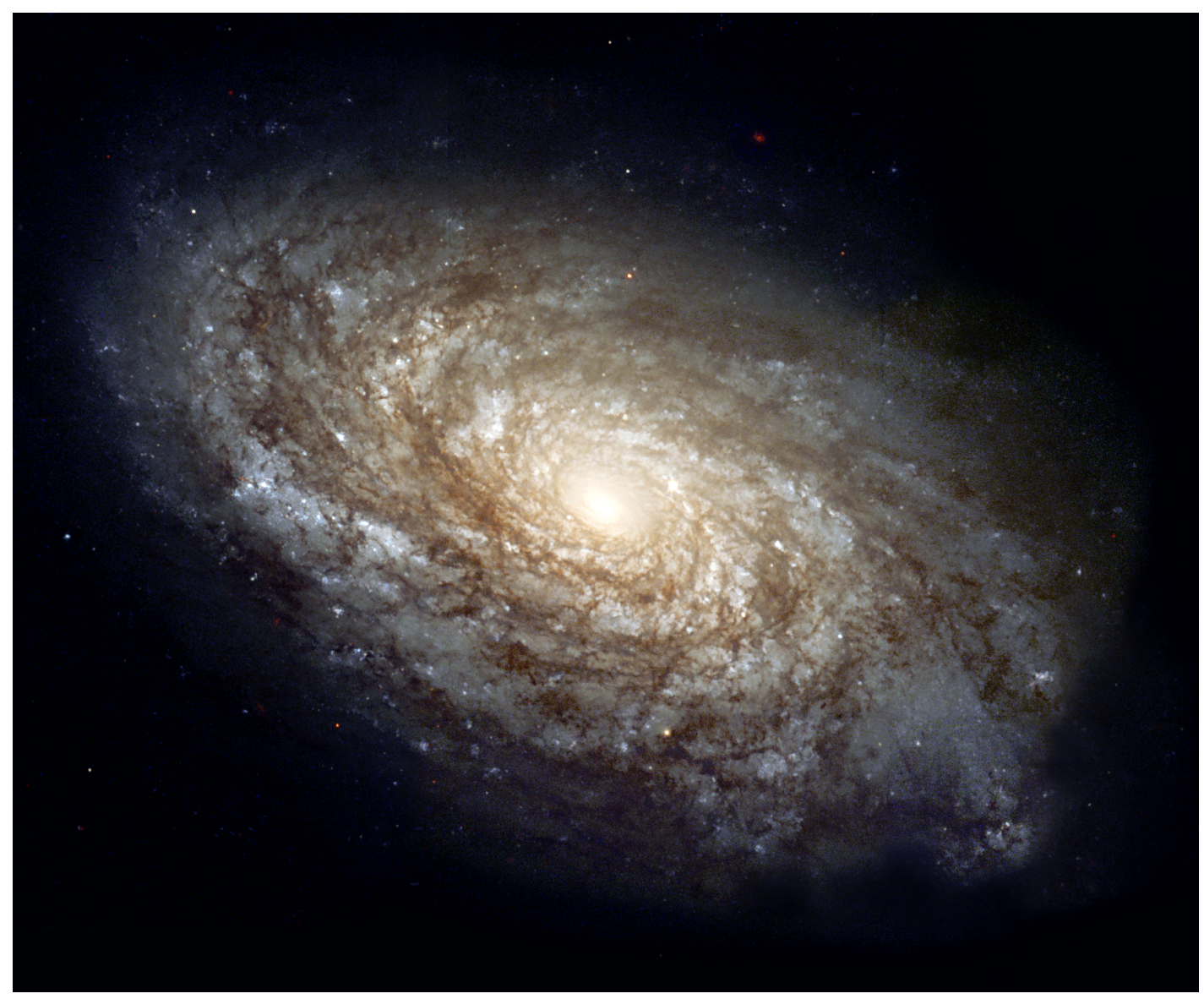

Abbildung 3.1: NGC 4414; Beispiel einer realen isolierten Scheibengalaxie vom morphologischen Typ SAc (balkenlose Spiralgalaxie mit kleinem 'bulge'), die sich im Abstand von 19,1 Mpc von der Erde befindet. Kompositbild aus Beobachtungsdaten in verschieden Wellenlängenbereichen aufgenommen vom Hubble Space Telecope (Bild: NASA, ESA, and The Hubble Heritage Team (STScI/AURA). 
Merkmale wie Balken oder Ringe, da sie weder aktuell noch in jüngerer Vergangenheit signifikanten externen Störungen durch andere Galaxien ausgesetzt war. Dazu würden auch etwaige Verschmelzungen mit anderen Galaxien vergleichbarer Masse sowie die Integration in einen Galaxienhaufen zählen. Nach Verschmelzungen von Galaxien und galaktischen Interaktionen im Allgemeinen ist Sternentstehung mit all ihren Implikationen und Konsequenzen der Prozess, der die Entwicklung einer Galaxie vorantreibt und ihre Erscheinung maßgeblich formt.

Kosmologisch betrachtet ist aber jede größere Galaxie das Produkt einer Reihe von Verschmelzungen kleinerer Objekte, welche sich in ferner Vergangenheit abgespielt haben können. Im Standardbild der kosmologischen Strukturentstehung, dessen ausführliche Beschreibung hier zu weit führen würde (siehe beispielsweise Weinberg (2008)), wurden nämlich alle größeren Strukturen, wie sie auch mit der Milchstraße vergleichbare Galaxien darstellen, in einem hierarchischen Prozess aus kleineren Elementen aufgebaut. Dies beruht darauf, dass gemäß gängigem Kosmologiemodell ${ }^{1}$ das lineare Anwachsen anfänglich kleiner, primordial bedingter Störungen $^{2}$, für kleinskalige Störungen früher in nicht-lineares Anwachsen umschlägt als für größerskalige. Bei dem nicht-linearen Anwachsen der Dichtestörungen handelt es sich um den Kollaps überdichter Bereiche unter eigener Schwerkraft, der sich entgegen dem allgemeinen Trend der kosmologischen Expansion vollzieht.

Eine reale IDG zeichnet sich in diesem Bild dadurch aus, dass ihr Aufbau im Wesentlichen bereits vor geraumer Zeit abgeschlossen war, beziehungsweise in ihrem Umfeld schlicht keine Verschmelzungs- oder Wechselwirkungsparter verblieben sind. In der Folge blieb ihr ausreichend Zeit um zu relaxieren, so dass außer einem stellaren Bulge keine morphologischen Spuren ihrer Entstehungsgeschichte mehr erkennbar sind. Ich möchte hier nicht näher auf die bekannten, durch astronomische

\footnotetext{
${ }^{1}$ Es handelt sich um das $\Lambda$ CDM-Modell. Es ist das nach bisherigem Kenntnisstand favorisierte Modell für die globale Entwicklung des Universums. Neben sichtbarer, baryonischer Materie und Strahlung als Beitrag zum Energieinhalt des Universums werden die sogenannte 'kalte dunkle Materie' (CDM; cold dark matter), die sich allein durch Gravitation bemerkbar macht, und eine ominöse 'dunkle Energie' $\Lambda$, die eine beschleunigte Expansion des Universums begünstigt, berücksichtigt. Dabei erweisen sich die 'leuchtenden' Komponenten als vergleichsweise unbedeutend, denn CDM und $\Lambda$ stellen zusammen offenbar etwa $95 \%$ der Energie.

${ }^{2}$ Diese Störungen, die sich mittlerweile im heutigen Universum zu Galaxien, Galaxienhaufen oder den als kosmischem Netz bekannten großräumigen, filament- oder schwammartigen Strukturen ausgewachsen haben, zeigen sich auch als Temperaturvariationen (in der Größenordnung von $10^{-5}$ relativ zur mittleren Temperatur) im beispielsweise vom Planck-Satelliten (siehe zum Beispiel Planck Collaboration u. a., 2013) beobachteten kosmologischen Radiowellenhintergrund (cosmologic microwave background, CMB). Der CMB gilt als Abdruck der weit weniger ausdifferenzierten Dichtestruktur des frühen Universums - gewissermaßen als in der Strahlung eingefrorene, rot verschobene Momentaufnahme der Zeit, als sich baryonisches Gas und elektromagnetische Strahlung voneinander entkoppelten.
} 
Beobachtungen abgesicherten Eigenschaften von Spiral- oder Scheibengalaxien eingehen, und verweise den Leser an einschlägige Literatur, wie beispielsweise Binney u. Merrifield (1998).

Die simulierten idealisierten IDG, die von Masse und Abmessungen her in etwa der Milchstraße entsprechen, dienen lediglich als modellhafter, prototypischer Hintergrund für die Simulation des Gases in der Scheibe, also der Sicherstellung realistischer Rahmenbedingungen zur Untersuchung des von mir entwickelten Modells für das turbulente, interstellare Medium. Sie werden innerhalb einer geschlossenen, periodischen Box simuliert, insbesondere wird keine Akkretion von Material aus dem intergalaktischen Medium modelliert. Obwohl die verwendete IDG als sogenanntes 'toy model' angesehen werden kann, liefern die durchgeführten Simulationen doch Ergebnisse, die sich gut mit denen von Beobachtungen realer Galaxien vergleichen lassen.

\subsection{Interstellares Medium}

Mit dem Begriff des interstellaren Mediums (ISM) wird alles umfasst, was innerhalb einer Galaxie das Volumen zwischen den Sternen erfüllt. Das ISM beinhaltet neben Gas in unterschiedlichsten Zuständen und Konfigurationen auch Staub, Magnetfelder, elektromagnetische Strahlung sowie kosmische Partikelstrahlung. Einen Eindruck davon, wie das ISM aussehen kann, vermittelt Abb. 3.2. Als offenes dynamisches System ist das ISM nicht nur seiner galaktischen Umgebung ausgesetzt, sondern steht auch in enger Wechselwirkung mit vom ihm umschlossenen Sternen und den stellaren Überresten, zu denen sich Sterne nach Erschöpfen ihres Brennstoffvorrates entwickeln. Innerhalb des ISM sind eine Vielzahl nicht nur extern angetriebener sondern sich auch gegenseitig beeinflussender Prozesse und Phänomene zu beobachten, die hier nicht alle detailliert aufgeführt, geschweige denn in der Komplexität ihres Zusammenwirkens erklärt werden können, zumal es dafür auch an gesicherten physikalischen Erkenntnissen fehlt. Einige chemische, radiative, magnetische, turbulente, ..., oder gravitative Prozesse mögen gut verstanden sein, doch bleiben noch viele offene Fragestellungen zu bearbeiten, zu beantworten oder gar zu entdecken. Einen Überblick zum Stand der Forschung in diesem Bereich geben unter anderem die Reviews von Molinari u. a. (2014); Kennicutt u. Evans (2012); Krumholz (2013, 2014); Schruba (2013); Tan u. a. (2013); Wilson (2013), und die Referenzen darin.

Das ISM-Gas hat typischerweise eine mittlere Teilchendichte $\sim 0,1 \mathrm{~cm}^{3}$ und besteht zu 97-100\% aus Wasserstoff und Helium. Es liegt in Zuständen vor, die sich in Bezug auf Dichte, Temperatur und chemische Zusammensetzung stark unterscheiden und räumlich separiert auftreten. Man teilt das ISM-Gas deshalb üblicherweise in 
verschiedene Phasen auf. Allerdings sind die Übergänge zwischen den einzelnen Phasen nicht abrupt, sondern kontinuierlich und fließend, da sie ständig Material und Energie mit ihrer Umgebung austauschen. Daher existiert Gas auch in beliebigen Zwischenzuständen. Die Einteilung ist dennoch sinnvoll, da sie Zustandsregimes zusammenfasst, in denen bestimmte Prozesse wirksam sind, andere aber nicht. Phasen geringerer Dichte besitzen eine höhere Temperatur und bestehen eher aus ionisiertem Gas, während diejenigen höherer Dichte kühler sind und aus neutralem atomaren oder im Fall sehr hoher Dichten sogar molekularem Gas bestehen. Wärmere Phasen sind eher diffus und füllen größere Teile des verfügbaren Volumens, kühlere neigen dazu in wärmeren Medien eingebettete Wolken oder dichte Klumpen zu bilden.

\subsubsection{Sternentstehung}

Eine besondere Bedeutung kommt der Sternentstehung zu, denn die produzierten Sterne treiben die Entwicklung einer Galaxie, aber auch einen großen Teil der Dynamik im ISM an. Leider gehört sie auch zu den komplexesten Abläufen im ISM. Sterne entstehen infolge lokalen gravitativen Kollapses von Fragmenten kalter, dichter Wolken in Gruppen als Sternhaufen oder -assoziationen. Es ist zum Beispiel noch immer Gegenstand aktueller Forschung, warum der Sternentstehungsprozess so ineffizient wie beobachtet ist, oder, warum wie viele Sterne welcher Masse gebildet werden. Zusätzlich ist auch die Frage offen, welche (auch äußeren) Faktoren den Ablauf der Sternentstehung in welcher Weise beeinflussen, oder, ob Sternentstehung überall universell gleich abläuft. Zu diesen Faktoren gehört unter anderem die Anwesenheit von Spuren schwerer Elemente ${ }^{3}$ im ISM, oder aber deren Abwesenheit. Auch das galaktische Umfeld könnte eine Rolle spielen, zum Beispiel, ob sich die Sternentstehung in einem gerade verschmelzenden Galaxienpaar vollzieht oder aber in einer isolierten Spiralgalaxie. Der grobe Ablauf des Sternentstehungsprozesses soll nachfolgend ausgehend von einer Wolke warmen, neutralen Gases mit kleinen Beimengungen von Metallen skizziert werden:

Die Wolke hat anfangs eine Teilchendichte von $\sim 0,1 \mathrm{~cm}^{3}$ und eine Temperatur um $10^{4} \mathrm{~K}$, die sich im Gleichgewicht von Heiz- und Kühlprozessen entsprechend der Gasdichte und -zusammensetzung einstellt. Innerhalb der Wolke bewegt sich das Gas turbulent mit Relativgeschwindigkeiten im Bereich der Schallgeschwindigkeit.

\footnotetext{
${ }^{3}$ Schwere Elemente werden in der Astronomie als Metalle bezeichnet. Damit gemeint sind alle chemischen Elemente, die schwerer sind als Helium, auch wenn nicht alle hier als 'Metall' bezeichneten Elemente im eigentlichen Sinne metallische Eigenschaften wie beispielsweise Natrium oder Eisen besitzen. Weiterhin wird der Massenanteil der Beimengungen von Metallen im Gas als Metallizität $Z$ bezeichnet.
} 
Die einige $10^{4}-10^{6} \mathrm{M}_{\odot}$ schwere Wolke wird durch thermischen Druck und Turbulenz marginal gegen globalen gravitativen Kollaps stabilisiert. Die radiative Abregung verschiedener stoßangeregter Zustände führt, da das Gas optisch dünn ist, zum Verlust thermischer Energie, besonders in durch Stoßwellen verdichteten Regionen. Dadurch verliert das Gas lokal an Druck und kann weiter komprimiert werden, was wiederum die Effektivität des Kühlens erhöht. Dieses Phänomen wird auch als Kühlkatastrophe bezeichnet. Grund ist eine thermische Instabilität, denn effektiv besitzt das Gas eine negative Wärmekapazität, beziehungsweise eine Zustandsgleichung mit Polytropenindex $\gamma_{\text {effektiv }}<1$. Zusätzlich steigt auch das Kompressionsvermögen der durchlaufenden Stoßfronten, da die Schallgeschwindigkeit lokal sinkt. Auf diese Weise bilden sich unterstützt durch die Eigengravitation des Gases innerhalb der Wolke verdichtete Bereiche kalten Gases, das wieder eine Gleichgewichtstemperatur von einigen 10 bis $100 \mathrm{~K}$ annehmen kann, da die relevanten Kühlprozesse wegen der geringen Gastemperatur ineffizient werden. So wird durch thermische Instabilität aus warmem neutralem Medium (WNM) kaltes neutrales Medium (CNM) gebildet. Unter diesem Vorgang entstehen lokale Druckunterschiede, die weitere Turbulenz erzeugen können. Die beiden Phasen sind alles andere als statisch, denn bei Verdünnung kann Gas leicht wieder vom CNM ins WNM übergehen.

Das kalte Gas, das klumpig im warmen eingebettet vorliegt, erreicht mittlere Teilchendichten von bis zu einigen $10 \mathrm{~cm}^{-3}$. Wasserstoff, aber auch andere Elemente können hier Moleküle bilden. Die starke Überschallturbulenz sorgt für weitere lokale Verdichtungen des Gases, die sich meist wieder aufösen, wenn sie nicht durch Staub und Molekülverbindungen in der Umgebung von externen Strahlungsquellen abgeschirmt werden. Sind sie aber abgeschirmt, so können sich lokale Verdichtungen vom Rest des Gases abkoppeln und unter ihrer eigenen Schwerkraft kollabieren, falls Turbulenz und thermischer Druck nicht ausreichen, sie zu stabilisieren oder zu zerstreuen. Man spricht in diesem Zusammenhang von (magneto-)gravo-turbulenter Fragmentation.

Durch gravitative Instabilität in Teilen des kalten Gases entstehen so Wolkenkerne, in deren Zentrum durch fortgesetzten Kollaps eine oder mehrere kompakte Gasansammlungen entstehen. Deren Inneres heizt sich auf, da die beim Kollaps frei werdende Energie nicht mehr schnell genug abgestrahlt werden kann, weil das Gas dort aufgrund der hohen Dichte opak geworden ist. Diese Objekte werden als Protosterne bezeichnet. Bei ihrer Entstehung und in ihrer weiteren Entwicklung wird ein großer Teil des an sie gebundenen Gases wieder durch Winde und Jets ausgeworfen und an das ISM zurückgegeben. Ab einem gewissen Punkt reichen Dichte und Temperatur im Inneren aus um nukleare Fusionsprozesse zu ermöglichen, die Wasserstoff 
zu Helium fusionieren. Durch vom Wasserstoffbrennen frei gesetzte Energie kann der weitere Kollaps aufgehalten werden. Die so entstandenen Gasbälle relaxieren in ein hydrostatisches Gleichgewicht und gelten fortan als Sterne.

\subsubsection{Jeans-Instabilität}

Um abzuschätzen, ob ein Bereich der Wolke gravitativ instabil ist, wird häufig das Jeans-Kriterium (Jeans, 1902) bemüht. Dieses beruht auf einer linearen Stabilitätsanalyse der Euler-Gleichungen unter Berücksichtigung der Eigengravitation des Fluids. Ausgehend von einem ruhenden, homogenen Hintergrund mit kleinen Störungen erhält man eine gegenüber Gleichung (2.27) modifizierte Wellengleichung

$$
\frac{\partial^{2} \rho_{\sim}}{\partial t^{2}}=c_{\text {homogen }}^{2} \nabla_{\mathbf{x}} \rho_{\sim}-4 \pi G \rho_{\text {homogen }},
$$

wobei $G$ die Gravitationskonstante ist. Die zugehörige Dispersionsrelation zwischen Kreisfrequenz $\omega$ und Wellenzahl $k$ einer ebenen Welle lautet folglich

$$
\omega^{2}=-c_{\text {homogen }}^{2} k^{2}+4 \pi G \rho_{\text {homogen }}
$$

Negative $\omega^{2}<0$ bedeuten, dass die zeitliche Entwicklung einer möglichen Lösung $\propto \exp (i \omega t-i k x)$ keine Schallwelle, sondern ein exponentielles Anwachsen der Amplitude der Störung ist. Störungen mit einer Wellenlänge $\lambda=2 \pi / k$ wachsen also an, wenn $\lambda>\lambda_{\mathrm{J}}$ gilt. Die kritische Wellenlänge $\lambda_{\mathrm{J}}$ - auch Jeans-Länge genannt - wird

$$
\lambda_{\mathrm{J}}=\frac{2 \pi}{k_{\mathrm{J}}}=\sqrt{\frac{\pi c_{\text {homogen }}^{2}}{G \rho_{\text {homogen }}} .}
$$

Die in einer Kugel mit Durchmesser $\lambda_{\mathrm{J}}$ eingeschlossene Masse wird als Jeans-Masse $M_{\mathrm{J}}$ bezeichnet $^{4}$

$$
M_{\mathrm{J}}=\frac{\pi^{5 / 2}}{6} \frac{c_{\text {homogen }}^{3}}{G^{3 / 2} \rho_{\text {homogen }}^{1 / 2}} .
$$

\subsubsection{Stellare Massenverteilung}

Die stellare (Anfangs-) Massenverteilung (IMF: initial mass function) $\mathrm{d} N / \mathrm{d} m$ beschreibt, wie viele Sterne $N$ einer bestimmten Masse $m$ relativ zur Gesamtmasse

\footnotetext{
${ }^{4}$ Dieses Ergebnis gleicht abgesehen vom Vorfaktor der Masse einer Bonnor-Ebert-Sphäre (Ebert, 1955; Bonnor, 1956), die aus der Stabilitätsanalyse einer homogenen Gaskugel hervorgeht. Dies führt oft zu Verwechslungen, sodass die Jeans-Masse bisweilen als ein Resultat der Stabilitätsanalyse einer Gaskugel missverstanden wird, wie zum Beispiel im deutschen Wikipedia-Artikel zum Jeans-Kriterium (http://de.wikipedia.org/wiki/Jeans-Kriterium; Stand 28. Oktober 2014).
} 
einer Sternenpopulation gebildet werden. Salpeter (1955) leitete aus Beobachtungen von Sternhaufen für Sterne mit Massen $m>M_{\odot}$ ein Potenzgesetz ab

$$
\frac{\mathrm{d} N}{\mathrm{~d} m} \propto m^{\alpha_{\mathrm{IMF}}}
$$

mit $\alpha_{\mathrm{IMF}}=-2,35$. Spätere Untersuchungen bestätigten dieses Resultat für massereiche Sterne. Außerdem zeigte sich, dass die IMF für masseärmere Sterne weniger steil ist. Beispielsweise ist die von Chabrier (2001) vorgeschlagene IMF für $m>\mathrm{M}_{\odot}$ ein Potenzgesetz mit $\alpha_{\mathrm{IMF}}=-2,3$, darunter eine log-normale Verteilung

$$
\frac{\mathrm{d} N}{\mathrm{~d} m} \propto \begin{cases}m^{-1} e^{-\frac{\log _{10}^{2}\left(\frac{m}{m_{\mathrm{Ch}}}\right)}{2 \sigma_{\mathrm{Ch}}^{2}}} & \text { für } 0.1 \mathrm{M}_{\odot}<m<1 \mathrm{M}_{\odot} \\ m^{-2.3} & \text { andernfalls, }\end{cases}
$$

wobei $\sigma_{\mathrm{Ch}}=0.69$ and $m_{\mathrm{Ch}}=0.08 \mathrm{M}_{\odot}$. Man nimmt an, dass die IMF auch variieren kann, was jedoch in Beobachtungen von Sternhaufen in der Milchstraße und ihren nächsten Nachbarn kaum sichtbar ist. Insbesondere für extrem metallarmes (oder im Extremfall primordiales) Gas wird vermutet, dass sich der Schwerpunkt der IMF zu höheren Massen hin verschiebt, also ein geringeres $\alpha_{\mathrm{IMF}} \mathrm{zu}$ erwarten ist.

\subsubsection{Kennicutt-Schmidt-Relation}

Die Kennicutt-Schmidt-Relation (KS-Relation, Schmidt (1959); Kennicutt (1998)), oft auch als KS-Gesetz bezeichnet, ist eine empirisch festgestellte Gesetzmäßigkeit, die Sternentstehungsrate $\left(\dot{\rho}_{\mathrm{s}}\right.$ oder $\left.\dot{\Sigma}_{\mathrm{s}}\right)$ und Gasdichte $\rho$ beziehungsweise -säulendichte $\Sigma$ miteinander in Form eines Potenzgesetzes verknüpft

$$
\dot{\rho}_{\mathrm{S}} \propto \rho^{\alpha_{\mathrm{KS}}}
$$

beziehungsweise

$$
\dot{\Sigma}_{\mathrm{S}} \propto \Sigma^{\alpha_{\mathrm{KS}}} .
$$

Für den Exponenten $\alpha_{\mathrm{KS}}$ schlug Schmidt (1959) einen Wert von $\alpha_{\mathrm{KS}}=2 \pm 1$ vor. Kennicutt (1998) leitete aus Beobachtungen von neutralem Gas in nahen Galaxien $\alpha_{\mathrm{KS}}=1,4 \pm 0,15 \mathrm{ab}$. Der ermittelte Wert von $\alpha_{\mathrm{KS}}$ scheint davon abzuhängen, welche räumliche Auflösung die genutzten Beobachtungsdaten besitzen, und wie die Gasdichte bestimmt wurde. Außerdem gibt es offenbar auch Unterschiede zwischen Galaxien.

Auf allzu kleinen Skalen vergleichbar mit der Ausdehnung von Sternentstehungsgebieten bricht die Korrelation scheinbar zusammen, da hier die individuellen Entwicklungsphasen der sternbildenden Gaswolken eine Rolle spielen, deren Effekt auf größeren Skalen weggemittelt würde (Onodera u. a., 2010).

Es sind derzeit drei Erklärungen für konkrete Zahlenwerte von $\alpha_{\mathrm{KS}}$ im Gespräch. 
- $\alpha_{\mathrm{KS}} \approx 1,5$ : Hierbei werden Sterne aus dem Gasreservoir $\rho$ mit einer festen Effizienz $\epsilon_{\mathrm{ff}}$ pro Freifallzeit $\tau_{\mathrm{ff}}$

$$
\tau_{\mathrm{ff}}=\sqrt{\frac{3 \pi}{32 G \rho}}
$$

gebildet. Die Sternentstehungsrate lässt sich in diesem Fall also abschätzen durch

$$
\dot{\rho}_{\mathrm{s}}=\epsilon_{\mathrm{ff}} \frac{\rho}{\tau_{\mathrm{ff}}} \propto \rho^{1,5} .
$$

Diese Relation wird in normal sternbildenden Galaxien bei Betrachtung des neutralen Gases auf Skalen von Kiloparsec gefunden, wobei die abgeleitete Effizienz im Bereich $\epsilon_{\mathrm{ff}} \lesssim 0,01$ liegt.

- $\alpha_{\mathrm{KS}} \approx 1:$ Dies bedeutet, dass die im Sternentstehungszeitskala $\tau_{\mathrm{dep}}=\tau_{\mathrm{ff}} / \epsilon_{\mathrm{ff}}$ eine Konstante (siehe Gleichung 3.10) ist. Eine solche lineare KS-Relation wird für kaltes, molekulares Gas beobachtet, wobei die inferierte Sternentstehungszeitskala für weniger dichtes molekulares Gas (auf Skalen von einigen hundert Parsec gemittelt) im Bereich von $\tau_{\text {dep }} \approx 2$ Ga liegt. Für einzelne Wolken oder den dichtesten Teil des molekularen Gases auf galaktischen Skalen hingegen wurde ein Wert von $\tau_{\text {dep }} \lesssim 100$ Ma ermittelt.

- $\alpha_{\mathrm{KS}} \approx 2$ : Diese KS-Relation für das neutrale Gas wurde in Galaxien, die gerade einen Sternentstehungsausbruch (star burst) erleben, abgeleitet. Der vermutete Mechanismus dahinter ist, dass dort Kollisionen von Gaswolken (cloud-cloud collisions; CCC) der wichtigste Auslöser für Sternentstehung sind.

Es ist anzunehmen, dass sich Sterne aus kaltem, besonders dichtem, molekularem Gas mit einer Rate bilden, die proportional zur verfügbaren Menge dieses Gases ist. Es handelt sich hierbei um das Gas, welches aktiv am Sternentstehungsprozess beteiligt ist und beispielsweise in Beobachtungen von Linienübergängen des Blausäuremoleküls (HCN) erfasst werden kann (zum Beispiel Gao u. Solomon, 2004). Dieses Gasreservoir wird mit einer Rate aufgefüllt, die proportional ist zum wesentlich größeren Vorrat an weniger dichtem, aber auch kaltem und molekularem Gas, das im Licht von Spektrallinien der Kohlenmonoxids strahlt. Deshalb beobachten zum Beispiel Bigiel u. a. (2011) eine lineare KS-Relation für molekulares Gas mit einer wesentlich längeren Sternentstehungszeitskala als beispielsweise Evans u. a. (2009); Murray (2011). Die Rate, mit der nun der letztere Gasvorrat aufgefüllt wird, hängt stark von der galaktischen Umgebung ab. Ist die Galaxie gasreich, insbesondere wenn sie sich gerade inmitten eines Verschmelzungsprozesses befindet, so wird die Produktion kalten, molekularen Gases aus wärmerem neutralem Gas maßgeblich durch Kollisionen von Gaswolken verursacht, sodass sich $\alpha_{\mathrm{KS}} \approx 2$ ergibt. In gasärmeren Umgebungen sind hingegen gravitative Instabilitäten im ISM der dominante 
Prozess, der zur Bildung von molekularem Gas führt. Da diese Instabilitäten auf Zeitskalen von $\tau_{\mathrm{ff}}$ arbeiten, resultiert $\alpha_{\mathrm{KS}} \approx 1,5$.

\subsubsection{Stellare Rückwirkung}

Unter dem Begriff der stellaren Rückwirkung (stellar feedback) werden alle Vorgänge zusammengefasst, mit denen ein Stern über seine Lebensspanne auf das umgebende ISM einwirkt. Dazu gehören ionisierende Strahlung, stellare Winde und in besonderem Maße explosive Ereignisse (Supernovae; SN), um nur die wichtigsten zu nennen. Die nuklearen Reaktionen in Inneren eines Sterns gleichen durch ihre Energiefreisetzung den Strahlungsverlust an seiner Oberfläche nur solange aus, bis die Brennstoffvorräte aufgezehrt sind. Danach vergeht der Stern abhängig von seiner Masse eventuell nach weiteren Brennphasen, in denen schwere Elemente jenseits von Helium erbrütet werden können. ${ }^{5}$ Wenn er massereich genug war explodiert er als SN vom Typ II ${ }^{6}$ die mit einer erheblichen Freisetzung von Energie und einst stellarem Material in das ISM einhergeht. Durch SN, aber auch durch stellare Winde wird das ISM mit Metallen angereichert. Die von Sternen abgegebene Strahlung, die stellaren Winde und nicht zuletzt die SN treiben viele dynamische Vorgänge im ISM maßgeblich an und wirken auch auf den Sternbildungsvorgang in komplexer Weise ein. SNe können beispielsweise eine ehemalig aktive Sternentstehungsregion komplett zerstören, indem das Gas aufgeheizt und auseinander getrieben wird. Andererseits kann eben dies an anderer Stelle zur Verdichtung des Gases und der Bildung einer neuen Sternentstehungsregion führen.

\footnotetext{
${ }^{5}$ Der Entwicklungsweg, den ein Stern durchläuft, hängt im Wesentlichen nur von seiner Anfangsmasse und seiner initialen Metallizität ab (siehe beispielsweise Kippenhahn u. a., 2012). So haben massereichere Sterne eine viel kürzere Phase des Wasserstoffbrennens im Kern als masseärmere. Beispielsweise ist die Sonne bereits $\sim 4,5$ Ga alter Hauptreihenstern, denn sie verbrennt in ihrem Inneren noch immer Wasserstoff zu Helium. Während sie noch für weitere $\sim 4,5$ Ga einen Wasserstoffvorrat besitzt, ist die Hauptreihenphase eines Sterns mit der zehnfachen Masse $\left(10 \mathrm{M}_{\odot}\right)$ nur wenige 10 Ma lang, da solche Sterne ihren Brennstoff wesentlich wegen höheren Dichten und höherer Temperaturen im Kern effizienter umsetzten können. Sterne mit geringerer Metallizität sind an ihrer Oberfläche heißer als metallreichere, da höher metallangereichertes Gas besser an die von Innen kommende Strahlung koppelt, was den Stern aufbläht. Außerdem wirken Metalle besonders in massereichen Sternen als Katalysator, indem sie den sogenannten CNO-Zyklus ermöglichen, der bei höheren Temperaturen effizienter ist als die pp-Kette.

${ }^{6}$ Eigentlich handelt es sich bei einer SN des Typs II um eine Implosion, bei der der Kern des Sterns in sich zusammen fällt. Die Explosion entsteht erst durch das Zurückprallen der nachstürzenden Hüllen am kollabierenden Kern, wobei der Strahlungsdruck der während des Kollapses des Kerns äußerst zahlreich entstehenden Neutrinos beim endgültigen Abblasen der Hülle wesentlichen Anteil hat.
} 


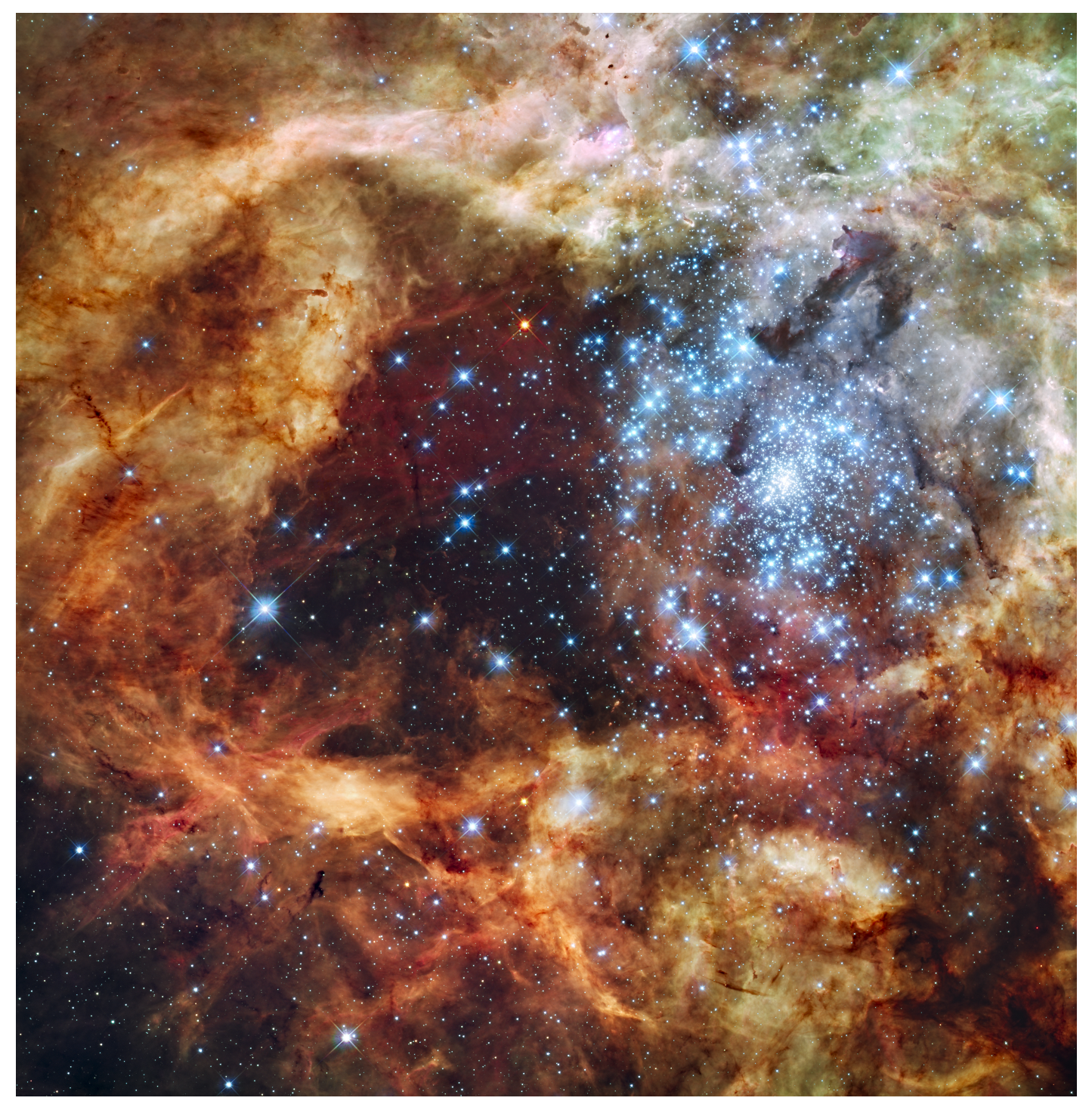

Abbildung 3.2: Einige zehn Parsec großer Ausschnitt des Zentrums von NGC 2070 - auch Tarantel-Nebel oder 30 Doradus genannt: Der gesamte Nebel hat eine Ausdehnung von einigen hundert Parsec und liegt circa $49 \mathrm{kpc}$ entfernt in der Großen Magellan'schen Wolke, dem größten Satelliten der Milchstraße. Zu sehen ist ein junger Sternhaufen, R136, in dessen Licht das ihn blasenartig umgebende Gas in dem viele wolkige und filamentartige Strukturen erkennbar sind, erleuchtet. Dichte, staubreiche Wolken im Vordergrund erscheinen als dunkle oder bräunliche Schatten. Kompositbild aus Beobachtungsdaten in verschieden Wellenlängenbereichen aufgenommen vom Hubble Space Telecope (Bild: NASA, ESA, F. Paresce (INAF-IASF), R. O'Connell (U. Virginia), and the HST WFC3 Science Oversight Committee). 


\section{Kapitel 4}

\section{Modell des turbulenten ISM}

In diesem Kapitel wird das maßgeblich von mir entwickelte Ein-Zonen-Modell für das turbulente, sternbildende ISM vorgestellt und in Grundzügen beschrieben, das in der Fachzeitschrift Monthly Notices of the Royal Astronomical Society (MNRAS) als Braun u. Schmidt (2012, fortan BS12) veröffentlicht wurde. Für eine detaillierte Beschreibung des Modells sowie eine ausführliche Diskussion des Verhaltens seiner dynamischen Lösungen und stationären Zustände sei hier auf BS12 im Anhang A.1 verwiesen. In diesem Modell werden unterschiedliche Ansätze zur Beschreibung von Teilaspekten von Zustand und Dynamik des sternbildenden ISM miteinander zu einem effektiven Modell für Skalen von einigen 10 pc verbunden, das insbesondere die Effekte kleinskaliger Turbulenz in seiner Dynamik berücksichtigt. Dieses Modell diente im weiteren Verlauf meiner Arbeit als Basis von MIST (beschrieben in Abschnitt 5.1.3). Letzteres fand Verwendung in LES idealisierter Scheibengalaxien (siehe Kapitel 5).

Im Folgenden stelle ich zunächst kurz das Konzept des Modells (Abschnitt 4.1) und die Modellgleichungen (Abschnitt 4.2) vor, um dann auf einige der Ergebnisse der Gleichgewichtslösungen des Modells einzugehen (Abschnitt 4.3) und dieses Kapitel mit Vergleichen zu Beobachtungen abzuschließen (Abschnitt 4.4).

\subsection{Konzeption des Modells}

Im ISM liegt Gas gleichzeitig und nebeneinander auf relativ engem Raum in zum Teil äußerst unterschiedlichen Zuständen vor, die im Allgemeinen als Phasen bezeichnet werden. Diese lassen sich durch Dichte und Temperatur charakterisieren, aber auch durch ihre jeweilige chemische Zusammensetzung. So liegt zum Beispiel Wasserstoff in kaltem, dichtem Gas vorwiegend in Form von Molekülen $\left(\mathrm{H}_{2}\right)$ vor, während in wärmerem, weniger dichtem Gas eher neutraler, atomarer Wasserstoff (HI), und in noch heißerem, dünnem Gas bevorzugt ionisierter Wasserstoff (HII) anzutreffen ist. Bedingt durch den jeweiligen Zustand, in dem sich das Gas lokal befindet, sind un- 
terschiedliche physikalische Prozesse in unterschiedlichem Maße wirksam. Diese Prozesse, aber auch die Wechselwirkung mit der Umgebung, insbesondere das Werden und Vergehen von Sternen, führen zu einem dynamischen Austausch von Material und Energie zwischen den verschiedenen Phasen.

Die Grundidee hinter dem ISM-Modell ist, die Mehrphasen-Natur des ISM mithilfe zweier unterschiedlicher Fraktionen abzubilden. Der Gasinhalt eines Referenzvolumens wird aufgeteilt, ähnlich wie es bereits Springel u. Hernquist (2003) taten. Den verschiedenen Fraktionen - oder Phasen - werden bestimmte Eigenschaften zugeschrieben. Grundlegend wird das Gas zunächst in eine kalte und eine warme Fraktion unterteilt. Dabei handelt es sich einerseits um warmes, meist neutrales Gas und andererseits um kaltes, dichtes, möglicherweise molekulares Gas, das dazu neigt, Klumpen auszuformen, die wiederum vom warmen Medium umgeben sind.

\subsubsection{Phasen}

Das Modell berücksichtigt exemplarisch die bereits vorgestellten zwei Phasen, parametrisiert durch ihre Partialdichten mit $\rho_{\mathrm{c}}+\rho_{\mathrm{w}}=\rho .^{1}$ Innerhalb des Referenzvolumens $\ell^{3}$ können beide nebeneinander existieren. Die klumpige, kalte Phase $\rho_{\mathrm{w}}$ hat eine als im Mittel konstant angenommene Temperatur $T_{\mathrm{c}}=10 \ldots 100 \mathrm{~K}$, also auch eine feste spezifische thermische Energie $e_{\mathrm{c}}$ (in BS12 $u_{\mathrm{c}}$ ), während die warme Phase eine variable Temperatur $T_{\mathrm{w}}$ besitzt. Beide Phasen haben gemeinsam eine Metallizität $Z$ sowie eine auf der Längenskala $\ell$ abgemessene spezifische Energie $K$ (in BS12 $e_{\mathrm{t}}$ ) von ungeordneten, turbulenten Gasbewegungen auf kleineren Skalen. Anders als in Springel u. Hernquist (2003) oder Murante u. a. (2010) gibt das Verhältnis der Partialdichten nicht ebenfalls das Verhältnis der Volumenfüllfaktoren wieder, sondern ausschließlich das Massenverhältnis der beiden Phasen. Die Phasen dürfen unterschiedliche mittlere Dichten, $\rho_{\mathrm{c}, \mathrm{pa}}$ und $\rho_{\mathrm{w}, \mathrm{pa}}$, aufweisen. Unter der Annahme, dass zwischen den beiden Phasen ein Gleichgewicht der effektiven Drücke

$$
P_{\mathrm{w}, \mathrm{eff}} \stackrel{!}{=} P_{\mathrm{c}, \mathrm{eff}}
$$

- der Summen der thermischen und turbulenten Drücke - auf der Längenskala $\ell_{c}$ der Klumpen der kalten Phase mit

$$
\ell_{\mathrm{c}} \simeq \lambda_{\mathrm{J}, \mathrm{c}} \equiv c_{\mathrm{c}}\left(\frac{\pi}{\gamma G \rho_{\mathrm{c}, \mathrm{pa}}}\right)^{1 / 2}=\left(\frac{\pi(\gamma-1) u_{\mathrm{c}}}{G \rho_{\mathrm{c}, \mathrm{pa}}}\right)^{1 / 2}
$$

\footnotetext{
${ }^{1}$ Wie in BS12 bezeichnen Symbole mit Subskript 'c' oder 'w' Größen, die der kalten, beziehungsweise der warmen Phase zuzuordnen sind, während sich solche ohne entsprechendes Subskript auf den gesamten Gasinhalt des Referenzvolumens beziehen.
} 
herrscht, können die jeweiligen Dichten bestimmt werden. $\lambda_{\mathrm{J}, \mathrm{c}}$ ist die thermische Jeanslänge des kalten Gases. Der effektive Druck der kalten Phase ergibt sich aus

$$
P_{\mathrm{c}, \mathrm{eff}}=\rho_{\mathrm{c}, \mathrm{pa}} \sigma_{\mathrm{c}, \mathrm{eff}} \equiv \rho_{\mathrm{c}, \mathrm{pa}}\left(\frac{c_{\mathrm{c}}^{2}}{\gamma}+\sigma_{\mathrm{c}}^{2}\right)=(\gamma-1) \rho_{\mathrm{c}, \mathrm{pa}} e_{\mathrm{c}}\left(1+\frac{\gamma}{3} \mathcal{M}_{\mathrm{c}}^{2}\right)
$$

wobei

$$
\sigma_{\mathrm{c}}^{2}=\frac{2 K}{3}\left(\frac{\ell_{\mathrm{c}}}{\ell}\right)^{2 \eta}
$$

die effektive Geschwindigkeitsdispersion in der kalten Phase auf der Längenskala von $\ell_{\mathrm{c}}$ ist. $K$ ist die spezifische kinetische Energie in turbulenten, ungeordneten Bewegungen des Gases unterhalb der Längenskala $\ell$, wobei das Mittel der mit diesen Bewegungen assoziierten Impulse über das Referenzvolumen $\ell^{3}$ verschwindet. Die Konstruktion der turbulenten Energie $K$ ist weitestgehend analog zur Konstruktion der thermischen Energie $e$ in der Hydrodynamik (siehe Kapitel 2.1.2). Die bereits im Impulsraum gemittelten Geschwindigkeiten $\overline{\mathbf{u}}$ sowie die zugehörige kinetische Energie $E-e$ werden hierbei nochmals lokal im Ortsraum (über das Referenzvolumen $\left.\ell^{3}\right)$ gemittelt, sodass $\rho K=\langle\rho(E-e)\rangle_{\ell}-\langle\rho \overline{\mathbf{u}}\rangle_{\ell}^{2} /\left(2\langle\rho\rangle_{\ell}\right)$ gilt $^{2}$.

Der effektive Druck der warmen Phase ist durch

$$
P_{\mathrm{w}, \mathrm{eff}}=\rho_{\mathrm{w}, \mathrm{pa}} \sigma_{\mathrm{w}, \mathrm{eff}} \equiv \rho_{\mathrm{w}, \mathrm{pa}}\left(\frac{c_{\mathrm{w}}^{2}}{\gamma}+\sigma_{\mathrm{w}}^{2}\right)=(\gamma-1) \rho_{\mathrm{w}, \mathrm{pa}}\left(e_{\mathrm{w}}+\sigma_{\mathrm{c}}^{2}\right)
$$

gegeben. Das Gleichungssystem aus den Gleichungen 4.1 bis 4.5 ist iterativ gut lösbar.

Die Annahme, die kalte Phase liege in Klumpen vom Durchmesser einer thermischen Jeans-Länge $\lambda_{\mathrm{J}, \mathrm{c}}$ vor, stellt eine grobe Vereinfachung der komplizierten, filamentartigen Strukturen dar, in welchen das kalte Gas tatsächlich anzutreffen ist. $\ell_{c}$ ist als eine typische oder charakteristische Längenskala anzusehen, mit deren Hilfe andere wichtige Eigenschaften - wie die effektive Energieinjektionsskala der Turbulenz im sternbildenden Gas oder die effektive optische Tiefe - abgeschätzt werden können. Andere charakteristische Skalen, die zum Beispiel den effektiven Druck oder die Heiz-/Kühlraten berücksichtigen, wären ebenfalls mögliche Kandidaten gewesen. Doch diese führen zu ungünstigem, beziehungsweise unphysikalischem Verhalten des Modells, stellen also keine brauchbare Alternative zu $\lambda_{\mathrm{J}, \mathrm{c}}$ dar. So sind zum Beispiel die Kühlzeitskalen in der Regel kurz. Dies bedeutet für eine mittels zugehörigem Schallhorizont definierte Längenskala, dass auf dieser kurzen Längenskala Turbulenz aufgrund ihrer Skalierungseigenschaften eine eher untergeordnete Rolle spielt, was sowohl Beobachtungen als auch der Konzeption dieses Modells widerspricht. Kalte Phase entsteht aus warmer Phase über radiatives Kühlen unter bestimmten

\footnotetext{
${ }^{2}$ Die entsprechende Mittelung wurde hierbei durch Anwendung des Operators $\langle\cdot\rangle_{\ell}$ ausgeführt.
} 
Bedingungen. Im Modell geschieht dies, wenn einerseits eine Temperatur $T_{\mathrm{TI}}=$ $1.5 \times 10^{4} \mathrm{~K}$ unterschritten wird und andererseits die mittlere Dichte $\rho_{\mathrm{w}, \mathrm{pa}}$ der warmen Phase einen Wert von 0,1 Partikeln pro $\mathrm{cm}^{3}$ überschreitet. Der Austausch von Material und Energie wird kurz in Abschnitt 4.2 beschrieben.

\subsubsection{Sternentstehung}

Sterne formen sich aus lokal besonders dichten Gasansammlungen innerhalb kalten, molekularen Gases durch gravitativ getriebenen Kollaps. Folgende Gleichung für die Sternentstehungsrate $\dot{\rho}_{\mathrm{s}}$, die in ähnlicher Form zum Beispiel auch von Gnedin u. a. (2009) verwendet wurde, spiegelt dies wieder:

$$
\dot{\rho}_{\mathrm{s}}=\frac{\epsilon_{\mathrm{PN}} f_{\mathrm{H}_{2}} \rho_{\mathrm{c}}}{\tau_{\mathrm{c}, \mathrm{ff}}} .
$$

Hier gehen neben der Partialdichte des kalten Gases $\rho_{\mathrm{c}}$, die Freifall-Zeitskala

$$
\tau_{\mathrm{c}, \mathrm{ff}}=\sqrt{\frac{3 \pi}{32 G \rho_{\mathrm{c}, \mathrm{pa}}}}
$$

in der kalten Phase und der relative Massenanteil $f_{\mathrm{H}_{2}}$ des abgeschirmten, molekularen Gases innerhalb der kalten Phase, der mittels eines Strømgren-Ansatzes ${ }^{3}$ ähnlich dem von McKee u. Krumholz (2010) ermittelt wird, sowie die Sternentstehungseffizienz $\epsilon_{\mathrm{PN}}$ pro Freifallzeit $\tau_{\mathrm{c}, \mathrm{ff}}$ ein.

Im Gegensatz zu den gängigen Modellen, die auf eine Anwendung in Simulationen von Galaxien inklusive ihres Umfeldes zugeschnitten sind, ist die Sternentstehungseffizienz $\epsilon_{\mathrm{PN}}$ keine vorab aufgrund von Erfahrungswerten oder modellhafter Annahmen gewählte Konstante, sondern eine dynamische Größe, die aus dem turbulenten Mehrphasenzustand des Gases innerhalb eines Referenzvolumens abgeleitet wird. Die Dynamik im kalten Gas wird maßgeblich durch turbulente Bewegungen bestimmt, die aufgrund der geringen Temperatur von weniger als $100 \mathrm{~K}$ weit im Überschallbereich liegen. Dadurch ergeben sich im Bereich von aufeinander treffenden Schockfronten teils erhebliche lokale Verdichtungen des Gases. An anderer Stelle liegt das Gas verdünnt vor. Für die Sternentstehung sind jene Verdichtungen von Interesse, welche durch ihre eigene Schwerkraft zusammengehalten werden. Nach Padoan u. Nordlund (2011) lässt sich die relative kritische Überdichte $x_{\text {crit }}=\rho_{\text {crit }} / \rho_{\text {c,pa }}$ einer solchen Verdichtung durch die turbulente Machzahl $\mathcal{M}_{\mathrm{c}}$ und den Virialparameter $\alpha_{\text {vir }}$ der kalten

\footnotetext{
${ }^{3}$ Dieser Gleichgewichtsansatz ist nur gültig, wenn sich das chemische Reaktionsgleichgewicht binnen ausreichend kurzer Zeit einstellen kann. Dies betrifft vor Allem die $\mathrm{H}_{2}$-Produktionsraten. Im Modell hängt diese hauptsächlich von der katalytischen Bildung von $\mathrm{H}_{2}$ an interstellarem Staub ab. Dies stellt eine Einschränkung an die Metallizität des Gases dar. Sie sollte wenigstens im Bereich von $Z \sim Z_{\odot}$ liegen, um diese Bedingung sicher zu erfüllen.
} 
Klumpen abschätzen:

$$
x_{\text {crit }}=0.0371 \alpha_{\mathrm{vir}} \mathcal{M}_{\mathrm{c}}^{2}=\frac{0.0742}{G \gamma(\gamma-1) \rho_{\mathrm{c}, \mathrm{pa}} e_{\mathrm{c}}} \cdot \frac{K^{2}}{\ell_{\mathrm{c}}^{2(1-2 \eta)} \ell^{4 \eta}} .
$$

Der Virialparameter

$$
\alpha_{\mathrm{vir}} \equiv \frac{5 \sigma_{\mathrm{c}}^{2} \ell_{\mathrm{c}}}{6 G M_{\mathrm{c}}}=\frac{10 K\left(\ell_{\mathrm{c}} / \ell\right)^{2 \eta}}{\pi G \ell_{\mathrm{c}}^{2} \rho_{\mathrm{c}, \mathrm{pa}}}
$$

ist ein Maß für die relative Stärke von Gravitation und dagegen stabilisierender Bewegungen innerhalb einer Gasansammlung. Die Machzahl der Turbulenz hingegen gibt wieder, wie stark Schockwellen im Gas ausgeprägt sind, und dient zusammen mit dem Parameter $b$ als Maß für die statistische Breite der Verteilung der Gasdichten ${ }^{4}$. Eine log-normale Verteilung der Gasdichten im kalten Medium um den Mittelwert $\rho_{\mathrm{c}, \mathrm{pa}}$ mit Varianz

$$
\sigma_{\mathrm{c}, \mathrm{pdf}}^{2} \approx \ln \left(1+b^{2} \mathcal{M}_{\mathrm{c}}^{2}\right)
$$

vorausgesetzt, kann der Massenanteil von der gesamten Gasmasse, der sich innerhalb einer Freifallzeit in gravitativ gebundene, kollabierende Objekte wandelt, durch das Integral dieser Verteilung oberhalb der kritischen Überdichte $x_{\text {crit }}$ ausgedrückt werden. Durch Hinzufügen einer Effizienz $\epsilon_{\text {core }}$, die angibt, wie viel der ursprünglichen Masse eines solchen Objektes schließlich in Sternen endet und nicht während des prästellaren Kollapses durch Winde abgeblasen wird, ergibt sich $\epsilon_{\mathrm{PN}}$ als

$$
\epsilon_{\mathrm{PN}}=\epsilon_{\mathrm{core}} \frac{1}{2}+\frac{1}{2} \operatorname{erf}\left[\frac{\sigma_{\mathrm{c}, \mathrm{pdf}}^{2}-2 \ln \left(x_{\mathrm{crit}}\right)}{2^{3 / 2} \sigma_{\mathrm{c}, \mathrm{pdf}}}\right] .
$$

An dieser Stelle möchte ich hervorheben, dass diese Modellierung der Sternentstehung keine bestimmte Form der KS-Relation vorgibt, sondern sich diese später sozusagen 'natürlich' als ein Ergebnis des Modells durch Selbstregulation ergibt.

\subsubsection{Stellare Rückwirkung}

Im Rahmen dieses Modells nehme ich an, dass durch den Sternbildungsprozess eine Population von Sternen hervorgebracht wird, deren statistische Verteilung $\mathrm{d} N_{*} / \mathrm{d} m_{*}$ (IMF: (stellar) initial mass function) der initialen individuellen Sternenmassen $m_{*}$ der von Chabrier (2001) beschriebenen entspricht. Diese nimmt im für stellare Rückwirkung (englisch: stellar feedback) interessanten Massenbereich jenseits von $8 \mathrm{M}_{\odot}$ die Form eines Potenzgesetzes mit Exponenten -2,3 an. Aus den Sternmassen und der Metallizität lässt sich unter Verwendung von Sternentwicklungsmodellen die

\footnotetext{
${ }^{4} b=[1 / 3 \ldots 1]$ gibt die Art der die Turbulenz treibenden Moden an - also ob solenoidale oder kompressive Moden vorherrschen. Dabei steht ein Wert von $b=1$ für rein kompressives Treiben, und $b=1 / 3$ für rein solenoidales, siehe Federrath u. a. (2010).
} 
Entwicklung der Sterne berechnen. So verwende ich eine Parametrisierung $m_{*}\left(t^{\prime}, Z\right)$ von Raiteri u. a. (1996) basierend auf Sternentwicklungsmodellen der Padua Gruppe (Alongi u.a., 1993; Bressan u.a., 1993; Bertelli u.a., 1994), um die Masse der Sterne zu bestimmen, die nach Ablauf ihrer Lebensdauer in einem Alter von $t^{\prime}$ vergehen und aus Gas mit Metallizität $Z$ entstanden sind. Sterne im Massenbereich zwischen $8 \mathrm{M}_{\odot}$ und $40 \mathrm{M}_{\odot}$ enden in energetischen Explosionen, die als SN vom Typ II bezeichnet werden. Diese werden im Modell statistisch berücksichtigt. Das heißt, dass eine Sternenpopulation gemäß ihres Alters kontinuierlich einen Teil ihrer Masse (insgesamt etwa 15\% der anfänglichen Masse) an das umgebende Gas abgibt, wobei zusätzlich erhebliche Mengen von schweren Elementen und Energie in das ISM eingetragen werden. Der entsprechende Quellterm für die ins ISM abgegebene Masse ist eine mit der Sternenmasse $m_{*}$ gewichtete Faltung der IMF $\mathrm{d} N_{*} / \mathrm{d} m_{*}$ mit der Sternentstehungsrate $\dot{\rho}_{\mathrm{s}}$ früherer Zeiten :

$$
\dot{\rho}_{\mathrm{s}, \mathrm{fb}}(t)=\int_{t_{\mathrm{b}}}^{t_{\mathrm{e}}} \dot{\rho}_{\mathrm{s}}\left(t-t^{\prime}\right) \frac{1}{M_{*}} \frac{\mathrm{d} N_{*}}{\mathrm{~d} m_{*}} \frac{\mathrm{d} m_{*}}{\mathrm{~d} t^{\prime}} \mathrm{d} t^{\prime}
$$

wobei $M_{*}$ die Normierungskonstante der IMF ist, und die Integrationsgrenzen $t_{\mathrm{b}}$ und $t_{\mathrm{e}}$ der Lebensdauer von Sternen mit Anfangsmassen von 40 beziehungsweise 8 $M_{\odot}$ entsprechen.

Die bereits erwähnte Metallanreicherung des Gases wird mit der folgenden Rate beschrieben:

$$
\left.\frac{\mathrm{d}(Z \rho)}{\mathrm{d} t}\right|_{\mathrm{s}, \mathrm{fb}}=\left(\zeta_{\mathrm{m}} \dot{\rho}_{\mathrm{s}, \mathrm{fb}}+\int_{t_{\mathrm{b}}}^{t_{\mathrm{e}}} Z\left(t-t^{\prime}\right) \dot{\rho}_{\mathrm{s}}\left(t-t^{\prime}\right) \frac{1}{M_{*}} \frac{\mathrm{d} N_{*}}{\mathrm{~d} m_{*}} \frac{\mathrm{d} m_{*}}{\mathrm{~d} t^{\prime}} \mathrm{d} t^{\prime}\right)
$$

wobei $\zeta_{\mathrm{m}}$ der relative Massenanteil der SN-Auswürfe, der aus in den Sternen neu erzeugten Metallen besteht.

Für den Energieeintrag ins ISM wird angenommen, dass dieser proportional zum Massenauswurf ist, die Auswürfe tragen also eine konstante spezifische Energie $e_{\mathrm{SN}}$ in sich. Die Anzahl $n_{\mathrm{SN}}$ von SN-Ereignissen vom Typ II, die pro Sonnenmasse $\mathrm{M}_{\odot}$ einer Sternenpopulation stattfinden, errechnet sich aus

$$
n_{\mathrm{SN}}=\frac{1}{M_{*}} \int_{8 \mathrm{M}_{\odot}}^{40 \mathrm{M}_{\odot}} \frac{\mathrm{d} N_{*}}{\mathrm{~d} m_{*}} \mathrm{~d} m_{*} .
$$

Daraus ergibt sich mit der pro SN freigesetzten Energie ${ }^{5} E_{\mathrm{SN}}=10^{51} \mathrm{erg}$ und der Gesamtmasse der Ejekta

$$
M_{\mathrm{SN}}=\frac{1}{M_{*}} \int_{8 \mathrm{M}_{\odot}}^{40 \mathrm{M}_{\odot}} m_{*} \frac{\mathrm{d} N_{*}}{\mathrm{~d} m_{*}} \mathrm{~d} m_{*}
$$

\footnotetext{
${ }^{5}$ Es handelt sich hierbei nicht um die gesamte freigesetzte Energie einer SN $\left(\sim 10^{53} \mathrm{erg}\right)$, sondern um den Anteil, der in den ausgestoßenen Hüllen des ehemaligen Sterns steckt. Der Rest wird hauptsächlich in Form von Neutrinos abgegeben, die praktisch keine Wirkung auf das ISM haben.
} 
die mittlere spezifische Energie $e_{\mathrm{SN}}=n_{\mathrm{SN}} E_{\mathrm{SN}} / M_{\mathrm{SN}}$ des ausgestoßenen Gases. Diese Energie wird auf die Budgets der turbulenten Energie $K$ und der thermischen Energie $e_{\mathrm{w}}$ des warmen Gases verteilt, wobei $K$ ein Anteil $\epsilon_{\mathrm{SN}}=0.085$ zukommt, dessen Wert sich aus den Berechnungen von Thornton u. a. (1998) ableitet.

Abgesehen von den bereits vorgestellten Auswirkungen von SN wird im Modell berücksichtigt, dass die Explosionen - oder vielmehr ihre Expansionswellen - in der Lage sind kaltes Gas zu evaporieren, zu heizen, und der warmen Phase unterzumischen. Dies wird mittels eines an die Arbeit von McKee u. Ostriker (1977) angelehnten Austauschterms bewerkstelligt:

$$
\left.\frac{\mathrm{d} \rho_{\mathrm{c}}}{\mathrm{d} t}\right|_{\mathrm{SN}}=A \dot{\rho}_{\mathrm{s}, \mathrm{fb}}
$$

wobei

$$
A=A_{0}\left(\frac{\rho_{\mathrm{w}}}{\rho_{\mathrm{w}, 0}}\right)^{-\frac{4}{5}}\left(\frac{l_{\mathrm{c}}}{l_{\mathrm{c}, 0}}\right)^{-\frac{6}{5}}\left(\frac{V_{\mathrm{c}}}{V_{\mathrm{c}, 0}}\right)^{\frac{3}{5}}
$$

mit den Skalierungswerten

$$
\begin{aligned}
T_{0} & =T_{\mathrm{TI}}=1.5 \times 10^{4} \mathrm{~K}, \\
u_{0} & =k_{B} T_{0} / \mu m_{H}(\gamma-1), \\
A_{0} & =e_{\mathrm{SN}} / 2 u_{0}, \\
\rho_{0} & =\mu m_{\mathrm{H}} \times 10.0 \mathrm{~cm}^{-3}, \\
\rho_{\mathrm{c}, 0} & =10^{-3} \rho_{0}, \\
\rho_{\mathrm{w}, 0} & =\rho_{0}-\rho_{\mathrm{c}, 0}, \\
l_{\mathrm{c}, 0} & =\lambda_{\mathrm{J}, \mathrm{c}}\left(u_{\mathrm{c}}, \rho_{\mathrm{c}, \mathrm{pa}, 0}\right) .
\end{aligned}
$$

Neben den SN Typ II wird im Modell auch das Heizen der Gases durch ionisierende Strahlung von jungen heißen Sternen berücksichtigt. Die sogenannte LymanKontinuumsstrahlung ist für die Entstehung von HII-Regionen ${ }^{6}$ verantwortlich, und heizt dabei das Gas auf. Die Quellen dieser Strahlung sind heiße, massereiche Sterne, deren Emissionsrate $\dot{N}_{\text {Lyc }}$ ionisierender Photonen mittels einer Fitfunktion an Daten des Spektralsyntheseprogramms galev (Kotulla u.a., 2009) abgeschätzt wird. Hierbei wird angenommen, dass jedes Photon bereits innerhalb des Referenzvolumens absorbiert wird, wobei ein Wasserstoffatom ionisiert wird und im Mittel nur $x_{\text {Lyc }}=0,1 \mathrm{eV}$ Energie effektiv als Wärme im Gas verbleiben. Der Großteil der Photonenenergie wird bei Linienübergängen im Zuge Rekombination wieder abgestrahlt, die aufgrund der hohen Dichten in HII-Regionen der Ionisation rasch nachfolgt.

\footnotetext{
${ }^{6}$ HII-Regionen sind Bereiche des ISM, die nicht durch thermische Stöße, sondern durch den von energiereicher, elektromagnetischer Strahlung ausgelösten Photoeffekt ionisiert wurden.
} 


\subsection{Modellgleichungen}

Wie bereits ausgeführt, entsteht Kalte Phase aus warmer Phase über radiatives Kühlen unter bestimmten Bedingungen. Im Modell wird Masse entsprechend der Menge von durch Kühlen verlorenen Energie und der Differenz der spezifischen thermischen Energien der beiden Phasen von der warmen in die kalte Phase transferiert. Zusätzlich wird dabei durch die zwangsläufig auftretenden lokalen Druckunterschiede bei der Phasentrennung ein Teil der thermische Energie der warmen Phase - proportional zur Kühlrate $\Lambda_{\text {eff }}$ - in turbulente Energie umgewandelt. Gas in der kalten Phase wiederum kann in die warme Phase durch Evaporation und Untermischen überführt werden. Prozesse, die zu einer Erwärmung der kalten Phase führten, werden modelliert, indem Material entsprechend der Heizraten und der Differenz der spezifischen inneren Energien der Phasen von der kalten in die warme Phase übertragen wird. Von größerer Wichtigkeit ist jedoch die Evaporation von kaltem Gas durch SN-Explosionen, deren sich ausbreitende Schockfronten in der Lage sind, das Gas in einem Referenzvolumen effektiv zu durchmischen.

Dies sind die partiellen Differentialgleichungen, die das Verhalten meines Modells für das turbulente, sternbildende ISM festlegen:

- Die Rate $\dot{\rho}_{\mathrm{c}}$ der partialen Dichte der kalten Phase setzt sich aus den Termen für Sternentstehung und für SN-Evaporation sowie dem Massentransfer wegen Kühlung in der warmen Phase, Heizen in der kalten Phase aufgrund von Lyman-Kontinuumsstrahlung, turbulenter Dissipation und Kollisionen von kalten Klumpen (CCC) zusammen ${ }^{7}$.

$$
\begin{aligned}
\dot{\rho}_{\mathrm{c}}= & -\dot{\rho}_{\mathrm{s}}-A \dot{\rho}_{\mathrm{s}, \mathrm{fb}}-\frac{1}{e_{\mathrm{w}}-e_{\mathrm{c}}} \\
& \times\left[-\left(1-f_{\mathrm{TI}}\right) \rho_{\mathrm{w}} \Lambda_{\mathrm{eff}}+\frac{\dot{N}_{\mathrm{Lyc}} x_{\mathrm{Lyc}} \rho_{\mathrm{c}}}{\rho}+\frac{C_{\epsilon} K^{3 / 2} \rho_{\mathrm{c}}}{l}\right] \\
& -\frac{36 \epsilon_{\mathrm{CCC}} \rho_{\mathrm{c}, \mathrm{pa}} V_{c}^{2}}{\pi l_{\mathrm{c}}}\left[2 e_{\mathrm{t}}\left(1-\left(\frac{l_{\mathrm{c}}}{l}\right)^{2 \eta}\right)\right]^{1 / 2}
\end{aligned}
$$

- Die Rate $\dot{\rho}_{\mathrm{w}}$ der partialen Dichte der warmen Phase ergibt sich wegen Massenerhaltung aus der Sternentstehungsrate und $\dot{\rho}_{\mathrm{c}}$.

$$
\dot{\rho}_{\mathrm{w}}=-\dot{\rho}_{\mathrm{c}}-\dot{\rho}_{\mathrm{s}, \mathrm{eff}}
$$

- Die effektive Sternentstehungsrate $\dot{\rho}_{\mathrm{s}, \text { eff }}$ ergibt sich als Differenz von der Rate $\dot{\rho}_{\mathrm{s}}$, mit der neue Sterne gebildet werden, und der SN-Auswurfrate $\dot{\rho}_{\mathrm{s}, \mathrm{fb}}$.

$$
\dot{\rho}_{\mathrm{s}, \mathrm{eff}}=\dot{\rho}_{\mathrm{s}}-\dot{\rho}_{\mathrm{s}, \mathrm{fb}}
$$

\footnotetext{
${ }^{7}$ Für eine detaillierte Erklärung der einzelnen Terme siehe BS12 im Anhang A.1.
} 
- Die Änderungsrate $\dot{e}_{\mathrm{w}}$ der spezifischen thermischen Energie der warmen Phase setzt sich aus den Termen für die Abgabe thermischer Energie durch SN (1 $\left.\epsilon_{\mathrm{SN}}\right) \dot{\rho}_{\mathrm{s}, \mathrm{fb}} e_{\mathrm{SN}}$, die durch SN ausgelöste Evaporation $A \dot{\rho}_{\mathrm{s}, \mathrm{fb}}$ der kalten Phase, die effektive Kühlrate $\Lambda_{\text {eff }}$ der warmen Phase und dem Masseneintrag infolge von Kollisionen kalter Klumpen zusammen.

$$
\begin{aligned}
\dot{e}_{\mathrm{w}}= & {\left.\left[\left(1-\epsilon_{\mathrm{SN}}\right) e_{\mathrm{SN}}+A e_{\mathrm{c}}-(1+A) e_{\mathrm{w}}\right)\right] \frac{\dot{\rho}_{\mathrm{s}, \mathrm{fb}}}{\rho_{\mathrm{w}}} } \\
& -\left[f_{\mathrm{th}}+\left(1-f_{\mathrm{th}}\right) \epsilon_{\mathrm{tt}}\right] \Lambda_{\mathrm{eff}} \\
& -\epsilon_{\mathrm{CCC}}\left(e_{\mathrm{w}}-e_{\mathrm{c}}\right) \frac{\rho_{\mathrm{c}, \mathrm{pa}} r_{\mathrm{c}, \mathrm{coll}} l_{\mathrm{c}}^{3}}{\rho_{\mathrm{w}}}
\end{aligned}
$$

- Die Änderungsrate $\dot{K}$ der spezifischen turbulenten Energie des Gases ergibt sich aus der Zusammenstellung der Terme für den Eintrag durch SN, durch die thermische Instabilität sowie das externe Anregen $\Sigma$ von Turbulenz im Referenzvolumen und die turbulente Dissipation.

$$
\begin{aligned}
\dot{K}= & \left(\epsilon_{\mathrm{SN}} e_{\mathrm{SN}}-K\right) \frac{\dot{\rho}_{\mathrm{s}, \mathrm{fb}}}{\rho}+\left(1-f_{\mathrm{th}}\right) \epsilon_{\mathrm{tt}} \Lambda_{\mathrm{eff}} \frac{\rho_{\mathrm{w}}}{\rho} \\
& +\frac{\Sigma}{\rho}-C_{\epsilon} \frac{K^{3 / 2}}{l}
\end{aligned}
$$

- Die bereits oben gezeigte Gleichung 4.13 beschreibt die Änderung der Metallizität.

Die Beziehungen zwischen den verschiedenen Massen- und Energiereservoirs werden in Abb. 4.1 schematisch dargestellt.

\subsection{Gleichgewichtslösungen}

Neben Anderem habe ich quasi-stationäre Zustände des Modells unter Variation der primären Parameter untersucht. Da das Modell nicht analytisch lösbar ist, habe ich hierzu ein vom mir erstelltes Computerprogramm verwendet, um die Modellgleichungen numerisch zu integrieren. Als primäre Parameter gelten im Rahmen des Modells die Gesamtdichte des Gases $\rho$, die Metallizität $Z$ sowie die Injektionsrate $\Sigma$ turbulenter Energie aus externen Quellen, welche allesamt in diesem Kontext konstant gehalten werden. Dies bedeutet unter anderem, dass im Falle von Sternentstehung die Gesamtdichte immer wieder aufgefüllt werden muss, um einen QuasiGleichgewichtszustand zu erhalten. Als Maß für $\Sigma$ wird im Folgenden die Mach-Zahl $\mathcal{M}_{\Sigma}$ verwendet, die angibt, welche Turbulenzstärke sich in einem Gas bei konstant gehaltener Temperatur $T=T_{\mathrm{TI}}$ im Gleichgewicht zwischen konstantem $\Sigma$ und turbulenter Dissipation einstellte. 

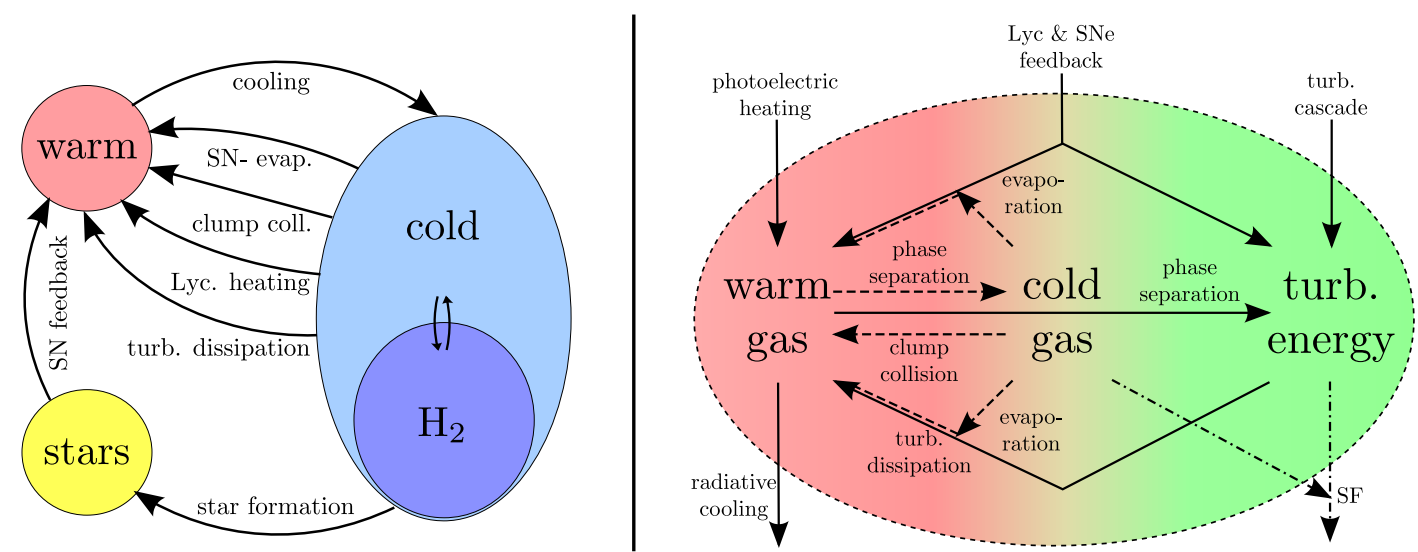

Abbildung 4.1: Links: Schematische Darstellung des Massenaustausches zwischen den verschiedenen Phasen des Modells in BS12. In roter, gelber bzw. blauer Farbe sind die Reservoirs der warmen Phase, der stellaren Komponente, bzw. der kalten Phase gezeichnet, wobei die kalte Phase zusätzlich eine molekulare Komponente, in dunklem Blau gefärbt, enthält. Massentransfer wird durch Pfeile angezeigt, die bestimmten Prozessen zugeordnet sind. Rechts: Schematische Darstellung des Energieaustausches zwischen den verschiedenen Reservoirs innerhalb des Gases inklusive externer Quellen und Senken. Die umschreibende Ellipse stellt den Energieinhalt des gesamten Gases dar, der in die thermische Energie der kalten Phase (blau), die der warmen Phase (rot) und die beiden gemeinsame turbulente Energie (grün) zerfällt. Auch hier zeigen Pfeile, benannt mit dem jeweils verantwortlichen physikalischen Prozess, Transfers an. Hierbei zeigen gestrichelte Pfeile Prozesse an, die mit dem Untermischen von kaltem Gas in warmes verbunden sind, während dies für die mit durchgezogenen Pfeilen dargestellten Prozessen nicht der Fall ist. Gestricheltgepunktete Pfeile beziehen sich auf den Verlust von Energie durch Umwandlung vom Gasmasse in Sternenmasse. (Übernommen aus BS12)
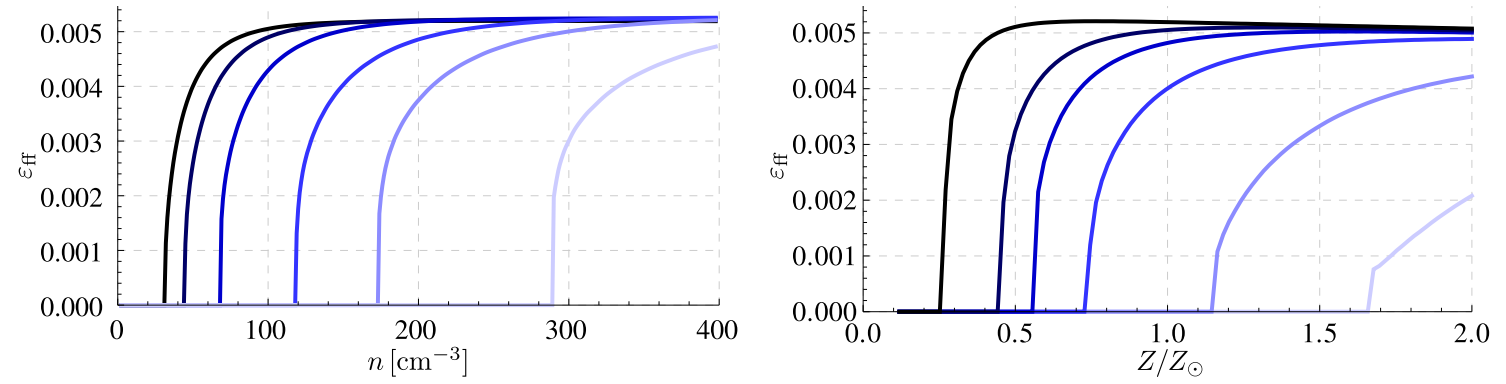

Abbildung 4.2: Links: Gleichgewichtssternentstehungseffizienz $\varepsilon_{\text {ff }}$ pro Freifallzeitskala über Gesamtteilchendichte $n$ für verschiedene Metallizitäten $Z / Z_{\odot}=$ 1.0, 0.8, 0.6, 0.4, 0.2 in Farben von dunklerem zu hellerem Blau. Rechts: Gleichgewichtssternentstehungseffizienz $\varepsilon_{\mathrm{ff}}$ pro Freifallzeitskala als Funktion von $Z / Z_{\odot}$ für verschiedene $n=200,100,75,50,25,12.5 \mathrm{~cm}^{-3}$ in Farben von dunklerem zu hellerem Blau. (Übernommen aus BS12) 

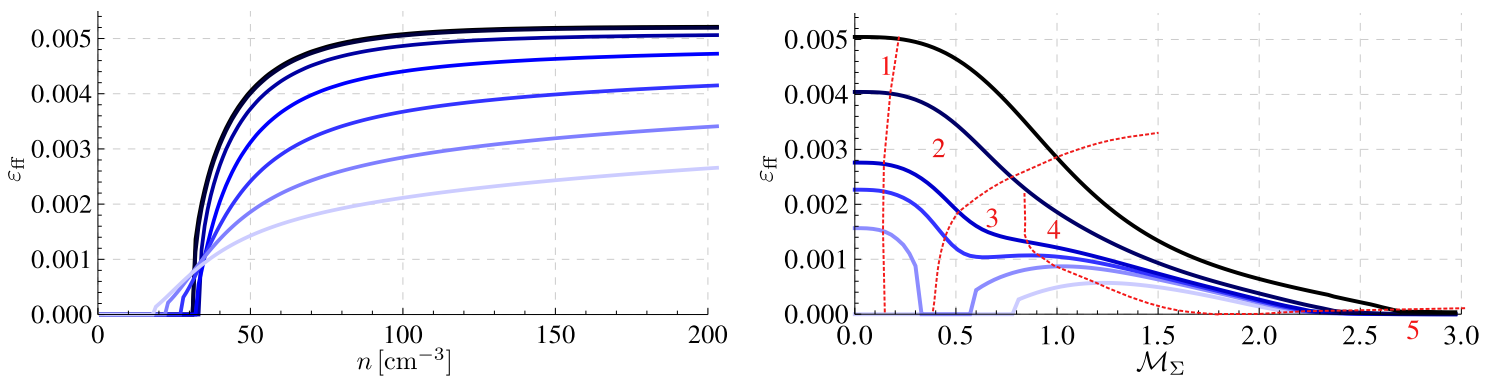

Abbildung 4.3: Links: Gleichgewichtssternentstehungseffizienz $\varepsilon_{\mathrm{ff}}$ pro Freifallzeitskala über Gesamtteilchendichte $n$ für verschiedene Injektionsraten $\Sigma$ turbulenter Energie ausgedrückt durch $\mathcal{M}_{\Sigma}=0.0,0.2,0.4,0.6,0.8,1.0,1.2$ in Farben von dunklerem zu hellerem Blau. Rechts: $\varepsilon_{\mathrm{ff}}$ als Funktion von $\mathcal{M}_{\Sigma}$ für verschiedene $n=27.25,32.5,35,37.5,50,100 \mathrm{~cm}^{-3}$ in Farben von dunklerem zu hellerem Blau. In rot ist die ungefähre Lage der Übergange zwischen den verschiedenen Regimes gekennzeichnet, die mit den roten Nummern identifiziert werden können (siehe Text). (Übernommen aus BS12 und Regimegrenzen hinzugefügt)

Bei Variation der Gesamtdichte $\rho$ lassen sich in den (Quasi-) Gleichgewichtslösungen drei verschiedene Regimes ausmachen, wie sie im linken Teil von Abb. 4.2 zu sehen sind.

- Bei zu geringen Dichten findet keine Sternentstehung statt. In diesem Fall sind keine Kaltphasenklumpen mit ausreichender Abschirmung vorhanden, unter deren Schutz molekulares Gas existieren würde. Die Existenz molekularen Gases, die im Modell als Hinweis auf die Abwesenheit von Heizprozessen, die gravitativem Kollaps entgegenwirken könnten, verstanden werden kann, ist jedoch Grundvoraussetzung für die Sternentstehung im Modell.

- Oberhalb einer Schwellendichte, ab der molekulares Gas existieren kann, steigt die Sternentstehungseffizienz steil an, um zu größeren Dichten hin wieder abzuflachen. Der Verlauf folgt in diesem mittleren Dichtebereich dem des Anteils molekularen Gases in der kalten Phase. Interessanterweise existieren in diesem Bereich teilweise zwei mögliche, stabile Lösungen, eine mit Sternentstehung und eine ohne. Nur die sternbildende Variante ist im linken Teil von Abb. 4.2 gezeigt. Welchen Zustand das System annimmt, hängt stark von den Anfangsbedingungen ab. Geben die Anfangsbedingungen keine aktive oder eine $\mathrm{zu}$ schwach aktive Sternentstehungsgeschichte vor, so wird das Modell aus Mangel an Heizquellen in den inaktiven Zustand verfallen. Mit zunehmender Dichte werden die Anforderungen an die Sternentstehungsvorgeschichte immer schwächer. Dieses sich aus den Modellgleichungen ergebende Zweiwegeverhalten ist Ausdruck des Umstandes, dass Sternentstehung über ihre mittelbaren Nach- 
wirkungen auf das ISM weitere Sternentstehung in umgebendem aber bislang inaktivem Gas über Druckerhöhung in der warmen Phase auslösen kann.

- Für hohe Gasdichten zeigt das Modell ein Sättigungsverhalten, in dem die Sternentstehungseffizienz gegen einen konstanten Wert konvergiert. Grund hierfür ist, dass auch der Anteil des molekularen Gases in der kalten Phase absättigt. Die konstante Effizienz wird durch die interne Selbstregulation im modellierten ISM zwischen Sternentstehung und stellarer Rückwirkung bestimmt. In diesem Dichtebereich zeigt das Modell das Verhalten einer KS-Relation mit Exponentialkoeffizienten $\alpha_{\mathrm{KS}}=1,5$.

Ein Vergleich der beiden Auftragungen in Abb. 4.2 zeigt, dass eine Variation der Metallizität $Z$ bei konstanter Dichte zum qualitativ selben Verhalten führt wie die Variation der Dichte bei konstanter Metallizität. Dies ist durch die starke Abhängigkeit der Abschirmung vor dissoziativer Strahlung in der kalten Phase von der absoluten Menge an Metallen im Volumen bedingt. Somit ist das Verhalten bezüglich einer Änderung der Dichte eine direkte Folge des veränderten Metallgehalts des Volumens. Dies erklärt auch die Verschiebung der Übergänge zwischen den Dichteregimes zu größeren Dichten hin, wenn die Metallizität verringert wird.

Die turbulente Energie $K$ hat als eine der fundamentalen Verlaufsgrößen des Modells Auswirkungen auf fast alle berücksichtigten Prozesse oder wird selbst von ihnen verändert. Das Verhalten des Modells bezüglich unterschiedlicher externer Injektionsraten $\Sigma$ ist komplexer als das bezüglich der Gasdichte, welches sich im Wesentlichen auf einen einzigen Prozess - die Abschirmfähigkeit des Kaltphasengases gegen energetische Strahlung - zurückführen lässt. Im linken Teil von Abb. 4.3 ist zu erkennen, dass zunehmende Injektionsraten den oben beschriebenen Übergangsbereich zwischen Inaktivität und Sättigung verbreitern. Besonders augenfällig ist die starke Verflachung des Absättigungsverhaltens mit zunehmendem $\Sigma$ bei $\mathcal{M}_{\Sigma}>0.4$. Auch die Minimaldichte für Sternentstehung verändert sich. Zunächst, für $\mathcal{M}_{\Sigma} \lesssim 0.6$, steigt die Grenzdichte für Sternentstehung marginal an, darüber aber fällt sie signifikant ab. Anhand des rechten Teils von Abb. 4.3 lassen sich die für das Verhalten des Modells in den fünf Regimes dominanten Prozesse erläutern, die sich bei Steigerung von $\Sigma$ zeigen.

- Für kleine $\Sigma \lesssim 0,2$ bleibt das bereits oben beschriebene Verhalten erhalten, da die Injektionsrate turbulenter Energie zu klein bleibt, um die Selbstregulation der Turbulenz signifikant zu beeinflussen. Die internen Quellen für Turbulenz dominieren in diesem Bereich, der in Abb. 4.3 mit '1' gekennzeichnet ist, klar über $\Sigma$. Auch das resultierende $K$ bleibt fast auf dem selben Niveau.

- Oberhalb von $\Sigma \sim 0,2$ schließst sich ein Bereich ('2') an, in dem die Sternentstehungseffizienz mit steigendem $\Sigma$ rasch abnimmt. Hierfür verantwortlich ist 
ein Anstieg von $K$, während die innere Energie des Gases ungefähr denselben Wert annimmt. Dies führt wegen des angenommenen Gleichgewichts der effektiven Drücke der warmen und der kalten Phase zu einer Verringerung der Kaltphasendichte $\rho_{\mathrm{c}, \mathrm{pa}}-$ und damit zu einem geringeren Abschirmvermögen bei ebenfalls verringerten Produktionsraten für molekularen Wasserstoff. Bei kleinen Dichten knapp oberhalb der Grenzdichte für Gleichgewichtssternentstehung ausgehend von $\Sigma \gtrsim 0$ kann diese Verringerung von $\rho_{\mathrm{c}, \mathrm{pa}}$ sogar die Sternentstehung insgesamt zum Erliegen bringen.

- Das sich für größere $\Sigma$ anschließende Regime '3' ist durch ein Abflachen des Abfalls der Sternentstehung im Bereich hoher Dichten und sogar ein Ansteigen von $\varepsilon_{\mathrm{ff}}$ für kleinere Dichten gekennzeichnet, was zu einer Verringerung der Sternentstehungsmindestdichte auch weit über die Grenzdichte im Falle von $\Sigma=0$ führt. Die Ursachen hierfür sind, dass einerseits der effektive Druck beider Phasen für ausreichend große $\Sigma$ durch den turbulenten Beitrag dominiert ist und daher für beide Phasen näherungsweise gleich ist, sodass $\rho_{\mathrm{c}, \mathrm{pa}}$ nicht weiter fallen kann $\left(\rho_{c, p a}>\rho\right)$. Andererseits wird durch die erhöhte Turbulenzstärke effektiv die Produktionsrate von molekularem Wasserstoff - wie auch die Sternentstehungseffizienz $\epsilon_{\mathrm{PN}}$ in der kalten Phase - erhöht. Durch stärkere Stöße im kalten Gas wird stärker komprimiertes Gas häufiger, was über eine verbreiterte Wahrscheinlichkeitsverteilung der Dichten in die Modellberechnungen eingeht. Für beide Effekte ist die Größe $\ell_{c}$ der Kaltphasenklumpen von Bedeutung, auf die $K$ herunterskaliert wird. Da $\ell_{\mathrm{c}}$ als Jeanslänge $\lambda_{\mathrm{J}, \mathrm{c}}\left(\rho_{\mathrm{c}, \mathrm{pa}}\right)$ definiert ist, findet man im Grenzfall $\rho_{\mathrm{c}, \mathrm{pa}} \rightarrow \rho$ bei geringeren $\rho$ bereits stärkere Turbulenz in der kalten Phase als bei höheren Dichten aber gleichem $\mathcal{M}_{\Sigma}$. Also vollzieht sich der Übergang für kleinere $\rho$ bereits bei geringeren $\Sigma$, da die kritische Machzahl $\mathcal{M}_{\mathrm{c}}$ für diesen Übergang wegen der Herunterskalierung der turbulenten Energie von $\ell$ nach $\ell_{\mathrm{c}}=\lambda_{\mathrm{J}, \mathrm{c}}\left(\rho_{\mathrm{c}, \mathrm{pa}}\right) \propto \rho_{\mathrm{c}, \mathrm{pa}}^{-1 / 2} \sim \rho^{-1 / 2}$ bereits bei geringeren $K$ und damit auch bei geringeren $\mathcal{M}_{\Sigma}$ stattfinden kann.

- Bei weiter steigendem $\Sigma$ sinkt die Sternentstehungseffizienz $\varepsilon_{\mathrm{ff}}$. Sie geht in eine näherungsweise lineare Abhängigkeit von $\Sigma$ über, deren Steigung unabhängig von der Dichte ist ('4'). Ursächlich für das Abfallen hier ist, dass die Kaltphasensternentstehungseffizienz $\epsilon_{\mathrm{PN}}$ nachlässt. Anschaulich liegt dies daran, dass bei zunehmender Turbulenz in der kalten Phase die Ausdehnung der Verdichtungsregionen abnimmt. Dadurch kann die kritische Masse einer Bonnor-Ebert-Sphäre, deren Dichte als untere Integrationsgrenze im PN12 Modell verwendet wird, nur mit zunehmend hohen lokalen Dichten erreicht werden, wenn $\Sigma$ gesteigert wird. Bei Dichten $\rho$ weit über der Minimaldichte für Sternentstehung sind die Regimes '3' und '4' nicht voneinander zu unter- 
scheiden. Bei hohen Dichten geht das Regime '2' direkt in '4' über, denn eine Verbreiterung der Dichteverteilung kann bei ohnehin bereits vollständig molekularem Gas den molekularen Anteil nicht weiter erhöhen, was für das in Regime (3) beobachtete Verhalten verantwortlich ist.

- Je nach Dichte findet oberhalb einer bestimmten Injektionsrate gar keine Sternentstehung mehr statt ('5'). Dieses $\Sigma$ ist dann erreicht, wenn das lineare Abfallen von $\varepsilon_{\mathrm{ff}}$ schließlich den Wert $\varepsilon_{\mathrm{ff}}=0$ erreicht.

Wie sich herausstellte sind CCC sehr seltene Ereignisse. Die Kollisionsrate entspricht dabei maximal einem solchen Ereignis in einem Zeitraum von 10 Ma. Daher können die entsprechenden Terme in den Modellgleichungen vernachlässigt werden $\left(\epsilon_{\mathrm{CCC}}=\right.$ 0 ), weil die Behandlung des intermittenten Phänomens der CCC im Rahmen der Gleichgewichtslösungen als ein über die Zeit gemittelter Effekt nicht sinnvoll ist. Sie sind vielmehr als Teil der zeitlich und räumlich aufgelösten Dynamik in der in Kapitel 5 vorgestellten LES zu verstehen, und bedürfen dort keiner expliziten Modellierung.

\subsection{Vergleich mit Beobachtungen}

Nachdem ich das Verhalten des Modells auch bezüglich Variation aller Modellkoeffizienten gründlich erforscht hatte, wie in BS12 ausführlich dargelegt ist, habe ich, soweit möglich, Vergleiche der Gleichgewichtslösungen mit Beobachtungen angestellt, die in BS12 ausführlicher dargelegt sind (siehe Anhang A.1). Hierbei zeigte sich, dass zum einen der modellierte Übergang von atomarem zu molekularem Gas bei empirisch plausiblen Säulendichten stattfindet. Zum Anderen sind die gewonnenen Sternentstehungseffizienzen bei Wahl physikalisch plausibler und laut Beobachtungen vertretbarer Werte für die Modellkoeffizienten im Einklang mit Beobachtungen. Weiterhin werden in den Resultaten für die Gleichgewichtszustände KS-Relationen reproduziert. Dabei weist die Relation

$$
\dot{\rho}_{\mathrm{S}} \propto \rho^{\alpha_{\mathrm{KS}}}
$$

einen Exponenten $\alpha_{\mathrm{KS}}=1,5$ auf, während die entsprechende Relation für molekulares Gas einen beinahe linearen Zusammenhang zeigt (siehe Abbildungen 21 und 22 in BS12), wie er zum Beispiel von Bigiel u. a. (2011) beobachtet wurde. 


\section{Kapitel 5}

\section{LES von Spiralgalaxien}

In diesem Kapitel werden die von mir durchgeführten Simulationen von idealisierten, isolierten Spiralgalaxien vorgestellt, die als Basis einer Veröffentlichung (Braun u. a., 2014, fortan BS14) in der Fachzeitschrift MNRAS dienten (siehe Anhang A.2). Zunächst gehe ich auf den für die Simulationen verwendeten Code Nyx ${ }^{1}$ (Almgren u. a., 2013) und die numerischen Methoden mit besonderem Fokus auf jene von mir implementierten ein (Abschnitt 5.1). Nach den Anfangsbedingungen der Simulationen (Abschnitt 5.2) stelle ich zuletzt die wesentlichen Ergebnisse einer der Simulationen dar (Abschnitt 5.2.2) und gehe auf mögliche zukünftige Erweiterungen des auf dem zuvor (Kapitel 4) vorgestellten ISM-Modell (BS12) basierenden Feinstrukturmodells MIST ein.

\subsection{Numerische Methoden}

In diesem Abschnitt wird kurz der kosmologische Hydrodynamik-Code Nyx mit seinen wesentlichen Bestandteilen knapp ${ }^{2}$ vorgestellt (Abschnitt 5.1.1), in den ich die speziell für meine Simulationen benötigten Komponenten integrierte. Darauf folgt eine Vorstellung des SGS-Modells für hydrodynamische Turbulenz (Abschnitt 5.1.2). Insbesondere wird auf das verwendete Feinstrukturmodell (Abschitt 5.1.3) eingegangen, welchem ich den Namen MIST (Mehrphasenmodell des ISM mit Sternentstehung und Turbulenz) gab. MIST ging aus dem in Kapitel 4 beschriebenen Modell

\footnotetext{
${ }^{1}$ Der Name 'Nyx' ist kein Akronym. Nyx wurde nach der Gottheit Nyx $(\nu v \xi)$ aus der griechischen Mythologie benannt. Sie ist sowohl Verkörperung als auch Göttin der Nacht und der Dunkelheit. Sie soll neben anderen Urgottheiten, wie Gaia $(\gamma \alpha \iota \alpha)$ - die Erde oder Erdgöttin - direkt aus dem ursprünglichen Chaos geboren worden sein, als die Weltordnung - also die Welt, wie wir sie kennen -, die die Griechen mit dem Begriff Kosmos verbinden, entstand. Das griechische Wort $\nu v \xi$ bezeichnet gleichzeitig die Göttin, wie auch die Nacht als Phänomen.

${ }^{2}$ Einen Überblick über die meisten numerischen Techniken in der Astrophysik gibt zum Beispiel Bodenheimer u. a. (2006).
} 
(BS12) hervor und beinhaltet auch die Methoden speziell zur Behandlung stellarer Partikel.

\subsubsection{Nyx}

Zum Beginn meiner Arbeit benutzte ich zunächst den kosmologischen Hydrodynamik-Code ENZO (O’Shea u. a., 2004; Bryan u. a., 2014). Aus der Vielzahl verschiedener kosmologischer Hydrodynamik-Codes fiel die Wahl deswegen auf ENZO, weil sein Rechengitter aus zusammenhängenden Blöcken aufgebaut ist. Jeder dieser Blöcke beinhaltet eine Vielzahl von gleichartigen, kubischen Volumenelementen ${ }^{3}$. Dies ist vor Allem im Hinblick auf die Anwendung des SGS-Modells von Schmidt u. Federrath (2011, fortan SF11) von Vorteil. Eine Implementierung dieses Modells wäre in baumartig strukturierten Gittern, wie in RAMSES (Teyssier, 2002), sogenannten 'moving-mesh'-Codes ohne streng reguläre Gitter wie AREPO (Springel, 2010) oder gar partikelbasierten Codes wie GADGET (Springel u. a., 2001) zumindest kaum praktikabel, da entspechend der Formulierung dieses Modells eine feste Auflösungsskala vorteilhaft ist, was in ENZO zumindest blockweise erfüllt ist. Leider stellte sich aber heraus, dass der in ENZO verwandte Hydrolöser nicht robust und stabil genug war, um die Problemstellung einer isolierten Scheibengalaxie zu bewältigen. So wandte ich mich der Verwendung von Nyx (Almgren u.a., 2013) zu, da auch hier blockweise strukturierte Rechengitter genutzt werden. Abgesehen von der übersichtlicheren Programmstruktur verfügt Nyx über einen Hydrolöser, der den Anforderungen meiner Problemstellung besser gewachsen ist.

Nyx ist ein sogenannter Hybridcode, der sowohl die strömungsmechanischen EulerGleichungen (siehe Kapitel 2.1.4) von baryonischem Gas auf einem numerischen Rechengitter löst, als auch andere kollisionsfreie Komponenten - im Falle kosmologischer Simulationen dunkle Materie und Sterne - in Form eines N-Körpersystems von massebehafteten Partikeln berücksichtigt. Nyx basiert auf dem Softwarepaket BoxLib, das ebenfalls am Lawrence National Laboratory in Berkeley (USA) unterhalten und entwickelt wird.

\section{Hydrodynamik}

Die Gasdynamik wird in Nyx mittels eines Godunov-Verfahrens zur Zeitintegration der Feldgleichungen auf dem (adaptiven) Rechengitter berücksichtigt. Dass die hydrodynamischen, integralen Erhaltungsgrößen unter Verwendung dieses Verfahrens tatsächlich erhalten bleiben, wird schon allein durch seine Konstruktion sichergestellt. Es läuft schrittweise ab, wobei für jeden Zeitschritt, dessen Länge ein Bruchteil

\footnotetext{
${ }^{3}$ Dabei kann die Größe der Volumenelemente verschiedener Gitterblöcke unterschiedlich sein, wenn das Gitter adaptiv verfeinert wird (siehe Abschnitt über AMR weiter unten).
} 
der durch das Courant-Lewi-Strauss-Kriterium vorgegebenen kritischen Länge ist, der folgende Zyklus durchlaufen wird. Zunächst werden mittels einer konservativen Rekonstruktion zweiter Ordnung (PPM; piecewise parabolic method) der verschiedenen hydrodynamischen Felder aus den zellenzentrierten Stützdaten die Feldwerte an den jeweiligen Zellgrenzen ermittelt und dann die sich ergebenden Riemannprobleme an allen Grenzflächen und für alle Raumrichtungen simultan mittels eines approximativen Lösers (oder eines exakten, falls der erstere versagt) gelöst, um die Flüsse der hydrodynamischen Erhaltungsgrößen über die einzelnen Übergangsflächen zwischen den Zellen zu erhalten. Aus den Flüssen werden die entsprechenden Inkremente für die anschließende Aktualisierung des Rechengitters berechnet. Nyx verwendet also ein numerisches Verfahren, das als 'unsplit scheme' bezeichnet wird, also ohne getrenntes Lösen (directional splitting) der Riemannprobleme in den einzelnen Raumrichtungen auskommt. Die in meinen Simulationen effektiv gelösten Feldgleichungen werden im Abschnitt 5.1.3 vorgestellt.

\section{Gravitation}

Um die Eigengravitation der dynamischen Komponenten innerhalb einer Simulation zu berechnen, nutzt Nyx einen Poissonlöser um das Gravitationspotential zu ermitteln, woraus sich die Gravitationsbeschleunigung durch räumliche Gradientenbildung auf dem Rechengitter bestimmen lässt. Ausgehend von der Dichte $\rho_{\text {dyn }}$ des dynamischen Anteils der schweren Masse in einer Simulation wird die Poissongleichung

$$
\triangle \Psi=4 \pi G \rho_{d y n}
$$

mittels des Gauss-Seidel-Rot-Schwarz-Relaxationsverfahrens iterativ im Rahmen eines Mehrgitteralgorithmus (multi grid solver) gelöst.

In meinen IDG-Simulationen wird die Eigengravitation der Gasmasse sowie der stellaren Masse auf diese Weise berücksichtigt. Die Konfiguration der dunklen Materie der IDG wird als statisch angenommen. Die gravitative Wirkung des Halos dunkler Materie auf die dynamischen Komponenten wird durch ein von mir implementiertes 'externes' Beschleunigungsfeld modelliert, welches auf das interne Gravitationsbeschleunigungsfeld addiert wird.

Die Wirkung der Gravitationsbeschleunigung wird mittels eines Prediktor-Korrektor-Verfahrens auf das Gas übertragen.

\section{Partikel}

Neben Lösung der hydrodynamischen Feldgleichungen bietet Nyx die Möglichkeit auch kollisionsfreie Fluidkomponenten zu berücksichtigen, die mit sich selbst und auch mit dem Gas über die Gravitation in Wechselwirkung stehen können. Diese 
werden in Form eines N-Körpersystems (N-body) behandelt. Hierbei wird das kollisionsfreie Fluid durch eine Anzahl von massebehafteten Partikeln repräsentiert, die sich innerhalb des Simulationsvolumens gemäß Trägheit und Gravitationsbeschleunigung bewegen. Zur Berechnung der Gravitation werden die Partikelmassen auf das zugrunde liegende Rechengitter mithilfe eines 'cloud in cell' (CIC) Verfahrens projiziert, wodurch ein dem der Gasdichte vergleichbares Dichtefeld erzeugt wird. Die aus der Lösung der Poissongleichung abgeleiteten Gravitationsbeschleunigungen werden zur Anwendung auf ein Partikel von gittergestützten Werten an die jeweilige Partikelposition interpoliert. Dieses numerische Verfahren, Partikel über Gravitation mittels eines Rechengitters zu koppeln, wird als PM-Methode (particle mesh method) bezeichnet. Zur Propagation der Partikel kommt in Nyx ein sogenannter 'Kick-Drift-Kick'-Algorithmus - ein spezielles Prediktor-Korrektor-Verfahren - zur Anwendung. Mittels dieses Algorithmus werden die Bewegungsgleichungen der Partikel schrittweise zeitlich integriert. Innerhalb eines Zeitschrittes werden zuerst die Gravitationsbeschleunigungen an den jeweiligen Positionen der Partikel ausgehend von gitterbasierten Daten interpoliert. Mithilfe der Gravitationsbeschleunigungen werden die Partikelgeschwindigkeiten zunächst um einen halben Zeitschritt inkrementiert $(\dot{u}=u+g \Delta t / 2)$, um dann mittels der resultierenden Geschwindigkeiten die Partikelpositionen um einen ganzen Zeitschritt zu verschieben $(x=x+u \Delta t)$. Simultan dazu wird gegebenenfalls das Gas um $\Delta t$ propagiert. Zuletzt, nach Lösen der Gravitation für den neuen Zustand des gesamten simulierten Systems, werden die Partikelgeschwindigkeiten mit den neuen, ebenfalls auf die Positionen interpolierten Beschleunigungen um den verbleibenden halben Zeitschritt fortentwickelt.

In kosmologischen Simulationen wird dunkle Materie in Form von Partikeln berücksichtigt. In meinen Simulationen isolierter Scheibengalaxien jedoch nehme ich einen statischen Halo aus dunkler Materie an, sodass nur stellare Materie als dynamische, kollisionsfreie Komponente verbleibt, für deren Modellierung allerdings zusätzliche Programmroutinen erforderlich sind (siehe hierzu Abschnitt 5.1.3).

\section{AMR}

Da Nyx wie die numerischen Codes Maestro und Castro vom Basissoftwarepaket BoxLib abgeleitet ist, verfügt Nyx über adaptive Gitterverfeinerung (AMR; adaptive mesh refinement). Dies ermöglicht es, Simulationen mit hoher räumlicher Auflösung bei gleichzeitig relativ geringem Aufwand an Rechnerressourcen durchzuführen. Hierbei werden mittels vordefinierter Kriterien Bereiche eines Rechengitters ausgewählt, über denen nachfolgend Gitterblöcke eingefügt werden, die über eine meist um den Faktor $f_{\text {ref }}=2$ höhere numerische Aufösung verfügen. Diese werden mit vom gröberen Gitter interpolierten oder bereits vorhandenen feinen Daten gefüllt. Dieses Verfahren kann mehrfach angewendet werden, sodass eine Hierarchie von Gittern 
mit unterschiedlichen, immer feineren Auflösungen entsteht. Im Programmablauf von Nyx werden auf diese Weise in regelmäßigen Abständen nach einer festgelegten Anzahl von Zeitschritten immer wieder aktualisierte Gitterstrukturen erzeugt, um sich der Entwicklung der hydrodynamischen Felder anzupassen. Damit die Felder in den Gittern verschiedener Aufösungen konsistent bleiben, werden die Gitterbereiche, über denen feinere Gitter liegen, mit Daten gefüllt, die sich aus der räumlichen Mittelung der darüber liegenden feineren Daten ergeben, wobei gegebenenfalls auch die Dichten der Impulse, der inneren Energie und eventuell auch der turbulenten SGS-Energie angepasst werden. Des weiteren müssen die Felder in an verfeinerte Bereiche angrenzenden Zellen auf die verfeinerten Flüsse über die Verfeinerungsgrenze hinweg korrigiert werden. Anstatt einen globalen Zeitschritt zu verwenden, der typischerweise durch die feinsten Gitterzellen vorgegeben wird, ermöglicht diese Form von AMR, die als blockweise strukturiert bezeichnet wird, sogenanntes 'sub-cycling'. Dabei können grobe Gitter mit einem relativ langen Zeitschritt fortentwickelt werden, während feinere Gitter entsprechend ihres Verfeinerungsfaktors mehrere Zeitschrittzyklen durchlaufen, was zusätzlich den Rechenaufwand reduziert.

AMR ist besonders nützlich für Simulationen, in denen man abhängig von der Problemstellung einen großen Dynamikbereich räumlicher Skalen abdecken muss, aber die volle Auflösung nicht an allen Stellen benötigt wird, da einige Prozesse, für die möglichst hohe Auflösung notwendig ist, nur an wenigen Orten innerhalb des Simulationsvolumens relevant sind. Das spezielle Verfeinerungskriterium, das zur Anwendung kommt, muss also geeignet sein, die Bereiche zu erfassen, die für die simulierten Prozesse relevant sind. Im Falle der isolierten Scheibengalaxien habe ich ein Kriterium gewählt, das auf der Gasdichte beruht. Dieses stellt sicher, dass überall dort, wo eine Dichte von etwa 0,1 Teilchen pro $\mathrm{cm}^{3}$ überschritten wird, mit maximaler Auflösung gerechnet wird. Oberhalb dieser Dichte kann das ISM als das mit dem BS12-Modell beschriebene Mehrphasensystem angenommen werden, in dem auch Sternentstehung stattfinden kann.

\section{Zusätzliche Quellterme}

Abgesehen von der Behandlung rein adiabatischer Hydrodynamik und Gravitation auf dem Rechengitter bietet Nyx die Möglichkeit, weitere lokale physikalische Prozesse wie etwa radiatives Kühlen des Gases oder Sternentstehung zu modellieren. Diese werden als zusätzliche Quellen- und Senkenterme vor und nach dem Hydrozeitschritt auf Grundlage des Zustandes des Gases berechnet oder können als externe Quellen eingespeist werden. Diese Quellterme beeinflussen wiederum den Zustand des Gases, was mittels eines Prediktor-Korrektor-Verfahrens numerisch bewerkstelligt wird.

Auf diese Art werden in den IDG-Simulationen sowohl das Modell für nicht aufgelöste Turbulenz nach SF11 als auch mein Modell für das turbulente, sternbildende 
ISM eingebunden.

\subsubsection{SGS-Modell}

Direkte numerische Simulationen von Turbulenz in astrophysikalischen Systemen wie Galaxien sind nicht möglich, auch wenn man alles abgesehen von reiner Hydrodynamik und Gravitation außer Acht lässt. Für solche Simulationen wäre es nötig, statt der Euler-Gleichungen die Navier-Stokes-Gleichungen (siehe Kapitel 2.1.3) zu lösen, wobei die Längenskala, auf der die physikalische Viskosität des Gases wirksam ist - die Kolmogorov-Skala (siehe Kapitel 2.3.2) - in der Simulation aufgelöst werden müsste. Während die Abmessungen des modellierten Systems von etlichen Parsec, Kiloparsec oder gar Megaparsec abgedeckt werden sollen, müsste die Simulation auch den gesamten Dynamikumfang des strömenden physikalischen Systems besitzen. Es ist aufgrund beschränkter Leistungsfähigkeit von Rechenmaschinen auch zukünftig schwer vorstellbar, solche Simulationen jemals durchzuführen. Eine Lösung dieses Problems stellen LES dar. LES zeichnen sich dadurch aus, dass auf einem Gitter explizit nur die Euler-Gleichungen ohne Berücksichtigung einer physikalischen Viskosität gelöst werden. Der Effekte der nicht aufgelösten Turbulenz wird durch Nutzung effektiver Modelle Rechnung getragen. Dies eignet sich besonders für Problemstellungen, bei denen man sich mehr für die Entwicklung des simulierten Systems auf großen Skalen interessiert. Außerdem ist bei Anwendung von LES keine exakte Modellierung der bei der Dissipation der Bewegungsenergie in Wärme involvierten Prozesse notwendig.

Meist nutzt man für solche Simulationen eine allen Gitterverfahren zur Lösung der hydrodynamischen Gleichungen gemeine Eigenschaft, die Geschwindigkeitsvariationen nahe der Gitterauflösung ausschmiert. Dieses numerische Artefakt, das wegen der Diskretisierung von Raum und Zeit entsteht, wirkt ähnlich einer physikalischen Viskosität und wird daher als numerische Viskosität bezeichnet. Diese begrenzt die effektive Reynolds-Zahl einer Simulation. Solange man nur Strömungseigenschaften weit oberhalb der Aufösungsskala untersucht, werden die Resultate nicht signifikant durch die begrenzte Auflösung beeinflusst, wenn die thermische Energie angepasst wird, um die auf der Aufösungsskala durch die numerische Viskosität dissipierte kinetische Energie auszugleichen und so Energieerhaltung zu gewährleisten. Simulationen, die dieses Verfahren nutzen, werden - etwas euphemistisch - als implizite LES (ILES) bezeichnet. Leider tauchen in ILES einige Probleme durch eben diese Methode auf. Einerseits bewirkt die numerische Viskosität einen Stau in der turbulenten Kaskade, der zu verstärkten Geschwindigkeitsfluktuationen auf Skalen knapp oberhalb der Gitterauflösung führt. Dies wird als Flaschenhalseffekt (bottleneck effect) bezeichnet. Andererseits würde die Energie, die bereits an der Gitterskala von kinetischer effektiv in thermische umgewandelt wird, im physikalischen System erst 
später in Wärme dissipiert werden. Besonders in Simulationen, in denen auch innerhalb des Gases ablaufende Prozesse modelliert werden, die explizit von der Temperatur des Gases abhängig sind, wie zum Beispiel radiatives Kühlen oder chemische Prozesse, kann es zu teils erheblichen Problemen führen. Das Gas kann unter diesen Umständen zu schnell seine Energie verlieren und damit auch einen Teil seines effektiven Druckes, der es gegen gravitativen Kollaps stabilisierte (Schmidt, 2014).

In Nyx wurde, auch unter meiner Mithilfe, ein explizites Modell zur Behandlung von kompressibler Turbulenz auf nicht aufgelösten Skalen implementiert, das von SF11 vorgestellt wurde und auf einer früheren Studie von Schmidt u. a. (2006) basiert. Dieses dynamische Modell eignet sich für meine Zwecke hervorragend, da relativ einfach zusätzliche Turbulenzquellen, wie sie sich aus dem BS12-Modell ergeben, eingebunden werden können. Wie nachfolgend beschrieben handelt es sich hierbei nicht um ein Gleichgewichts- oder Smagorinski-Modell für die nicht aufgelöste Turbulenz, bei dem zu jedem Zeitpunkt ein Gleichgewicht zwischen Produktion und Dissipation von SGS-Turbulenz angenommen würde. Durch die Wahl eines dynamischen Modells kann auch Aufbau oder Zerfall von Turbulenz auf kleinen Skalen verfolgt werden, sollten die entsprechenden Zeitskalen durch die Simulation aufgelöst werden. Wie sich zeigt, ist genau dies in meinen IDG-Simulationen der Fall, denn die relevanten Zeitskalen für Aufbau beziehungsweise Zerfall von SGS-Turbulenz liegen meist im Bereich von einigen Ma (siehe Abschnitt 5.2.2 oder BS14), während ein numerischer Zeitschritt auf den feinsten Gittern nur etwa $10^{4}$ a misst. Daher würde die Verwendung eines Gleichgewichtsmodells nicht der Dynamik des simulierten Systems gerecht werden. Insbesondere würde sich die Gewährleistung lokaler Energieerhaltung gerade auch im Hinblick auf die Wirkung des turbulenten Druckes auf die aufgelösten Skalen oder die Dissipation der SGS-Turbulenz in Wärme, die gegebenenfalls nicht zur eigentlich verfügbaren Energie passen würde, schwierig gestalten.

\section{SF11 Modell}

Nimmt man ausgehend von den Eulergleichungen für das Kontinuum nach dem von Germano (1992) entwickelten Formalismus eine Skalenseparation bei der Längenskala $\Delta$ vor, die mit der Gitterskala identifiziert werden kann, erhält man modifizierte Eulergleichungen für den aufgelösten Teil. Im Falle der auf diese Weise gefilterten Impulsdichte ergibt sich ausgedrückt in mit dem Tiefpass-Filteroperator $\langle\cdot\rangle_{\Delta}$ auf 
Länge $\Delta$ gefilterten Größen ${ }^{4}$ die partielle Differenzialgleichung

$$
\frac{\partial}{\partial t}(\rho \mathbf{u})+\nabla \cdot(\rho \mathbf{u} \otimes \mathbf{u})=\rho(\mathbf{g}+\mathbf{f})-\nabla P+\nabla \boldsymbol{\tau}_{\mathrm{SGS}}
$$

wobei $\mathbf{u}$ die Geschwindigkeit, $\mathbf{g}$ die Gravitationsbeschleunigung und $\mathbf{f}$ Beschleunigung aufgrund externer mechanischer Kräfte bezeichnet. Neben dem Druckterm $\nabla P$ taucht ein weiterer Term $\nabla \boldsymbol{\tau}_{\mathrm{SGS}}$ ähnlicher Form auf. Dieser beschreibt die Auswirkungen der herausgefilterten kleinen Skalen auf die gefilterte Impulsdichte. $\boldsymbol{\tau}_{\text {SGS }}$ wird als turbulenter Spannungstensor bezeichnet, der definiert ist als Differenz zwischen dem ungefilterten und dem gefilterten Advektionsterm

$$
\boldsymbol{\tau}_{\mathrm{SGS}}=\rho \mathbf{u} \otimes \mathbf{u}-\left\langle\rho_{\infty} \mathbf{u}_{\infty} \otimes \mathbf{u}_{\infty}\right\rangle_{\Delta}
$$

Im Rahmen von LES sind ungefilterte Größen natürlich prinzipiell nicht zugänglich. Dies ist ein Schließungsproblem analog zu dem bei Herleitung der hydrodynamischen Gleichungen aus der Boltzmann-Gleichung (siehe Kapitel 2.1), das mithilfe vereinfachender Annahmen und durch die Konstruktion der thermischen Energie, des Druckes (als Vereinfachung des mechanischen Spannungstensors) und zusätzlichen Zustandsgleichungen behoben wird. Analog zur thermischen Energie wird die turbulente SGS-Energie $K_{\text {SGS }}$ definiert $^{5}$

$$
\rho K_{\mathrm{SGS}}:=-\frac{1}{2}\left(\rho|\mathbf{u}|^{2}+\left\langle\rho_{\infty} \mathbf{u}_{\infty} \otimes \mathbf{u}_{\infty}\right\rangle_{\Delta}\right)=-\frac{1}{2} \operatorname{Spur}\left(\boldsymbol{\tau}_{\mathrm{SGS}}\right) .
$$

Nun wird offenbar, dass die nicht-aufgelöste kinetische Energie über einen Druck $P_{\mathrm{SGS}}$ in den gefilterten Gleichungen auf das Fluid wirkt, sodass sich der effektive Druck $P_{\text {eff }}$ als Summe auffassen lässt

$$
P_{\text {eff }}=P+\frac{2}{3} \rho K_{\mathrm{SGS}}=(\gamma-1) \rho e-\frac{1}{3} \operatorname{Spur}\left(\boldsymbol{\tau}_{\mathrm{SGS}}\right) .
$$

$K_{\mathrm{SGS}}$ dient als ein zwischen (aufgelöste) kinetische und thermische Energie geschaltetes Reservoir. Im Rahmen von LES wird $K_{\text {SGS }}$ als eigenständige Größe aufgefasst, die ihrer eigenen partiellen Differentialgleichung ${ }^{6}$ gehorcht

$$
\frac{\partial}{\partial t}\left(\rho K_{\mathrm{SGS}}\right)+\nabla\left(\mathbf{u} K_{\mathrm{SGS}}\right)=\Pi_{\mathrm{X}}+\Pi_{\mathrm{SGS}}-\rho \epsilon_{\mathrm{SGS}}+\mathfrak{D} .
$$

In einer LES muss diese neben den Euler-Gleichungen gelöst werden. $\Pi_{S G S}$ ist der turbulente Energiefluss durch die Filterskala $\Delta$, welcher hier als Produktionsrate

\footnotetext{
${ }^{4} \mathrm{Im}$ Folgenden verwende ich eine andere Notation als die in Kapitel 2.1 eingeführte. Makroskopische, aber ungefilterte, physikalische Größen (beispielsweise ' $y$ '), die zuvor mit einem Balken $(\bar{y})$ geschrieben wurden, tragen nun das Subskript ' $\infty$ ' $\left(y_{\infty}\right)$. Mit dem Tiefpass-Filteroperator $\langle\cdot\rangle_{\Delta}$ gefilterte Größen $\left\langle y_{\infty}\right\rangle_{\Delta}$ werden nun mit $y$ bezeichnet.

${ }^{5} \mathrm{Im}$ Gegensatz zu SF11 bezeichnet hier $K_{\mathrm{SGS}}$ nicht die SGS-Energiedichte, sondern die spezifische SGS-Energie.

${ }^{6}$ unter Vernachlässigung einiger für meine Anwendung vernachlässigbarer Terme
} 
der SGS-Energie verstanden werden kann, $\rho \epsilon_{\mathrm{SGS}}$ ist die über die Filterskala gemittelte Dissipationsrate der SGS-Energie in Wärme und $\mathfrak{D}$ beschreibt Transporteffekte durch Turbulenz im nicht aufgelösten Bereich. $\Pi_{X}$ ist hier zunächst nur ein Platzhalter für andere Quellen oder Senken von SGS-Energie. Die Terme auf der Quellenseite von Gleichung (5.6) hängen eigentlich von Prozessen jenseits der Auflösung ab. SF11 schlagen approximative Schließungen dieser Terme im Falle von kompressibler Turbulenz vor, die sich ausschließlich aus numerisch aufgelösten Größen und $K_{\mathrm{SGS}}$ berechnen lassen.

- $\Pi_{\mathrm{SGS}}$ : Die Produktionsrate von SGS-Energie kann durch den turbulenten Spannungstensor $\boldsymbol{\tau}_{\text {SGS }}$ und Scherungstensor $\boldsymbol{S}$ beschrieben werden

$$
\Pi_{\mathrm{SGS}}=\sum_{i, j} \boldsymbol{\tau}_{\mathrm{SGS}, i j} S_{i j}
$$

Hierbei ist $\boldsymbol{S}$ definiert als der symmetrische Teil der Jakobi-Matrix der Geschwindigkeit, in Komponentenschreibweise ${ }^{7}$

$$
S_{i j}=\frac{1}{2}\left(u_{i, j}+u_{j, i}\right) .
$$

Für $\boldsymbol{\tau}_{\text {SGS }}$ schlagen SF11 die folgende Schließung vor, in Komponentenschreibweise:

$$
\begin{aligned}
\tau_{\mathrm{SGS}, i j}= & 2 C_{\tau 1} \Delta \rho\left(2 K_{\mathrm{SGS}}\right)^{1 / 2}\left(S_{i j}-\frac{1}{3} \sum_{k} u_{k, k} \delta_{i j}\right) \\
& -2 C_{\tau 2} \rho K_{\mathrm{SGS}} \frac{\sum_{k} u_{i, k} u_{j, k}}{\sum_{k, l} u_{k, l} u_{k, l}}-\frac{2}{3}\left(1-C_{\tau 2}\right) \rho K_{\mathrm{SGS}} \delta_{i j}
\end{aligned}
$$

mit den Schließungskoeffizienten $C_{\tau 1}=0,02$ und $C_{\tau 2}=0,75$, deren Werte von SF11 mittels numerischer Experimente ermittelt wurden, und dem KroneckerDelta $\delta_{i j}$. Der erste Term (mit Koeffizient $C_{\tau 1}$ ) für sich genommen entspricht hierbei der sogenannten eddy-viscosity closure (deutsch etwa: der Schließung durch turbulente Viskosität), die in inkompressiblen LES oft Verwendung findet. Dieser lineare Term dominiert im Falle schwacher Turbulenz, während der zweite, nicht-lineare Term $\left(\right.$ mit $\left.C_{\tau 2}\right)$ für stark ausgeprägte Turbulenz vorherrscht und auch negative Werte annehmen kann.

- $\rho \epsilon_{\mathrm{SGS}}$ : Unter der Annahme, dass die Effekte der physikalischen Viskosität auf der Filterskala vernachlässigbar sind, und dass der turbulente Energiefluss von größeren zu kleineren Skalen unterhalb der Filterskala im Mittel annähernd

\footnotetext{
${ }^{7} \mathrm{Im}$ Folgenden werden partielle räumliche Ableitungen der i-ten Komponente eines Vektors $\mathbf{X}$ nach der j-ten Raumrichtung mittels der Schreibweise $X_{\mathrm{i}, \mathrm{j}}:=\partial X_{i} / \partial x_{j}$ abgekürzt, wobei $\mathbf{x}$ ein Positionsvektor ist.
} 
konstant ist, ergibt sich aus dimensionalen Erwägungen heraus der folgende Ausdruck für die Dissipationsrate $\rho \epsilon_{\mathrm{SGS}}$

$$
\rho \epsilon_{\mathrm{SGS}}=C_{\epsilon} \frac{\rho K_{\mathrm{SGS}}^{3 / 2}}{\Delta}
$$

mit dem Dissipationskoeffizienten $C_{\epsilon}=1.58$.

- $\mathfrak{D}$ : Der Transportterm wird über einen Diffusionsansatz genähert, der auf dem Gradienten der spezifischen SGS-Energie aufbaut

$$
\mathfrak{D}=\nabla \cdot\left(\kappa_{\mathrm{SGS}} \nabla K_{\mathrm{SGS}}\right),
$$

wobei die turbulente SGS-Diffusivität mit $\kappa_{\mathrm{SGS}} \approx 0,65 \times \rho K_{\mathrm{SGS}}^{1 / 2}$ approximiert wird.

\section{SGS-Modell und AMR}

Bei der Verwendung von AMR in einer LES mit dem oben beschriebenen SGSModell ergeben sich Schwierigkeiten, da die turbulente SGS-Energie offensichtlich von der Auflösung $\Delta$ des jeweiligen Rechengitters abhängig ist.

Üblicherweise werden Daten einerseits von feineren Gittern auf das nächst gröbere durch Mittlung der Dichten über das Volumen der gröberen Volumenelemente projiziert, um eine Repräsentation des feinen Zustands in gröberen Gittern zu erhalten. Andererseits werden grobe Daten auf das nächst feinere Gitter mittels eines konservativen Verfahrens interpoliert, falls feinere Gitterblöcke eingefügt werden oder Randbedingungen für die Berechnung der Dynamik auf feineren Gittern benötigt werden. Dabei muss im Falle von ILES lediglich die thermische Energie angepasst werden, um diese konsistent zur Gesamtenergie und den Impulsen zu halten. Die Inkonsistenzen entstehen hier zwangsläufig durch das Abschneiden der numerischen Lösung bei zwei unterschiedlich großen Längenskalen. Bei konservativer Mittelung von einem feineren auf ein gröberes Rechengitter zum Beispiel gilt ${ }^{8}$

$$
\begin{array}{r}
\frac{1}{f_{\text {ref }}^{3}} \sum(\rho E)_{\text {fine }}=\frac{1}{f_{\text {ref }}^{3}} \sum\left[(\rho e)_{\text {fine }}+\frac{(\rho \mathbf{u})_{\text {fine }}^{2}}{2 \rho_{\text {fine }}}\right]=(\rho E)_{\mathrm{crse}} \\
\geq(\rho e)_{\mathrm{crse}}+\frac{(\rho \mathbf{u})_{\mathrm{crse}}^{2}}{2 \rho_{\mathrm{crse}}}=(\rho e)_{\mathrm{crse}}+\frac{\left[\sum(\rho \mathbf{u})_{\mathrm{fine}}\right]^{2}}{2 f_{\mathrm{ref}}^{3} \rho_{\mathrm{crse}}}
\end{array}
$$

wobei $f_{\text {ref }}=\Delta_{\text {crse }} / \Delta_{\text {fine }}$ der Verfeinerungsfaktor ist. Kleinskalige Geschwindigkeitsfluktuationen, die in der feineren Lösung sichtbar sind, sind bei gröberer Diskretisierung unter Umständen nicht mehr erkennbar, da sie weggemittelt wurden. Die

\footnotetext{
${ }^{8}$ Größen mit Subskript 'crse' beziehen sich auf ein grobes Rechengitter, solche mit 'fine' auf das nächst feinere.
} 
in ihnen steckende Energie taucht folglich auch nicht mehr als aufgelöste kinetische Energie auf, ist aber auch zunächst nicht in der thermischen Energie enthalten, was in ILES durch entsprechende Erhöhung der thermischen Energie behoben wird. Analoges gilt für die Interpolation auf feinere Rechengitter, nur dass hierbei die thermische Energie herabgesetzt werden muss.

Bei Verwendung eines expliziten SGS-Modells mit turbulenter SGS-Energie sollte konsequenterweise die Differenz zwischen den jeweiligen aufgelösten kinetischen Energien in einer groben Zelle und den feineren Zellen, die dasselbe Volumen abdecken, der SGS-Energie zugeschlagen werden. Das auf dieser Prämisse basierende Verfahren, die volle turbulente Energiekompensation (FTEC, full turbulent energy compensation), liefert gute Resultate in Simulationen, in denen großskalige, gerichtete Strömungsbewegungen abwesend sind. Eben diese dominieren jedoch in Simulationen, die auch Gravitation berücksichtigen, meist gegenüber ungerichteten turbulenten Bewegungen. Für solche Simulationen erwies sich die beschränkte turbulente Energiekompensation (CTEC, constrained turbulent energy compensation) als erfolgreich (siehe Schmidt u. a., 2014, im Folgenden SA14), und findet in meinen Galaxiensimulationen Anwendung.

Zunächst wird bei beiden Methoden die übliche konservative Mittlung beziehungsweise Interpolation vorgenommen und die Differenz $\Delta\left(\overline{\rho e_{k i n}}\right)$ zwischen den aufgelösten kinetischen Energien berechnet. Im Gegensatz zu FTEC wird bei CTEC wird der Betrag der Energiemenge $\Delta\left(\overline{\rho K_{\mathrm{SGS}}}\right)$, die beim Übergang zwischen zwei Verfeinerungsstufen der SGS-Energie entnommen oder hinzugeschlagen wird, durch $\Delta\left(\overline{\rho K_{\mathrm{SGS}}}\right)_{\max }$ begrenzt. Um abzuschätzen, welcher Anteil von $\Delta\left(\overline{\rho e_{k i n}}\right)$ maximal der turbulenten Kaskade zuzuschreiben ist, wird Kolmogorov's Geschwindigkeitsskalierungsgesetz $\left(\dot{u} \propto u \cdot(\hat{\ell} / \ell)^{\eta}\right.$ beim Übergang von Länge $\ell$ auf $\ell$ ) mit Skalierungskoeffizienten $\eta=1 / 3$ angewendet, im Falle der Interpolation feiner Daten

$$
\Delta\left(\overline{\rho K_{\mathrm{SGS}}}\right)_{\max }=\left(\rho K_{\mathrm{SGS}}\right)_{\mathrm{crse}}\left(1-f_{\mathrm{ref}}^{-2 \eta}\right)
$$

und bei Projektion auf grobe Gitter

$$
\Delta\left(\overline{\rho K_{\mathrm{SGS}}}\right)_{\max }=\left(\overline{\rho K_{\mathrm{SGS}}}\right)_{\mathrm{fine}}\left(1-f_{\mathrm{ref}}^{2 \eta}\right) .
$$

Wenn $\left|\Delta\left(\overline{\rho e_{k i n}}\right)\right|>\Delta\left(\overline{\rho K_{\mathrm{SGS}}}\right)_{\text {max }}$ gilt, wird der Überschuss der thermischen Energie zugeschlagen oder entnommen. Bei der Interpolation auf feinere Gitter werden die Energieinkremente proportional zum Resultat der rein konservativen Interpolation verteilt. Für weitere Einzelheiten zu FTEC, CTEC und deren Implementation inklusive der Behandlung von Grenzfällen möchte ich hier auf die Veröffentlichung SA14 verweisen, an deren Verfassung ich beteiligt war. 


\subsubsection{MIST}

MIST ist eine Anpassung des im vorigen Kapitel 4 beschriebenen Modells für das sternbildende, turbulente ISM (BS12) zur Verwendung in LES mit Kopplung an das SGS-Modell nach SF11 und SA14. Zu diesem Zweck werden zusätzlich zu den essentiellen hydrodynamischen Gittervariablen (und der SGS-Energie) sechs bezüglich des Hydrolösers passiv mitbewegte Gittervariablen eingeführt. Drei davon beschreiben die chemische Zusammensetzung des Gases, diese sind die Wasserstoffdichte $\rho_{\mathrm{H}}$, die Heliumdichte $\rho_{\mathrm{He}}$ und die Metalldichte $\rho_{\mathrm{Z}}$. Die übrigen drei sind erstens die partiale Kaltphasendichte $\rho_{\mathrm{c}}$, mit deren Hilfe der Mehrphasenzustand im Sinne des BS12 Modells festgelegt wird, zweitens die stellare Massendichte $\rho_{\mathrm{s}(\mathrm{m})},{ }^{9}$ die als Puffer zwischen Erzeugung stellarer Materie im Gitter und Erzeugung stellarer, kollisionsfreier Partikel dient und drittens die Partialdichte heißer SN-Ejekta $\rho_{\mathrm{h}}$. Die BS12Modellgleichungen werden für jedes Volumenelement als Ein-Zonen-Modell für den jeweiligen Hydrozeitschritt integriert, die sich ergebenden Veränderungen in Raten umgerechnet und zusammen mit den zum SGS-Modell nach SF11 gehörigen Raten an den Algorithmus zur Behandlung zusätzlicher Quellen übergeben. Ausführlicher wird MIST in der von mir abgefassten Veröffentlichung BS14 erläutert.

Die wichtigste Veränderung gegenüber dem BS12 Modell ist - neben der Einführung von $\rho_{\mathrm{h}}$ und der Auslagerung stellarer Masse ${ }^{10}$ an kollisionsfreie Partikel die Kopplung an das SGS-Modell, indem $K_{\text {SGS }}$ aus dem turbulenten SGS-Modell mit $K$ (bzw. $e_{\mathrm{t}}$ in BS12) identifiziert wird. Die dynamisch berechnete Rate $\Pi_{\mathrm{SGS}}$ wird als externe Produktionsrate $\Sigma$ turbulenter Energie aufgefasst. Die Produktion von SGS-Energie durch SN und thermische Instabilitäten (fortan mit $\Pi_{\mathrm{SN}}$ beziehungsweise $\Pi_{\mathrm{TI}}$ bezeichnet) des BS12-Modells werden als zusätzliche Quellen $\Pi_{\mathrm{X}}$ von $\rho K_{\mathrm{SGS}}$ (siehe Gleichung 5.6) interpetiert.

\section{Stellare Partikel und Supernova-Gasphase}

Im Rahmen der Entwicklung von MIST in jeder Zelle wird unter Anderem eine Sternentstehungsdichte $\dot{\rho}_{\mathrm{s}, \mathrm{SF}}$ berechnet. Entsprechend dieser Rate wird Masse aus dem Dichtefeld $\rho$ entnommen und einem passiv advektierten Feld $\rho_{\mathrm{s}(\mathrm{m})}$ zugeführt, das bei der Berechnung der Gravitation mit berücksichtigt wird und folgender Erhaltungsgleichung gehorcht

$$
\frac{\partial \rho_{\mathrm{s}(\mathrm{m})}}{\partial t}+\nabla \cdot\left(\rho_{\mathrm{s}(\mathrm{m})} \mathbf{u}\right)=\dot{\rho}_{\mathrm{s}, \mathrm{SF}}-\left.\frac{\partial \rho_{\mathrm{s}(\mathrm{m})}}{\partial t}\right|_{\mathrm{SP}} .
$$

Dieses Feld dient als Puffer zwischen Sternentstehung $\dot{\rho}_{\mathrm{s}, \mathrm{SF}}$ und der Erzeugung kollisionsfreier stellarer Partikel, repräsentiert durch den letzten Term in Gleichung (5.15).

\footnotetext{
${ }^{9}$ Subskript '(m)' steht für 'mesh', zeigt also an, dass es sich um eine Gittervariable handelt

${ }^{10}$ Dies schließt die Berechnung der stellaren Rückwirkungen mit ein.
} 
Um die Zahl erzeugter Partikel nach Möglichkeit gering zu halten, wird erst dann ein Partikelpaar erzeugt, wenn sich in einer Zelle eine gewisse Mindestmasse $2 \cdot m_{\mathrm{p}, \min } \approx$ $40 \mathrm{M}_{\odot}$ an Sternen angesammelt hat. Stellare Partikel repräsentieren im Rahmen meiner Simulationen nicht einzelne Sterne sondern vielmehr sogenannte einfache Sternenpopulationen, die unter Annahme einer universellen stellaren Anfangsmassenverteilung (IMF) durch ihre Entstehungszeiten, Metallizitäten und Gesamtmassen voll charakterisiert sind. Das Partikelpaar übernimmt neben der Metallizität des Gases auch seine aufgelöste Geschwindigkeit, zu der beide Partikel noch eine zusätzliche, aber aus Gründen der Impulserhaltung in entgegengesetzte Richtungen weisende Geschwindigkeitskomponente erhalten, die die nicht aufgelösten Bewegungen der Gaswolken innerhalb der Zellen abbildet. Zusätzlich zu der anfänglichen Masse kann ein Partikel nach seiner Entstehung für einen Zeitraum von $2 \mathrm{Ma}-$ sofern es nicht die maximale Partikelmasse von $\sim 400 \mathrm{M}_{\odot}$ überschritten hat, die sicherstellt, dass alle stellaren Partikel von vergleichbarer Masse sind - alle in derselben Zelle wie es selbst befindliche stellare Masse aus $\rho_{\mathrm{s}(\mathrm{m})}$ entlang seines Weges aufnehmen, wobei alle dem Partikel zugeschriebenen Eigenschaften massegewichtet aktualisiert werden.

Bei der stellaren Rückwirkung auf das Gas werden dieselben Prozesse einbezogen wie bereits in BS12. Agertz u. a. (2013) beispielsweise nutzen ein ausgefeilteres Modell der stellaren Rückwirkung, was die berücksichtigten Rückwirkungsprozesse anbetrifft. Sie verwenden jedoch keine turbulente Rückwirkung, wie ich sie implementiert habe, da ihnen dieser Kanal in ihren ILES nicht zur Verfügung steht. Dass ein Teil der SN-Energie in meinem Modell der SGS-Energie zugeschlagen wird, entspricht dabei etwa dem sogenannten 'kinetic feedback' (zu deutsch etwa kinetische Rückwirkung), wie es beispielsweise Agertz u. a. (2013) nutzten. Hierbei wird aufgelöste kinetische Energie in das Gas um ein aktives, stellares Partikel herum injiziert, statt wie im Fall von MIST an der Position des Partikels selbst SGS-Energie zu injizieren.

Die Beiträge stellarer Rückwirkung werden für jedes Partikel individuell anhand der jeweiligen Eigenschaften berechnet und anschließend mittels eines CIC-Algorithmus auf das Rechengitter projiziert. Die kinetische Energie $\dot{\rho}_{\mathrm{s}, \mathrm{FB}}^{\mathrm{ex}}$, die dabei zwar auf das Gas übertragen, aber wegen der Summation der Impulse nicht als aufgelöste kinetische Energie auftaucht, wird der SGS-Energie zugeführt. Die Impulsübertragung von Sternen auf das Gas, die in meinem Modell geschieht, rührt ausschließlich von der Bewegung der stellaren Partikel her, ist also nicht mit dem oben erwähnten 'kinetic feedback' zu vergleichen. Um das sogenannte Überkühlen (over-cooling) des Gases zu umgehen, damit die durch SN eingetragene Energie über den resultierenden Druck eine Expansion des Gases bewirken oder zumindest einen Beitrag zur Stabilisierung gegen die Eigengravitation leisten kann, habe ich im MIST-Modell ei- 
ne zusätzliche dynamische Gittervariable eingeführt. Es ist die Partialdichte $\rho_{\mathrm{h}}$ des heißen SN-Gases, die der Erhaltungsgleichung

$$
\begin{aligned}
\frac{\partial \rho_{\mathrm{h}}}{\partial t}+\nabla \cdot\left(\rho_{\mathrm{h}} \mathbf{u}\right)+ & \min \left[\rho_{\mathrm{h}} \nabla \cdot \mathbf{u}, 0\right]= \\
& \left.\frac{\partial \rho_{\mathrm{s}}}{\partial t}\right|_{\mathrm{FB}}-\rho_{\mathrm{h}} \exp \left(-t / \tau_{\mathrm{h}}\right),
\end{aligned}
$$

genügt. Diese zusätzliche Gasphase lebt innerhalb der warmen Phase $\rho_{\mathrm{w}}$ und besitzt eine konstante spezifische thermische Energie $e_{\mathrm{h}} \approx 0,1 \times e_{\mathrm{SN}}$. Das heiße Gas geht durch allmähliches Untermischen in die reguläre warme Phase über, was durch einen exponentiellen Zerfall mit Zerfallszeitskala $\tau_{\mathrm{h}} \approx 1$ Ma modelliert wird. Der letzte Term auf der linken Seite von Gleichung (5.16) sorgt dafür, dass $\rho_{\mathrm{h}}$ bei Expansion absinkt, was adiabatischem Herunterkühlen entspricht, welches anders wegen der konstant gehaltenen thermischen Energie $e_{\mathrm{h}}$ nicht modelliert werden kann. Damit aber bei Kompression $\rho_{\mathrm{h}}$ nicht ansteigt, wird dieser Term nur berücksichtigt, falls $\nabla \cdot \mathbf{u}<0$.

In Kombination mit der turbulenten Rückwirkung reicht diese heiße SN-Gasphase aus, um das 'over-cooling' zu verhindern. In Simulationen ähnlicher Art wie meiner wird oft die gesamte radiative Gaskühlung für $\sim 10$ Ma nach Produktion von SN unterbunden (Stinson u. a., 2010; Agertz u. a., 2011), um dies zu erreichen. Da in einer Sternpopulation über einen Zeitraum von etwa 40 Ma SN vom Typ II stattfinden, ist so in einer Sternentstehungsregion die gesamte Kühlung für mindestens $\sim 40 \mathrm{Ma}$ abgeschaltet. Meine Antwort auf das 'over-cooling'-Problem ist im Gegensatz dazu eine physikalisch plausible Lösung.

Aus den beschriebenen Anpassungen des BS12-Modells ergibt sich das in Abb. 5.1 gezeigte Schema des Massenaustausches zwischen den Gasphasen und den stellaren Komponenten.

\section{Angepasste Strömungsgleichungen}

Der Satz von insgesamt zehn Erhaltungsgleichungen, die im Rahmen meiner LES von isolierten Scheibengalaxien in Nyx auf den adaptiven numerischen Rechengittern gelöst wurden, enthält modifizierte Eulergleichungen. Diese lauten inklusive der SGS- und MIST-Terme

$$
\frac{\partial \rho}{\partial t}+\nabla \cdot(\rho \mathbf{u})=-\dot{\rho}_{\mathrm{s}, \mathrm{SF}}+\dot{\rho}_{\mathrm{s}, \mathrm{FB}}
$$

für die Gesamtgasdichte $\rho$, wobei $\dot{\rho}_{\mathrm{s}, \mathrm{SF}}$ die Sternentstehungsrate und $\dot{\rho}_{\mathrm{s}, \mathrm{FB}}$ die SNAuswurfrate ist,

$$
\begin{array}{r}
\frac{\partial(\rho \mathbf{u})}{\partial t}+\nabla \cdot\left[\rho \mathbf{u u}+\left(p+\frac{2}{3} \rho K_{\mathrm{SGS}}\right)-\boldsymbol{\tau}_{\mathrm{SGS}}^{*}\right]= \\
\rho \mathbf{g}-\mathbf{u} \dot{\rho}_{\mathrm{s}, \mathrm{SF}}+\mathbf{u}_{\mathrm{s}, \mathrm{FB}} \dot{\rho}_{\mathrm{s}, \mathrm{FB}}
\end{array}
$$




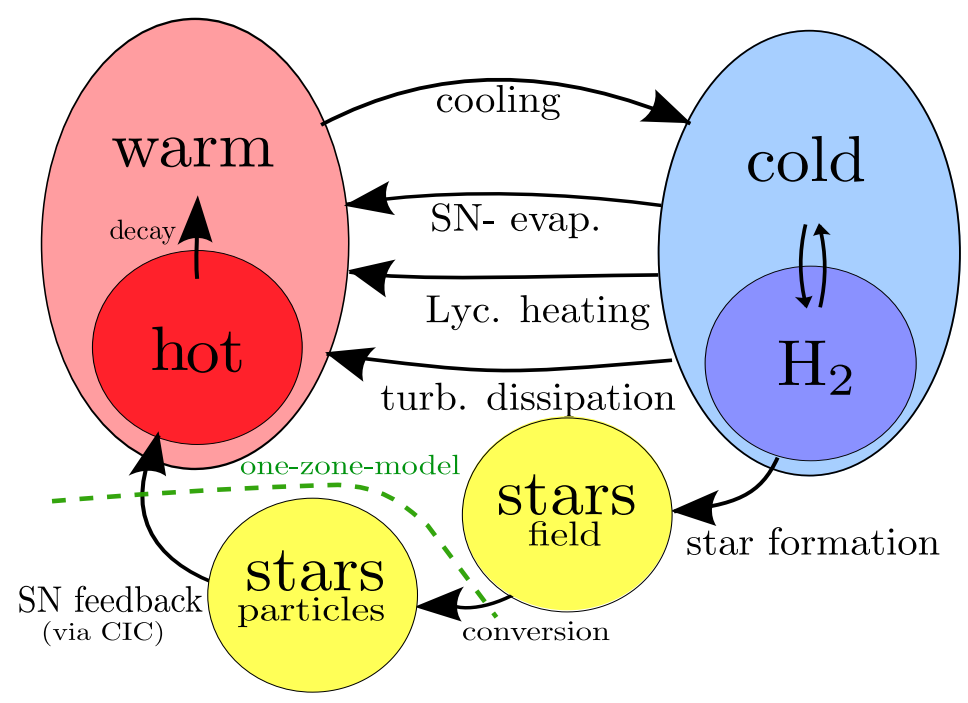

Abbildung 5.1: Schematische Darstellung des Massenaustausches zwischen den verschiedenen Gasphasen und Sternen in MIST. Arrangement ähnlich wie im linken Teil der Abb. 4.1, nur, dass die warme Phase durch ein helleres Rot und die zusätzliche heiße Phase in dunklerem Rot gehalten sind. Der oberhalb der gestrichelten grünen Linie befindliche Teil des Schemas wird im Rahmen des Ein-Zonen-Modells auf dem Rechengitter behandelt. Die stellare Komponente wurde in einen auf dem Rechengitter lebenden Anteil $\left(\rho_{\mathrm{s}(\mathrm{m})}\right)$ und einen in Form von Partikeln behandelten Teil der stellaren Masse aufgeteilt. 
für die Impulsdichte $\rho \mathbf{u}$, mit dem spurfreien SGS-Spannungstensor $\tau_{\mathrm{SGS}, i j}^{*}=\tau_{\mathrm{SGS}, i j}-$ $2 \delta_{i, j} K_{\mathrm{SGS}} / 3$ und der Geschwindigkeit der SN-Ejekta $\mathbf{u}_{\mathrm{s}, \mathrm{FB}}$, und

$$
\begin{array}{r}
\frac{\partial(\rho E)}{\partial t}+\nabla \cdot\left[\rho \mathbf{u} E+\left(p+\frac{2}{3} \rho K_{\mathrm{SGS}}\right) \mathbf{u}-\mathbf{u} \cdot \boldsymbol{\tau}_{\mathrm{SGS}}^{*}\right]= \\
\rho \mathbf{u} \cdot \mathbf{g}-\Lambda-\Pi_{\mathrm{TI}}-\Pi_{\mathrm{SGS}}-\left(E-e+e_{\mathrm{c}}\right) \dot{\rho}_{\mathrm{s}, \mathrm{SF}} \\
+\left(e_{\mathrm{SN}}+\frac{\mathbf{u}\left(2 \mathbf{u}_{\mathrm{s}, \mathrm{FB}}-\mathbf{u}\right)}{2}\right) \dot{\rho}_{\mathrm{s}, \mathrm{FB}}-\Pi_{\mathrm{SN}}
\end{array}
$$

für die Gesamtenergiedichte $\rho E=\rho e+1 / 2 \cdot \mathbf{u}^{2}$ des Gases exklusive der SGSEnergiedichte $\rho K_{\mathrm{SGS}}$. Hierbei bezeichnet $E$ die aufgelöste, spezifische Gesamtenergie des Gases, $e$ die Spezifische thermische Energie, $p=(\gamma-1) e$ den spezifischen thermischen Druck mit Polytropenexponent $\gamma=5 / 3, \Lambda=\rho \epsilon_{\mathrm{SGS}}+\Lambda_{\mathrm{c}}+\Lambda_{\mathrm{w}}$ die effektive Kühlrate zusammengesetzt aus der turbulenten Dissipationsrate und den jeweiligen radiativen Kühlraten der kalten und warmen Phase, $e_{\mathrm{c}}$ die konstante spezifische thermische Energie der kalten Phase und $e_{\mathrm{SN}}$ die thermische Energie der SN-Auswürfe. Die partielle Differenzialgleichung, die für die SGS-Energiedichte $\rho K_{\text {SGS }}$ gelöst wird, ist

$$
\begin{aligned}
& \frac{\partial\left(\rho K_{\mathrm{SGS}}\right)}{\partial t}+\nabla \cdot\left(\rho \mathbf{u} K_{\mathrm{SGS}}-\rho \kappa_{\mathrm{SGS}} \nabla K_{\mathrm{SGS}}\right)= \\
& \Pi_{\mathrm{SGS}}-\rho \epsilon_{\mathrm{SGS}}+\Pi_{\mathrm{TI}}-K \dot{\rho}_{\mathrm{s}, \mathrm{SF}}+\Pi_{\mathrm{SN}}+\dot{\rho}_{\mathrm{s}, \mathrm{FB}}^{\mathrm{ex}},
\end{aligned}
$$

wobei der Term $\dot{\rho}_{\mathrm{s}, \mathrm{FB}}^{\mathrm{ex}}$ die kinetische Energie der SN-Ejekta auffängt, die nicht in $\dot{\rho}_{\mathrm{s}, \mathrm{FB}} \mathbf{u}_{\mathrm{s}, \mathrm{FB}}^{2} / 2$ enthalten ist. Daneben werden auch die Gleichungen für die partialen Dichten $\rho_{\mathrm{X}}$ mit $\mathrm{X} \in\{\mathrm{H}, \mathrm{He}, \mathrm{Z}\}$, die die chemische Zusammensetzung des Gases festlegen, berücksichtigt.

$$
\frac{\partial \rho_{\mathrm{X}}}{\partial t}+\nabla \cdot\left(\rho_{\mathrm{X}} \mathbf{u}\right)=-\frac{\rho_{\mathrm{X}}}{\rho} \dot{\rho}_{\mathrm{s}, \mathrm{SF}}-\left.\frac{\partial \rho_{\mathrm{s}, \mathrm{X}}}{\partial t}\right|_{\mathrm{FB}},
$$

wobei der letzte Term die Injektionsrate der Partialdichte einer chemischen Komponente in den SN-Ejekta beschreibt. Im Rahmen des MIST-Modells ist zur Festlegung des Mehrphasenzustandes die Entwicklung einer Erhaltungsgleichung für die Kaltphasendichte notwendig

$$
\begin{aligned}
\frac{\partial \rho_{\mathrm{c}}}{\partial t}+\nabla \cdot\left(\rho_{\mathrm{c}} \mathbf{u}\right)= & \frac{\Lambda_{\mathrm{c}}+\Lambda_{\mathrm{w}} f_{\mathrm{TI}}}{e_{\mathrm{w}}-e_{\mathrm{c}}} \\
& -\dot{\rho}_{\mathrm{s}, \mathrm{SF}}-A_{\mathrm{SN}} \dot{\rho}_{\mathrm{s}, \mathrm{FB}} .
\end{aligned}
$$

$A_{\mathrm{SN}}$ und $f_{\mathrm{TI}}$ sind hierbei die aus dem BS12-Modell bereits bekannten Parameter für die Kaltphasenevaporation durch SN und die thermische Instabilität.

Die Entwicklungsgleichungen für SN-Gasdichte $\rho_{\mathrm{h}}$ und die stellare (Feld-) Dichte $\rho_{\mathrm{s}(\mathrm{m})}$ wurden bereits vorgestellt (Gleichung 5.15 beziehungsweise 5.16). 


\subsection{Simulationen}

In der Publikation BS14 werden die Ergebnisse von acht Simulationsläufen vorgestellt, mit deren Hilfe der Einfluss der numerischen Auflösung, des SGS- und MISTModells und der Implementierung der stellaren Rückwirkung auf die Entwicklung der simulierten Scheibengalaxie ausgelotet wurden. Hier beschränke ich mich auf die Ergebnisse des Referenzlaufes (in BS14 'ref' genannt), in dem SGS- und MISTModell voll zum Einsatz kamen.

\subsubsection{Anfangsbedingungen}

Innerhalb des periodischen Simulationskubus mit 0,5 Mpc Kantenlänge wurde mithilfe der von Wang u. a. (2010) entwickelten Potentialmethode eine isotherme Gasscheibe initialisiert. Diese Methode ermöglicht es, eine stabile vertikale Dichtestruktur in der Gasscheibe zu bestimmen. Dazu werden eine gegebene radiale Massenverteilung des Gases in der Scheibe, gegebene statische, radialsymmetrische Massenverteilungen anderer Komponenten wie der dunklen Materie oder einer bereits existierenden stellaren Scheibe sowie Annahme einer konstanten Temperatur $T_{\text {gas }}$ benötigt, um die Scheibenkonfiguration festzulegen. Dies ist möglich, da die vertikale Struktur der Scheibe nicht von der radialen Verteilung der Masse abhängig ist, wie Wang u. a. (2010) zeigen. Um die vertikale Dichtestruktur $\rho(R, z)$ der Scheibe im Abstand $R$ vom Zentrum, die durch

$$
\rho_{\mathrm{g}}(R, z)=\rho_{\mathrm{g}}(R, z=0) \exp \left(-\frac{\mu m_{\mathrm{H}} \Phi_{z}(R, z)}{k_{\mathrm{B}} T_{\text {gas }}}\right)
$$

beschrieben wird, zu berechnen, wird die vereinfachte Poisson-Gleichung

$$
\frac{\mathrm{d}^{2} \Phi_{\mathrm{g}, z}}{\mathrm{~d} z^{2}}=4 \pi G \rho_{\mathrm{g}}(R, z=0) \exp \left(-\frac{\mu m_{\mathrm{H}} \Phi_{z}(R, z)}{k_{\mathrm{B}} T_{\text {gas }}}\right)
$$

iterativ numerisch gelöst. $\Phi_{\mathrm{g}, z}=\Phi_{\mathrm{g}}(R, z)-\Phi_{\mathrm{g}}(R, z=0)$ ist das gravitative Potential des Gases allein, $\Phi_{z}(R, z)=\Phi_{\mathrm{g}, z}(R, z)+\Phi_{\mathrm{dm}, z}(R, z)+\ldots$ das alle massebehafteten Komponenten einbeziehende Gravitationspotential, $\mu m_{\mathrm{H}}$ die mittlere Partikelmasse des Gases und $k_{\mathrm{B}}$ die Boltzmann'sche Konstante. Mithilfe der bereits bekannten Potentiale $\Phi_{\mathrm{dm}, z}(R, z)+\ldots$ und der Randbedingung $\mathrm{d} \Phi_{\mathrm{g}, z}(R, z=0) / \mathrm{d} z=0$ kann Gleichung (5.24) integriert werden, wenn man zunächst einen Wert für $\rho(R, z=0)$ abschätzt. Der Schätzwert kann dann nach der Integration durch das neu berechnete $\Phi_{\mathrm{g}, z}$ angepasst werden (Gleichung 5.23), um den Vorgang der Integration und Anpassung des Schätzwertes sooft zu wiederholen, bis die gewünschte Genauigkeit der Lösung erreicht wurde, also $\rho_{\mathrm{g}}(R, z=0)$ ausreichend konvergiert ist. 
Die initiale Gasscheibe besitzt eine nach außen hin exponentiell (radiale Skala $r_{\text {gas }}=3.5 \mathrm{kpc}$ ) abfallende Oberflächendichte und eine Temperatur von $T_{\text {gas }}=$ $4 \times 10^{4} \mathrm{~K}$, aber keine stellare Komponente. Die Galaxienscheibe besteht anfänglich ausschließlich aus $10^{10} \mathrm{M}_{\odot}$ Gas, das eine Metallizität $Z=0,1 \cdot Z_{\odot}$ besitzt. Sie ist in einen statischen Halo dunkler Materie mit einem Dichteprofil wie von Navarro u. a. (1997, NFW) beschrieben eingebettet, der über das Beschleunigungsfeld

$$
\mathbf{g}_{\mathrm{dm}}=\frac{-G M_{\mathrm{dm}} \mathbf{r}}{\log \left(1+c_{\mathrm{dm}}\right)-\frac{c_{\mathrm{dm}}}{1+c_{\mathrm{dm}}}}\left(\frac{\log \left(r_{\mathrm{s}}\right)}{r^{3}}-\frac{c_{\mathrm{dm}}}{r_{\mathrm{dm}} r^{2} r_{\mathrm{s}}}\right)
$$

auf die dynamischen Komponenten der Simulation wirkt, wobei $\mathbf{r}$ ein Abstandsvektor ausgehend vom Galaxienzentrum ist, $r$ dessen Betrag und $r_{\mathrm{s}}=\left(1+r c_{\mathrm{dm}} / r_{\mathrm{dm}}\right)$ der dimensionslose skalierte Radius bei $\mathbf{r}$ ist. Das NFW-Haloprofil wird durch die Halomasse $M_{\mathrm{dm}}=10^{12} \mathrm{M}_{\odot}$, den Virialradius $r_{\mathrm{dm}}=213 \mathrm{kpc}$ und den Konzentrationsparameter $c_{\mathrm{dm}}=12$ vollständig fixiert. Diese Art der Initialisierung ist vorteilhaft, da die so erzeugte Galaxienscheibe zunächst adiabatisch stabil ist, wie vorab durchgeführte Testläufe ohne Kühlen und Sternentstehung bestätigten. In der Referenzsimulation wurden ausgehend von einem Grundgitter mit $256^{3}$ Zellen sechs Verfeinerungsstufen benutzt, sodass eine effektive Auflösung von 30 pc in den feinsten Gittern erreicht wurde.

Die Referenzsimulation wurde im Rahmen von BS14 bis zu einem simulierten Alter von 1 Ga entwickelt, was etwa 2,5 Umläufen des Gases in 10 kpc Abstand vom Galaxienzentrum entspricht.

\subsubsection{Ergebnisse}

\section{Entwicklung der Galaxie}

Die Entwicklung der Galaxienscheibe lässt sich anhand von Abb. 5.2 nachvollziehen. Nach Start der Simulation kollabiert die Scheibe wegen des radiativen Kühlens zu einer dünnen Scheibe. Diese ist anfällig für gravitative Instabilitäten (ToomreInstabilität), sodass sich zunächst vorübergehend spiralartige Strukturen und dann Klumpen bilden. In diesen Klumpen steigt die Dichte des metallarmen Gases so stark an, dass Sternentstehung einsetzt. Die in der Folge stattfindenden SNe sorgen dafür, dass die Gasklumpen wieder expandieren und so zerstört werden, wobei meist ein kleiner Sternhaufen zurückbleibt. Dieser stellt ein nur schwach gebundenes System dar, das mit der Zeit im Galaxienpotential zerrieben wird, falls es sich nicht zuvor mit anderen Sternhaufen verbindet und so massereiche Sternhaufen formt. Die meisten Sterne jedoch verlassen die Region ihrer Entstehung und bilden eine diffuse stellare Scheibe mit einer Geschwindigkeitsdispersion im Bereich von $\sim 10 \mathrm{~km} \mathrm{~s}^{-1}$. Die SNe bewirken die Entstehung von heißen Blasen, die aufgrund ihres Druckes das dichtere kühle Gas umherschieben und dabei teilweise auch verdichten. Das 


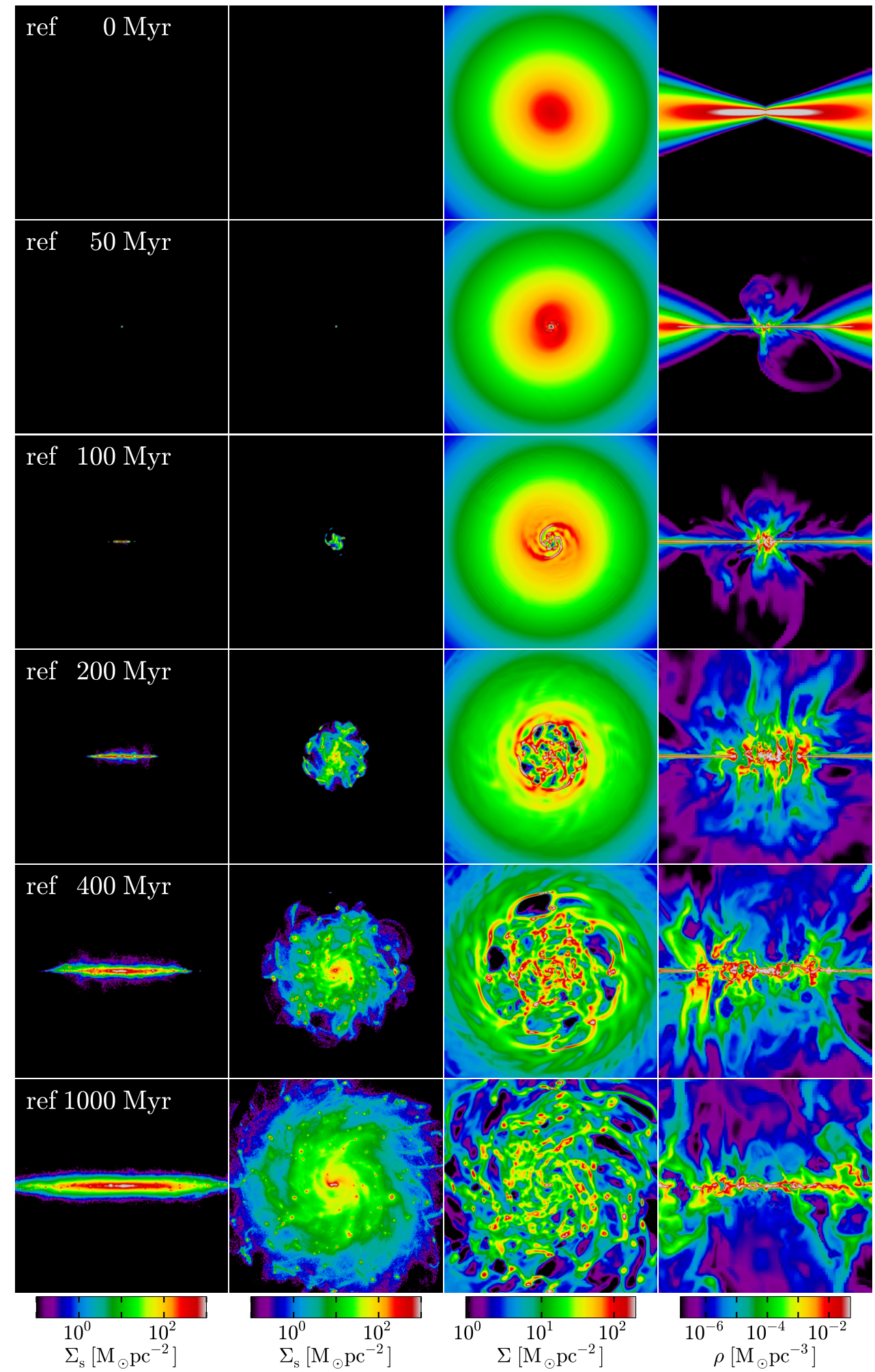

field of view : $20 \times 20 \mathrm{kpc}$

Abbildung 5.2: Zeitreihe der Galaxie im 'ref' Programmlauf zu ausgewählten Zeitpunkten von $0,50,100,200,400$ und $1000 \mathrm{Ma}$ (von oben nach unten): stellare Säulendichte $\Sigma_{\mathrm{s}}$ in äquatorialer oder polarer Richtung, Gassäulendichte $\Sigma$ in polarer Richtung Gasdichte und $\rho$ in äquatorialem Schnitt (von links nach rechts). 

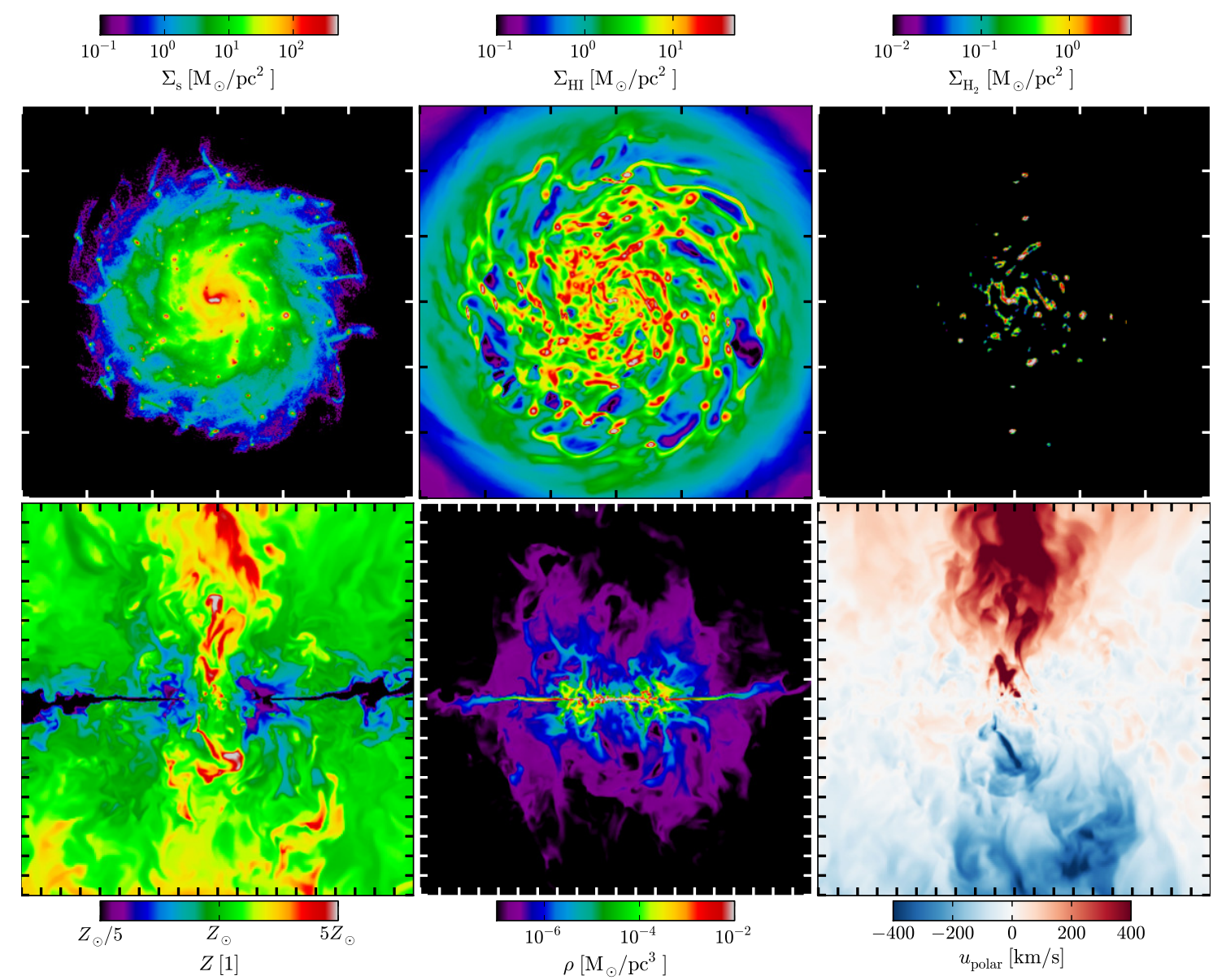

Abbildung 5.3: Oben: Drei Plots (von links nach rechts) der stellaren MassenSäulendichte $\Sigma_{\mathrm{s}}$, der neutralen Wasserstoffmassen-Säulendichte $\Sigma_{\mathrm{HI}}$ und der Sternentstehungs-Säulendichte $\dot{\Sigma}_{\mathrm{SF}}$ in der zentralen $(30 \mathrm{kpc} \times 30 \mathrm{kpc})$ Region der Galaxienscheibe nach 1 Ga. Unten: Polare Schnitte $(100 \mathrm{kpc} \times 100 \mathrm{kpc})$ durch den zentralen Bereich der Scheibe nach $1 \mathrm{Ga}$ (von links nach rechts): Metallizität $Z$, Gesamtgasdichte $\rho$ und Geschwindigkeit $v_{\text {polar }}$ senkrecht zur Scheibenebene. Die Markierungen am Rand der Plots haben jeweils einen Abstand von 5 kpc. (entnommen aus BS14) 
wiederum kann Sternentstehung auslösen. Die anfänglich ruhige und ebenmäßige Gasscheibe wird gestört und zeigt fortan eine löchrige, dynamische Struktur. Ausgehend von den SN-verursachten Löchern strömt heißes, metallangereichertes Gas nahezu senkrecht von der Scheibe mit Geschwindigkeiten von im Mittel $300 \mathrm{~km} \mathrm{~s}^{-1}$ - maximal bis zu $1000 \mathrm{~km} \mathrm{~s}^{-1}$ - ab, das auch kühleres, dichteres Material mit sich reißen kann. Diese SN-getriebenen Winde, die von den einzelnen heißen Blasen in der Gasscheibe ausgehen, vereinen sich ober- und unterhalb der Scheibe zu einem metallreichen, heißen und im Unterschallbereich turbulenten galaktischen Wind, der sich weit (mehrere hundert kpc) in die Umgebung der Galaxie ausbreitet. Mit steigender Metallizität in der Scheibe sinkt die Grenzdichte für Sternentstehung, sodass ein neuer Modus der Sternentstehung aufkommt, bei dem Sterne weniger lokalisiert gebildet werden. So findet, getrieben von SN- bedingten Verdichtungen, Sternentstehung wellenartig im bereits metallangereicherten inneren Teil der Scheibe statt. Noch später zeigt sich ein weiterer Sternentstehungsmodus. Bei diesem findet die Sternentstehung innerhalb massereicher Sternhaufen statt, die lokal das Schwerefeld dominieren. Während diese Sternhaufen aufgrund dynamischer Reibung langsam in das galaktische Zentrum hineinspiralisieren, können sie stets Gas aus der Scheibe akkretieren, welches dort entweder in Sterne umgewandelt oder als SN-getriebener Wind wieder abgeblasen wird.

Nach einer Milliarde Jahre Entwicklung und der Umwandlung von circa 30\% der anfänglichen Gasmasse in Sterne sieht die simulierte Scheibengalaxie aus wie in Abb. 5.3 gezeigt.

Im Verlaufe der Simulation ist die globale Sternentstehungsrate $\dot{M}_{\mathrm{s}}$ durch Selbstregulation zwischen Sternentstehung und stellarer Rückwirkung stets auf wenige Sonnenmassen pro Jahr begrenzt. Dabei steigt $\dot{M}_{\mathrm{s}}$, wie in Abb. 5.4 zu sehen, während der ersten $200 \mathrm{Ma}$ bis auf (zeitlich über circa 80 Ma gemittelte) $6 \mathrm{M}_{\odot} a^{-1}$ an, da der von Sternentstehung erfasste Bereich der Galaxie rasch anwächst. Danach flaut $\dot{M}_{\mathrm{s}}$ langsam wieder ab, was einerseits an den schrumpfenden Gasreserven in der Scheibe, andererseits an der bei gleicher Freifallzeit-Effizienz aber durch geringere Dichte im metallangereicherten, sternbildenden Gas verringerten Effektivität liegt. Auf Zeitskalen von wenigen $10 \mathrm{Ma}$ schwankt $\dot{M}_{\mathrm{s}}$ mitunter stark, wie ein Blick auf Abb. 5.4 verdeutlicht. Diese Schwankungen sind durch den Lebensweg einzelner Sternentstehungsgebiete bedingt, der von Beginn bis Einstellung der Sternentstehungaktivität 10 bis 30 Ma lang ist, was sich durchaus gut mit aus Beobachtungsdaten abgeleiteten Lebensdauern deckt (Blitz u. a., 2007; McKee u. Ostriker, 2007; Miura u. a., 2012). Aus $\dot{M}_{\mathrm{s}}$ und der verfügbaren Gasmasse $M_{\mathrm{g}}$ können zu jeder Zeit in der Simulation globale Sternentstehungszeitskalen $\tau_{\mathrm{SF}}=\dot{M}_{\mathrm{s}} / M_{\mathrm{g}}$ abgeleitet werden, auch diese sind gut mit Beobachtungsdaten vereinbar, wenn man junge, metallarme Galaxien zum Vergleich heranzieht (Daddi u. a., 2010; Genzel u. a., 2010). 


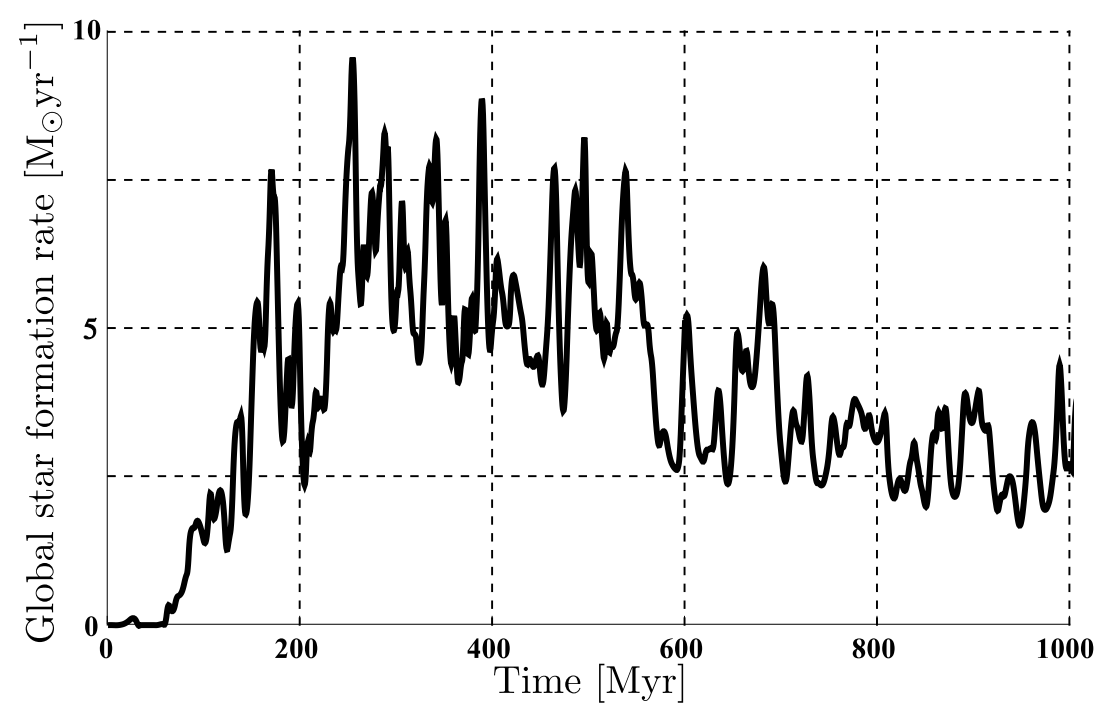

Abbildung 5.4: Globale Sternentstehungsrate $\dot{M}_{\mathrm{SF}}$ über Simulationszeit.

\section{Sternentstehungsrelationen}

Wie im rechten Teil von Abb. 5.5 leicht zu erkennen ist, besteht in meinen Simulationen eine enge, nahezu lineare Korrelation zwischen Sternentstehungssäulendichte $\dot{\Sigma}_{\mathrm{SF}}$ und der Säulendichte $\Sigma_{\mathrm{H}_{2}}$ molekularen Gases. Dieser fast lineare Zusammenhang ist nicht explizit in den Modellgleichungen angelegt, sondern ist ein Resultat der internen Selbstregulation des Modells, wie es sich bereits in BS12 zeigte, aber auch in Beobachtungen gefunden wurde (Gao u. Solomon, 2004; Lada u. a., 2010; Bigiel u. a., 2011). Allerdings ist die ermittelte Sternentstehungszeitskala bezüglich des molekularen Gases $\left(\tau_{\text {dep, } \mathrm{H}_{2}}=\dot{\Sigma}_{\mathrm{SF}} / \Sigma_{\mathrm{H}_{2}} \approx 80 \mathrm{Ma}\right)$ wesentlich kürzer als die von Bigiel u.a. (2011). Dies liegt zum Teil an den unterschiedlichen Auflösungen der Daten, hauptsächlich aber daran, dass die molekulare Dichte, wie sie in MIST verwendet wird, nicht die in Beobachtungen von Spektrallinien des Kohlenmonoxids gemessene abbildet. Es handelt sich im Falle von MIST um den dichtesten Teil des molekularen Gases, der aktiv beim Sternbildungsprozess beteiligt ist. Um ein mit Bigiel u.a. (2011) vergleichbares $\tau_{\mathrm{dep}, \mathrm{H}_{2}} \mathrm{zu}$ erhalten wäre es nötig, die chemische Entwicklung des Gases in den Phasen explizit zu modellieren. So ist mein $\tau_{\text {dep, } \mathrm{H}_{2}}$ in guter Übereinstimmung mit Beobachtungen von dichtem Gas in einzelnen Molekülwolken (Evans u. a., 2009; Murray, 2011).

Da mit MIST in seiner jetzigen Form die chemische Entwicklung des Gases nicht verfolgt wird, ist die weitere Analyse auf die Gesamtdichte neutralen Gases beschränkt, wobei all das Gas als neutral gilt, dessen Temperatur geringer als $T_{\text {neutral }}=$ $1,5 \times 10^{4} \mathrm{~K}$ ist, also $\rho_{\mathrm{HI}+\mathrm{H}_{2}}=\rho_{\mathrm{c}}+\rho_{\mathrm{w}, \mathrm{T}<\mathrm{T}_{\text {neutral }} .}$. Wie im rechten Teil von Abb. 5.5 ersichtlich, ergibt sich das übliche Verhalten der Sternentstehungsrate mit der Gas- 


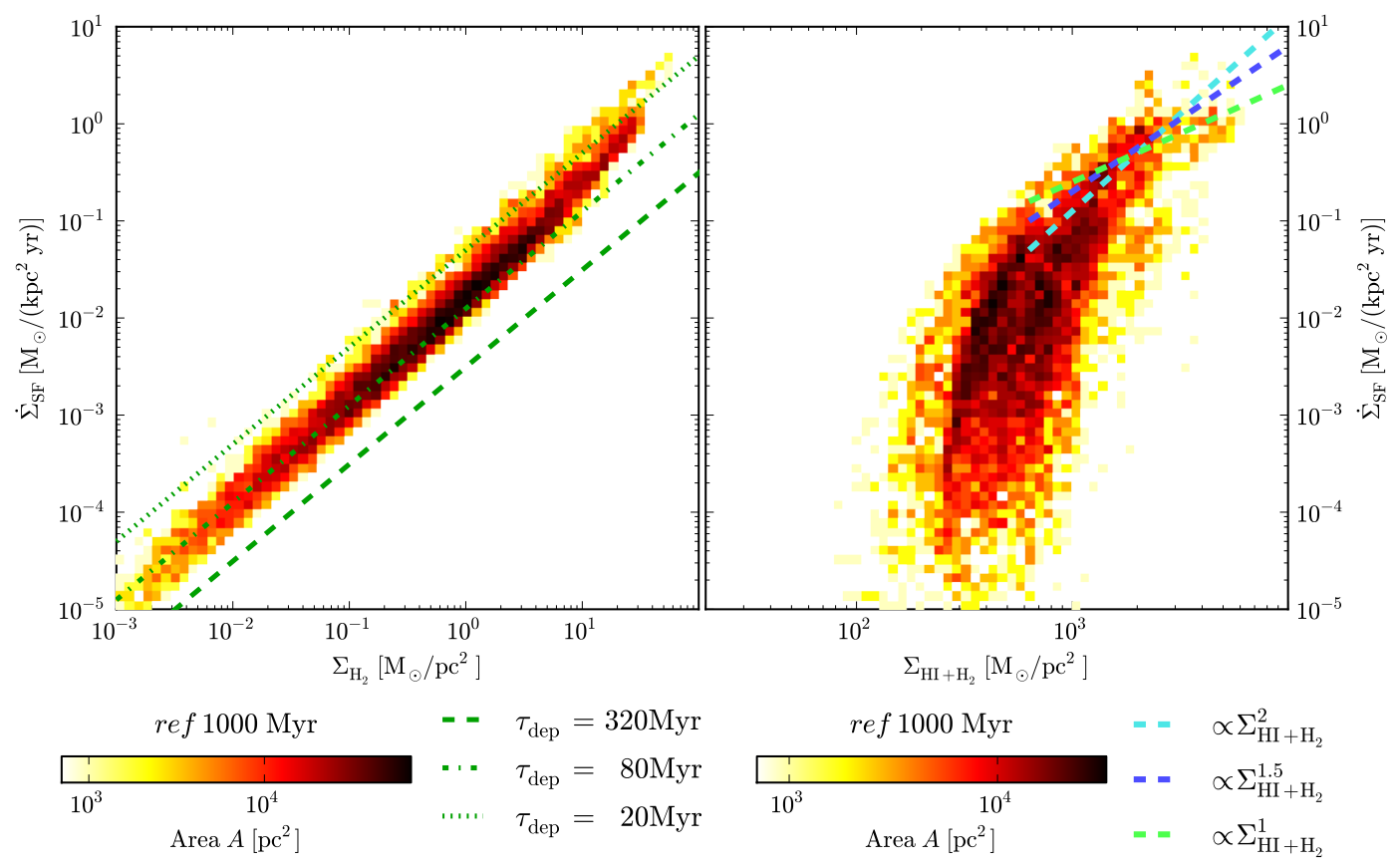

Abbildung 5.5: Rechts: Farbkodiertes zweidimensionales Histogramm bezüglich Sternentstehungssäulendichte $\dot{\Sigma}_{\mathrm{SF}}$ und Säulendichte $\Sigma_{\mathrm{H}_{2}}$ molekularen Gases. Grüne, unterschiedlich gestrichelte Linien zeigen konstante Sternentstehungszeitskalen (20, 80 und 320 Ma) an. Links: Farbkodiertes zweidimensionales Histogramm bezüglich Sternentstehungssäulendichte $\dot{\Sigma}_{\mathrm{SF}}$ und Säulendichte $\Sigma_{\mathrm{HI}+\mathrm{H}_{2}}$ neutralen Gases. Unterschiedlich gefärbte, gestrichelte Linien verdeutlichen den Verlauf von möglichen Sternentstehungsrelationen mit verschiedenen Potenzen $\left(\dot{\Sigma}_{\mathrm{SF}} \propto \Sigma_{\mathrm{HI}+\mathrm{H}_{2}}\right.$, $\dot{\Sigma}_{\mathrm{SF}} \propto \Sigma_{\mathrm{HI}+\mathrm{H}_{2}}^{1,5}$ und $\left.\dot{\Sigma}_{\mathrm{SF}} \propto \Sigma_{\mathrm{HI}+\mathrm{H}_{2}}^{2}\right)$.

dichte (vergleiche z.B. Bigiel u.a., 2011). Unterhalb einer gewissen Dichte (oder Säulendichte) findet keine Sternentstehung statt, darüber zeigt die Verteilung ein Absättigungsverhalten gegen eine KS-Relation $\left(\dot{\Sigma}_{\mathrm{SF}} \propto \Sigma_{\mathrm{HI}+\mathrm{H}_{2}}^{\alpha_{H I+H_{2}}}\right)$. Welchen Wert $\alpha_{H I+H_{2}}$ allerdings annimmt, lässt sich leider aus den Daten meiner Simulationen nicht abschließend klären, da der Schwanz der Verteilung zu hohen Säulendichten hin nicht gut genug ausgeprägt ist. Abgesehen davon ist bei einer räumlichen Auflösung von $\sim 30 \mathrm{pc}$ eine prominente KS-artige Relation auch nicht unbedingt zu erwarten (Onodera u. a., 2010). Die Verteilung der Daten in der $\Sigma_{\mathrm{HI}_{+} \mathrm{H}_{2}}-\dot{\Sigma}_{\mathrm{SF}}$-Ebene ist im Vergleich zu der von Bigiel u. a. (2011) gefundenen zu höheren Säulendichten verschoben. Die Gründe hierfür liegen in der geringeren Metallizität und dem hohen Gasanteil in der simulierten Galaxie relativ zu den beobachteten Galaxien in der erweiterten Nachbarschaft der Milchstraße. 


\section{Turbulenz}

Wie im rechten Teil der Abb. 5.6 zu sehen liegt die mittlere spezifische SGS-Energie in sternbildenden Regionen der simulierten Galaxie im Bereich von $100 \mathrm{~km}^{2} \mathrm{~s}^{-2}$, was einer Geschwindigkeitsdispersion von $\sim 10 \mathrm{~km} \mathrm{~s}^{-1}$ entspricht, wie sie auch in Beobachtungen vorgefunden wurde (siehe zum Beispiel Leroy u. a., 2008; Stilp u. a., 2013). Der recht enge Bereich von $10 \mathrm{~km}^{2} \mathrm{~s}^{-2} \lesssim K_{\mathrm{SGS}} \lesssim 10^{3} \mathrm{~km}^{2} \mathrm{~s}^{-2}$, in dem Sternentstehung stattfindet, ist auf der einen Seite begrenzt durch ein minimales Turbulenzlevel für die Existenz von abgeschirmtem, molekularem Gas, das für die Bildung von Sternen in MIST notwendig ist. Auf der anderen Seite begrenzt die stellare Rückwirkung und die durch sie bedingte Expansion und Verdünnung des Gases den $K_{\mathrm{SGS}}$-Sternentstehungsbereich. Eine typische sternbildende Region startet zunächst mit relativ geringer Turbulenz. Infolge weiteren gravitativen Kollapses und der Akkretion von weiterem Gas sowie durch Lyman-Kontinuums-Heizen steigt das Turbulenzniveau schnell an, bis es sich im Bereich von $K_{\mathrm{SGS}} \approx 100 \mathrm{~km}^{2} \mathrm{~s}^{-2}$ stabilisiert. In diesem Bereich verweilt eine sternbildende Region am längsten, weshalb dieser Bereich im $\epsilon_{\mathrm{ff}}-K_{\mathrm{SGS}}$-Diagramm am stärksten bevölkert ist. Mit Einsetzen der ersten SN-Explosionen wächst $K_{\mathrm{SGS}}$ zunächst langsam, wobei sich das Gas allmählich beginnt, sich aufgrund des gestiegenen (effektiven) Drucks auszudehnen. Mit der Zeit nimmt diese Expansion an Fahrt auf. Währenddessen sinkt die Gasdichte, wodurch Sternentstehung immer langsamer vor sich geht. Durch SN erzeugte SGS-Energie wird in immer dünneres Gas injiziert, was die Expansion noch weiter vorantreibt und schließlich lokal zum Ende der Sternentstehung führt.

Die Produktionszeitskala $\Pi_{\text {tot }} /\left(\rho K_{\mathrm{SGS}}\right)$ der SGS-Energie - wobei $\Pi_{\text {tot }}=\Pi_{\mathrm{SGS}}+$ $\Pi_{\mathrm{SN}}+\Pi_{\mathrm{TI}}-$ liegt im Mittel abhängig von $K_{\mathrm{SGS}}$ bei wenigen Ma bis zu $10 \mathrm{Ma}$, was auch in etwa der jeweiligen Dissipationszeitskala $\epsilon_{\mathrm{SGS}} / K_{\mathrm{SGS}}$ bei einem bestimmten Wert von $K_{\mathrm{SGS}}$ entspricht. So liegen die Zeitskalen bei $K_{\mathrm{SGS}} \sim 10 \mathrm{~km}^{2} \mathrm{~s}^{-2} \mathrm{im}$ Bereich von $10 \mathrm{Ma}$ und sinken bis $K_{\mathrm{SGS}} \sim 10^{3} \mathrm{~km}^{2} \mathrm{~s}^{-2}$ auf $\sim 1 \mathrm{Ma}$ ab. Produktion und Dissipation von SGS-Energie sind also näherungsweise im Gleichgewicht. Dies verdeutlicht auch die Darstellung von $\Pi_{\text {tot }} /\left(\rho \epsilon_{\mathrm{SGS}}\right)$ (grüne Linie) im linken Teil von Abb. 5.6. Im Rahmen von MIST besteht die Gelegenheit, die relative Wichtigkeit der verschiedenen Quellen der SGS-Turbulenz zu untersuchen, insbesondere, ob die SGS-Energieproduktion aufgrund von aufgelösten Strömungsbewegungen $\Pi_{\text {SGS }}$ oder durch interne Quellen $\Pi_{\mathrm{int}}=\Pi_{\mathrm{TI}}+\Pi_{\mathrm{SN}}$ überwiegt. Hier lassen sich im linken Teil von Abb. 5.6 fünf unterschiedliche Bereiche ausmachen.

- $K_{\mathrm{SGS}} \lesssim 1 \mathrm{~km}^{2} \mathrm{~s}^{-2}$ : In dem wenig bevölkerten Bereich liegt Material, das auf einen Gasklumpen oder Sternhaufen zufällt. Negative Werte von $\Pi_{\text {SGS }}$ zeigen expandierendes Gas an. Die relativ großen Werte von $\Pi_{\text {int }}$ werden durch die aktive Unterstützung eines minimalen $K_{\mathrm{SGS}}$ hervorgerufen, das für die Implementierung des SGS-Modells essenziell ist, da $\Pi_{\text {SGS }}$ direkt von $K_{\text {SGS }}$ abhängig 
ist (siehe Gleichungen 5.7 und 5.9).

- $1 \mathrm{~km}^{2} \mathrm{~s}^{-2} \lesssim K_{r m S G S} \lesssim 10 \mathrm{~km}^{2} \mathrm{~s}^{-2}$ : Hier überwiegt die Produktion die Dissipation leicht, insbesondere dominiert die Produktion durch die aufgelösten Gasbewegungen. Dies deutet auf Gas hin, das komprimiert wird, wobei mit zunehmendem $K_{\mathrm{SGS}} \Pi_{\mathrm{SGS}}$ immer stärker wird und $\Pi_{\text {int }}$, das in diesem Bereich hauptsächlich aus $\Pi_{\mathrm{TI}}$ gespeist wird, abnimmt.

- $10 \mathrm{~km}^{2} \mathrm{~s}^{-2} \lesssim K_{\mathrm{SGS}} \lesssim 10^{2} \mathrm{~km}^{2} \mathrm{~s}^{-2}$ : In diesem Regime setzt Sternentstehung ein, was dazu führt, dass mit zunehmendem $K_{\mathrm{SGS}} \Pi_{\mathrm{TI}}$ als führender Beitrag zu $\Pi_{\text {int }}$ allmählich durch $\Pi_{\mathrm{SN}}$ abgelöst wird und somit $\Pi_{\text {int }}$ wieder wächst. Weiterhin dominiert das schwächer werdende $\Pi_{\text {SGS }}$ die Produktion. Das Verhältnis von Produktions und Dissipationsrate nähert sich langsam dem Gleichgewicht an, was auf eine immer langsamere Kontraktion des sternbildenden Gases hindeutet.

- $10^{2} \mathrm{~km}^{2} \mathrm{~s}^{-2} \lesssim K_{\mathrm{SGS}} \lesssim 10^{3} \mathrm{~km}^{2} \mathrm{~s}^{-2}$ : Dies ist der Bereich, in dem sich die meiste Sternentstehung abspielt. Er ist gekennzeichnet durch die approximative Äquipartition zwischen $\Pi_{\text {tot }}$ und $\rho K_{\text {SGS }}$. $\Pi_{\text {SGS }}$ wird durch $\Pi_{\text {int }}$, das durch $\Pi_{\text {SN }}$ bedingt rasch zunimmt, als führender Betrag zu Produktion abgelöst. $\Pi_{\text {SGS }}$ wechselt sogar das Vorzeichen, was den Übergang von kontrahierenden zu expandierenden Umgebungen, aber auch die obere Grenze für Sternentstehung indiziert.

- $K_{\mathrm{SGS}} \gtrsim 10^{3} \mathrm{~km}^{2} \mathrm{~s}^{-2}$ : Gas, das sich in diesem Regime befindet, expandiert schnell, angetrieben von SN, was das negative $\Pi_{\mathrm{SGS}}$ anzeigt. Obwohl $\Pi_{\mathrm{SN}} \mathrm{zu}$ größeren $K_{\mathrm{SGS}}$ hin weiter zunimmt, kann diese hohe SGS-Energie nicht gehalten werden, da hier die Dissipation die Gesamtproduktion übersteigt. Deshalb findet sich auch kaum Gas mit $K_{\mathrm{SGS}}>10^{4} \mathrm{~km}^{2} \mathrm{~s}^{-2}$.

\subsubsection{Mögliche Erweiterungen von MIST}

Während ich mich bei der Konzeption von MIST auf die Struktur des ISM und die Auswirkungen von Turbulenz auf kleinen Skalen fokussiert habe, um die Entstehung von Sternen zu modellieren, bleibt die Modellierung der stellaren Rückwirkung auf die Effekte von SNe des Typs II und Lyman-Kontinuumsheizen beschränkt. Agertz u. a. (2013), zum Beispiel, haben eine elaboriertere Modellierung der stellaren Rückwirkung entwickelt, die auch die Effekte von stellaren Winden massereicher Sterne, SNe vom Typ Ia, stellaren Massenverlust massearmer Sterne in späten Entwicklungsphasen, sowie Strahlungsdruck ausgehend von heißen, massereichen Sternen einschließst. Diese Effekte könnten in einer zukünftigen Version von MIST ebenfalls 

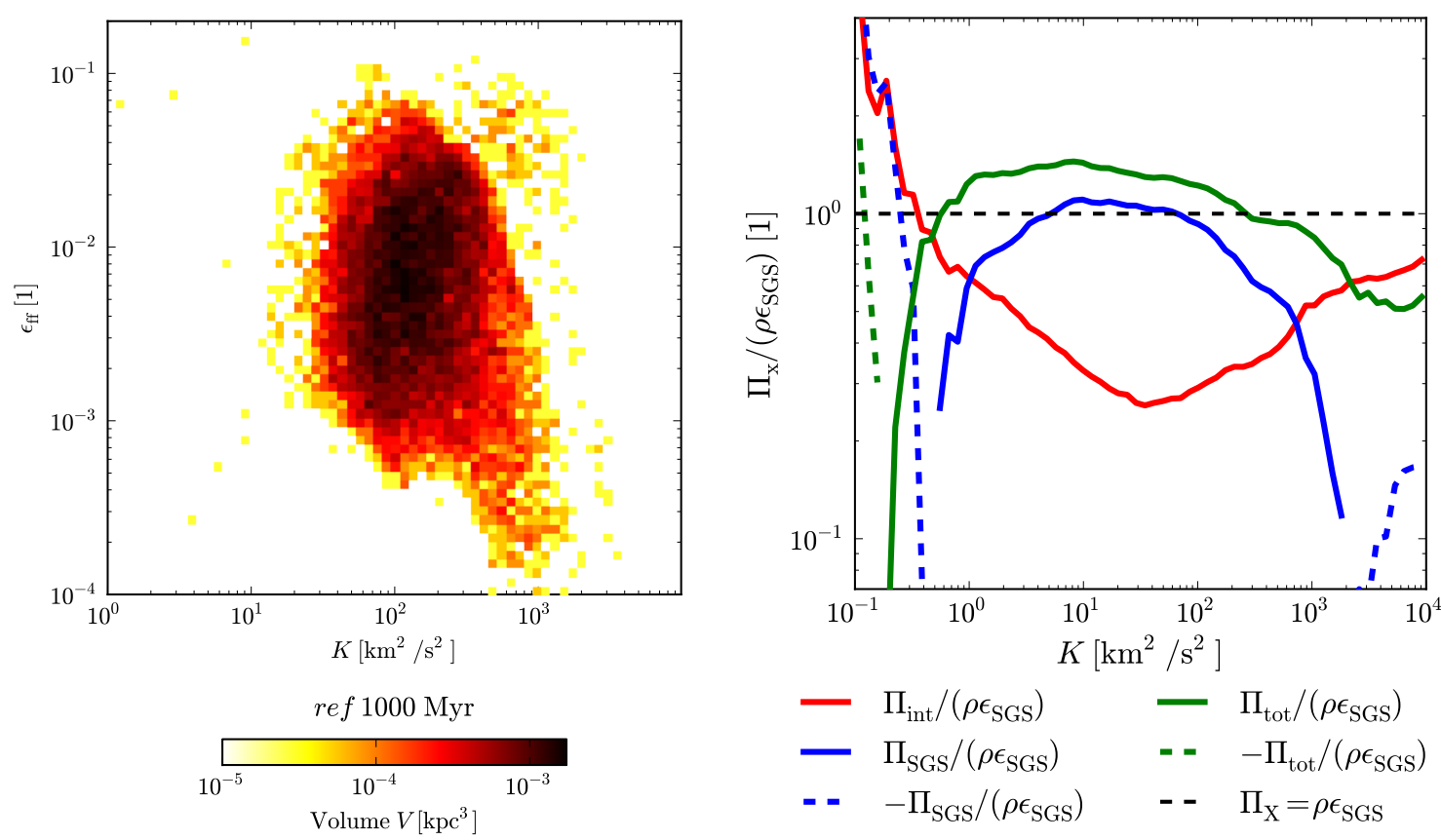

Abbildung 5.6: Rechts: Farbkodiertes zweidimensionales Histogramm bezüglich Sternentstehungseffizienz $\epsilon_{\mathrm{ff}}$ und spezifischer SGS-Energie $K_{\mathrm{SGS}}$. Links: Verhältnis $\Pi_{\mathrm{X}} /\left(\rho \epsilon_{\mathrm{SGS}}\right.$ von SGS-Energie-Produktion und -Dissipation über spezifischer SGSEnergie $K_{\mathrm{SGS}}$ : Rot $-\Pi_{\mathrm{int}} /\left(\rho \epsilon_{\mathrm{SGS}}\right)=\left(\Pi_{\mathrm{SN}}+\Pi_{\mathrm{TI}}\right) /\left(\rho K_{\mathrm{SGS}}\right)$, Blau $-\Pi_{\mathrm{SGS}} /\left(\rho \epsilon_{\mathrm{SGS}}\right)$, Grün $-\Pi_{\text {tot }} /\left(\rho \epsilon_{\mathrm{SGS}}\right)=\left(\Pi_{\text {tot }}+\Pi_{\text {tot }}\right) /\left(\rho \epsilon_{\mathrm{SGS}}\right)$. Gestrichelte Linien zeigen negative Werte einer Produktionsrate an. Äquipartition zwischen Produktion und Dissipation von SGS-Energie gilt entlang der gestrichelten schwarzen Linie. 
Berücksichtigung finden. So könnten insbesondere die Auswirkungen von stellaren Winden und SNe vom Typ Ia die Bildung extrem massereicher Sternhaufen unterdrücken. Stellare Winde erhöhten die Turbulenzstärke in sternbildenden Regionen bereits bevor sich SNe vom Typ II ereigneten, was weniger stark gebundene und damit kurzlebigere Sternhaufen zur Folge hätte. Durch SNe Ia stiege der effektive Druck des Gases in älteren Sternhaufen, was die Akkretion von weiterem Gas aus der Scheibe in den Sternhaufen erschwerte. Weiterhin wäre die explizite Modellierung der Wasserstoffchemie des Gases in den verschiedenen Phasen von MIST von Vorteil, da dies eine bessere Vergleichbarkeit zu Daten aus CO-Beobachtungen herstellte, und außerdem die Verwendung des Modells in Simulationen der Entstehung und Entwicklung von Galaxien im kosmologischen Umfeld erschlösse, da MIST derzeit nur in bereits mit Metallen leicht angereichertem Gas konzipiert ist.

An dieser Stelle möchte ich nicht unerwähnt lassen, dass Christoph Behrens unter meiner Mithilfe eine Studie zum Lyman- $\alpha$-Strahlungstransport in einer isolierten Scheibengalaxie basierend auf Daten des 'ref' Programmlaufs verfasst hat (Behrens u. Braun, 2014). 


\section{Kapitel 6}

\section{Einfluß des Sternentstehungsmodells}

In diesem Kapitel stelle ich eine Untersuchung des Einflusses des Sternentstehungsmodells der kalten Phase vor, die bislang noch nicht veröffentlicht wurde. Die Standardformulierung von MIST verwendet das effektive Sternentstehungsmodell $\left(\epsilon_{\mathrm{PN}}\right)$ von Padoan u. Nordlund (2011, fortan PN11) in der Parametrisierung mit einfachem Freifallzeitkoeffizienten von Federrath u. Klessen (2013, fortan FK13). Diese Wahl ist zunächst willkürlich, zumal weitere effektive Modelle zur Abschätzung der Sternentstehungsrate für turbulentes, kaltes Gas zur Verfügung stehen, die je nachdem, auf welchen Modellannahmen sie beruhen, unterschiedliche Abhängigkeiten vom turbulenten Zustand des Gases aufweisen. Die Struktur von MIST und die Anbindung an das SGS-Modell für Turbulenz von Schmidt u. Federrath (2011) bietet die einmalige Gelegenheit, solche Modelle in einer dynamischen Umgebung innerhalb einer Galaxiensimulation zu testen, da alle relevanten Zustandsinformationen lokal verfügbar sind. In dieser Studie habe ich die Modelle von PN11, Hennebelle u. Chabrier (2008, 2009, 2011, 2013, fortan HC08-13) und Krumholz u. McKee (2005, fortan KM05) sowohl in ihrer eigenen Formulierung sowie in derjenigen von FK13 - berücksichtigt, ebenso wie auch das von Padoan u. a. (2012, fortan PHN12) vorgeschlagene. Alle von mir untersuchten Modelle werden auch im kürzlich veröffentlichten Überblicksartikel Padoan u. a. (2013) behandelt.

Zunächst stelle ich diese Modelle hier kurz vor (Abschnitt 6.1), um dann auf die von mir durchgeführten Simulationsläufe einzugehen (Abschnitt 6.2) und deren Ergebnisse zu präsentieren (Abschnitt 6.3). Darauf folgen eine Diskussion dieser Ergebnisse (Abschnitt 6.4) und eine abschließende Zusammenfassung dieses Kapitels (Abschnitt 6.5).

\subsection{Sternentstehungsmodelle}

Abgesehen vom von PHN12 vorgestellten Modell können die analytisch hergeleiteten Sternentstehungsmodelle von PN11, HC08-09 und KM05 allesamt in einer Kategorie 
von Modellen zusammengefasst werden. Sie gehen davon aus, dass die Sternentstehungseffizienz $\epsilon_{\bmod }^{1}$ pro Freifallzeitskala $\tau_{\text {ff }}$, definiert als

$$
\tau_{\mathrm{ff}}(\rho)=\sqrt{\frac{3 \pi}{32 G \rho}}
$$

im Wesentlichen gegeben ist durch ein dichtegewichtetes - gegebenenfalls durch einen Freifallzeitfaktor $f_{\mathrm{ff} \text {,mod }}$ modifiziertes - Integral über die Wahrscheinlichkeitsdichteverteilung $\operatorname{pdf}(x)$ (mit dem logarithmischen Überdichteverhältnis $x=\log \left(\tilde{\rho} / \rho_{\mathrm{c}, \mathrm{pa}}\right)$ ) der lokalen Massendichte $\tilde{\rho}$ des Gases oberhalb einer kritischen Grenzdichte $\tilde{\rho}_{\text {crit,mod }}$. Bei der Definition von $\tilde{\rho}_{\text {crit,mod }}$ unterscheiden sich die einzelnen Modelle. Das kritische Überdichteverhältnis $\tilde{\rho}_{\text {crit,mod }} / \rho_{0}$ lässt sich hierbei durch verschiedene Kombinationen des Virialparameters einer Gaswolke $\alpha_{\text {vir }}$ (siehe Gleichung 4.9) und der turbulenten Mach-Zahl $\mathcal{M}$ der Bewegungen in ihrem Inneren

$$
\mathcal{M} \equiv \mathcal{M}_{\mathrm{c}}=\sqrt{\frac{2 K_{\mathrm{SGS}}\left(\ell_{\mathrm{c}} / \Delta\right)^{2 \eta}}{\gamma(\gamma-1) e_{\mathrm{c}}}}
$$

sowie modellspezifischer Koeffizienten ausdrücken, wobei hier bereits die Größen $\ell_{\mathrm{c}}$, $e_{\mathrm{c}}, K_{\mathrm{SGS}}, \Delta, \eta, \rho_{\mathrm{c}, \mathrm{pa}}$ und $\gamma$ eingesetzt wurden, die im Rahmen von MIST in den Simulationen verfügbar sind. Es wird angenommen, die pdf $(x)$ des kalten Gases sei der Form nach eine logarithmische Normalverteilung

$$
\operatorname{pdf}(x)=\frac{1}{\sqrt{2 \pi \sigma_{x}^{2}}} \exp \left(-\frac{\left(x-x_{0}\right)^{2}}{2 \sigma_{x}^{2}}\right)
$$

mit der mittleren Dichte $\rho_{0}=\rho_{\text {c,pa }}$ und der logarithmisch mittleren Dichte $x_{0}=$ $-\sigma_{x}^{2} / 2$. Die Standardabweichung $\sigma_{x}$ hängt unter Vernachlässigung von Effekten aufgrund magnetischer Felder maßgeblich vom turbulenten Zustand des Gases ab, hier ausgedrückt durch die turbulente Mach-Zahl $\mathcal{M}$ und den Faktor $b$, der die Art der turbulenten Mode parametrisiert (siehe Kapitel 4.1.2)

$$
\sigma_{x}^{2}=\log \left(1+b^{2} \mathcal{M}^{2}\right)
$$

Die Sternentstehungseffizienz $\epsilon_{\text {mod }}$ eines bestimmten Modells dieser Kategorie ergibt sich also zu

$$
\epsilon_{\text {mod }}=\frac{\epsilon_{\text {core }} f_{\text {cal,mod }}}{2} \int_{x_{\text {crit,mod }}}^{\infty} f_{\text {ff,mod }}(x) \exp (x) \operatorname{pdf}(x) \mathrm{d} x,
$$

wobei die Effizienz $\epsilon_{\text {core }}$ beschreibt, welcher Anteil der Masse in dichten, gravitativ gebundenen Gasklumpen (den sogenannten Kernen molekularer Wolken) schließlich in Sternen verbleibt. Hierbei ist $f_{\text {cal,mod }}$ ein modellspezifischer Kalibrationsfaktor.

\footnotetext{
${ }^{1}$ Das Subskript 'mod' dient als unspezifischer Platzhalter für die jeweiligen Sternentstehungsmodelle, deren Kürzel in Tabelle 6.1 aufgeführt sind.
} 
Für die Modelle dieser Familie wurden in FK13 modifizierte Versionen vorgestellt. Diese enthalten einen modifizierten Freifallzeit-Gewichtungsfaktor

$$
f_{\mathrm{ff}, \bmod }(x)=\frac{\tau_{\mathrm{ff}}\left(\rho_{\mathrm{c}, \mathrm{pa}}\right)}{\tau_{\mathrm{ff}}(\tilde{\rho})}=\exp \left(-\frac{x}{2}\right)
$$

(Multi-Freifallzeit, mff), wie er von HC08-13 vorgeschlagen wurde. Dadurch wird die Freifallzeit jeder individuellen Überdichte berücksichtigt. FK13 kalibrierten die Modellparameter der Modelle von HC08-13, KM05 und PN11 sowohl für die originale Form als auch für die mff-Variante anhand von Sternentstehungssimulationen in Boxen mit getriebener isothermer Turbulenz unter dem Einfluss der Eigengravitation des Gases. Da meine Simulationen keine magnetischen Felder berücksichtigen, verwende ich die Koeffizienten für den von FK13 'HD fit' genannten Fall in ihrer Tabelle 3 und als Wert für deren Parameter $\beta=\infty$.

Da FK13 in ihren Simulationen keine Massenauswürfe der 'prästellaren Partikel' berücksichtigten, wie er unter realen Bedingungen aufträte, muss die jeweilige Kaltphasensternentstehungseffizienz zur Verwendung in den Galaxiensimulationen mit einem zusätzlichen Vorfaktor $\epsilon_{\text {core }}$ berechnet werden. Dieser gibt an, welcher Anteil der anfänglichen Masse der Wolkenkerne schließlich in Sternen verbleibt.

Die in den Algorithmus von MIST integrierten Modelle sind mit Koeffizienten in Tabelle 6.1 aufgelistet.

\subsubsection{KM05-Modell}

Das kritische Überdichteverhältnis $\rho_{\text {crit,PN }} / \rho_{\text {c,pa }}$, das als untere Grenze des Integrals in Gleichung (6.5) dient, definieren KM05 als das mit einem Kalibrationsfaktor $\Phi_{x}^{2}$ modifiziertes Verhältnis der Quadrate der Jeanslänge $\lambda_{\mathrm{J}}\left(\rho_{\mathrm{c}, \mathrm{pa}}\right)$

$$
\lambda_{\mathrm{J}}\left(\rho_{\mathrm{c}, \mathrm{pa}}\right)=\sqrt{\frac{\pi \gamma(\gamma-1) e_{\mathrm{c}}}{G \rho_{\mathrm{c}, \mathrm{pa}}}}
$$

und der Längenskala $\lambda_{s}$

$$
\lambda_{s}=\ell_{\mathrm{c}} \frac{\gamma(\gamma-1) e_{\mathrm{c}} \Delta^{2 \eta}}{2 K_{\mathrm{SGS}} \ell_{\mathrm{c}}^{2 \eta}}
$$

unterhalb der die turbulenten Bewegungen des Gases aufgrund des Skalierungsverhaltens in der turbulenten Kaskade im Unterschallbereich liegen. Die Quadratur des Verhältnisses ist durch die Abhängigkeit $\lambda_{\mathrm{J}} \propto \rho_{\mathrm{c}, \mathrm{pa}}^{-1 / 2}$ bedingt. Diese Wahl von $\rho_{\text {crit,PN }} / \rho_{\text {c,pa }}$ ist motiviert durch die physikalische Überlegung, dass gravitativer Kollaps und damit Fragmentation, die schließlich in Sternentstehung gipfelt, erst möglich ist, wenn der Schwerkraft, die hier über $\lambda_{\mathrm{J}}$ eingeht, nur noch schwache Unterschallturbulenz als Ausgleich entgegensteht. Durch Zusammenfassung der Terme 
ergibt sich das von mir verwandte $\rho_{\text {crit,KM }} / \rho_{\mathrm{c}, \mathrm{pa}}$, welches für alle Modelle der KM05Familie unverändert bleibt, als

$$
\rho_{\text {crit }, \mathrm{KM}} / \rho_{\mathrm{c}, \mathrm{pa}}=\left(\Phi_{x} \frac{\lambda_{\mathrm{J}}\left(\rho_{\mathrm{c}, \mathrm{pa}}\right)}{\lambda_{s}}\right)^{2}=\frac{\pi^{2} \Phi_{x}^{2}}{5} \alpha_{\mathrm{vir}} \mathcal{M}_{\mathrm{c}}^{2}
$$

KM05 berücksichtigen in ihrem Modell keinen Freifallzeitgewichtungsfaktor. Also setze ich, wie bereits FK13, $f_{\mathrm{ff}, \mathrm{KM}}=1$ und $f_{\mathrm{ff}, \mathrm{KM}-\mathrm{FK}}=1$. Nur für das mff-Modell gilt $f_{\mathrm{ff}, \mathrm{KM}-\mathrm{FK}-\mathrm{mff}}^{2}=\rho / \rho_{\mathrm{c}, \mathrm{pa}}$. Die Kalibrationsfaktoren $\Phi_{x}$ und $f_{\text {cal,KM }}$ wurden von KM05 mittels Anpassung an Simulationsdaten von Vázquez-Semadeni u. a. (2003) $\mathrm{zu} \Phi_{x}=1,12$ und $f_{\mathrm{cal}, \mathrm{KM}}=1,92$ bestimmt. Für die anderen Modelle der KM05Familie verwende ich die von FK13 ermittelten Werte.

\subsubsection{HC08-13-Modell}

Das Modell von HC08-13 basiert auf dem Massenspektrum gravitativ gebundener Substrukturen innerhalb einer Gaswolke, die gemäß ihrer individuellen Freifallzeitskala kollabieren können. Die Sternentstehungseffizienz ergibt sich somit als mit dem mff-Faktor gewichtetes Massenintegral über dieses Massenspektrum bis hinauf zu einer Masse, bei der abgeschnitten wird um zu verhindern, dass zu große Teile einer Wolke, oder gar Substrukturen, die massereicher sind als die gesamte Wolke selbst, mit einbezogen werden. Dies lässt sich, wie von FK13 und Hennebelle u. Chabrier (2013) vorgeführt, durch Substitution in die Gestalt von Gleichung (6.5) überführen. Die kritische Überdichte definiert sich dann dadurch, dass die entsprechende turbulente Jeanslänge $\lambda_{\mathrm{J}, \mathrm{t}}\left(\rho_{\mathrm{cr}, \mathrm{HC}}\right)$ kleiner ist als ein gewisser Bruchteil $y_{\text {cut }}$ der Wolkengröße $\ell_{\mathrm{c}}$

$$
\lambda_{\mathrm{J}, \mathrm{t}}\left(\rho_{\mathrm{cr}, \mathrm{HC}}\right)=\left(\frac{\pi \gamma(\gamma-1) e_{\mathrm{c}}+2 \pi \lambda_{\mathrm{J}, \mathrm{t}}\left(\rho_{\mathrm{cr}, \mathrm{HC}}\right) K_{\mathrm{SGS}}\left(\frac{\ell_{\mathrm{c}}}{\Delta}\right)^{2 \eta}}{G \rho_{\mathrm{cr}, \mathrm{HC}}}\right)^{1 / 2}=y_{\mathrm{cut}} \ell_{\mathrm{c}} .
$$

Dies führt auf eine quadratische Gleichung für $\lambda_{\mathrm{J}, \mathrm{t}}$, die zwei Lösungen besitzt. Von diesen ist aber nur die mit der positiven Wurzel physikalisch sinnvoll, da bei Erhöhung des Druckes das Vermögen steigen muss, der Schwerkraft zu widerstehen

$$
\begin{aligned}
\lambda_{\mathrm{J}, \mathrm{t}}\left(\rho_{\mathrm{cr}, \mathrm{HC}}\right) & =\frac{2 \pi K_{\mathrm{SGS}}\left(\frac{\ell_{\mathrm{c}}}{\Delta}\right)^{2 \eta}}{6 G \ell_{\mathrm{c}} \rho_{\mathrm{cr}, \mathrm{HC}}} \\
& +\frac{\sqrt{36 \pi G \gamma(\gamma-1) e_{\mathrm{c}} \ell_{\mathrm{c}}^{2} \rho_{\mathrm{cr}, \mathrm{HC}}+4 \pi^{2} K_{\mathrm{SGS}}^{2}\left(\frac{\ell_{\mathrm{c}}}{\Delta}\right)^{4 \eta}}}{6 G \ell_{\mathrm{c}} \rho_{\mathrm{cr}, \mathrm{HC}}}=y_{\mathrm{cut}} \ell_{\mathrm{c}} .
\end{aligned}
$$

Umstellen und Einsetzen der Definitionen von $\mathcal{M}_{\mathrm{c}}$ und $\alpha_{\text {vir }}$ ergibt das kritische Überdichteverhältnis der HC08-13-Modellfamilie

$$
\frac{\rho_{\mathrm{cr}, \mathrm{HC}}}{\rho_{\mathrm{c}, \mathrm{pa}}}=\frac{\pi^{2} \alpha_{\mathrm{vir}}}{5 y_{\mathrm{cut}}^{2} \mathcal{M}_{\mathrm{c}}^{2}}+\frac{\pi^{2} \alpha_{\mathrm{vir}}}{15 y_{\mathrm{cut}}}
$$


wobei der zweite Term in der mff-Version FK13 folgend weggelassen wird. HC08-13 waren die ersten, die einen Freifallzeitgewichtungsfaktor in ihrem Modell einführten, dieser ergab sich schlicht aus der Annahme, dass die relative Geschwindigkeit, mit der einzelne Substrukturen einer Gaswolke kollabieren, durch ihre individuellen, relativen Freifallzeitskalen gegeben ist. $\mathrm{Zu}$ beachten ist, dass genau genommen also alle Modelle der HC08-13-Familie tatsächlich mff-Modelle sind. Die in meinen Simulationen verwendeten Modellkoeffizienten sind der Tabelle $6.1 \mathrm{zu}$ entnehmen.

\subsubsection{PN11-Modell}

Im Rahmen dieser Modellfamilie wird die kritische Überdichte mithilfe der Sprungbedingungen an einem magneto-hydrodynamischen Stoß und der kritischen Masse einer Bonnor-Ebert-Sphäre hergeleitet. Wenn man Effekte magnetischer Felder nicht mit einbezieht, erhalten PN11 für den Dichtesprung an einem Stoß folgenden Ausdruck $^{2}$

$$
\rho_{\mathrm{HD}}=\rho_{\mathrm{c}, \mathrm{pa}} \frac{\mathcal{M}_{\mathrm{c}}^{2}}{4}
$$

woraus die Dicke $\lambda_{\mathrm{HD}}$ der verdichteten Schicht hinter einem Stoß wegen $\rho_{\mathrm{HD}} \lambda_{\mathrm{HD}}=\rho_{\mathrm{c}, \mathrm{pa}} \theta \ell_{\mathrm{c}}$ abgeleitet werden kann

$$
\lambda_{\mathrm{HD}}=\theta \ell_{\mathrm{c}} \frac{4}{\mathcal{M}_{\mathrm{c}}^{2}} .
$$

$\theta \ell_{\mathrm{c}} \leq \ell_{\mathrm{c}}$ kann als die Injektionsskala von Turbulenz in der Gaswolke angesehen werden. Eine Gasverdichtung wird als instabil erachtet, wenn die in einer Kugel mit Durchmesser $\lambda_{\mathrm{HD}}$ eingeschlossene Masse die kritische Masse $M_{\mathrm{BE}}$ einer marginal stabilen Bonnor-Ebert-Sphäre hat oder überschreitet

$$
M_{\mathrm{BE}}=1,182 \rho_{\mathrm{cr}, \mathrm{PN}}\left[\frac{\gamma(\gamma-1) e_{\mathrm{c}}}{G \rho_{\mathrm{cr}, \mathrm{PN}}}\right]^{3 / 2}=M_{\mathrm{cr}, \mathrm{PN}} \leq \frac{4 \pi}{3} \rho_{\mathrm{cr}, \mathrm{PN}}\left(\frac{\lambda_{\mathrm{HD}}}{2}\right)^{3} .
$$

Durch Umstellen und Einsetzen der Definitionen von $\lambda_{\mathrm{HD}}, \mathcal{M}_{\mathrm{c}}$ und $\alpha_{\text {vir }}$ ergibt sich das kritische Überdichteverhältnis der PN11-Modellfamilie als

$$
\rho_{\text {crit }, \mathrm{PN}} / \rho_{\mathrm{c}, \mathrm{pa}}=0,067 \times \theta^{-2} \alpha_{\mathrm{vir}} \mathcal{M}_{\mathrm{c}} .
$$

PN11 verwenden in ihrem Modell den bezüglich der pdf $(x)$-Integration konstanten Freifallzeitgewichtungsfaktor $f_{\mathrm{ff}, \mathrm{PN}}=\left(\rho_{\mathrm{c}, \mathrm{pa}} / \rho_{\mathrm{cr}, \mathrm{PN}}\right)^{1 / 2}$, sodass alle überkritischen Dichten um diesen Faktor erhöht eingehen. Sie verwenden abgesehen von $\epsilon_{\text {core }}$ jedoch keinen Vorfaktor $f_{\text {cal,PN }}$, also setze ich in meinen Simulationen $f_{\text {cal,PN }}=1,0$ beziehungsweise die von FK13 ermittelten Werte. Da die turbulente Geschwindigkeitsdispersion im Rahmen von MIST auf $\ell_{c}$ skaliert wird, was als Injektionsskala der Turbulenz in der kalten Phase aufgefasst wird, nutze ich im Falle der FK13kalibrierten Modelle $\theta=1,0$ statt der von FK13 bestimmten Werte.

\footnotetext{
${ }^{2}$ Das Subskript 'HD' deutet an, das nur reine Hydrodynamik betrachtet wird, im Gegensatz zu Magnetohydrodynamik ('MHD').
} 


\subsubsection{PHN12-Modell}

Im Gegensatz zu den zuvor vorgestellten Modellfamilien KM05, HC08-13 und PN11 fußst das Modell von PHN12 nicht auf theoretischen Modellannahmen und einer analytischen Herleitung, sondern stellt eine relativ simpel gehaltene, analytische Anpassung an Ergebnisse von Simulationen getriebener isothermer Turbulenz mit Eigengravitation in periodischen Boxen dar. So schlagen PHN12 das empirische Sternentstehungsgesetz

$$
\epsilon_{\mathrm{PHN}}=\epsilon_{\mathrm{core}} \exp \left(-1,6 \times \frac{\tau_{\mathrm{ff}}}{\tau_{\mathrm{dyn}}}\right)
$$

vor, wobei die dynamische Zeitskala $\tau_{\text {dyn }}$ als

$$
\tau_{\mathrm{dyn}}=\frac{\ell_{\mathrm{c}}^{1-\eta} \Delta^{\eta}}{2^{3 / 2} K_{\mathrm{SGS}}^{1 / 2}}
$$

definiert ist. So ergibt sich nach Einsetzen der Definition von $\alpha_{\text {vir }}$

$$
\epsilon_{\mathrm{PHN}}=\epsilon_{\mathrm{core}} \exp \left(-0,003 \times \pi^{2} \alpha_{\mathrm{vir}}\right)
$$




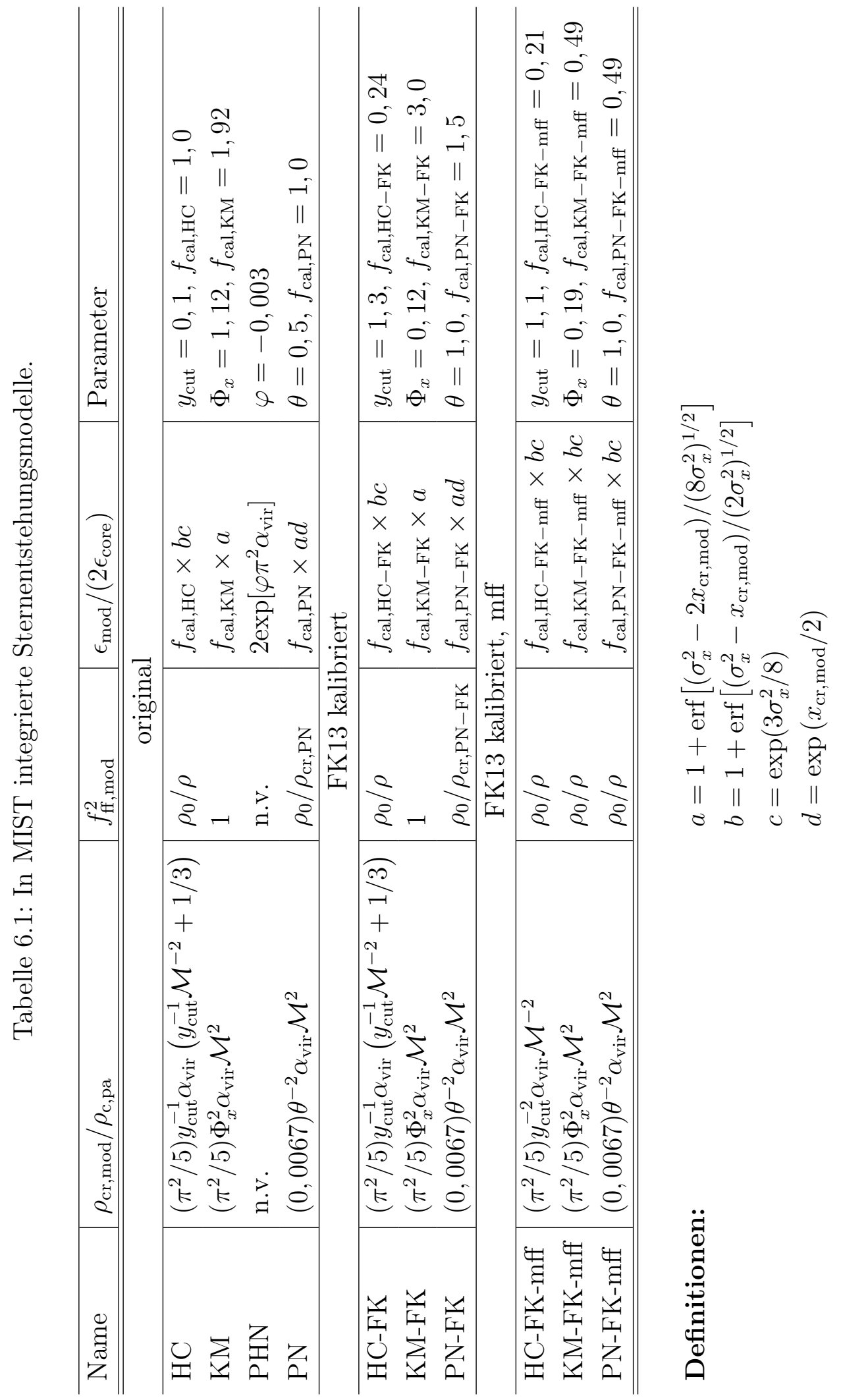




\subsection{Simulationen}

Insgesamt habe ich zehn Programmläufe mit Nyx (Almgren u. a., 2013) unter Verwendung des SGS-Modells für nicht aufgelöste Turbulenz von SF11 und SA14 und des in BS14 vorgestellten Modells MIST durchgeführt, wobei in jedem Durchlauf ein anderes der in Tabelle 6.1 aufgeführten Sternentstehungsmodelle zur Anwendung kam. Als Anfangsbedingung verwendete ich die Simulationsdaten des in BS14 vorgestellten, mit 'ref' bezeichneten Programmlaufs bei einem simulierten Alter der isolierten Scheibengalaxie von 1 Ga. Dies stellt sicher, dass sich die Galaxie zum Beginn der Simulationen der $\epsilon_{\text {mod }^{-}}$Gruppe in einem bereits durch das Zusammenwirken von Sternentstehung und stellarer Rückwirkung selbstregulierten Zustand befindet. Alle Simulationen wurden bis zu einem Alter von 1,4 Ga fortentwickelt, dies wiederum soll sicherstellen, dass die Galaxie wieder einen unter den Bedingungen des neuen Sternentstehungsmodells selbstregulierten Zustand erreicht hat, sofern ein solcher Zustand mit absehbarem Aufwand erreichbar ist. Die 0,4 Ga entsprechen etwa der für einen Orbit im Abstand von $10 \mathrm{kpc}$ vom Zentrum der galaktischen Scheibe benötigten Zeit - oder dem zehnfachen der Zeitspanne, nach der eine neu entstandene Sternenpopulation nicht mehr aktiv auf das Gas über SN-Auswürfe einwirkt.

\subsection{Ergebnisse}

\subsubsection{Sternentstehungsraten}

Wie in Abb. $6.1 \mathrm{zu}$ sehen pegelt sich die globale Sternentstehungsrate $\dot{M}_{\mathrm{SF}}$ nach einer kurzen Übergangszeit von weniger als $50 \mathrm{Ma}$, in der sich die Struktur der Gasscheibe an die neuen Gegebenheiten anpasst, für die meisten getesteten Modelle (genauer gesagt 'PN', 'PN-FK', 'PN-FK-mff', 'HC', 'HC-FK', 'HC-FK-mff' sowie 'KM-FK-mff') auf mittlere Werte zwischen 2 und $3 \mathrm{M}_{\odot} a^{-1}$ ein, wobei sie jedoch stark um bis zu $\sim 2 \mathrm{M}_{\odot} a^{-1}$ schwankt. Die Sternentstehungsrate in diesen Simulationen entwickelt sich dabei ähnlich der Sternentstehungsrate des bereits im von BS14 vorgestellten 'ref' genannten Programmlauf verwendeten Sternentstehungsmodells (schwarz durchgezogene Linie), das hier mit 'PN-FK' bezeichnet wird. Die Entwicklung der mittleren Sternentstehungsrate lässt sich im unteren Teil von Abb. 6.1 besser nachvollziehen, hier wurden die globalen Sternentstehungsraten nämlich gleitend über 80 Ma gemittelt, sodass die Variationen mit kürzeren Zeitskalen weggeglättet wurden. In den Programmläufen mit 'KM'-, 'KM-FK'- und 'PHN'-Modell steigt die mittlere globale Sternentstehungsrate über den gesamten von den Simulationsläufen abgedeckten Zeitraum stetig an, nachdem sie zu Beginn (bei $1 \mathrm{Ga}$ ) weit unter der Gleichgewichtsrate des 'PN-FK'-Modells starten. Es scheint so, als ob sich auch $\dot{M}_{\mathrm{SF}}$ im Lauf mit dem 'PHN'-Modell langsam auf Niveau des 'PN-FK'-Modells einpegelt. 

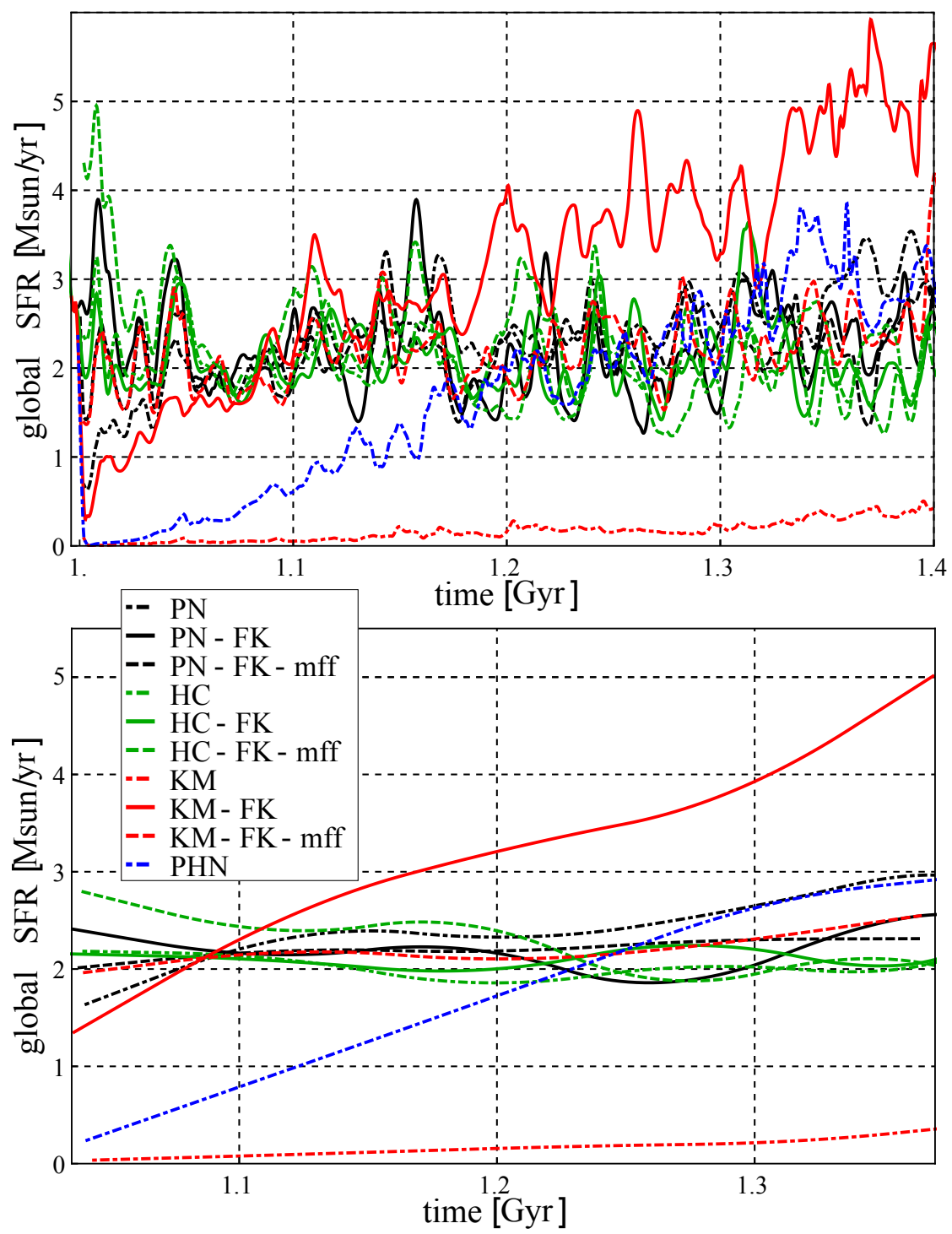

Abbildung 6.1: Oben: Globale Sternentstehungsrate $\dot{M}_{\mathrm{SF}}$ über Simulationszeit für die verschiedenen Simulationsläufe mit jeweils unterschiedlichem Sternentstehungsmodell für die kalte Phase. Der Verlauf $\dot{M}_{\mathrm{SF}}$ von Programmläufen mit PN11-artigem Sternentstehungsmodell ist schwarz, mit HC08-13-artigen grün, mit KM05-artigen rot und PHN12-artige blau, dargestellt. Dabei steht eine punkt-gestrichelte Linie für das jeweilige Modell in seiner originalen Form, eine durchgezogene Linie für das jeweilige Modell in der Formulierung vom FK13 mit einfachem Freifallzeitkoeffizienten (sff), und eine gestrichelte Linie für das jeweilige Modell in der Formulierung vom FK13 mit angepasstem Freifallzeitkoeffizienten (mff). Unten: Gleitend über einen Zeitraum von 80 Ma gemittelte Sternentstehungsrate $\dot{M}_{\mathrm{SF}}$ über Simulationszeit für die verschiedenen Simulationsläufe mit jeweils unterschiedlichem Sternentstehungsmodell für die kalte Phase. Linienarten und -farben wie oben 
Doch es besteht die Möglichkeit, dass eine langfristigere Variation der $\dot{M}_{\mathrm{SF}}$ nur den Anschein eines Absättigens zu einem stabilen selbstregulierten Zustand hin erweckt. Für die anderen beiden Modelle lässt sich kein Hinweis auf die Stabilisierung von $\dot{M}_{\mathrm{SF}}$ erkennen, wobei im Falle von 'KM' die Sternentstehungsrate im Verlaufe der Simulation nicht über $0,5 \mathrm{M}_{\odot} a^{-1}$ hinauswächst, aber im Falle von 'KM-FK' sogar bis auf das zehnfache dessen $\left(\sim 5 \mathrm{M}_{\odot} a^{-1}\right)$ anwächst. Es ist also festzustellen, dass sich die Modellgalaxie unter Verwendung der Modelle 'KM', 'KM-FK' und 'PHN' - auch nach einer Zeitspanne von 0,4 Ga - nicht in einem statistisch stabilen, selbstregulierten Zustand entwickelt hat, oder dies zumindest fraglich bleibt. Bei Verwendung der übrigen Modelle in dieser Studie befindet sich die Modellgalaxie innerhalb kurzer Zeit in einem stabilen selbstregulierten Zustand. Die nachfolgenden Entwicklungen der Sternentstehungsraten sind untereinander sehr ähnlich.

Die Zeitskalen der meisten im oberen Teil von Abb. 6.1 sichtbaren Variationen decken sich mit denen, die schon in BS14 beschrieben wurden. Sie sind auf den Lebenszyklus individueller, sternbildender Regionen zurückzuführen, der $\sim 10$ bis 30 Ma währt. Diese Art von Variation wird nicht maßgeblich durch das verwendete Sternentstehungsmodell vorgegeben, sondern von den Zeitskalen, die sich aus der zeitlichen Entwicklung der massereichen Sterne, namentlich deren Vergehen in SN-Explosionen, ergeben.

Der Verlauf der Sternentstehung für die nicht absättigenden Modelle zeigt Variationen (besonders zu späteren Zeiten hin), die mit Zeitskalen von nur wenigen Ma wesentlich kürzer sind als die oben erwähnten, mit dem Lebenszyklus sternbildender Gebiete verbundenen Zeitskalen. Diese Art von Variation kann als Indiz für die bereits in BS14 festgestellte intermittierende Sternentstehung, die in massiven Ansammlungen von Sternen stattfindet, angesehen werden. Lokal dominieren diese Ansammlungen das galaktische Gravitationspotential und sind so immer wieder in der Lage Gas zu akkretieren, welches dort aufgrund des herrschenden hohen Gasdruckes äußerst schnell in Sterne verwandelt werden kann. Dies geschieht so effizient, dass das eingefallene Gas schneller verbraucht ist als weiteres Gas nachströmen kann. Dadurch wird die Sternentstehung fast ebenso abrupt gedrosselt wird wie sie kurz zuvor angelaufen ist.

\subsubsection{Struktur der Gasscheiben}

Vergleicht man die Struktur der Gasscheiben in den verschiedenen Simulationen, wie zum Beispiel in der in Abb. 6.2 zu sehenden Zusammenschau der senkrecht zur Scheibenebene projizierten Gasdichte $\rho$ in einer $20 \times 20 \mathrm{kpc}$ umfassenden Region um das Zentrum am Ende der Simulationen, so stechen auch hier die bereits bei der Betrachtung der globalen Sternentstehungsrate als abweichend aufgefallenen Modelle 


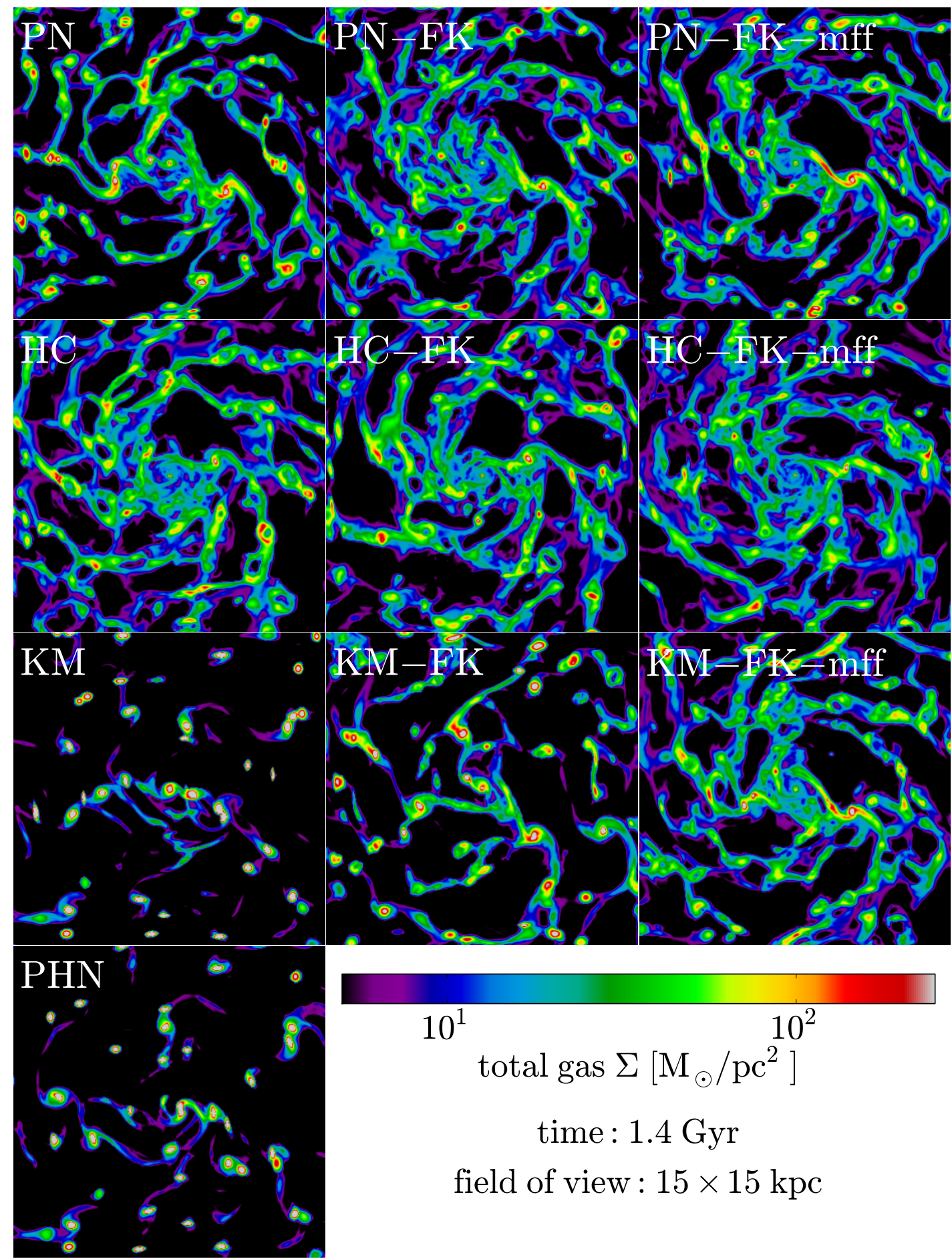

Abbildung 6.2: Zusammenschau von logarithmisch farbkodiert dargestellten Projektionen der Gesamtgasdichte $\rho$ entlang der Drehimpulsachse der Galaxienscheibe in einem zentralen $15 \times 15 k p c$ großen Bereich der Scheibe. Die einzelnen Projektionen stellen den Zustand in den benannten Simulationsläufen bei einer Simulationszeit von $\sim 1,4$ Ga dar. 

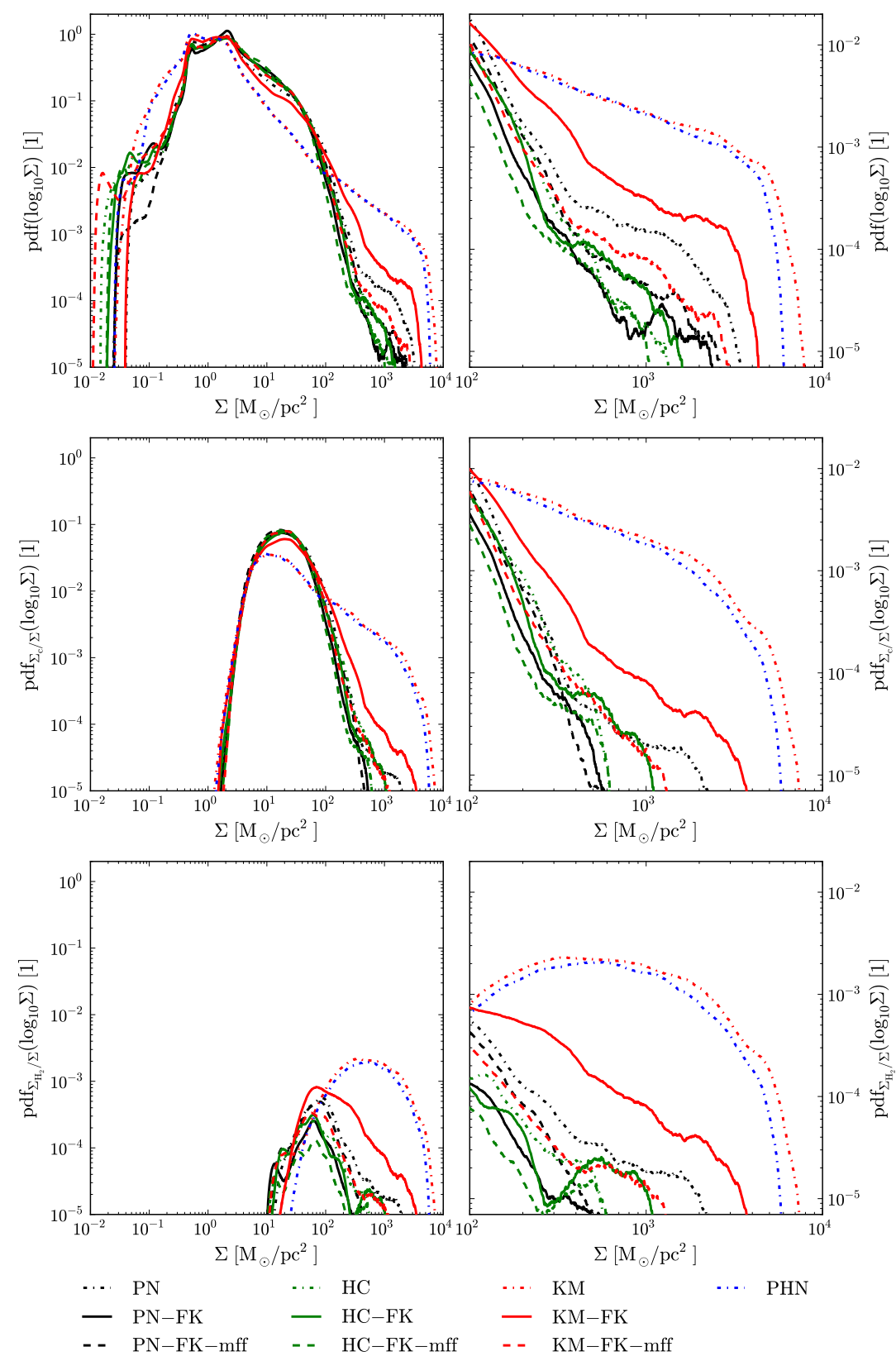

$\begin{array}{ll}- & \mathrm{PN}-\mathrm{FK} \\ - & \mathrm{PN}-\mathrm{FK}-\mathrm{m} f\end{array}$

-... $\mathrm{KM}$

-.. PHN

- KM-FK

Abbildung 6.3: Oben links: Flächengewichtete Wahrscheinlichkeitsdichteverteilung $\operatorname{pdf}\left(\log _{10} \Sigma\right)$ des Logarithmus der Gassäulendichte in der Scheibe über der Gassäulendichte $\Sigma$ für die Simulationsläufe mit verschiedenen Sternentstehungsmodellen bei einem Alter von $\sim 1,4$ Ga. Linienarten und -farben wie in Abb. 6.1. Oben rechts: Ausschnittvergrößerung des Diagramms von oben links für $\Sigma>10^{2} \mathrm{M}_{\odot} p c^{-3}$. Mitte links: Mit dem Massenanteil $\Sigma_{\mathrm{c}} / \Sigma$ der kalten Phase gewichtete Wahrscheinlichkeitsdichteverteilung $\operatorname{pdf}_{\Sigma_{\mathrm{c}} / \Sigma}\left(\log _{10} \Sigma\right)$ über $\Sigma$. Mitte rechts: Ausschnittvergrößerung des Diagramms von mittig links für $\Sigma>10^{2} \mathrm{M}_{\odot} p c^{-3}$. Unten links: Mit dem Massenanteil $\Sigma_{\mathrm{H}_{2}} / \Sigma$ des molekularen Gases gewichtete Wahrscheinlichkeitsdichteverteilung $\operatorname{pdf}_{\Sigma_{\mathrm{c}} / \Sigma}\left(\log _{10} \Sigma\right)$ über $\Sigma$. Unten rechts: Ausschnittvergrößerung des Diagramms von oben links für $\Sigma>10^{2} \mathrm{M}_{\odot} p c^{-3}$. 
'KM', 'KM-FK' und 'PHN' heraus, in geringerem Maße aber auch 'PN'. Die entsprechenden Gasscheiben der ersten drei zeigen große Bereiche mit geringer Gasdichte. Das Gas ist in diesen Fällen nämlich in massiven Klumpen konzentriert, während Gas mittlerer Dichten ausschließlich in Gezeitenarmen zu finden ist, die bei engen Begegnungen oder Verschmelzungen solcher Klumpen entstehen. In den Gasscheiben der übrigen Simulationsläufe ist einerseits kaum derart verdichtetes Gas anzutreffen, und andererseits wird viel Raum von Gas mittlerer Dichten eingenommen. Dieses Gas mittlerer Dichte befindet sich hauptsächlich in sprialartig aufgelockerten Strukturen, die sich netzartig, durch expandierende Sternentstehungsregionen gestört, über die Scheibe ausbreiten. Qualitativ ergibt sich für diese Fälle dasselbe Bild wie bereits im 'ref' Programmlauf nach 1 Ga (vergleiche hierzu mit Abb. 5.3). Die Gasscheiben im 'PN'- und 'KM-FK'-Programmlauf sind nicht eindeutig der Gruppe der klumpigen oder der aufgelockerten Scheiben zuzuordnen, sondern liegen dazwischen, wobei 'KM-FK' eher klumpiger ausgeprägt ist als 'PN'.

Genauer differenzieren lässt sich dies anhand der in Abbildung 6.3 gezeigten flächengewichteten, beziehungsweise mit dem Kaltphasenanteil $\Sigma_{\mathrm{c}} / \Sigma$ gewichteten Säulendichteverteilungen pdf $\left[\log _{10}(\Sigma)\right]$ und $\operatorname{pdf}_{\Sigma_{\mathrm{c}} / \Sigma}\left[\log _{10}(\Sigma)\right]$, die durch

$$
\int_{-\infty}^{\infty} \operatorname{pdf}\left[\log _{10}(\Sigma)\right] \mathrm{d}\left[\log _{10}(\Sigma)\right]=\frac{M}{M}=1
$$

beziehungsweise

$$
\int_{-\infty}^{\infty} \operatorname{pdf}_{\frac{\Sigma_{c}}{\Sigma}}\left[\log _{10}(\Sigma)\right] \mathrm{d}\left[\log _{10}(\Sigma)\right]=\frac{M_{\mathrm{c}}}{M}
$$

normiert sind. Im oberen linken Teil von Abb. 6.3 lassen sich vier verschiedene Dichteregimes innerhalb des Gases erkennen, nachfolgend von kleinen zu großen $\Sigma$ hin aufgelistet.

- a) $\Sigma \lesssim 2 \times 10^{-1} \mathrm{M}_{\odot} \mathrm{pc}^{-2}$ : In diesem Bereich findet sich extrem heißes, verdünntes Gas wie es von SN-Explosionen freigesetzt wird.

- b) $2 \times 10^{-1} \mathrm{M}_{\odot} \mathrm{pc}^{-2} \lesssim \Sigma \lesssim 3 \times 10^{0} \mathrm{M}_{\odot} \mathrm{pc}^{-2}$ : Im Gas dieses Regimes hat sich im Mittel ein Gleichgewicht von Heizen durch photoelektrischen Effekt an Staub sowie durch Dissipation turbulenter Energie und radiativem Kühlen eingestellt. Gut zu erkennen sind die zwei Maxima, die in allen Verteilungsfunktionen an gleicher Stelle auftauchen. Diese markieren bei welchen Dichten sich der Übergang zwischen ionisiertem und neutralem Wasserstoff beziehungsweise Helium vollzieht. Für Helium liegt der Übergang bei $\approx 5 \times 10^{-1} \mathrm{M}_{\odot} \mathrm{pc}^{-2}$ und für Wasserstoff bei $\approx 2 \times 10^{0} \mathrm{M}_{\odot} \mathrm{pc}^{-2}$, was auch der Grund ist, weshalb kaum Kaltphasengas unterhalb dieser Dichte vorkommen kann $\left(\rho_{\mathrm{w}} \approx \rho\right)$, da Neutralität des Gases eine Voraussetzung für die Entstehung kalter Phase durch thermische Instabilität ist (siehe Auftragung im mittleren linken Teil von Abb. 6.3). 
- c) $3 \times 10^{0} \mathrm{M}_{\odot} \mathrm{pc}^{-2} \lesssim \Sigma \lesssim 10^{2} \mathrm{M}_{\odot} \mathrm{pc}^{-2}$ : Hier schließst sich nun der Bereich kühlen, neutralen Gases mittlerer Dichten an, in dem auch die kalte Phase $\rho_{\mathrm{c}}$ neben der warmen $\rho_{\mathrm{w}}$ des MIST-Modells existieren kann. Die Verteilung der kalten Phase nimmt eine log-normale Form an, deren Maximum bei $\Sigma \approx$ $2 \times 10^{1} \mathrm{M}_{\odot} \mathrm{pc}^{-2}$ liegt. Auch in pdf $\left[\log _{10}(\Sigma)\right]$ lässt sich die Auswirkung der Exisistenz einer kalten Phase als Verbreiterung der bereits abfallenden Schulter der Verteilung zu hohen $\Sigma$ hin ausmachen. Oberhalb von $\approx 1 \times 10^{1} \mathrm{M}_{\odot} \mathrm{pc}^{-2}$ existiert, wie im unteren linken Teil von Abb. 6.3 ersichtlich, innerhalb der kalten Phase auch molekulares Gas.

- d) $\Sigma \gtrsim 10^{2} \mathrm{M}_{\odot} \mathrm{pc}^{-2}: \mathrm{Zu}$ hohen Dichten hin erscheint ein an die Schulter der Dichteverteilungen angesetzter Schwanz, der ein Resultat der in diesem Dichtebereich nicht mehr vernachlässigbaren gemeinsamen Eigengravitation des Gases und der Sterne ist. Es sind die in Abb. 6.2 prominent sichtbaren, mehr oder weniger stark gravitativ gebundenen Gasansammlungen, die für eine Bevölkerung dieses Bereiches der Säulendichteverteilungen sorgen. Der Anteil des molekularen Gases, aus dem Sterne gebildet werden können, ist hier dominant innerhalb der kalten Phase. Jedoch fällt auf, dass der Anteil kalten Gases klar hinter der Gesamtdichte zurückbleibt, was durch stellare Rückwirkung verursachte Evaporation der kalten Phase bewirkt wird.

Die größten Unterschiede zwischen den Sternentstehungsmodellen zeigen sich im Regime d), in welchem auch die meiste Sternentstehung stattfindet. So ist der Hochdichtenschwanz der Verteilungen für die Modelle 'PHN' und 'KM' besonders ausgeprägt, und die Maxima der entsprechenden molekularen Verteilungen liegen mit $\approx 6 \times 10^{1} \mathrm{M}_{\odot} \mathrm{pc}^{-2}$ etwa eine Größenordnung über denen der übrigen Verteilungen. Auch der Mangel an Gas mittlerer Dichte sowie das gehäufte Auftreten von Gas minderer Dichte in diesen Programmläufen ist in den Regimes b) beziehungsweise c) leicht erkennbar. Die Verteilungen aus 'KM-FK' und 'PN' weisen gegenüber den restlichen Modellen einen signifikant stärker betonten Schwanz auf, wenn auch weit weniger ausgeprägt als im Falle von 'PHN' oder 'KM'. Die Unterschiede zwischen den Modellen 'PN-FK' , 'PN-FK-mff', 'HC', HC-FK', HC-FK-mff' und 'KM-FKmff' untereinander sind dagegen als marginal anzusehen.

\subsubsection{Sternentstehungsrelationen}

Für die meisten der untersuchten Modelle stellt sich, wie in Abb. $6.4 \mathrm{zu}$ sehen, die bereits für die Simulationen in BS14 festgestellte robuste, lineare Relation zwischen Sternentstehungssäulendichte $\dot{\Sigma}_{\mathrm{SF}}$ und molekularer Säulendichte $\Sigma_{\mathrm{H}_{2}}$ ein, obwohl die inferierten Sternentstehungszeitskalen des molekularen Gases zwischen den einzelnen Modellen von $\approx 80 \mathrm{Ma}$ bis $\mathrm{zu} \approx 0,5 \mathrm{Ga}$ variieren. Gerade die in den vorigen 


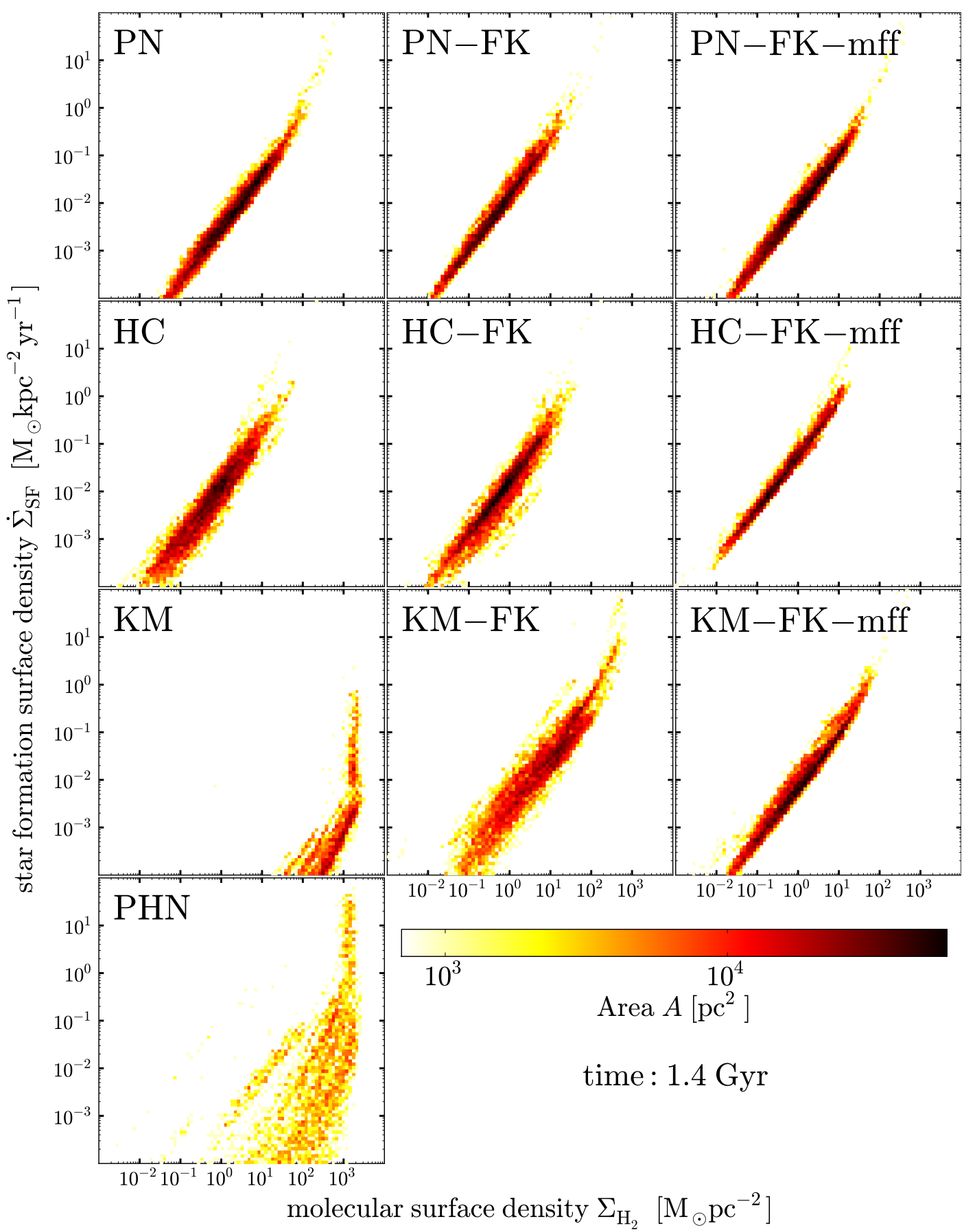

Abbildung 6.4: Zusammenschau von logarithmisch farbkodiert dargestellten zweidimensionalen flächengewichteten Histogrammen bezüglich der Sternentstehungssäulendichte $\dot{\Sigma}_{\mathrm{s}}$ und der Säulendichte dichten, molekularen Gases $\Sigma_{\mathrm{H}_{2}}$ für die benannten Simulationsläufe bei einer Simulationszeit von $\sim 1,4$ Ga. 


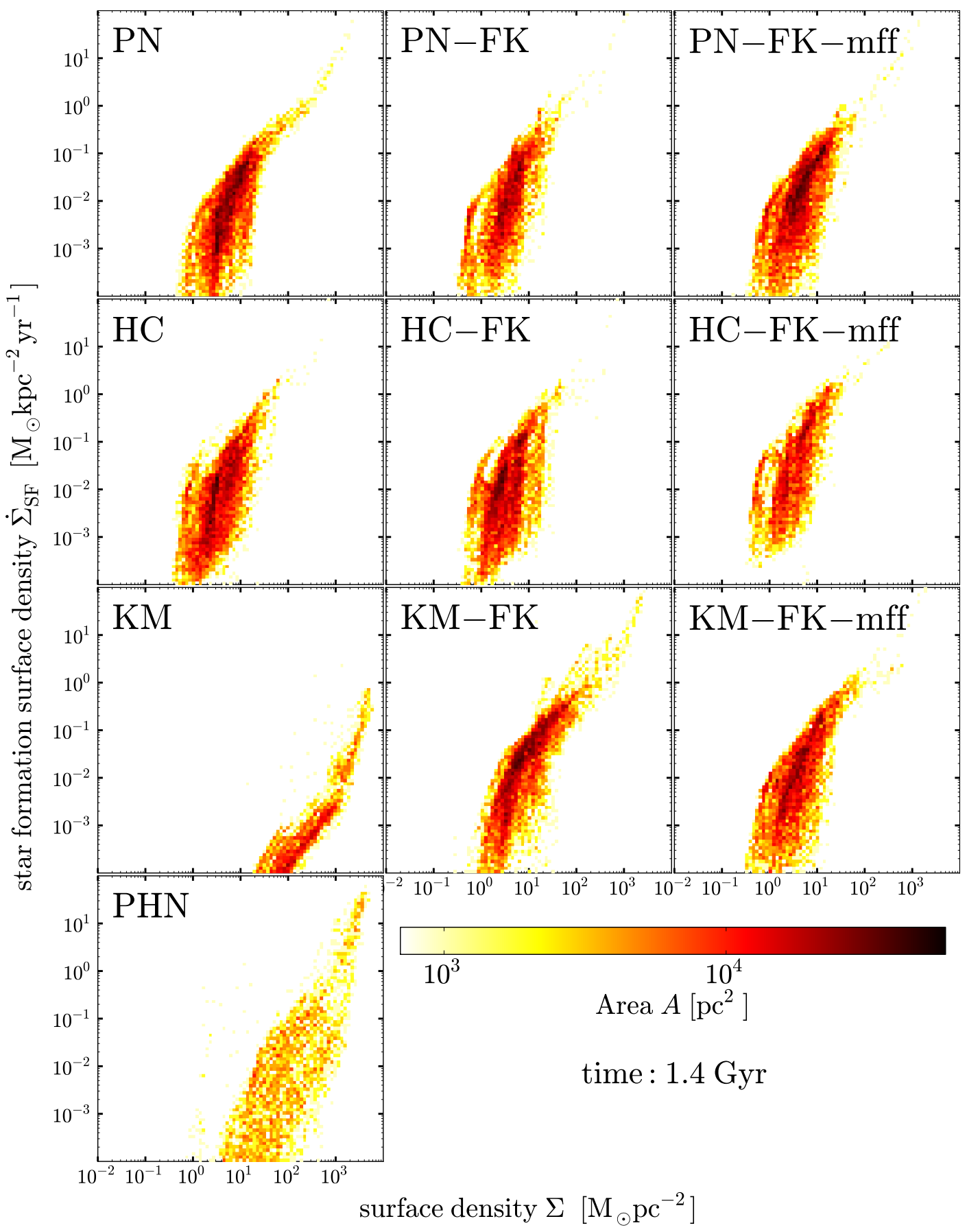

Abbildung 6.5: Zusammenschau von logarithmisch farbkodiert dargestellten zweidimensionalen flächengewichteten Histogrammen bezüglich der Sternentstehungssäulendichte $\dot{\Sigma}_{\mathrm{s}}$ und der Gassäulendichte $\Sigma$ für die benannten Simulationsläufe bei einer Simulationszeit von $\sim 1,4$ Ga. 
Abschnitten bereits als abweichend identifizierten Modelle 'PHN' und 'KM' stellen auch hier wieder Ausnahmen dar. Einerseits liegen die aus dem linear korrelierteren Teil ihres $\dot{\Sigma}_{\mathrm{SF}^{-}} \Sigma_{\mathrm{H}_{2}}$-Diagramms inferierten Sternentstehungszeitskalen mit $\sim 1000 \mathrm{Ga}$ weit außerhalb dessen, was mit Beobachtungen vereinbar wäre, andererseits knickt die Verteilung oberhalb von $\Sigma_{\mathrm{H}_{2}} \approx 10^{3} \mathrm{M}_{\odot} \mathrm{pc}^{-2}$ steil nach oben ab. Der Grund hierfür ist nicht klar identifizierbar. Dies geht jedoch mit lokal dauerhafter Verletzung des Toomre-Kriteriums (Toomre, 1964) für die numerische Mindestauflösung der (effektiven) Jeanslänge einher, und der geringen Größe $\ell_{c}$ der Kaltphasenklumpen, die ebenfalls über eine Jeanslänge definiert ist, was aber nicht notwendigerweise ursächlich ist. Leichte Anzeichen eines solchen Abknickens zu einer wesentlich steileren $\dot{\Sigma}_{\mathrm{SF}^{-}} \Sigma_{\mathrm{H}_{2}}$-Relation sind auch in den entsprechenden Diagrammen zu 'KM-FK' und 'PN' erkennbar.

Auch die in Abb. 6.5 gezeigten zweidimensionalen Histogramme der $\dot{\Sigma}_{\mathrm{SF}^{-}} \Sigma$-Relationen zeigen das von BS14 bereits beschriebene Bild. Es zeichnet sich durch ein Abschneiden der Sternentstehung unterhalb von Gassäulendichten $\Sigma \approx 10^{0} \mathrm{M}_{\odot} \mathrm{pc}^{-2}$ wegen Mangels an molekularem Gas und einen steilen Anstieg darüber aus, der gegen höhere Dichten allmählich abflacht. Die Bereiche hoher Säulendichten sind zwar wie zum Beispiel bei 'PN-FK' nur schwach bevölkert, dennoch kann aus dem Schwanz der $\dot{\Sigma}_{\mathrm{SF}^{-}} \Sigma$-Verteilung eine KS-Relation mit $\alpha_{\mathrm{KS}} \approx 2$ abgelesen werden. Dieser Wert wird in Beobachtungen bei Sternentstehungsausbrüchen (star bursts) festgestellt. Vermutlich liegt dies daran, dass die simulierten Galaxien auch nach 1,4 Ga Entwicklung noch stark von Gas und seiner Dynamik dominiert sind, was auch für 'star bursts' gilt.

Die schon benannten Modelle 'PN', 'KM', 'KM-FK' und 'PHN' zeigen auch hier ein Abknicken zu einer wesentlich steileren $\dot{\Sigma}_{\mathrm{SF}^{-}} \Sigma$-Relation für hohe Säulendichten, was zu erwarten ist, da der molekulare Anteil des Gases in diesem Bereich dominant ist.

\subsubsection{Sternentstehung und Turbulenz}

Sternentstehung findet für alle in dieser Studie einbezogenen Modelle, wie es Abb. 5.6 $\mathrm{zu}$ entnehmen ist, schwerpunktmäßig bei spezifischen SGS-Energien um $K_{\mathrm{SGS}} \approx$ $100 \mathrm{~km}^{2} \mathrm{~s}^{-1}$ statt, was in etwa einer Geschwindigkeitsdispersion von $\sigma_{\mathbf{u}} \approx 10 \mathrm{~km} \mathrm{~s}^{-1}$ entspricht. Dies deckt sich auch mit dem mit Beobachtungen (zum Beispiel Leroy u.a., 2008; Shetty u.a., 2012; Stilp u.a., 2013) übereinstimmenden Ergebnis von BS14. Die Stärke der nicht aufgelösten Turbulenz in aktiv sternbildenden Regionen der Galaxienscheibe wird hauptsächlich über die stellare Rückwirkung, den dadurch bedingten Massenaustausch zwischen den Phasen innerhalb MIST und das angenommene Gleichgewicht des effektiven Druckes zwischen den Phasen reguliert. Das verwendete Sternentstehungsmodell spielt eine untergeordnete Rolle in Bezug auf 


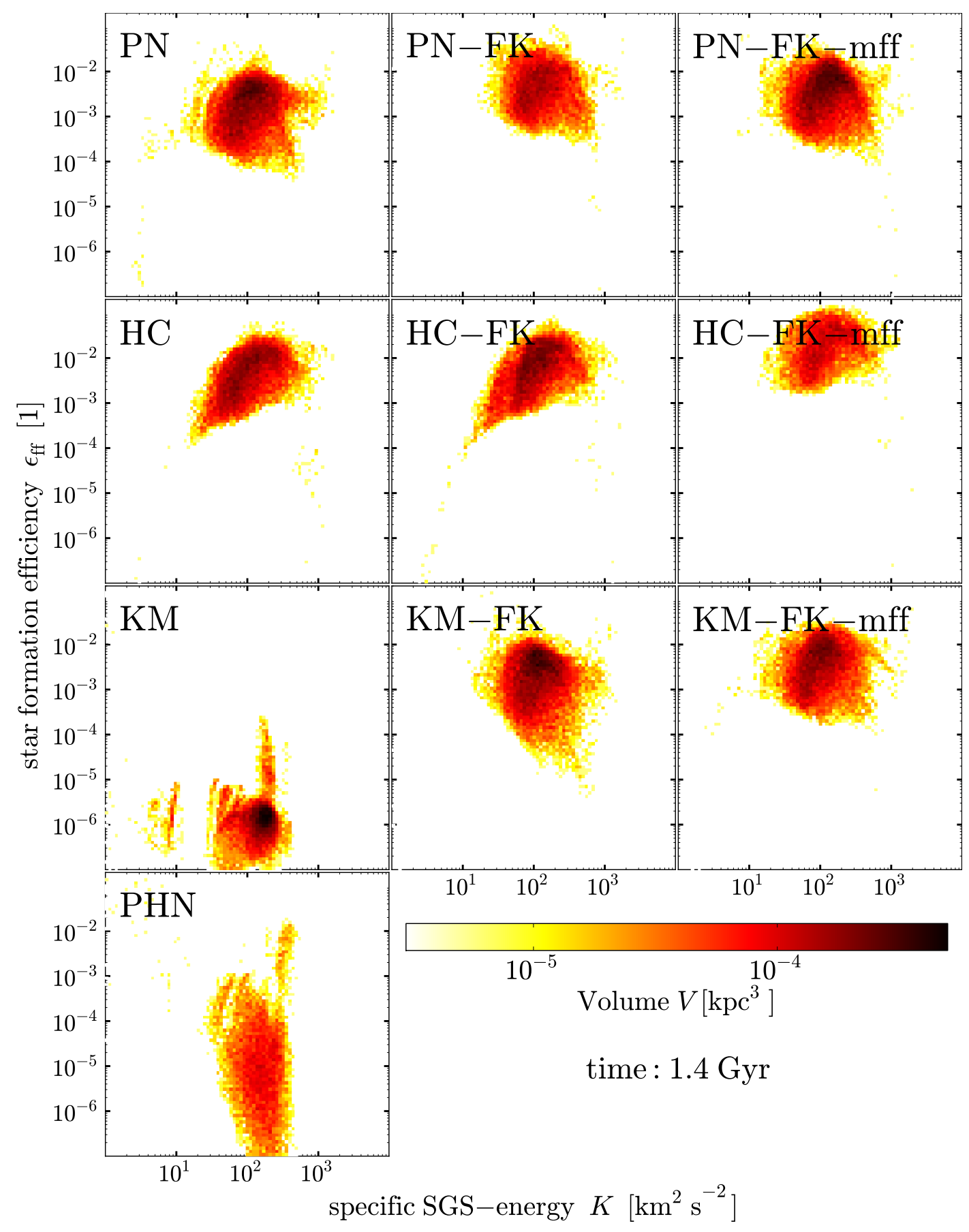

Abbildung 6.6: Zusammenschau von logarithmisch farbkodiert dargestellten zweidimensionalen flächengewichteten Histogrammen bezüglich der Sternentstehungseffizienz $\epsilon_{\mathrm{ff}}$ und der spezifischen turbulenten SGS-Energie $K_{\mathrm{SGS}}$ für die benannten Simulationsläufe bei einer Simulationszeit von $\sim 1,4 \mathrm{Ga}$, wie im linken Teil der Abb. 5.6. 


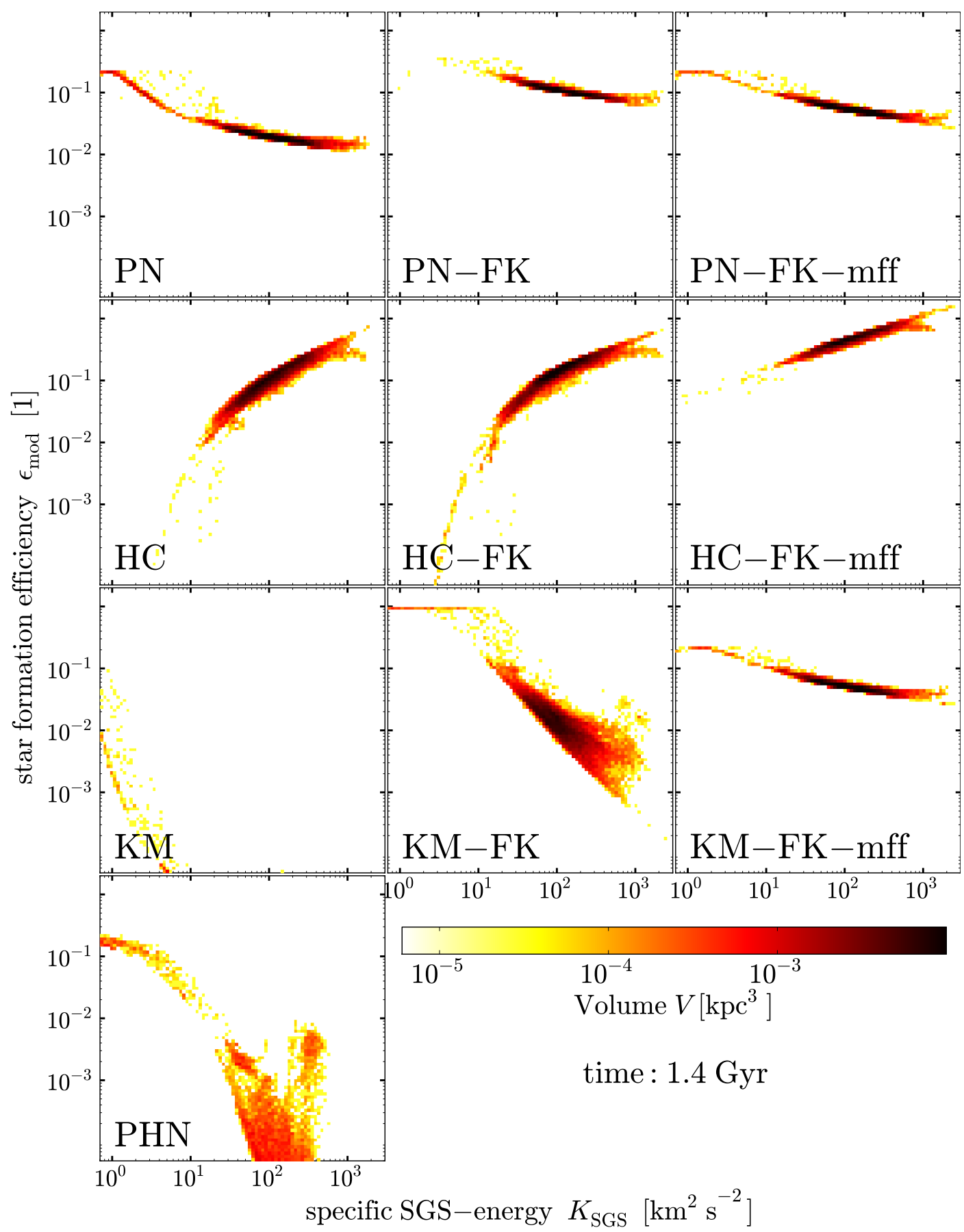

Abbildung 6.7: Zusammenschau von logarithmisch farbkodiert dargestellten zweidimensionalen flächengewichteten Histogrammen bezüglich der Sternentstehungseffizienz $\epsilon_{\text {mod }}$ in der kalten Phase und der spezifischen turbulenten SGS-Energie $K_{\text {SGS }}$ für die benannten Simulationsläufe bei einer Simulationszeit von $\sim$ 1, 4 Ga. 
die Turbulenzstärke. Die Sternentstehungseffizienz bezüglich des gesamten Gasinhalts eines Volumenelements ist gegeben durch

$$
\epsilon_{\mathrm{ff}}=\epsilon_{\mathrm{mod}} \frac{f_{\mathrm{H}_{2}} \rho_{\mathrm{c}} \tau_{\mathrm{ff}}(\rho)}{\rho \tau_{\mathrm{ff}}\left(\rho_{\mathrm{c}, \mathrm{pa}}\right)}
$$

wie bereits in Kapitel 4.1.2 oder BS12 definiert. Das Hauptgewicht der Sternentstehungseffizienz $\epsilon_{\mathrm{ff}}$ liegt für die meisten Modelle im Bereich $\epsilon_{\mathrm{ff}}=10^{-3} \ldots 10^{-2}$ (siehe Abb. 5.6). Nur 'PHN' und 'KM' weisen weit geringere Effizienzen $\epsilon_{\mathrm{ff}}$ auf. Im Falle von 'PHN' ist zu bemerken, dass die globale Sternentstehungsrate bei 1,4 Ga Simulationsalter - trotz weit geringerem $\epsilon_{\mathrm{ff}}-$ mit der von 'PN-FK' beispielsweise vergleichbar ist. Sternentstehung findet in 'PHN' nur bei in Regionen äußerst hoher Dichten statt, weshalb die Effizienz pro Freifallzeit zwar gering scheint, aber die Freifallzeitskala wegen der hohen Dichte dort besonders kurz ist.

Die in Abb. 5.6 gezeigten $\epsilon_{\mathrm{ff}}-K_{\mathrm{SGS}}$-Verteilungen für die Modelle der PN11-Familie sowie 'KM-FK' und 'KM-FK-mff' sind von rundlicher Form und erstrecken sich fast über zwei Dekaden sowohl in der $\epsilon_{\mathrm{ff}}$ - als auch in der $K_{\mathrm{SGS}}$-Richtung der $\epsilon_{\mathrm{ff}}$ $K_{\mathrm{SGS}}$-Ebene. Dabei zeigen die meisten eine Ausbuchtung zu kleinen $\epsilon_{\mathrm{ff}}{ }^{-}$, aber großen $K_{\mathrm{SGS}}$-Werten hin. Die Modelle der HC08-13 Familie hingegen zeigen eine schwach ausgeprägte positive Korrelation $\epsilon_{\mathrm{ff}} \propto K_{\mathrm{SGS}}$ an, die sich als Schwanz der tropfenförmig elongierten Verteilung zu kleinen $\epsilon_{\mathrm{ff}}$ und $K_{\mathrm{SGS}}$ hin äußert.

Am besten lassen sich die Unterschiede zwischen den einzelnen Modellen natürlich bei Betrachtung der Abhängigkeit der Kaltphasensternentstehungseffizienz $\epsilon_{\bmod }$ von der spezifischen SGS-Energie erkennen, wie es anhand von Abb. 6.7 möglich ist.

\section{PN11-Modellfamilie}

In der obersten Reihe von Abb. 6.7 ist zu sehen, dass alle Modelle der PN11-Familie einen ähnlichen Verlauf der Sternentstehungseffizienzen $\epsilon_{\bmod }$ mit der spezifischen SGS-Energie zeigen. Es ähnelt dem bereits in Abschnitt 4.3 beschriebenen Verhalten der Gleichgewichtssternentstehungseffizienz $\epsilon_{\mathrm{ff}}$ in Abhängigkeit von der Turbulenzstärke, dort ausgedrückt in $\mathcal{M}_{\Sigma}$, wobei die meiste Sternentstehung im vierten der dort beschriebenen Turbulenzregimes stattfindet (siehe Abb. 4.3). Letzteres ist nicht überraschend, denn Turbulenz ist in den Klumpen kalten Gases weit im Überschallbereich, da die thermische Energie gegenüber der turbulenten meist vernachlässigbar klein ist. Die Ähnlichkeit rührt von dem Umstand her, dass die thermische Energie für kleine $K_{\mathrm{SGS}}$ (oder auch kleine $\mathcal{M}_{\Sigma}$ bei den BS12-Gleichgewichtslösungen) einen entscheidenden Beitrag zum effektiven Druckgleichgewicht zwischen kalter und warmer Phase liefert. Dies gilt sowohl bei den Gleichgewichtslösungen im Fall geringer turbulenter Injektionsraten als auch für geringe instantane Werte von $K_{\mathrm{SGS}}$ in den Simulationen. 
In dem Bereich $10 \mathrm{~km}^{2} \mathrm{~s}^{-2} \lesssim K_{\mathrm{SGS}} \lesssim 10^{3} \mathrm{~km}^{2} \mathrm{~s}^{-2}$ bleibt $\epsilon_{\mathrm{PN} \text {-family fast konstant, wie }}$ es bereits in BS14 angemerkt wurde. Tatsächlich fällt $\epsilon_{\mathrm{PN}-\text { family }}$ über einen Bereich von mehr als zwei Größenordnungen in $K_{\mathrm{SGS}}$ nur um einen Faktor von $\sim 2$ ab. Der Abfall selbst ist ein Effekt des Anwachsens der kritischen Überdichte $\exp \left(x_{\mathrm{cr}}\right) \propto$ $\alpha_{\text {vir }} \mathcal{M}_{\mathrm{c}}^{2}$ mit $K_{\mathrm{SGS}}$. Während sich die Verwendung des mff-Freifallzeitkoeffizienten kaum auf das Verhalten von $\epsilon_{\mathrm{PN}-\mathrm{FK}-\mathrm{mff}}$ gegenüber dem von $\epsilon_{\mathrm{PN}-\mathrm{FK}}$ auswirkt, so hat doch die Variation des $\theta$-Parameters zwischen einerseits 'PN' $(\theta=0,5)$ und 'PN-FK' oder 'PN-FK-mff' andererseits $(\theta=1)$ den Effekt, dass die $\epsilon_{\mathrm{PN}}$ bei geringen $K_{\mathrm{SGS}}$ zwar auf gleichem Niveau startet, aber mit höheren $K_{\mathrm{SGS}}$ schneller abfällt als bei den anderen Modellen der PN11-Familie, was zu einer im Vergleich um einen Faktor von $5 \times \epsilon_{\mathrm{PN}}\left(K_{\mathrm{SGS}} \approx 10^{2} \mathrm{~km}^{2} \mathrm{~s}^{-1}\right) \approx \epsilon_{\mathrm{PN}-\mathrm{FK}}\left(K_{\mathrm{SGS}} \approx 10^{2} \mathrm{~km}^{2} \mathrm{~s}^{-1}\right)$ geringeren Kaltphasensternentstehungseffizienz führt. Dieser Unterschied sorgt für die klumpigere Scheibe in Programmlauf mit 'PN' im Vergleich mit der des 'PN-FK'-Laufs, da die Galaxie für einen stabilisierten Zustand etwa dieselbe Menge an stellarer Rückwirkung und damit auch Sternentstehung - benötigt, wofür die lokal höheren Dichten in der klumpigeren Scheibe sorgen, da so die geringeren $\epsilon_{\mathrm{PN}}$ ausgeglichen werden können.

\section{HC08-13-Modellfamilie}

Das Verhalten der Modelle der HC08-13-Familie mit $K_{\mathrm{SGS}}$ (wie in der zweiten Reihe von Abb. $6.7 \mathrm{zu}$ sehen) ist dem der PN11-Familie gewisserweise entgegengesetzt. So startet $\epsilon_{\mathrm{HC}-\text { family }}$ für kleine $K_{\mathrm{SGS}}$ bei mit relativ geringen Werten und steigt für größere $K_{\text {SGS }}$ stetig an, was durch das Absinken der kritischen Überdichte $x_{\mathrm{cr}} \propto \alpha_{\mathrm{vir}} \mathcal{M}_{\mathrm{c}}^{-2}$ bei steigendem $K_{\mathrm{SGS}}$ erklärt werden kann. Alle Modelle dieser Familie erreichen dabei in dem wegen der stellaren Rückwirkungen bevorzugten $K_{\mathrm{SGS}}$-Bereich für Sternentstehung in etwa $\epsilon_{\mathrm{HC}-\text { family }} \approx 0,1$, was für eine ähnlich aufgelockerte Gasscheibe wie beispielsweise im 'PN-FK'-Programmlauf sorgt. Während sich die Diagramme für die Modelle 'HC' und 'HC-FK' im Wesentlichen gleichen, zeigt das 'HC-FK-mff'Modell davon stark abweichendes Verhalten. So starten die beiden erstgenannten Modelle für kleine $K_{\mathrm{SGS}}$ mit $\epsilon_{\mathrm{HC}-\text { family }} \approx 0$. Mit steigendem $K_{\mathrm{SGS}}$ wächst $\epsilon_{\mathrm{HC} \text {-family }}$ schnell an, wobei dieser Anstieg mit wachsendem $K_{\mathrm{SGS}}$ immer weiter abflacht und ab $K_{\mathrm{SGS}} \approx 30 \mathrm{~km}^{2} \mathrm{~s}^{-2}$ in ein Potenzgesetz $\epsilon_{\mathrm{HC}-\text { family }} \propto K_{\mathrm{SGS}}^{1 / 2}$ übergeht. Eine Abweichung von diesem Potenzgesetz lässt sich für $K_{\mathrm{SGS}} \lesssim 30 \mathrm{~km}^{2} \mathrm{~s}^{-2}$ im Fall des 'HC-FKmff'-Modells nicht feststellen. Der Unterschied ist bedingt durch das Vernachlässigen des zweiten, additiven Terms in der Berechnung der kritischen Überdichte in diesem Fall $\left(\exp \left(x_{\mathrm{cr}, \mathrm{HC}-\mathrm{FK}-\mathrm{mff}}\right) \propto \alpha_{\mathrm{vir}} \mathcal{M}_{\mathrm{c}}^{-2}\right.$ anstatt von $\left.\exp \left(x_{\mathrm{cr}, \mathrm{HC}, \mathrm{FK}}\right) \propto \alpha_{\mathrm{vir}} \mathcal{M}_{\mathrm{c}}^{-2}+\alpha_{\mathrm{vir}}\right)$. Für das allen Modellen der HC08-13-Familie gemeinsame Potenzgesetzverhalten bei großen $K_{\mathrm{SGS}}$ ist also der Term $\propto \alpha_{\text {vir }} \mathcal{M}_{\mathrm{c}}^{-2}$ verantwortlich. Der Term $\propto \alpha_{\text {vir }}$ hingegen sorgt für den rapiden Abfall von $\epsilon_{\mathrm{HC}}$ und $\epsilon_{\mathrm{HC}-\mathrm{FK}}$, falls $K_{\mathrm{SGS}} \lesssim 30 \mathrm{~km}^{2} \mathrm{~s}^{-2}$ gilt. 


\section{KM05-Modellfamilie}

Die Modelle der KM05-Familie (siehe in der dritten Reihe von Diagrammen in Abb. 6.7) zeigen, wie bereits die der PN11-Familie, einen absteigenden Trend mit wachsendem $K_{\mathrm{SGS}}$, was daran liegt, dass sich die jeweiligen kritischen Überdichten die selbe Abhängigkeit $\left(\exp \left(x_{\mathrm{cr}}\right) \propto \alpha_{\text {vir }} \mathcal{M}_{\mathrm{c}}^{2}\right)$ teilen. Zwischen den Modellen 'PNFK-mff' und 'KM-FK-mff' besteht sogar abgesehen von unterschiedlichen Modellkoeffizienten kein formaler Unterschied, weshalb sich auch die entsprechenden Diagramme in Abb. 6.7 annähernd gleichen. Anhand eines Vergleiches der Diagramme von 'KM-FK' und 'KM-FK-mff' lässt sich leicht der Effekt der Verwendung eines Freifallzeitfaktors ablesen. Dieser steigert die Sternentstehungseffizienz, und zwar wegen der Abhängigkeit der Standardabweichung $\left(\sigma_{x} \propto \log \left(\mathcal{M}^{2}\right)\right)$ der log-normalen Dichte-pdf um so mehr, je turbulenter das Gas ist. Dies bewirkt einen weit weniger steilen Abfall von $\epsilon_{\mathrm{KM}-\mathrm{FK}-\mathrm{mff}}$ mit steigendem $K_{\mathrm{SGS}}{ }^{3}$ als es für $\epsilon_{\mathrm{KM}-\mathrm{FK}}$ ohne den Freifallzeitfaktor der Fall ist. Ähnlich wie bereits für das 'PN'-Modell festgestellt, liegt $\epsilon_{\mathrm{KM}-\mathrm{FK}}$ im relevanten Bereich $10 \mathrm{~km}^{2} \mathrm{~s}^{-2} \lesssim K_{\mathrm{SGS}} \lesssim 10^{3} \mathrm{~km}^{2} \mathrm{~s}^{-2}$ meist

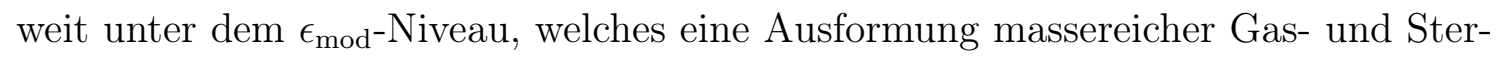
nenhaufen weitestgehend verhindern würde. Um die benötigte Menge von stellarer Rückwirkung zur Erreichung eines statistisch stabilen, sternbildenden Zustands der Gasscheibe zu erhalten, ist also lokal weit höher verdichtetes Gas notwendig. Dieses ist nur in massereichen Klumpen anzutreffen. Leider fällt $\epsilon_{\mathrm{KM}-\mathrm{FK}}$ mit steigendem $K_{\text {SGS }}$ relativ steil ab, was zur Folge hat, dass Sternentstehung gerade innerhalb der Klumpen weniger effizient ist, als es beim 'PN'-Modell der Fall wäre. Innerhalb dieser Klumpen ist $K_{\mathrm{SGS}}$ besonders groß, einerseits wegen des einfallenden Gases, andererseits wegen der lokal konzentrierten Stärke der stellaren Rückwirkung. Abgesehen davon ist der Effekt der stellaren Rückwirkung auf die Struktur der Gasscheibe um so geringer je massereicher der Gas- und Sternenklumpen bereits sind, da ein immer tieferes, lokales Gravitationspotential überwunden werden muss, bevor sich die injizierte Energie auf die Struktur der Scheibe auswirken kann. So kommt es bei der Verwendung des 'KM-FK'-Modells zwar zu späten Zeiten in der Simulation zu einer erhöhten globalen Sternentstehungsrate. Diese kann aber nicht das weitere Wachsen der massereichen Klumpen effektiv genug verlangsamen. Infolgedessen wird die Sternentstehung immer weiter angefacht, obwohl die lokale Kaltphaseneffizienz dabei immer weiter absinkt. Für kleine $K_{\mathrm{SGS}} \lesssim 10 \mathrm{~km}^{2} \mathrm{~s}^{-2}$ springt $\epsilon_{\mathrm{KM}-\mathrm{FK}}$ rasch auf einen nahezu konstanten Wert $\lesssim 1$, weil an diesem Punkt die kritische Überdichte kleiner wird als die mittlere Dichte des Kaltphasengases. ${ }^{4}$. Die Stelle, an der dieser

\footnotetext{
${ }^{3}$ Der bei Verwendung des mff-Freifallzeitfaktors auftauchende multiplikative Term $c$ aus Tabelle 6.1 ist für große $\mathcal{M}_{\mathrm{c}}$ wegen konstantem $e_{\mathrm{c}}$ annähernd proportional zu $K_{\mathrm{SGS}}: c \propto \mathcal{M}_{\mathrm{c}}^{2} \propto K_{\mathrm{SGS}}$.

${ }^{4}$ Der schnelle Umschlag resultiert aus dem Verhalten der Gauß'schen Fehlerfunktion erf $(z)$ um $z=0$ herum.
} 
Übergang stattfindet, wird maßgeblich durch den Modellparameter $\Phi_{x}$ bestimmt, so liegt er für das 'KM'-Modell $\left(\Phi_{x}=1,12\right)$ bei weit geringeren $K_{\mathrm{SGS}}$, die aber innerhalb der Simulationen nicht erreicht werden können. Das relativ große $\Phi_{x}=1,12$ sorgt beim 'KM'-Modell insgesamt für um einige Größenordnungen zu geringe $\epsilon_{\mathrm{KM}}$, als dass die der Sternentstehung nachfolgende stellare Rückwirkung die Gasscheibe ausreichend stabilisieren könnte.

\section{PHN12-Modell}

Das Diagramm in der unteren linken Ecke von Abb. 6.7 zeigt, dass die untere Einhüllende der $\epsilon_{\mathrm{PHN}}-$ Verteilung ein Verhalten zeigt, das auf eine implizite Abhängigkeit $\epsilon_{\mathrm{PHN}} \sim \exp \left(-K_{\mathrm{SGS}}^{2}\right)$ schließen lässt, obwohl im Argument der Exponentialfunktion nur $K_{\text {SGS }}^{1}$ explizit als Teil des Virialparameters $\alpha_{\text {vir }}$ eingeht. Ein weiterer, zusätzlich wirksamer Faktor $K_{\mathrm{SGS}}^{1}$ steckt in den impliziten Abhängigkeiten der Dichte $\rho_{\mathrm{c}, \mathrm{pa}}$ von $K_{\mathrm{SGS}}$ - und damit ebenfalls in der Kaltphasenklumpengröße $\ell_{\mathrm{c}}$. Diese impliziten Abhängigkeiten ergeben sich aus der Annahme eines Gleichgewichts des effektiven Druckes zwischen warmer und kalter Phase. Dass oberhalb dieser unteren Einhüllenden, besonders im Bereich $K_{\mathrm{SGS}} \gtrsim 10^{2} \mathrm{~km}^{2} \mathrm{~s}^{-2}$, sternbildendes Material anzutreffen ist, liegt vornehmlich an der bei fast allen anderen untersuchten Modellen stark unterdrückten Abhängigkeit von der Gasdichte $\rho$. Im Falle des PHN12-Modells ergibt sich für große $K_{\mathrm{SGS}}$ die Proportionalität $\log \left(\epsilon_{\mathrm{PHN}}\right) \propto K_{\mathrm{SGS}} / \rho$, wenn $\rho_{\mathrm{c}, \mathrm{pa}} \rightarrow \rho$ gilt. Generell ist die Sternentstehung bei Benutzung dieses Modells zu gering, um eine aufgelockerte Scheibe, wie sie etwa bei Verwendung von 'PN-FK' geformt wird, zu unterstützen. Es scheint aber plausibel, dass es nicht zu einem fortlaufenden Anwachsen der Sternentstehung kommt wie im Falle von 'KM' und 'KM-FK'. Aufgrund der günstigeren Abhängigkeit der Sternentstehungseffizienz $\epsilon_{\mathrm{PHN}}$ von der Gasdichte ist es - trotz geringem $\epsilon_{\mathrm{PHN}^{-N}}-\mathrm{Niveau}$ - möglich einen stabilen, sternbildenden $\mathrm{Zu}$ stand der Scheibe, mit einer globalen Sternentstehungsrate auf einem mit dem des 'PN-FK'-Programmlaufs vergleichbaren Niveau zu etablieren.

\subsection{Diskussion}

Eines der wichtigsten Ergebnisse aus den hier vorgestellten Simulationen ist, dass die globale Sternentstehungsrate $\dot{M}_{\mathrm{SF}}$ wie auch die Zeitskalen und Amplituden ihrer kurzzeitigen Variationen erstaunlich robust sind gegenüber der Variation des Modells für $\epsilon_{\text {mod }}$. Tatsächlich scheinen hauptsächlich die Anfangsbedingungen - also Verteilung und Gesamtmasse des Gases - sowie die Modellierung der stellaren Rückwirkung Verlauf und Niveau von $\dot{M}_{\mathrm{SF}}$ zu bestimmen. Auch der annähernd lineare Zusammenhang zwischen lokaler Sternentstehungsrate und molekularer Gasdichte bleibt unabhängig vom Sternentstehungsmodell bestehen. Ähnliches gilt da- 
her qualitativ auch für das Verhalten der lokalen Sternentstehungsrate gegenüber der Gesamtgasdichte, denn dieses wird primär durch den Übergang von atomarem zu molekularem Gas bestimmt. Die Wahl des Sternentstehungsmodells spielt eine untergeordnete Rolle, solange das spezielle Modell gewisse Mindestanforderungen erfüllt, auf die ich später noch eingehen möchte.

Sternentstehung findet schwerpunktmäßig in Gas statt, dessen spezifische turbulente SGS-Energie sich im Bereich $10 \mathrm{~km}^{2} \mathrm{~s}^{-2} \lesssim K_{\mathrm{SGS}} \lesssim 10^{3} \mathrm{~km}^{2} \mathrm{~s}^{-2}$ bewegt - egal, welches der untersuchten Modelle genutzt wird. Dieser Bereich ist durch die Rahmenbedingungen, die sich aus MIST und der Hydrodynamik im Rahmen von LES ergeben, bevorzugt, da einerseits die Bildung von molekularem Gas durch starke Turbulenz begünstigt wird, aber andererseits zu starke Turbulenz zur Expansion und damit zur Zerstörung einer sternbildenden Region führt.

Die resultierenden Gasscheiben in den verschiedenen Simulationen lassen sich anhand der Klumpigkeit ihres Aufbaus unterscheiden. Das eine Extrem bilden Gasscheiben von aufgelockerter Textur. Diese Scheiben werden über den gesamten sternbildenden Teil hinweg von kurzlebigen, spiralartigen, aber von Löchern durchsetzten Strukturen durchzogen. Das andere Extrem stellen Gasscheiben dar, die durch große (Sternen- und) Gasansammlungen dominiert werden. Lediglich die Gezeitenarme dieser massereichen Verklumpungen, die bei nahen Begegnungen oder Verschmelzungen derselben entstanden sind, durchbrechen die ansonsten mit heißem, stark verdünntem Gas ausgefüllte Scheibe zwischen den Klumpen. Verantwortlich dafür, wie klumpig die Gasscheibe wird, ist das Niveau der Kaltphasensternentstehungseffizienz $\epsilon_{\text {mod }}$ im Bereich von $10 \mathrm{~km}^{2} \mathrm{~s}^{-2} \lesssim K_{\mathrm{SGS}} \lesssim 10^{3} \mathrm{~km}^{2} \mathrm{~s}^{-2}$. Je geringer $\epsilon_{\text {mod }}$ ausfällt, desto klumpiger ist die Gasscheibe, und desto ausgeprägter ist der Schwanz der Dichteverteilung zu hohen Dichten hin.

Dies ist der Mechanismus, der dafür sorgt, dass die hier betrachteten Galaxiensimulationen meist eine vergleichbare globale Sternentstehungsrate aufweisen, obwohl die verschiedenen Sternentstehungsmodelle bisweilen sehr unterschiedliche $\epsilon_{\text {mod }}$ vorgeben. Letztere schlagen sich auch in den Sternentstehungszeitskalen bezüglich des kalten, molekularen Gases nieder. Im Falle von Modellen mit geringerem $\epsilon_{\text {mod }} \lesssim 0,1$ und sich daraus ergebenden längeren Sternentstehungszeitskalen ist das $\dot{\Sigma}_{\mathrm{s}}-\Sigma_{\mathrm{H}_{2}}$ Diagramm in Abb. 6.4 bis zu höheren Säulendichten hin bevölkert. Ausnahmen stellen die Modelle 'KM-FK' und 'KM' dar. Bei letzterem lässt sich auch nach 400 Ma Entwicklung nicht abschätzen, ob sich die Sternentstehungsrate vielleicht später noch auf dem Niveau der meisten anderen einregeln kann. Beide weisen allerdings ein für diesen Mechanismus ungünstiges Verhalten von $\epsilon_{\bmod } \propto K_{\mathrm{SGS}}^{\alpha_{\mathrm{KM}}}$ (mit $\left.\alpha_{\mathrm{KM}} \approx-1,2\right)$ auf. Sternentstehung wird hier bereits stark unterdrückt, bevor das sternbildende Gas ausreichend turbulenten Druck für eine Expansion aufbauen kann. Infolgedessen kann kaum Gas aus einem massereichen Klumpen ausgestoßen werden, 
sodass Sternentstehung sofort wieder einsetzt, sobald die von der stellaren Rückwirkung induzierte SGS-Turbulenz nachlässt.

Mangelnde Kaltphasensternentstehungseffizienz kann durch lokale Verdichtung des Gases ausgeglichen werden, um die von den übrigen Rahmenbedingungen vorgegebene mittlere globale Sternentstehungsrate zu erreichen. Diese Sternentstehungsrate bedingt die zur Stabilisierung der Gasscheibe erforderliche Injektion von thermischer und turbulenter Energie über stellare Rückwirkungsprozesse. In allen Simulationen mit physikalisch motivierten, analytischen Modellen, die einen Freifallzeitfaktor $f_{\mathrm{ff}, \text { mod }} \neq 1$ berücksichtigen, wie auch in der mit 'PHN'-Modell ist dieser Mechanismus wirksam. Bei den analytischen Modellen scheint $\epsilon_{\bmod }$ hauptsächlich eine Funktion nur einer Variablen zu sein, etwa der turbulenten SGS-Energie wie Abb. 6.7 suggeriert. Die nur geringe Streuung der in Abb. 6.7 gezeigten Verteilungen in $\epsilon_{\text {mod }}$-Richtung belegt dies. Die Modelle 'PHN', 'KM', 'KM-FK', sowie die Modelle der HC08-13-Familie (wenn auch weniger ausgeprägt) weisen hier eine nennenswerte Streuung auf, die auf eine subdominante Abhängigkeit von mindestens einer zweiten Variablen, wie der Gasdichte $\rho$, hindeutet. Aus den formalen Abhängigkeiten von den abgeleiteten Zustandsvariablen der kalten Phase - also $\ell_{\mathrm{c}}, \rho_{\mathrm{c}, \mathrm{pa}}$ und $\mathcal{M}_{\mathrm{c}}-$ wird ersichtlich, dass tatsächlich eine Variation von $K_{\mathrm{SGS}}$ den größten Einfluss auf $\epsilon_{\bmod }$ hat. Subdominante Abhängigkeiten von $\rho, \rho_{\mathrm{c}}$ oder $e_{\mathrm{w}}$ werden unter den Bedingungen, die durch die selbstregulierenden Eigenschaften von MIST innerhalb einer LES erzeugt werden, zusätzlich abgeschwächt, da sie effektiv untereinander aber auch mit $K_{\mathrm{SGS}}$ korreliert sind. Die Berücksichtigung eines Freifallzeitfaktors $f_{\mathrm{ff}, \bmod } \neq 1$ unterdrückt die Abhängigkeit von weiteren Variablen effektiv, wie beim Vergleich 'KM-FK' gegen 'KM-FK-mff' ersichtlich wird.

Die weiter oben angesprochenen Mindestanforderungen für ein Modell, mit dem ein stabiler, sternbildender Zustand der galaktischen Gasscheibe erreicht werden kann, sind erfüllt, wenn eine effektive Abhängigkeit $\epsilon_{\bmod } \propto K_{\mathrm{SGS}}^{\alpha_{\bmod }} \operatorname{mit} \alpha_{\bmod } \gtrsim-0,5$ gegeben ist. Diese stellt sicher, dass Sternentstehung solange stattfinden kann, bis der durch die stellare Rückwirkung aufgebaute Druck die Sternentstehungsregion auseinander treibt. Die Mindestanforderung kann aber offenbar auch durch eine positive Korrelation von $\epsilon_{\bmod }$ mit der Gasdichte erfüllt werden, wie es für 'PHN' der Fall ist.

Mit Hinblick auf die bei der Konstruktion von MIST benutzen Modellannahmen ${ }^{5}$ ist Sternentstehungsmodellen der Vorzug zu geben, die im für Sternentstehung optimalen Bereich $10 \mathrm{~km}^{2} \mathrm{~s}^{-2} \lesssim K_{\mathrm{SGS}} \lesssim 10^{3} \mathrm{~km}^{2} \mathrm{~s}^{-2}$ einen Wert von $\epsilon_{\text {mod }} \approx 0,1$ liefern, da dieser dem von Beobachtungen sternbildenden, dichten, molekularen Gases abge-

\footnotetext{
${ }^{5} \epsilon_{\text {mod }}$ bezieht sich ausschließlich auf das von $\mathrm{H}_{2}$-dissoziierender Strahlung abgeschirmte, dichte, kalte Gas, welches sich innerhalb klumpigen, turbulenten Ansammlungen kalten, neutralen Gases befindet.
} 
leiteten Wert entspricht (siehe beispielsweise Gao u. Solomon, 2004; Murray, 2011). Dieses optimale $K_{\mathrm{SGS}}$-Regime für Sternentstehung stimmt für alle betrachteten Modelle mit dem in Beobachtungen ermittelten turbulenten Regime aktiv sternbildender Regionen überein (siehe zum Beispiel Leroy u. a., 2008; Shetty u. a., 2012; Stilp u. a., 2013). Weitergehende, mit Beobachtungen vergleichende Aussagen zum effektiven Verhalten der lokalen Sternentstehungseffizienz mit der Stärke der Turbulenz in der betreffenden Region sind kaum zu treffen. Dies ist dem Umstand geschuldet, dass belastbare empirische Gesetzmäßigkeiten noch fehlen, die auf einer Vielzahl detaillierter Beobachtungen von dichtem, molekularem Gas in Sternentstehungsregionen basieren, welche speziell auch auf die Bestimmung ihrer Turbulenzeigenschaften abzielen. Im Besonderen ist eine differenzierende Beurteilung der von FK13 an Turbulenzsimulationen selbstgravitierenden Gases kalibrierten Modelle - abgesehen vom Spezialfall des 'KM-FK'-Modells - kaum möglich. Die Mitglieder dieser Modellgruppe sind allesamt mit den Beobachtungen von Gao u. Solomon (2004) sowie Murray (2011) vereinbar, weiterhin ergaben sich innerhalb dieser Gruppe keine signifikanten Unterschiede, was die Struktur der Gasscheibe, die resultierenden Sternentstehungsrelationen und auch die globalen Sternentstehungsraten betrifft. Das einzig aussagekräftige Unterscheidungsmerkmal, nämlich das effektive Verhalten von $\epsilon_{\bmod }$ mit $K_{\mathrm{SGS}}$ - beziehungsweise das der durch Beobachtungen erschließbaren Pendants, ist leider derzeit durch Beobachtungen nicht erforscht.

\subsection{Zusammenfassung}

In dieser Studie wurden vier verschiedene Modellfamilien - entwickelt und vorgestellt von PN11, KM05, HC08-13, beziehungsweise PHN12 - zur Abschätzung der Sternentstehungseffizienz in der kalten Phase im Rahmen von LES idealisierter, isolierter Scheibengalaxien unter Verwendung von MIST (BS14) untersucht. Hierzu wurde der in BS14 vorgestellte, mit 'ref' bezeichnete Programmlauf mit den jeweiligen Modellen und deren Variationen nach FK13 (siehe Tabelle 6.1 für eine Auflistung aller untersuchten Modelle) ab einem Simulationsalter von 1 Ga um 0,4 Ga fortentwickelt, um möglichst zu statistisch stationären Konfigurationen der sternbildenden Galaxienscheiben zu gelangen. Die Nutzung einer bereits vorentwickelten Scheibe soll unter Anderem die Effekte weitestgehend minimieren, die sich zwangsläufig aus dem kontinuierlichen Gasverbrauch durch Sternbildung oder aus der allmählichen Anreicherung des Gases mit schweren Elementen durch Auswürfe sterbender, massereicher Sterne ergeben. Die Struktur von MIST in Verbindung mit dem SGS-Modell von SF11 bietet die einmalige Gelegenheit, die für die verschiedenen Modelle relevanten Größen $\alpha_{\text {vir }}$ und $\mathcal{M}_{\mathrm{c}}$, sowie die mittlere Dichte $\rho_{\mathrm{c}, \mathrm{pa}}$ innerhalb der Klumpen kalten Gases mit Ausdehnung $\ell_{\mathrm{c}}$ auf Basis des lokalen, turbulenten Mehrphasenzu- 
stands zu bestimmen. Insgesamt habe ich in diesem Zusammenhang zehn Läufe des von mir speziell für den Zweck solcher Simulationen angepassten Programmcodes Nyx (Almgren u. a., 2013) durchgeführt.

Beim Vergleich der Simulationsergebnisse stellte sich heraus, dass sich die mittlere, globale Sternentstehungsrate weitestgehend unabhängig vom jeweils genutzten Sternentstehungsmodell für das molekulare Gas in der kalten Phase auf einem gewissen Niveau $\dot{M}_{\mathrm{SF}} \approx 2,5 \mathrm{M}_{\odot} \mathrm{a}^{-1}$ einpegelte. Zur Stabilisation der Gasscheibe wird eine bestimmte Energiefreisetzung innerhalb derselben benötigt, die lediglich von den globalen Eigenschaften des Galaxiensetups abhängt. Um diese Energieinjektionsrate zu erreichen wird unter der von mir benutzen Modellierung der stellaren Rückwirkung eben die genannte globale Sternentstehungsrate benötigt. Liefert ein bestimmtes Modell eine geringere Sternentstehungseffizienz, so wird dieser Mangel durch Veränderung der Struktur der Gasscheibe ausgeglichen, indem hoch dichte Gasklumpen ausgebildet werden. Die Scheibe wird unter diesen Umständen also klumpiger. Im entgegengesetzten Fall stellt sich eine aufgelockertere Struktur der Scheibe ein.

Der für Sternentstehung optimale Bereich der SGS-Geschwindigkeitsdispersion $\sigma_{\mathrm{SGS}}$ (auf Skalen von $\sim 30$ pc) liegt unabhängig vom Sternentstehungsmodell zwischen $3 \mathrm{~km} \mathrm{~s}^{-1} \lesssim \sigma_{\mathrm{SGS}} \lesssim 30 \mathrm{~km} \mathrm{~s}^{-1}$, was gut mit Beobachtungen übereinstimmt (Shetty u. a., 2012). Dieser Bereich ist auf der einen Seite durch die Turbulenzverstärkung der chemischen Reaktionsraten zur Bildung von molekularem Wasserstoff begrenzt, auf der anderen Seite durch die von stellarer Rückwirkung angetriebene Zerstörung sternbildender Regionen.

Beobachtungen - beispielsweise von Gao u. Solomon (2004) oder Murray (2011) legen nahe, Modelle zu bevorzugen, die Effizienzen in der Größenordnung $\epsilon_{\bmod } \sim 0,1$ liefern. Unter Verwendung solcher Modelle ergeben sich aufgelockerte Gasscheiben. Dies kann prinzipiell für alle Modelle durch geeignete Wahl der Modellkoeffizienten erreicht werden, wie die von FK13 an getriebenen Turbulenzsimulationen kalibrierten Modellvariationen belegen - mit Ausnahme von 'KM-FK', welchem es dafür effektiv an einem Freifallzeitfaktor fehlt. 'PHN' wurde zwar als formale Anpassung an Daten ähnlicher Simulationen wie der von FK13 gewonnen, dennoch erweist sich dieses Modell in meiner Simulation mit MIST als weniger geeignet. Möglicherweise liegt dies daran, dass die zugrunde liegenden Simulationen ausschließlich solenoidales Treiben berücksichtigten, was mit den Bedingungen, die für das kalte Gas in MIST gelten, nicht vereinbar ist.

Nicht nur weil die in dieser Studie vorgestellten Simulationen keine Magnetfelder berücksichtigen, lässt sich leider anhand dieser Studie kaum weiter differenzieren, welche der Annahmen, die bei der Konstruktion der erfolgreichen Modelle getroffen wurden, bei real stattfindender Sternentstehung tatsächlich erfüllt sind. Es feh- 
len schlicht beobachtungsgestützte, abgesicherte Erkenntnisse hierzu, was nicht nur wegen der erforderlichen räumlichen und spektralen Auflösung oder der geringen Anzahl nahe gelegener Beobachtungsziele schwierig ist. Außerdem dürften sich die Rahmenbedingungen der möglichen Beobachtungsziele, denen die Sternentstehung dort unterliegt, weitestgehend gleichen, da sie sich in der Milchstraße oder ihrer unmittelbaren Umgebung befinden müssen. Während die im Rahmen von Beobachtungen gemessenen Geschwindigkeitsdispersionen dem aktuellen, turbulenten Zustand des Gases entsprechen, sind gemessene Sternentstehungsraten immer als ein Mittel über die letzten paar Millionen Jahre zu verstehen, was die empirische Ermittlung des Zusammenhangs zwischen Sternentstehung und Turbulenz zusätzlich erschwert. So sind weitere Modelle denkbar, die sich zwar in Details der Turbulenz- und/oder Dichteabhängigkeit unterscheiden, aber ansonsten alle zu kaum unterscheidbaren Ergebnissen führen.

Diese Studie legt nahe, dass weder die globale Sternentstehungsrate noch globale Sternentstehungsrelationen geeignete diagnostische Werkzeuge sind, um die Gültigkeit verschiedener effektiver Modelle zur Abschätzung der Sternentstehungseffizienz zu prüfen. Aber auch der Mittelwert der Sternentstehungseffizienz innerhalb dichten, kalten, molekularen Gases ist nicht unbedingt ein adäquates Mittel, da dieser stark von den jeweiligen Modellkoeffizienten abhängt, bezüglich derer ein großes Maß an Unsicherheit herrscht.

Eine Abwandlung oder Erweiterung der Modellierung der stellaren Rückwirkung im Rahmen von MIST, wie in BS14 vorgeschlagen, wird gegebenenfalls die zur Stabilisierung der galaktischen Gasscheibe erforderliche Sternentstehungsrate verändern. Die übrigen Ergebnisse dieser Studie dürften Bestand haben. 


\section{Kapitel 7}

\section{Zusammenfassung und Ausblick}

Im Rahmen dieses umfangreichen Promotionsprojektes wurde ein physikalisch motiviertes Modell für das sternbildende, turbulente ISM (für Skalen von wenigen zehn Parsec) entwickelt und als MIST in den kosmologischen Hydrodynamikcode Nyx implementiert. Hierzu wurden verschiedene Ansätze zur Beschreibung einzelner Aspekte des ISM zusammengebracht, um sie zu einen effektiven, dynamischen Modell zu verbinden, das in Form eines Satzes gekoppelter partieller Differentialgleichungen mit einigen Nebenbedingungen von mir formuliert wurde. Während sich andere für die Simulation einzelner Galaxien entworfene Modelle - auch das von Agertz u. a. (2013) - hauptsächlich auf die stellare Rückwirkung konzentrieren, bleiben diese meist relativ einfach, was die Modellierung des ISM und der darin ablaufenden Sternentstehung anbetrifft (keine Berücksichtigung der Multiphasennatur sowie der kleinskaligen Turbulenz des ISM, konstante Sternentstehungseffizienz oberhalb einer Mindestdichte). Murante u. a. (2010) zum Beispiel verwenden ein einfaches Zweiphasenmodell des ISM in ihren Simulationen. Dieses beschränkt sich allerdings auf eine konstante Sternentstehungseffizienz und verzichtet darauf, kleinskaliger Turbulenz Rechnung zu tragen. Renaud u.a. (2013) beispielsweise passen ihre Sternentstehungseffizienz explizit so an, dass sich in ihrer Simulation die gewünschte Sternentstehungsrate ergibt.

Die besondere Stärke von MIST liegt in der innovativen Einbindung der kleinskaligen Turbulenz als dynamische Einfluss- und Verlaufsgröße sowie der Anbindung an das SGS-Turbulenzmodell von SF11. Einige der bei der Konstruktion des Modells benutzten Annahmen sind zwar plausibel, aber nicht durch Beobachtungen abgesichert. Es handelt sich hierbei um die Hypothesen, dass im ISM zwischen kalten Wolken und umgebendem warmem Medium ein Gleichgewicht des effektiven Druckes herrscht, und dass die typische Ausdehnung dieser Wolken durch ihre Jeanslänge abgeschätzt werden kann. Diese Annahmen stellen sicher, dass die behandelten Phasen abgesehen von den modellierten Austauschbeziehungen hydrodynamisch und (global) gravitativ stabil sind. Zumindest für die Annahme des Druckgleichgewichts gibt 
es Hinweise von Beobachterseite (zum Beispiel Hughes u. a., 2013a), dass diese Annahme gerechtfertigt ist.

Sowohl MIST (BS14) als auch sein semi-analytischer Vorgänger (BS12) sind in der Lage erfolgreich Beobachtungsergebnisse zu reproduzieren, die entsprechenden, bereits veröffentlichten Journalartikel sind auch im Anhang (A.1 beziehungsweise A.2) $\mathrm{zu}$ finden.

Die wichtigsten Eckpunkte des BS12-Modells sollen nun kurz aufgelistet werden:

- Aufteilung des ISM in zwei Phasen eine kalte, klumpige und eine diffuse, warme

- Gleichgewicht des effektiven Druckes zwischen den Phasen auf Skalen der typischen Abmessung eines Kaltphasenklumpens

- Massen und Energieaustausch zwischen den Phasen aufgrund verschiedener Prozesse: radiatives Kühlen, turbulente Dissipation, photoelektrisches Heizen, Lyman-Kontinuum Strahlung junger Sterne, SN-Ereignisse, Sternentstehung

- dynamische Berechnung der Sternentstehungsrate aus den turbulenten Zustand des Kaltphasengases und dem mittels eines Strømgrenansatzes bestimmten Anteil abgeschirmten, molekularen Gases in der kalten Phase

- Rückwirkung der Sterne auf das Gas über SN und Lyman-Kontinuumsstrahlung gemäß ihres Alters und ihrer Metallizität

- Turbulenzstärke als dynamische Verlaufsvariable, die in fast alle modellierten Prozesse eingreift, und sich aus externen Quellen (turbulente Kaskade) und internen Quellen (SN-Explosionen und Phasenseparation) speist

- modellierter Übergang zwischen neutralem und molekularem Gas vergleichbar mit beobachtetem

- Reproduktion empirischer Sternentstehungsrelationen sowohl für die Gesamtgasdichte $\left(\alpha_{\mathrm{KS}} \approx 1,5\right)$ als auch für die molekulare Dichte (linear, $\left.\alpha_{\mathrm{KS}} \approx 1\right)$ in Gleichgewichtslösungen.

MIST besitzt einige Eigenschaften, die es vom BS12-Modell unterscheiden:

- eine zusätzliche, innerhalb der warmen Phase angesiedelte, heiße SN-Gasphase, die nicht radiativ kühlt, aber langsam der restlichen warmen Phase untergemischt wird

- Auslagerung der stellaren Prozesse an kollisionsfreie Partikel.

- Anbindung an das SGS-Modell für kleinskalige Turbulenz in LES 
Die Implementierung in einen Hydrodynamikprogrammcode gestaltete sich aufwändiger als erwartet. Erst stellte sich heraus, dass der anfänglich genutzte Code ENZO nicht den Anforderungen meiner Problemstellung entsprach, da es dessen Hydrolöser an Stabilität und Robustheit mangelte. Der Wechsel zum Code Nyx brachte andere Unwägbarkeiten mit sich. Denn dieser war damals noch unfertig. So habe ich neben der Implementierung der eigentlichen MIST-Routinen eigenständig Teile der Code-Infrastruktur - zum Beispiel zur Behandlung stellarer Partikel - erstellt und bei der Implementierung des SGS-Modells mitgewirkt (siehe SA14).

Die schließlich durchgeführten LES von idealisierten IDG zeigten die Leistungsfähigkeit von MIST im Zusammenwirken mit dem SGS-Modell von SF11, indem viele Beobachtungsergebnisse gleichzeitig erfolgreich reproduziert werden konnten:

- realistische globale Sternentstehungsraten von einigen $\mathrm{M}_{\odot} a^{-1}$

- mittlere Sternentstehungseffizienz bezüglich der Gesamtgasdichte $\epsilon_{\mathrm{ff}} \lesssim 0.01$

- Sternentstehungseffizienz $\epsilon_{\mathrm{ff}}$ in besonders dichten Regionen stark erhöht

- galaktische Sternentstehungszeitskala $\tau_{\text {dep,gal }}=0,3 \ldots 1$ Ga (vergleichbar mit beobachteten (extrem) gasreichen Spiralgalaxien)

- lineare KS-Relation für das dichte molekulare Gas $\dot{\Sigma}_{\mathrm{s}} \propto \Sigma_{\mathrm{H}_{2}}$

- Lebensdauer einer aktiven Sternentstehungsregion von 10...30 Ma

- mittlere Geschwindigkeitsdispersion in Sternentstehungsgebieten im Bereich von $10 \mathrm{~km} \mathrm{~s}^{-1}$

- Sternentstehungszeitskala bezüglich des abgeschirmten, molekularen Wolken $\tau_{\text {dep,gal }}=40 \ldots 100 \mathrm{Ma}$.

- turbulente galaktische Winde heißen, metallangereicherten Gases, die die Scheibe mit $200 \ldots 1000 \mathrm{~km} \mathrm{~s}^{-1}$ verlassen

Hervorzuheben ist die lineare KS-Relation für das abgeschirmte, molekulare Gas, die so nicht im Modell festgelegt wurde, sondern durch die interne Selbstregulation des Modells zustande kommt. Als besonders vorteilhaft hat sich die Umsetzung der stellaren Rückwirkung herausgestellt. Durch die Aufteilung der Injektion der SN-Energie in einen turbulenten und einen thermischen Anteil sowie die Einführung der auf der Zeitskala von 1 Ma zerfallenden SN-Gasphase konnte das sogenannte 'over-cooling'-Problem umgangen werden. Andere Forscher müssen in ihren Simulationen das Kühlen lokal künstlich für einen Zeitraum von einigen 10 Ma abschalten, wenn dort SN stattfanden. Ansonsten würde die SN-Energie sofort wieder durch Abstrahlung verloren gehen, ohne sich nennenswert auf das Gas auszuwirken. Dass 
die SN-Energie länger im Gas verweilt, was die Evaporation der Sternentstehungsgebiete und die Entstehung von SN-Blasen bewirkt, kann in meinen Simulationen mit wesentlich geringerem Eingriff in die Physik des Galaxienmodells bewirkt werden, als es ohne SGS-Modell und turbulente stellare Rückwirkung der Fall wäre.

Zusätzlich zu den Veröffentlichungen BS12 und BS14 habe ich eine noch nicht publizierte Untersuchung über den Einfluß verschiedener Modelle (KM05; HC0813; PN11; PHN12 auch in den Parametrisierungen von FK13) zur Abschätzung der Sternentstehungsrate im kalten Gas angestellt. Dabei zeigten sich globale Sternentstehungsrate, die Art der gefundenen KS-Relationen und das für Sternentstehung optimale Turbulenzregime als bemerkenswert robust gegenüber dieser Variation. Diese werden maßgeblich durch das Galaxiensetup und die verwendeten Modelle für die Bestimmung des molekularen Gasanteils und der stellaren Rückwirkung bestimmt, nicht durch das Kaltphasensternentstehungsmodell. Eine Anpassung der Effizienz, wie sie zum Beispiel Renaud u.a. (2013) vornahmen, um eine gewünschte Sternentstehungsrate zu erhalten ist also bei Anwendung von MIST - wenn überhaupt - nur begrenzt möglich. Massiven Einfluss hat die Kaltphasenstenenstehungseffizienz auf die Struktur der Gasscheibe. So ist die Scheibe aufgelockerter und besitzt unvollständig ausgebildete Spiralarme, wenn die Kaltphasenstenenstehungseffizienz hoch ( $\sim 0,1$ im relevanten Turbulenzregime) ist. Falls die Effizienz geringer ausfällt, ist die Gasscheibe tendenziell klumpiger und stärker von massiven Sternhaufen geprägt. Vergleiche mit Beobachungsergebnissen weisen dahin, dass höhere Effizienzen $\sim 0,1$ eher realistisch sind als geringere. Weiterhin konnte für das neutrale Gas eine KS-Relation mit $\alpha_{\mathrm{KS}} \approx 2$ für hohe Dichten festgestellt werden, was in derartig gasreichen Galaxien wie den von mir simulierten plausibel erscheint.

Auf dem Weg eine noch realistischere Modellierung des ISMs in MIST zu erreichen, wäre die Berücksichtigung von weiteren stellaren Rückwirkungsprozessen vorteilhaft. Hier sind insbesondere stellare Winde massereicher, junger Sterne sowie SN vom Typ Ia zu erwähnen. Dadurch würde nämlich unter Umständen die Bildung von super-massereichen Sternhaufen verhindert, die zwar selten auftreten, aber ein Artefakt in den bisherigen Simulationen darstellen könnten. Stellare Winde würden bewirken, dass Sternhaufen weniger stark gravitativ gebunden sind, weil während einer frühen Entwicklungsphase einer Sternentstehungsregion gebildete Sterne eine höhere Geschwindigkeitsdispersionen aufwiesen. SN vom Typ Ia könnten die Akkretion von Gas auf bereits bestehende Sternhaufen erschweren, sodass diese nicht mehr zu derart massiven Gebilden anwachsen können.

Als zukünftige Fortsetzungen dieses Projekts bietet es sich an, andere Typen von Galaxien zu untersuchen. Die im früheren Universum zahlreicheren Zwerggalaxien sind vermutlich dafür verantwortlich, dass sich auch weitab von Galaxien Beimengun- 
gen von Metallen im intergalaktischen Medium befinden. Ihr Gravitationspotential ist nämlich weniger tief, als das 'normaler' Galaxien, sodass durch SN mit Metallen angereichertes Gas aus Zwerggalaxien leichter und in Relation zur Masse einer Zwerggalaxie in größerer Menge entkommen kann. LES mit MIST von idealisierten Zwerggalaxien wären in diesem Zusammenhang ein lohnendes Ziel, nicht nur um die Entwicklung ihres ISM und ihrer Sternenpopulationen zu erforschen, sondern auch um die abgeblasenen galaktischen Winde genauer zu untersuchen. Studien unter Verwendung von MIST zu extern gestörten Scheibengalaxien wären interessant. Die Störer stehen zwar stellvertretend für interagierende Galaxien im Umfeld der simulierten Galaxie, müssten aber nicht explizit als solche berücksichtigt werden. Ihre gravitative Wirkung allein wäre ausreichend, solange sie sich weit genug entfernt von der eigentlich untersuchten Galaxie befinden. So könnte die Auswirkung externer Störquellen auf Struktur und Morphologie von Gas- und Sternenscheiben sowie auf die Sternentstehung im Detail erforscht werden. Abgesehen davon sind auch Simulationen der Entwicklung von Scheiben- und Zwerggalaxien innerhalb eines realistischeren Umfeldes wünschenswert, was auch Verschmelzungen von Galaxien mit einschließst. Das Mittel der Wahl hierfür sind kosmologische LES mit MIST. Im diesem Fall, da man sich für die Entwicklungen von lediglich einer Galaxie inklusive ihrer Verschmelzungsgeschichte interessiert, sind sogenannte 'zoom-in'Simulationen angebracht. Im Rahmen dieser wird ein großes physikalisches Volumen relativ grob modelliert, während ein ausgewählter, kleiner Ausschnitt davon mittels AMR mit wesentlich höherer numerischer Auflösung simuliert wird. Allerdings ist für die meisten dieser Zwecke eine Erweiterung von MIST notwendig. Für eine möglichst realistische Modellierung von Zwerggalaxien und für die kosmologischen 'zoom-in'Simulationen wird eine explizite Behandlung der relevanten, chemischen Prozesse, die bei Bildung und Zerstörung von molekularem Wasserstoff beteiligt sind. Die bisherige Formulierung über den Strømgrenansatz ist nicht ausreichend, da diese nur für bereits metallangereichertes Gas mit $Z \geq 0,1 Z_{\odot}$ verlässlich ist, nicht aber für metallärmeres (oder im Extremfall primordiales) Gas. Außerdem würde die explizite Modellierung der Wasserstoffchemie in den Gasphasen inklusive des Austauschs zwischen ihnen auch die Möglichkeit bieten, bei der Analyse der Simulationsresultate Vergleichbarkeit mit Beobachtungen von weniger dichtem, nur teilweise von dissoziierender Strahlung abgeschirmtem Gas (etwa aus Beobachtungen im Licht von Linienübergangen des Kohlenmonoxidmoleküls) herzustellen.

MIST ist in Verbindung mit einem dynamischen SGS-Modell wie dem von SF11 ein wirksames und vielversprechendes Werkzeug zur Erforschung von Galaxien, ihrem ISM sowie ihrer Umgebungen mittels Simulationen. Es kann in zukünftigen Anwendungen weitere wichtige Beiträge zum Verständnis der beteiligten Prozesse sowie den beobachteten Strukturen und Zusammenhängen liefern. 


\section{What being a scientist is all about.}

Farnsworth: These are the dark matter engines I invented.

They allow my starship to travel between galaxies in mere hours.

Cubert: $\quad$ That's impossible. You can't go faster than the speed of light.

Farnsworth: Of course not. That's why scientists increased the speed of light in 2208.

Cubert: $\quad$ Also impossible.

Farnsworth: And what makes my engines truly remarkable is the afterburner which delivers $200 \%$ fuel efficiency.

Cubert: That's especially impossible.

Farnsworth: Not at all. It's very simple.

Cubert: $\quad$ Then explain it.

Farnsworth: Now that's impossible.

It came to me in a dream and I forgot it in another dream.

Cubert: Your explanations are pure weapons-grade bollock-nium.

It's all impossible.

Farnsworth: Nothing is impossible.

Not if you can imagine it. That's what being is a scientist is all about.

Cubert: No, that's what being a magical elf is all about.

- Dialogfragment aus der Episode 'A Clone of My Own' der Fernsehserie 'Futurama'. 


\section{Literaturverzeichnis}

[Agertz u. a. 2013] Agertz, O. ; Kravtsov, A. V. ; Leitner, S. N. ; Gnedin, N. Y.: Toward a Complete Accounting of Energy and Momentum from Stellar Feedback in Galaxy Formation Simulations. In: Astrophysical Journal 770 (2013), Juni, S. 25. http://dx.doi.org/10.1088/0004-637X/770/1/25. - DOI $10.1088 / 0004-637 \mathrm{X} / 770 / 1 / 25$

[Agertz u. a. 2009] Agertz, O. ; Lake, G. ; Teyssier, R. ; Moore, B. ; Mayer, L. ; RomeO, A. B.: Large-scale galactic turbulence: can self-gravity drive the observed HI velocity dispersions? In: Monthly Notices of the Royal Astronomical Society 392 (2009), S. 294-308. http://dx.doi.org/10.1111/j.1365-2966.2008.14043.x. - DOI 10.1111/j.1365-2966.2008.14043.x

[Agertz u. a. 2011] Agertz, O. ; Teyssier, R. ; Moore, B.: The formation of disc galaxies in a $\Lambda \mathrm{CDM}$ universe. In: Monthly Notices of the Royal Astronomical Society 410 (2011), Januar, S. 1391-1408. http://dx.doi.org/10.1111/j. 1365-2966.2010.17530.x. - DOI 10.1111/j.1365-2966.2010.17530.x

[Almgren u. a. 2013] Almgren, A. S. ; Bell, J. B. ; Lijewski, M. J. ; Lukić, Z. ; VAn Andel, E.: Nyx: A Massively Parallel AMR Code for Computational Cosmology. In: Astrophysical Journal 765 (2013), März, S. 39. http://dx.doi. org/10.1088/0004-637X/765/1/39. - DOI 10.1088/0004-637X/765/1/39

[Alongi u. a. 1993] Alongi, M. ; Bertelli, G. ; Bressan, A. ; Chiosi, C. ; FAgotto, F. ; Greggio, L. ; NASI, E.: Evolutionary sequences of stellar models with semiconvection and convective overshoot. I - $\mathrm{Z}=0.008$. In: Astronomy and Astrophysics Supplement 97 (1993), März, S. 851-871

[Behrens u. Braun 2014] Behrens, C. ; Braun, H.: Inclination Dependence of Lyman-Alpha Properties in a Turbulent Disk Galaxy. In: ArXiv e-prints (2014), Oktober

[Benincasa u. a. 2013] Benincasa, S. M. ; Tasker, E. J. ; Pudritz, R. E. ; WAdsley, J.: Giant Molecular Cloud Formation in Disk Galaxies: Characterizing Simulated versus Observed Cloud Catalogs. In: Astrophysical Journal 776 
(2013), Oktober, S. 23. http://dx.doi.org/10.1088/0004-637X/776/1/23. DOI $10.1088 / 0004-637 \mathrm{X} / 776 / 1 / 23$

[Bertelli u.a. 1994] Bertelli, G. ; Bressan, A. ; Chiosi, C. ; Fagotto, F. ; NASI, E.: Theoretical isochrones from models with new radiative opacities. In: Astronomy and Astrophysics Supplement 106 (1994), August, S. 275-302

[Bigiel u.a. 2011] Bigiel, F. ; Leroy, A. K. ; Walter, F. ; Brinks, E. ; De Blok, W. J. G. ; Kramer, C. ; Rix, H. W. ; Schruba, A. ; Schuster, K.F. ; Usero, A. ; Wiesemeyer, H. W.: A Constant Molecular Gas Depletion Time in Nearby Disk Galaxies. In: The Astrophysical Journal Letters 730 (2011), April, S. L13+. http://dx.doi.org/10.1088/2041-8205/730/2/L13. - DOI 10.1088/2041-8205/730/2/L13

[Binney u. Merrifield 1998] Binney, J. ; Merrifield, M.: Galactic Astronomy. Princeton University Press, 1998

[Blitz u. a. 2007] Blitz, L. ; Fukui, Y. ; Kawamura, A. ; Leroy, A. ; Mizuno, N. ; Rosolowsky, E.: Giant Molecular Clouds in Local Group Galaxies. In: Protostars and Planets V (2007), S. 81-96

[Bodenheimer u. a. 2006] Bodenheimer, P. ; Laughlin, G. P. ; RozyczkA, M. ; Yorke, H. W.: Numerical Methods in Astrophysics: An Introduction (Series in Astronomy and Astrophysics). CRC Press, 2006

[Bonnor 1956] Bonnor, W. B.: Boyle's Law and gravitational instability. In: Monthly Notices of the Royal Astronomical Society 116 (1956), S. 351

[Booth u. a. 2013] Booth, C. M. ; Agertz, O. ; Kravtsov, A. V. ; Gnedin, N. Y.: Simulations of Disk Galaxies with Cosmic Ray Driven Galactic Winds. In: The Astrophysical Journal Letters 777 (2013), November, S. L16. http://dx.doi. org/10.1088/2041-8205/777/1/L16. - DOI 10.1088/2041-8205/777/1/L16

[Braun u. Schmidt 2012] Braun, H. ; Schmidt, W.: A semi-analytic model of the turbulent multi-phase interstellar medium. In: Monthly Notices of the Royal Astronomical Society 421 (2012), April, S. 1838-1860. http://dx.doi.org/10. 1111/j.1365-2966.2011.19889.x. - DOI 10.1111/j.1365-2966.2011.19889.x

[Braun u.a. 2014] Braun, H. ; Schmidt, W. ; Niemeyer, J. C. ; Almgren, A. S.: Large-eddy simulations of isolated disc galaxies with thermal and turbulent feedback. In: Monthly Notices of the Royal Astronomical Society 442 (2014), August, S. 3407-3426. http://dx.doi.org/10.1093/mnras/stu1119. - DOI $10.1093 / \mathrm{mnras} / \mathrm{stu} 1119$ 
[Bressan u. a. 1993] Bressan, A. ; Fagotto, F. ; Bertelli, G. ; Chiosi, C.: Evolutionary sequences of stellar models with new radiative opacities. II - $\mathrm{Z}=$ 0.02. In: Astronomy and Astrophysics Supplement 100 (1993), September, S. 647664

[Bryan u. a. 2014] Bryan, G. L. ; Norman, M. L. ; O’Shea, B. W. ; Abel, T. ; Wise, J. H. ; Turk, M. J. ; Reynolds, D. R. ; Collins, D. C. ; Wang, P. ; Skillman, S. W. ; Smith, B. ; Harkness, R. P. ; Bordner, J. ; Kim, J.-h. ; Kuhlen, M. ; Xu, H. ; Goldbaum, N. ; Hummels, C. ; Kritsuk, A. G. ; Tasker, E. ; Skory, S. ; Simpson, C. M. ; Hahn, O. ; Oishi, J. S. ; So, G. C. ; Zhao, F. ; Cen, R. ; Li, Y. ; Enzo Collaboration: EnZO: An Adaptive Mesh Refinement Code for Astrophysics. In: Astrophysical Journal Supplement 211 (2014), April, S. 19. http://dx.doi.org/10.1088/0067-0049/211/2/19. DOI $10.1088 / 0067-0049 / 211 / 2 / 19$

[Chabrier 2001] Chabrier, G.: The Galactic Disk Mass Budget. I. Stellar Mass Function and Density. In: Astrophysical Journal 554 (2001), Juni, S. 1274-1281. http://dx.doi.org/10.1086/321401. - DOI 10.1086/321401

[Clarke u. Carswell 2014] Clarke, C. ; Carswell, B.: Principles of Astrophysical Fluid Dynamics. Cambridge University Press, 2014

[Daddi u. a. 2010] Daddi, E. ; Bournaud, F. ; Walter, F. ; Dannerbauer, H. ; Carilli, C. L. ; Dickinson, M. ; Elbaz, D. ; Morrison, G. E. ; Riechers, D. ; Onodera, M. ; Salmi, F. ; Krips, M. ; Stern, D.: Very High Gas Fractions and Extended Gas Reservoirs in $\mathrm{z}=1.5$ Disk Galaxies. In: Astrophysical Journal 713 (2010), April, S. 686-707. http://dx.doi.org/10.1088/0004-637X/713/1/686. - DOI 10.1088/0004-637X/713/1/686

[Dobbs u. Pringle 2013] DobBs, C. L. ; Pringle, J. E.: The exciting lives of giant molecular clouds. In: Monthly Notices of the Royal Astronomical Society 432 (2013), Juni, S. 653-667. http://dx.doi.org/10.1093/mnras/stt508. - DOI $10.1093 / \mathrm{mnras} / \mathrm{stt} 508$

[Ebert 1955] EBERT, R.: Über die Verdichtung von H I-Gebieten. Mit 5 Textabbildungen. In: Zeitschrift für Astrophysik 37 (1955), S. 217

[Evans u. a. 2009] Evans, N. J. II ; Dunham, M. M. ; Jørgensen, J. K. ; Enoch, M. L. ; Merín, B. ; van Dishoeck, E. F. ; COAuthors, 12: The Spitzer c2d Legacy Results: Star-Formation Rates and Efficiencies; Evolution and Lifetimes. In: Astrophysical Journal Supplement 181 (2009), April, S. 321-350. http://dx. doi .org/10.1088/0067-0049/181/2/321 - DOI 10.1088/0067-0049/181/2/321 
[Federrath 2013] FederRATh, C.: On the universality of supersonic turbulence. In: Monthly Notices of the Royal Astronomical Society 436 (2013), Dezember, S. 1245-1257. http://dx.doi.org/10.1093/mnras/stt1644. - DOI $10.1093 / \mathrm{mnras} / \mathrm{stt} 1644$

[Federrath u. Klessen 2013] Federrath, C. ; Klessen, R. S.: On the Star Formation Efficiency of Turbulent Magnetized Clouds. In: Astrophysical Journal 763 (2013), Januar, S. 51. http://dx.doi.org/10.1088/0004-637X/763/1/51. - DOI 10.1088/0004-637X/763/1/51

[Federrath u. a. 2010] Federrath, C. ; Roman-Duval, J. ; Klessen, R. S. ; SCHMidT, W. ; MAC LOW, M.-M.: Comparing the statistics of interstellar turbulence in simulations and observations. Solenoidal versus compressive turbulence forcing. In: Astronomy and Astrophysics 512 (2010), März, S. A81+. http://dx . doi.org/10.1051/0004-6361/200912437. - DOI 10.1051/0004-6361/200912437

[Ferland u. a. 1998] Ferland, G. J. ; Korista, K. T. ; Verner, D. A. ; Ferguson, J. W. ; Kingdon, J. B. ; Verner, E. M.: CLOUdY 90: Numerical Simulation of Plasmas and Their Spectra. In: The Publications of the Astronomical Society of the Pacific 110 (1998), Juli, S. 761-778. http://dx.doi.org/10.1086/316190. - DOI 10.1086/316190

[Frisch 1995] FRISCH, U.: Turbulence. The legacy of A.N. Kolmogorov. Cambridge: Cambridge University Press, 1995 http://adsabs.harvard.edu/ cgi-bin/nph-bib_query?bibcode=1995tlnk. book. . . . F\&db_key=AST

[Galtier u. Banerjee 2011] Galtien, S. ; BanerJee, S.: Exact Relation for Correlation Functions in Compressible Isothermal Turbulence. In: Physical Review Letters 107 (2011), September, Nr. 13, S. 134501. http://dx.doi.org/10.1103/ PhysRevLett.107.134501. - DOI 10.1103/PhysRevLett.107.134501

[Gao u. Solomon 2004] Gao, Y. ; Solomon, P. M.: The Star Formation Rate and Dense Molecular Gas in Galaxies. In: Astrophysical Journal 606 (2004), Mai, S. 271-290. http://dx.doi.org/10.1086/382999. - DOI 10.1086/382999

[Genzel u. a. 2010] Genzel, R. ; Tacconi, L. J. ; Gracia-Carpio, J. ; SternBerg, A. ; Cooper, M. C. ; Shapiro, K. ; Authors, 16 co: A study of the gas-star formation relation over cosmic time. In: Monthly Notices of the Royal Astronomical Society 407 (2010), Oktober, S. 2091-2108. http://dx.doi.org/ 10.1111/j.1365-2966.2010.16969.x. - DOI 10.1111/j.1365-2966.2010.16969.x

[Germano 1992] Germano, M.: Turbulence - The filtering approach. In: Journal of Fluid Mechanics 238 (1992), Mai, S. 325-336. http://dx.doi.org/10.1017/ S0022112092001733. - DOI 10.1017/S0022112092001733 
[Glover u. Jappsen 2007] Glover, S. C. O. ; JAppsen, A.-K.: Star Formation at Very Low Metallicity. I. Chemistry and Cooling at Low Densities. In: Astrophysical Journal 666 (2007), September, S. 1-19. http://dx.doi.org/10.1086/519445. - DOI $10.1086 / 519445$

[Gnedin u. a. 2009] Gnedin, N. Y. ; TAssis, K. ; Kravtsov, A. V.: Modeling Molecular Hydrogen and Star Formation in Cosmological Simulations. In: Astrophysical Journal (2009), S. 55-67. http://dx.doi.org/10.1088/0004-637X/697/1/55. - DOI 10.1088/0004-637X/697/1/55

[Hennebelle u. Chabrier 2008] Hennebelle, P. ; Chabrier, G.: Analytical Theory for the Initial Mass Function: CO Clumps and Prestellar Cores. In: Astrophysical Journal 684 (2008), September, S. 395-410. http://dx.doi.org/10.1086/ 589916. - DOI 10.1086/589916

[Hennebelle u. Chabrier 2009] Hennebelle, P. ; Chabrier, G.: Analytical Theory for the Initial Mass Function. II. Properties of the Flow. In: Astrophysical Journal 702 (2009), September, S. 1428-1442. http://dx.doi.org/10.1088/0004-637X/ 702/2/1428. - DOI 10.1088/0004-637X/702/2/1428

[Hennebelle u. Chabrier 2011] Hennebelle, P. ; Chabrier, G.: Analytical Star Formation Rate from Gravoturbulent Fragmentation. In: The Astrophysical Journal Letters 743 (2011), Dezember, S. L29. http://dx.doi.org/10.1088/ 2041-8205/743/2/L29. - DOI 10.1088/2041-8205/743/2/L29

[Hennebelle u. Chabrier 2013] Hennebelle, P. ; Chabrier, G.: Analytical Theory for the Initial Mass Function. III. Time Dependence and Star Formation Rate. In: Astrophysical Journal 770 (2013), Juni, S. 150. http://dx.doi.org/10.1088/ 0004-637X/770/2/150. - DOI 10.1088/0004-637X/770/2/150

[Hopkins u.a. 2013] Hopkins, P. F. ; Kereš, D. ; Murray, N.: Accretion does not drive the turbulence in galactic discs. In: Monthly Notices of the Royal Astronomical Society 432 (2013), Juli, S. 2639-2646. http://dx.doi.org/10.1093/ mnras/stt472. - DOI 10.1093/mnras/stt472

[Hughes u. a. 2013a] Hughes, A. ; Meidt, S. E. ; Colombo, D. ; Schinnerer, E. ; Pety, J. ; Leroy, A. K. ; Dobbs, C. L. ; García-Burillo, S. ; Thompson, T. A. ; Dumas, G. ; Schuster, K. F. ; Kramer, C.: A Comparative Study of Giant Molecular Clouds in M51, M33, and the Large Magellanic Cloud. In: Astrophysical Journal 779 (2013), Dezember, S. 46. http://dx.doi.org/10. 1088/0004-637X/779/1/46. - DOI 10.1088/0004-637X/779/1/46 
[Hughes u. a. 2013b] Hughes, A. ; Meidt, S. E. ; Schinnerer, E. ; Colombo, D. ; Pety, J. ; Leroy, A. K. ; Dobbs, C. L. ; García-Burillo, S. ; Thompson, T. A. ; Dumas, G. ; Schuster, K. F. ; Kramer, C.: Probability Distribution Functions of ${ }^{12} \mathrm{CO}(\mathrm{J}=1 \rightarrow 0)$ Brightness and Integrated Intensity in M51: The PAWS View. In: Astrophysical Journal 779 (2013), Dezember, S. 44. http://dx . doi .org/10.1088/0004-637X/779/1/44. - DOI 10.1088/0004-637X/779/1/44

[Hunter u. a. 2012] Hunter, D. A. ; Ficut-Vicas, D. ; Ashley, T. ; Brinks, E. ; Cigan, P. ; Elmegreen, B. G. ; Heesen, V. ; Herrmann, K. A. ; Johnson, M. ; Oh, S.-H. ; Rupen, M. P. ; Schruba, A. ; Simpson, C. E. ; Walter, F. ; Westpfahl, D. J. ; Young, L. M. ; Zhang, H.-X.: Little Things. In: The Astronomical Journal 144 (2012), November, S. 134. http://dx.doi.org/10. 1088/0004-6256/144/5/134. - DOI 10.1088/0004-6256/144/5/134

[Jeans 1902] Jeans, J. H.: The Stability of a Spherical Nebula. In: Royal Society of London Philosophical Transactions Series A 199 (1902), S. 1-53. http://dx. doi.org/10.1098/rsta.1902.0012. - DOI 10.1098/rsta.1902.0012

[Kennicutt u. Evans 2012] Kennicutt, R. C. ; Evans, N. J.: Star Formation in the Milky Way and Nearby Galaxies. In: Annual Review of Astronomy and Astrophysics 50 (2012), September, S. 531-608. http://dx.doi.org/10.1146/ annurev-astro-081811-125610. - DOI 10.1146/annurev-astro-081811-125610

[Kennicutt 1998] KennicutT, R. C. Jr.: The Global Schmidt Law in Star-forming Galaxies. In: Astrophysical Journal 498 (1998), Mai, S. 541. http://dx.doi. org/10.1086/305588. - DOI 10.1086/305588

[Kennicutt u. a. 2003] Kennicutt, R. C. Jr. ; Armus, L. ; Bendo, G. ; Calzetti, D. ; Dale, D. A. ; Draine, B. T. ; Engelbracht, C. W. ; Gordon, K. D. ; Grauer, A. D. ; Helou, G. ; Hollenbach, D. J. ; Jarrett, T. H. ; Kewley, L. J. ; Leitherer, C. ; Li, A. ; Malhotra, S. ; Regan, M. W. ; Rieke, G. H. ; Rieke, M. J. ; Roussel, H. ; Smith, J.-D. T. ; Thornley, M. D. ; Walter, F.: SINGS: The SIRTF Nearby Galaxies Survey. In: The Publications of the Astronomical Society of the Pacific 115 (2003), August, S. 928-952. http: //dx.doi.org/10.1086/376941. - DOI 10.1086/376941

[Kippenhahn u.a. 2012] Kippenhahn, R. ; Weigert, A. ; Weiss, A.: Stellar Structure and Evolution. Springer-Verlag Berlin Heidelberg, 2012. http: //dx.doi.org/10.1007/978-3-642-30304-3. http://dx.doi.org/10.1007/ 978-3-642-30304-3

[Kolmogorov 1941a] Kolmogorov, A. N.: Dissipation of energy in locally isotropic turbulence. In: Dokl. Acad. Nauk. SSSR 32 (1941), S. 16-18 
[Kolmogorov 1941b] Kolmogorov, A. N.: On degeneration (decay) of isotropic turbulence in an incompressible viscous liquid. In: Dokl. Acad. Nauk. SSSR 31 (1941), S. 538-540

[Kolmogorov 1941c] Kolmogorov, A. N.: The local structure of turbulence in incompressible viscous fluid for very large Reynolds number. In: Dokl. Acad. Nauk. SSSR 30 (1941), S. 9-13

[Kotulla u.a. 2009] Kotulla, R. ; Fritze, U. ; Weilbacher, P. ; Anders, P.: GALEV evolutionary synthesis models - I. Code, input physics and web interface. In: Monthly Notices of the Royal Astronomical Society 396 (2009), Juni, S. 462-484. http://dx.doi.org/10.1111/j.1365-2966.2009.14717.x. - DOI 10.1111/j.1365-2966.2009.14717.x

[Kraljic u. a. 2012] Kraljic, K. ; Bournaud, F. ; Martig, M.: The Two-phase Formation History of Spiral Galaxies Traced by the Cosmic Evolution of the Bar Fraction. In: Astrophysical Journal 757 (2012), September, S. 60. http://dx. doi.org/10.1088/0004-637X/757/1/60. - DOI 10.1088/0004-637X/757/1/60

[Kritsuk u. a. 2007] Kritsuk, A. G. ; Norman, M. L. ; Padoan, P. ; Wagner, R.: The Statistics of Supersonic Isothermal Turbulence. In: Astrophysical Journal 665 (2007), August, S. 416-431. http://dx.doi.org/10.1086/519443. - DOI $10.1086 / 519443$

[Krumholz 2013] Krumholz, M. R.: Star Formation and the Atomic-Molecular Transition. In: Wong, T. (Hrsg.) ; OtT, J. (Hrsg.): IAU Symposium Bd. 292, 2013 (IAU Symposium), S. 227-234

[Krumholz 2014] Krumholz, M. R.: The Big Problems in Star Formation: the Star Formation Rate, Stellar Clustering, and the Initial Mass Function. In: ArXiv e-prints (2014), Februar

[Krumholz u. McKee 2005] Krumholz, M. R. ; McKee, C. F.: A General Theory of Turbulence-regulated Star Formation, from Spirals to Ultraluminous Infrared Galaxies. In: Astrophysical Journal 630 (2005), September, S. 250-268. http: //dx.doi.org/10.1086/431734. - DOI 10.1086/431734

[Lada u. a. 2010] LadA, C. J. ; Lombardi, M. ; Alves, J. F.: On the Star Formation Rates in Molecular Clouds. In: Astrophysical Journal 724 (2010), November, S. 687-693. http://dx.doi.org/10.1088/0004-637X/724/1/687. - DOI $10.1088 / 0004-637 \mathrm{X} / 724 / 1 / 687$

[Larson 1981] LARSON, R. B.: Turbulence and star formation in molecular clouds. In: Monthly Notices of the Royal Astronomical Society 194 (1981), März, S. 809-826 
[Leroy u.a. 2008] Leroy, A. K. ; Walter, F. ; Brinks, E. ; Bigiel, F. ; De Blok, W. J. G. ; Madore, B. ; Thornley, M. D.: The Star Formation Efficiency in Nearby Galaxies: Measuring Where Gas Forms Stars Effectively. In: The Astronomical Journal 136 (2008), Dezember, S. 2782-2845. http://dx.doi.org/10.1088/0004-6256/136/6/2782 - - DOI 10.1088/0004$6256 / 136 / 6 / 2782$

[McKee u. Krumholz 2010] McKee, C. F. ; Krumholz, M. R.: The Atomic-toMolecular Transition in Galaxies. III. A New Method for Determining the Molecular Content of Primordial and Dusty Clouds. In: Astrophysical Journal 709 (2010), Januar, S. 308-320. http://dx.doi.org/10.1088/0004-637X/709/1/308. DOI $10.1088 / 0004-637 \mathrm{X} / 709 / 1 / 308$

[McKee u. Ostriker 2007] McKee, C. F. ; Ostriker, E. C.: Theory of Star Formation. In: Annual Review of Astronomy and Astrophysics 45 (2007), September, S. 565-687. http://dx.doi.org/10.1146/annurev.astro.45.051806.110602. - DOI 10.1146/annurev.astro.45.051806.110602

[McKee u. Ostriker 1977] McKee, C. F. ; Ostriker, J. P.: A theory of the interstellar medium - Three components regulated by supernova explosions in an inhomogeneous substrate. In: Astrophysical Journal 218 (1977), November, S. 148-169. http://dx.doi.org/10.1086/155667. - DOI 10.1086/155667

[Miura u. a. 2012] Miura, R. E. ; Kohno, K. ; Tosaki, T. ; EspadA, D. ; Hwang, N. ; Kuno, N. ; COAuthors, 10: Giant Molecular Cloud Evolutions in the Nearby Spiral Galaxy M33. In: Astrophysical Journal 761 (2012), Dezember, S. 37. http://dx.doi.org/10.1088/0004-637X/761/1/37. - DOI 10.1088/0004-637X/761/1/37

[Molinari u.a. 2014] Molinari, S. ; Bally, J. ; Glover, S. ; Moore, T. ; Noriega-Crespo, A. ; Plume, R. ; Testi, L. ; VÁzquez-Semadeni, E. ; ZaVagno, A. ; Bernard, J.-P. ; Martin, P.: The Milky Way as a Star Formation Engine. In: ArXiv e-prints (2014), Februar

[Monaco u.a. 2012] Monaco, P. ; Murante, G. ; Borgani, S. ; Dolag, K.: Schmidt-Kennicutt relations in SPH simulations of disc galaxies with effective thermal feedback from supernovae. In: Monthly Notices of the Royal Astronomical Society 421 (2012), April, S. 2485-2497. http://dx.doi.org/10.1111/j. 1365-2966.2012.20482.x. - DOI 10.1111/j.1365-2966.2012.20482.x

[Munshi u.a. 2013] Munshi, F. ; Governato, F. ; Brooks, A. M. ; Christensen, C. ; Shen, S. ; Loebman, S. ; Moster, B. ; Quinn, T. ; WadsLEY, J.: Reproducing the Stellar Mass/Halo Mass Relation in Simulated $\Lambda$ CDM 
Galaxies: Theory versus Observational Estimates. In: Astrophysical Journal 766 (2013), März, S. 56. http://dx.doi.org/10.1088/0004-637X/766/1/56. - DOI $10.1088 / 0004-637 \mathrm{X} / 766 / 1 / 56$

[Murante u.a. 2010] Murante, G. ; Monaco, P. ; Giovalli, M. ; Borgani, S. ; Diaferio, A.: A subresolution multiphase interstellar medium model of star formation and supernova energy feedback. In: Monthly Notices of the Royal Astronomical Society 405 (2010), Juli, S. 1491-1512. http://dx.doi.org/10. 1111/j.1365-2966.2010.16567.x. - DOI 10.1111/j.1365-2966.2010.16567.x

[Murray 2011] Murray, N.: Star Formation Efficiencies and Lifetimes of Giant Molecular Clouds in the Milky Way. In: Astrophysical Journal 729 (2011), März, S. 133. http://dx.doi.org/10.1088/0004-637X/729/2/133. - DOI 10.1088/0004-637X/729/2/133

[Navarro u. a. 1997] Navarro, J. F. ; Frenk, C. S. ; White, S. D. M.: A Universal Density Profile from Hierarchical Clustering. In: Astrophysical Journal 490 (1997), Dezember, S. 493. http://dx.doi.org/10.1086/304888. - DOI 10.1086/304888

[Onodera u. a. 2010] Onodera, S. ; Kuno, N. ; Tosaki, T. ; Kohno, K. ; NAKAnishi, K. ; Sawada, T. ; Muraoka, K. ; Komugi, S. ; Miura, R. ; Kaneko, H. ; Hirota, A. ; Kawabe, R.: Breakdown of Kennicutt-Schmidt Law at Giant Molecular Cloud Scales in M33. In: The Astrophysical Journal Letters 722 (2010), Oktober, S. L127-L131. http://dx.doi .org/10.1088/2041-8205/722/2/L127. - DOI 10.1088/2041-8205/722/2/L127

[O'Shea u. a. 2004] O'Shea, B. W. ; Bryan, G. ; Bordner, J. ; Norman, M. L. ; Abel, T. ; Harkness, R. ; Kritsuk, A.: Introducing Enzo, an AMR Cosmology Application. In: ArXiv Astrophysics e-prints (2004), März

[Padoan u. a. 2013] Padoan, P. ; Federrath, C. ; Chabrier, G. ; Evans, N. J. II ; Johnstone, D. ; Jørgensen, J. K. ; McKee, C. F. ; Nordlund, Å.: The Star Formation Rate of Molecular Clouds. In: ArXiv e-prints (2013), Dezember

[Padoan u. a. 2012] Padoan, P. ; Haugbølle, T. ; Nordlund, A.: A Simple Law of Star Formation. In: The Astrophysical Journal Letters 759 (2012), November, S. L27. http://dx.doi.org/10.1088/2041-8205/759/2/L27. - DOI $10.1088 / 2041-8205 / 759 / 2 / \mathrm{L} 27$

[Padoan u. Nordlund 2011] Padoan, P. ; Nordlund, Å.: The Star Formation Rate of Supersonic Magnetohydrodynamic Turbulence. In: Astrophysical Journal 730 (2011), März, S. 40. http://dx.doi .org/10.1088/0004-637X/730/1/40. - DOI $10.1088 / 0004-637 \mathrm{X} / 730 / 1 / 40$ 
[Planck Collaboration u.a. 2013] Planck Collaboration ; Ade, P. A. R. ; Aghanim, N. ; Alves, M. I. R. ; Armitage-Caplan, C. ; Arnaud, M. ; Ashdown, M. ; Atrio-Barandela, F. ; Aumont, J. ; Aussel, H. ; Al. et: Planck 2013 results. I. Overview of products and scientific results. In: ArXiv e-prints (2013), März

[Pope 2000] Pope, S. B.: Turbulent Flows. Cambridge University Press, 2000

[Raiteri u. a. 1996] Raiteri, C. M. ; Villata, M. ; Navarro, J. F.: Simulations of Galactic chemical evolution. I. O and Fe abundances in a simple collapse model. In: Astronomy and Astrophysics 315 (1996), November, S. 105-115

[Renaud u. a. 2013] Renaud, F. ; Bournaud, F. ; Emsellem, E. ; Elmegreen, B. ; Teyssier, R. ; Alves, J. ; Chapon, D. ; Combes, F. ; Dekel, A. ; Gabor, J. ; Hennebelle, P. ; Kraljic, K.: A sub-parsec resolution simulation of the Milky Way: global structure of the interstellar medium and properties of molecular clouds. In: Monthly Notices of the Royal Astronomical Society (2013), Oktober. http://dx.doi.org/10.1093/mnras/stt1698. - DOI 10.1093/mnras/stt1698

[Salpeter 1955] SAlpeter, E. E.: The Luminosity Function and Stellar Evolution. In: Astrophysical Journal 121 (1955), Januar, S. 161-+. http://dx.doi.org/ 10.1086/145971. - DOI 10.1086/145971

[Schade u. a. 2014] Schade, H. ; Kunz, E. ; Kameier, F.. ; Paschereit, C. O.: Strömungslehre (de Gruyter Studium). de Gruyter, 2014

[Schinnerer u. a. 2013] Schinnerer, E. ; Meidt, S. E. ; Pety, J. ; Hughes, A. ; Colombo, D. ; García-Burillo, S. ; Schuster, K. F. ; Dumas, G. ; Dobbs, C. L. ; Leroy, A. K. ; Kramer, C. ; Thompson, T. A. ; Regan, M. W.: The PdBI Arcsecond Whirlpool Survey (PAWS). I. A Cloud-scale/Multi-wavelength View of the Interstellar Medium in a Grand-design Spiral Galaxy. In: Astrophysical Journal 779 (2013), Dezember, S. 42. http://dx.doi.org/10.1088/0004-637X/ 779/1/42. - DOI 10.1088/0004-637X/779/1/42

[Schmidt 1959] Schmidt, M.: The Rate of Star Formation. In: Astrophysical Journal 129 (1959), März, S. 243. http://dx.doi.org/10.1086/146614. - DOI $10.1086 / 146614$

[Schmidt 2014] Schmidt, W.: Large Eddy Simulations in Astrophysics. In: ArXiv e-prints (2014), April

[Schmidt u. a. 2014] Schmidt, W. ; Almgren, A. S. ; Braun, H. ; Engels, J. F. ; Niemeyer, J. C. ; Schulz, J. ; Mekuria, R. R. ; Aspden, A. J. ; Bell, J. B.: 
Cosmological fluid mechanics with adaptively refined large eddy simulations. In: Monthly Notices of the Royal Astronomical Society 440 (2014), Juni, S. 3051-3077. http://dx.doi.org/10.1093/mnras/stu501. - DOI 10.1093/mnras/stu501

[Schmidt u. Federrath 2011] Schmidt, W. ; Federrath, C.: A fluid-dynamical subgrid scale model for highly compressible astrophysical turbulence. In: Astronomy and Astrophysics 528 (2011), April, S. A106+. http://dx.doi.org/10. 1051/0004-6361/201015630. - DOI 10.1051/0004-6361/201015630

[Schmidt u. a. 2006] Schmidt, W. ; Niemeyer, J. C. ; Hillebrandt, W.: A localised subgrid scale model for fluid dynamical simulations in astrophysics. I. Theory and numerical tests. In: Astronomy and Astrophysics 450 (2006), April, S. 265281. http://dx.doi .org/10.1051/0004-6361:20053617. - DOI 10.1051/00046361:20053617

[Schneider u. a. 2014] Schneider, N. ; Ossenkopf, V. ; Csengeri, T. ; Klessen, R. ; Federrath, C. ; Tremblin, P. ; Girichidis, P. ; Bontemps, S. ; Andre, P.: Understanding star formation in molecular clouds I. A universal probability distribution of column densities ? In: ArXiv e-prints (2014), März

[Schruba 2013] SchrubA, A.: The Star Formation Relation in Nearby Galaxies. In: Wong, T. (Hrsg.) ; Oтt, J. (Hrsg.): IAU Symposium Bd. 292, 2013 (IAU Symposium), S. 311-318

[Shetty u. a. 2012] Shetty, R. ; Beaumont, C. N. ; Burton, M. G. ; Kelly, B. C. ; Klessen, R. S.: The linewidth-size relationship in the dense interstellar medium of the Central Molecular Zone. In: Monthly Notices of the Royal Astronomical Society 425 (2012), September, S. 720-729. http://dx.doi.org/10.1111/j. 1365-2966.2012.21588.x. - DOI 10.1111/j.1365-2966.2012.21588.x

[Springel 2010] SPRINGEL, V.: E pur si muove: Galilean-invariant cosmological hydrodynamical simulations on a moving mesh. In: Monthly Notices of the Royal Astronomical Society 401 (2010), Januar, S. 791-851. http://dx.doi.org/10. 1111/j.1365-2966.2009.15715.x. - DOI 10.1111/j.1365-2966.2009.15715.x

[Springel u. Hernquist 2003] Springel, V. ; Hernquist, L.: Cosmological smoothed particle hydrodynamics simulations: a hybrid multiphase model for star formation. In: Monthly Notices of the Royal Astronomical Society 339 (2003), Februar, S. 289-311. http://dx.doi.org/10.1046/j.1365-8711.2003.06206.x. - DOI $10.1046 /$ j.1365-8711.2003.06206.x

[Springel u. a. 2001] Springel, V. ; Yoshida, N. ; White, S. D. M.: GADGET: a code for collisionless and gasdynamical cosmological simulations. In: New Astro- 
nomy 6 (2001), April, S. 79-117. http://dx.doi.org/10.1016/S1384-1076(01) 00042-2. - DOI 10.1016/S1384-1076(01)00042-2

[Stilp u. a. 2013] Stilp, A. M. ; Dalcanton, J. J. ; Warren, S. R. ; Weisz, D. R. ; Skillman, E. ; Ott, J. ; Williams, B. F. ; Dolphin, A. E.: Timescales on which Star Formation Affects the Neutral Interstellar Medium. In: Astrophysical Journal 772 (2013), August, S. 124. http://dx.doi.org/10.1088/0004-637X/ 772/2/124. - DOI 10.1088/0004-637X/772/2/124

[Stinson u. a. 2006] Stinson, G. ; Seth, A. ; Katz, N. ; Wadsley, J. ; GoVERnato, F. ; Quinn, T.: Star formation and feedback in smoothed particle hydrodynamic simulations - I. Isolated galaxies. In: Monthly Notices of the Royal Astronomical Society 373 (2006), Dezember, S. 1074-1090. http: //dx.doi.org/10.1111/j.1365-2966.2006.11097.x. - DOI 10.1111/j.13652966.2006.11097.x

[Stinson u.a. 2010] Stinson, G. S. ; Bailin, J. ; Couchman, H. ; Wadsley, J. ; Shen, S. ; Nickerson, S. ; Brook, C. ; Quinn, T.: Cosmological galaxy formation simulations using smoothed particle hydrodynamics. In: Monthly Notices of the Royal Astronomical Society 408 (2010), Oktober, S. 812-826. http: //dx.doi.org/10.1111/j.1365-2966.2010.17187.x. - DOI 10.1111/j.13652966.2010.17187.x

[Stinson u. a. 2013] Stinson, G. S. ; Brook, C. ; Macciò, A. V. ; Wadsley, J. ; Quinn, T. R. ; Couchman, H. M. P.: Making Galaxies In a Cosmological Context: the need for early stellar feedback. In: Monthly Notices of the Royal Astronomical Society 428 (2013), Januar, S. 129-140. http://dx.doi.org/10. 1093/mnras/sts028. - DOI 10.1093/mnras/sts028

[Tan u. a. 2013] TAn, J. C. ; Shaske, S. N. ; VAn Loo, S.: Molecular Clouds: Internal Properties, Turbulence, Star Formation and Feedback. In: Wong, T. (Hrsg.) ; Oтт, J. (Hrsg.): IAU Symposium Bd. 292, 2013 (IAU Symposium), S. $19-28$

[Teyssier 2002] TEYssiER, R.: Cosmological hydrodynamics with adaptive mesh refinement. A new high resolution code called RAMSES. In: Astronomy and Astrophysics 385 (2002), April, S. 337-364. http://dx .doi .org/10.1051/0004-6361: 20011817. - DOI 10.1051/0004-6361:20011817

[Thornton u. a. 1998] Thornton, K. ; Gaudlitz, M. ; JankA, H.-T. ; Steinmetz, M.: Energy Input and Mass Redistribution by Supernovae in the Interstellar Medium. In: Astrophysical Journal 500 (1998), Juni, S. 95-+. http://dx.doi. org/10.1086/305704. - DOI 10.1086/305704 
[Toomre 1964] ToOmRe, A.: On the gravitational stability of a disk of stars. In: Astrophysical Journal 139 (1964), Mai, S. 1217-1238. http://dx.doi.org/10. 1086/147861. - DOI 10.1086/147861

[Vázquez-Semadeni u. a. 2003] VÁzquez-Semadeni, E. ; Ballesteros-Paredes, J. ; Klessen, R. S.: A Holistic Scenario of Turbulent Molecular Cloud Evolution and Control of the Star Formation Efficiency: First Tests. In: The Astrophysical Journal Letters 585 (2003), März, S. L131-L134. http://dx.doi.org/10.1086/ 374325. - DOI 10.1086/374325

[Walter u.a. 2008] Walter, F. ; Brinks, E. ; De Blok, W. J. G. ; Bigiel, F. ; Kennicutt, R. C. Jr. ; Thornley, M. D. ; Leroy, A.: ThingS: The H I Nearby Galaxy Survey. In: The Astronomical Journal 136 (2008), Dezember, S. 2563-2647. http://dx.doi.org/10.1088/0004-6256/136/6/2563. - DOI $10.1088 / 0004-6256 / 136 / 6 / 2563$

[Wang u. a. 2010] Wang, H.-H. ; Klessen, R. S. ; Dullemond, C. P. ; van Den Bosch, F. C. ; Fuchs, B.: Equilibrium initialization and stability of threedimensional gas discs. In: Monthly Notices of the Royal Astronomical Society 407 (2010), September, S. 705-720. http://dx . doi .org/10.1111/j .1365-2966. 2010.16942.x. - DOI 10.1111/j.1365-2966.2010.16942.x

[Weinberg 2008] WeinberG, S.: Cosmology. Oxford University Press, 2008

[Wilson 2013] Wilson, C. D.: Molecular gas and dust in spiral galaxies. In: Wong, T. (Hrsg.) ; Отт, J. (Hrsg.): IAU Symposium Bd. 292, 2013 (IAU Symposium), S. $119-126$

[Wise u.a. 2012] Wise, J. H. ; Abel, T. ; Turk, M. J. ; Norman, M. L. ; Smith, B. D.: The birth of a galaxy - II. The role of radiation pressure. In: Monthly Notices of the Royal Astronomical Society 427 (2012), November, S. 311-326. http://dx.doi.org/10.1111/j.1365-2966.2012.21809.x. - DOI 10.1111/j.1365-2966.2012.21809.x 


\section{Danksagungen}

Mein erster Dank gebührt meinen Betreuern Jens Niemeyer und Wolfram Schmidt, die es mir überhaupt erst ermöglichten, an diesem vielfältigen und interessanten Projekt zu arbeiten und mir die Freiheit gaben, es nach meinen Vorstellungen mit Leben zu füllen. Ohne Eure engagierte Betreuung sowie die vielen hilfreichen Anregungen und Diskussionen wäre dies hier nicht möglich gewesen.

Ich möchte meinem Korreferenten Dominik Schleicher für seine bereitwillige, kompetente Unterstützung zu danken.

Dankbar bin ich auch für das freundschaftlich-kollegiale Umfeld am Institut für Astrophysik in Göttingen, insbesondere innerhalb der Arbeitsgruppe für Kosmologie. Hervorheben möchte ich hierbei die Mitinsassen meines Büros E04-115:

Christoph und Jeff. Vielen Dank, dass ich Teil dieser speziellen Rasselbande sein durfte, innerhalb der man sich nicht nur zu fachlichen oder technischen Themen austauschen konnte, sondern auch weit darüber hinaus. Es war mir eine große Freude, mit Euch zu arbeiten.

Ein gewaltiger Dank geht an meine Freundin Nina, die ich sehr liebe. Danke, dass Du mich in dieser Zeit ertragen und motiviert hast.

Nicht zuletzt möchte ich meinen Eltern und meiner Familie für den Beistand in allen Belangen - also auch in meinem Promotionsvorhaben - danken. Ohne Euch wäre ich wohl kaum der, der ich bin, dort wo ich bin.

An dieser Stelle möchte ich nun allen bisher unerwähnten Freunden und Kollegen danken, die mich auf meinem Weg begleitet, oder auch bei diesem Unterfangen in irgendeiner Form unterstützt haben.

Out of all the things I have lost, I miss my mind the most.

- Mark Twain 


\section{Anhang A \\ Publikationen}

A.1 Publikation Braun \& Schmidt, 2012 
Monthly Notices

\title{
A semi-analytic model of the turbulent multi-phase interstellar medium
}

\author{
H. Braun ${ }^{\star}$ and W. Schmidt \\ Institut für Astrophysik, Universität Göttingen, Friedrich-Hund Platz 1, D-37077 Göttingen, Germany
}

Accepted 2011 September 26. Received 2011 August 12; in original form 2011 April 29

\begin{abstract}
We present a semi-analytic model for the interstellar medium that considers local processes and structures of turbulent star-forming gas. A volume element of the interstellar medium is described as a multi-phase system, comprising a cold and a warm gas phase in effective (thermal plus turbulent) pressure equilibrium and a stellar component. The cooling instability of the warm gas feeds the cold phase, while various heating processes transfer cold gas to the warm phase. The cold phase consists of clumps embedded in diffuse warm gas, where only the molecular fraction of the cold gas may be converted into stars. The fraction of molecular gas is approximately calculated, using a Strömgren-like approach and the efficiency of star formation is determined by the state of the cold gas and the turbulent velocity dispersion on the clump length-scale. Gas can be heated by supernovae and ultraviolet emission of massive stars, according to the evolutionary stages of the stellar populations and the initial mass function. Since turbulence has a critical impact on the shape of the gaseous phases, on the production of molecular hydrogen and on the formation of stars, the consistent treatment of turbulent energy - the kinetic energy of unresolved motions - is an important new feature of our model. Besides turbulence production by supernovae and the cooling instability, we also take into account the forcing by large-scale motions.

We formulate a set of ordinary differential equations, which statistically describes star formation and the exchange between the different budgets of mass and energy in a region of the interstellar medium with given mean density, size, metallicity and external turbulence forcing. By exploring the behaviour of the solutions, we find equilibrium states, in which the star formation efficiencies are consistent with observations. Kennicutt-Schmidt-like relations naturally arise from the equilibrium solutions, while conventional star formation models in numerical simulations impose such relations with observed efficiency parameters as phenomenological calibrations.

Beyond the semi-analytic approach, a potential application is a complete sub-grid scale model of the unresolved multi-phase structure, star formation and turbulence in simulations of galaxies or in cosmological simulations. The formulation presented in this article combines various models focusing on particular processes and yet can be adopted to specific applications, depending on the range of resolved length-scales.
\end{abstract}

Key words: turbulence - methods: numerical - stars: formation - ISM: structure - galaxies: ISM.

\section{INTRODUCTION}

The capabilities of contemporary supercomputing enable us to model the evolution of the baryonic gas in the universe with unprecedented sophistication. Adaptive methods such as smoothed particle hydrodynamics (SPH) and adaptive mesh refinement (AMR) in Eulerian grid codes allow us to cover a huge dynamic range such

^E-mail: hbraun@astro.physik.uni-goettingen.de that simulations of the formation and evolution of galaxies from cosmological initial conditions at high resolution $(\sim 100 \mathrm{pc})$ are within reach (Gnedin \& Kravtsov 2010; Agertz, Teyssier \& Moore 2011). In simulations of isolated disc galaxies, it is feasible to resolve length-scales down to $\sim 10$ pc (Agertz et al. 2009; Tasker \& Tan 2009). Computations on these length-scales entail the problem to account for various physical processes in the multi-phase interstellar medium (ISM; Mayer, Governato \& Kaufmann 2008). Notwithstanding the high numerical resolution that can be achieved, several important processes cannot be fully resolved and have to be described by means of a sub-grid scale (SGS) model. 
The distribution of the gas among the different phases of the ISM is controlled by the following physical processes. The fragmentation of warm neutral gas (number density $n \lesssim 1 \mathrm{~cm}^{-3}$ and temperature $T \gtrsim 10^{4} \mathrm{~K}$ ) is driven by gravitational instabilities on length-scales $\sim 0.1, \ldots, 1 \mathrm{kpc}$ (e.g. Toomre 1964; Wada, Meurer \& Norman 2002; Kravtsov 2003; Li, Mac Low \& Klessen 2005; Wada \& Norman 2007; Agertz et al. 2009). The gravitational contraction of gas is supported by cooling processes in converging flows that produce the cold neutral phase $\left(n \gtrsim 10 \mathrm{~cm}^{-3}, T \lesssim 10^{3} \mathrm{~K}\right)$. Gravitational and cooling instabilities, and, possibly, magnetic fields act in concert to form dense star-forming clouds, in which molecular hydrogen is produced at densities $\gtrsim 100 \mathrm{~cm}^{-3}$ (e.g. Dobbs et al. 2008; Robertson \& Kravtsov 2008; Tasker \& Tan 2009). The radiation from hot massive stars and blast waves from supernovae $(\mathrm{SNe})$ feeds energy back into the ISM. Gravity, cooling, and stellar feedback are potential drivers of turbulence (de Avillez \& Breitschwerdt 2004; Elmegreen \& Scalo 2004; Mac Low \& Klessen 2004; Bournaud et al. 2010; Burkert et al. 2010; Klessen \& Hennebelle 2010; Federrath et al. 2011), which, in turn, has an impact on the stability of the gas (Bonazzola et al. 1992; Romeo, Burkert \& Agertz 2010).

In large-scale simulations, where the smallest resolved lengthscales range from the scale of star-forming regions to galactic scales, it is a major challenge to account for the sub-resolution structure and dynamics of the ISM (recent reviews are given by McKee \& Ostriker 2007 and Hensler 2009). On the one hand, isolated disc galaxy simulations serve as idealized models of galaxy evolution that avoid some of the difficulties one faces in cosmological simulations (Dobbs et al. 2008; Robertson \& Kravtsov 2008; Agertz et al. 2009; Tasker \& Tan 2009; Bournaud et al. 2010; Dobbs \& Pringle 2010). Although the highly artificial initial conditions are problematic, isolated discs can be used to study dynamical properties of the ISM at high resolutions and to test advanced models of unresolved processes. Because artefacts may result from discs that are adiabatically unstable, Wang et al. (2010) defined an adiabatic disc that is stable over the rotation time-scale. On the other hand, substantial efforts have been made to zoom into haloes from cosmological simulations and to re-simulate galaxies evolving from those haloes at the highest feasible resolution (Governato et al. 2007; Gnedin \& Kravtsov 2010; Governato et al. 2010; Greif et al. 2010; Agertz et al. 2011)

Several models were developed in the past to describe the multiphase ISM (e.g. McKee \& Ostriker 1977; Yepes et al. 1997; Gnedin 1998; Klypin 1998; Hultman \& Pharasyn 1999; Stinson et al. 2006). An often-used type of model for star formation and stellar feedback in cosmological SPH simulations is described in Springel \& Hernquist (2003) (SH03), which is an adaptation of the model introduced by Yepes et al. (1997). Basically, rate equations for the densities of the cold and hot gas phases are formulated, including sources and sinks related to star formation and feedback from supernovae. Recently, a variety of phenomenological models that treat particular physical processes in the ISM have been proposed (e.g. Gnedin, Tassis \& Kravtsov 2009; Joung, Mac Low \& Bryan 2009; Krumholz, McKee \& Tumlinson 2009; Murante et al. 2010; Ostriker, McKee \& Leroy 2010; Padoan \& Nordlund 2011). Some of these models are designed to account for SGS physics in numerical simulations. Others are mainly intended to obtain analytical or semi-analytical predictions that can be compared to observations. Even so, particular components of the latter class of models could be incorporated into an SGS model. In the following, we briefly review these models from the perspective of the physical processes involved.
Padoan \& Nordlund (2011, hereafter PN11) parametrize the star formation rate per free-fall time as a function of the virial parameter, i.e. the turbulent velocity dispersion relative to the specific gravitational energy, by using data from forced isothermal magnetohydrodynamic (MHD) turbulence simulations. Following Krumholz \& McKee (2005, hereafter KM05), the star formation rate is calculated by integrating density fluctuations beyond a critical density that is given by the virial parameter and the Mach number of the turbulent cold neutral medium. However, as pointed out by Krumholz et al. (2009, hereafter KMT09), new observations reveal a tight correlation between the molecular hydrogen surface density and the star formation rate. They present an analytic model that includes approximate calculations of molecular hydrogen fraction from a spherical-cloud model and the star formation efficiency per freefall time on the basis of the numerical parametrization in KM05. This model reproduces the Kennicutt-Schmidt relation between the star formation rate and the surface density on length-scales of the order of a kiloparsec in recent surveys.

By assuming a constant star formation efficiency, the formation of molecular hydrogen in cosmological simulations is modelled by an approximate treatment of shielding and photodissociation in Gnedin et al. (2009, hereafter GTK09). As in KMT09, the star formation rate is assumed to be proportional to the molecular hydrogen density rather than the density of the cold neutral medium. The unresolved density structure of the gas is parametrized by a clumping factor, and the efficiency of star formation per free-fall time in molecular clouds is set to 1 per cent. Using this model, Gnedin \& Kravtsov (2010) investigate the Kennicutt-Schmidt relation in galaxies at high redshifts. For simulations of isolated discs with molecular hydrogen chemistry, see Dobbs et al. (2008) and Robertson \& Kravtsov (2008).

The KMT09 and GTK09 models focus on molecular hydrogen to predict the star formation rate, whereas the multi-phase structure and the turbulent dynamics of the ISM are not addressed explicitly. In contrast, Koppen, Theis \& Hensler (1998) formulate a dynamical model for the evolution of a massive and a low-mass star component and clouds embedded in hot gas, with various interaction processes. In a similar way, the model of Springel \& Hernquist (2003) considers interacting cold and warm phases and stars. A simple multi-phase SGS model of star formation and supernova feedback is proposed by Murante et al. (2010). By assuming that the amount of molecular hydrogen is controlled by the pressure of the ISM, rate equations for the mass and the energy of a cold and a warm phase are solved in addition to the mass that is converted into stars. Ostriker et al. (2010) present a considerably more detailed analytical model that separates the ISM into a diffusive gas component and into gravitationally bound clouds, in which stars are formed at a rate that is proportional to their mass. The basic parameters of this model are the ratio of the thermal to the effective pressure (i.e. the sum of thermal, turbulent and magnetic pressures) of the diffusive gas, the fraction of warm diffusive gas (complementing a cold diffusive phase) and the star formation efficiency of the clouds. The main idea is that the radiation of young massive stars heats the diffusive ISM and the mass exchange between the diffusive components and the clouds regulates star formation. Turbulence and the conversion of atomic into molecular hydrogen are not decisive for the regulation process.

To include SN feedback in cosmological simulations, for example, Stinson et al. (2006) model the impact of SN blast waves on the thermal structure of the ISM. In contrast, Joung et al. (2009, hereafter JMB09) propose a non-thermal treatment of the SN feedback. They formulate a dynamical equation to compute the numerically unresolved turbulent pressure of the ISM, with the rate of energy 
1840

\section{H. Braun and W. Schmidt}

injection by SN blast waves as source term (internal turbulence driving). The turbulent pressure is proportional to the energy density of numerically unresolved turbulent velocity fluctuations. The coefficients of the equation for the turbulent pressure are calibrated on galactic-scale simulations of the ISM. A similar approach is utilized in Scannapieco \& Brüggen (2010, hereafter SB10) for turbulence in galaxy outflows.

Although feedback models using the turbulent pressure are promising, JMB09 and SB10 do not account for the increase of the turbulent pressure by the energy transfer from resolved to unresolved scales via the turbulent cascade (external turbulence driving). We expect this production channel to be important because turbulence in the ISM is to some extent driven by gravitational instabilities on large, galactic scales (Klessen \& Hennebelle 2010; Romeo et al. 2010). For the local computation in numerical simulations, Schmidt \& Federrath (2011, hereafter SF11) formulated and tested an SGS model for highly compressible turbulence. This model is also based on a dynamical equation for the numerically unresolved turbulent energy. However, in addition to diffusion and dissipation terms, SGS turbulent energy is produced by the shear of resolved small-scale fluctuations, i.e. the turbulent cascade. The rate of production by the turbulent cascade is called the turbulent energy flux. Simulations of forced supersonic turbulence (Schmidt et al. 2009; Federrath et al. 2010) were used to verify a new closure for the compressible turbulent energy flux. In large eddy simulations (LES), a closure is an approximation to a quantity that depends on unresolved density and velocity fluctuations. Moreover, it is demonstrated that the SGS model fulfils several basic requirements, such as a constant mean dissipation rate, independent of the numerical resolution and a power-law scaling of the SGS turbulent energy. For compressible turbulence driven by large-scale instabilities, this SGS model is the only model for computing the turbulent pressure consistently that has been systematically tested so far. Feedback can be included as an additional production term in the SGS turbulent energy equation. Since the unresolved turbulent velocity fluctuations in galaxy simulations are comparable to the speed of sound, we expect significant effects of the corresponding turbulent pressure, particularly with regard to the regulation of star formation.

The aim of this work is to bring together different approaches, using the SH03 model as a basic framework. Our treatment of star formation and molecular hydrogen formation is guided by KM05, KMT09 and PN11. To heat the interstellar gas, Lyman-continuum (Lyc) radiation of young massive stars and supernova feedback are calculated from the modelled star formation history, assuming the Chabrier (2001) initial mass function (IMF). We incorporate internal turbulence driving by the thermal instability and by a nonthermal fraction of supernovae feedback, as in JMB09. By adding the turbulent pressure to the thermal pressure, turbulence influences the pressure balance between the phases and, in the highly turbulent regime, it significantly affects the gravitationally unstable mass fraction in the cold-gas phase. The key to the fluid-dynamical computation of the turbulent energy, including external driving via a turbulent cascade, is SF11.

In this paper, we devise a semi-analytic formulation to describe the evolution of the two gas phases, turbulence, star formation and feedback by averaged quantities in a box of given size. These onezone calculations allow us to investigate the dependence on the control parameters (total gas density, metallicity, constant rate of turbulent energy production by external driving) and the coefficients of the models. In particular, we calculate the star formation efficiency for self-regulated equilibria. These equilibrium solutions are useful in their own right for a parametrization of the star forma- tion efficiency in various astrophysical applications. The full implementation as an SGS model for cosmological and galaxy-scale simulations is the goal of future work.

An outline of the proposed multi-phase model will be given in Section 2, followed by detailed descriptions of the star formation model (Section 3 ) and the model equations for the mass and energy budgets of the warm and cold phases (Section 4). In Section 5, we consider limiting cases (single phase, constant star formation rate in equilibrium). To test our model, we discuss results from one-zone calculations in Section 6, including a comparison with observations. Finally, we present our conclusions and an outlook to the application of the model in numerical simulations.

\section{OUTLINE OF THE MODEL}

The base concept of this model is to split the density in a reference volume $V=l^{3}$ (i.e. a grid cell) into a cold- and a warm-phase density with separate thermal energy budgets, as used by Springel $\&$ Hernquist (2003). The separation into two phases results from the cooling instability. In addition to the thermal energies of the cold and warm gas, the turbulent energy on the length-scale $l$ is computed. Contrary to most star formation models that are used in contemporary numerical simulations, we determine the star formation efficiency per free-fall time-scale based on local properties and processes of the turbulent multi-phase medium. To calculate the star formation efficiency, the typical length-scale of cold-gas clumps embedded in the warm neutral medium and the fraction of molecular hydrogen are important parameters. The molecular hydrogen fraction, in turn, depends on the composition and the density of the gas. To close the system of equations, we assume virial equilibrium for the cold phase, which is largely dependent on the effective pressures, i.e. the sum of thermal and turbulent pressures, of the phases. Since the turbulent pressure contribution is scale dependent, the equilibrium also depends on the clump length-scale.

In the following, quantities with subscript 'c' belong to the cold phase, those with ' $w$ ' to the warm phase, those with 's' to the star formation and those without the latter subscripts denote quantities of all the gas in the reference volume. An overview of used model specific parameters and variables is given in Table 1 .

\subsection{Specific energy variables}

The total thermal energy density $u \rho$ can be expressed as sum of the thermal energies of the cold and warm phases:

$u \rho=u_{\mathrm{c}} \rho_{\mathrm{c}}+u_{\mathrm{w}} \rho_{\mathrm{w}}$,

where fractional densities $\rho_{\mathrm{c}}$ and $\rho_{\mathrm{w}}$ are given by the masses $m_{\mathrm{w}}$ and $m_{\mathrm{c}}$ in the warm and cold phases, respectively, divided by the reference volume $V$, and $\rho=\rho_{\mathrm{c}}+\rho_{\mathrm{w}}$ is the total gas density.

The specific thermal energy of the warm phase, $u_{\mathrm{w}}$, is changed by radiative cooling and heating, the mixing of hot SN ejecta and cold gas, and turbulent dissipative heating. On the other hand, we assume that $u_{\mathrm{c}}$, the specific thermal energy of the cold phase, has a constant value, corresponding to an average temperature $T_{\mathrm{c}}=$ $50 \mathrm{~K}$ of the cold phase. Numerical simulations suggest that the isothermal approximation is reasonable for the cold phase of the ISM, because most of the gas in the cold gas is situated close to the asymptotically isothermal branch of the equilibrium curve between radiative cooling and heating (Audit \& Hennebelle 2010; Seifried, Schmidt \& Niemeyer 2011). To preserve energy conservation in our model, we account for any heating process that affects the cold gas by a transfer of a certain amount of cold gas to the warm phase.

(c) 2012 The Authors, MNRAS 421, 1838-1860 Monthly Notices of the Royal Astronomical Society (C) 2012 RAS 
Table 1. Set of model parameters and important variables.

\begin{tabular}{ll}
\hline Symbol & Description \\
\hline & \\
$l$ & Size of region \\
$\rho$ & Total mass density \\
$u_{\mathrm{c}}$ & Specific thermal energy of cold gas \\
$\Sigma$ & Rate of energy injection by the turbulent cascade \\
$I_{v}$ & Intensity of incident UV radiation field \\
& \\
& \\
$\epsilon_{\mathrm{cc}}$ & Efficiency of cold-phase evaporation by clump collisions \\
$\epsilon_{\mathrm{tt}}$ & Efficiency of turbulence production via phase separation \\
$\epsilon_{\mathrm{SN}}$ & Efficiency of turbulence production by SNe \\
$u_{\mathrm{SN}}$ & Specific energy of SN ejecta \\
$\eta$ & Turbulent velocity scaling exponent \\
$b$ & Compressive factor, describing the ratio of solenoidal and compressive turbulent modes \\
$f_{\text {loss }}$ & Fraction of mass ejected during prestellar collapse \\
$\zeta_{\mathrm{m}}$ & Fraction of newly build-up metals in SN ejecta \\
$x_{\mathrm{Lyc}}$ & Energy deposited in gas per absorbed Lyc photon \\
& \\
$u_{\mathrm{W}}$ & $\quad$ Specific thermal energy of warm gas \\
$e_{\mathrm{t}}$ & Specific turbulent energy \\
$\rho_{\mathrm{W}}$ & Fractional density of warm gas \\
$\rho_{\mathrm{w}, \mathrm{pa}}$ & Average density in the warm phase \\
$\rho_{\mathrm{c}}$ & Fractional density of cold gas \\
$\rho_{\mathrm{c}, \mathrm{pa}}$ & Average density in the cold phase \\
$\rho_{\mathrm{S}}$ & Averaged stellar mass density \\
$f_{\mathrm{c}, \mathrm{H}_{2}}$ & Mass fraction of shielded molecular gas in the cold phase \\
$l_{\mathrm{c}}$ & Size of cold clumps \\
$\mathrm{SFR}$ & Fraction of shielded molecular gas converted into stars per respective free-fall time \\
$\varepsilon_{\mathrm{ff}}$ & Fraction of total density converted into stars per respective free-fall time \\
$Z$ & Mass fraction of heavy elements \\
\hline &
\end{tabular}

Apart from the thermal energy, we assume that the gas in both phases has a certain specific turbulent energy $e_{\mathrm{t}}$ that corresponds to nearly isotropic random motions on length-scales smaller than the size $l$ of the reference volume. An exact definition of $e_{\mathrm{t}}$ will be given on the basis of a decomposition of the fluid-dynamical equation in scale space.

\subsection{Density variables and effective pressure of the gas phases}

Since each phase fills only a fraction of the total volume $V$, we define the average densities within the phases, $\rho_{\mathrm{c}, \mathrm{pa}}$ and $\rho_{\mathrm{w}, \mathrm{pa}},{ }^{1}$ by the identities

$m_{\mathrm{c}}=\rho_{\mathrm{c}, \mathrm{pa}} V_{\mathrm{c}}=\rho_{\mathrm{c}} V$,

$m_{\mathrm{w}}=\rho_{\mathrm{w}, \mathrm{pa}} V_{\mathrm{w}}=\rho_{\mathrm{w}} V$.

where $V_{\mathrm{c}}$ is the volume occupied by the cold gas phase, and $V_{\mathrm{w}}=$ $V-V_{\text {c. }}$

Quantities such as the star formation rate, the molecular fraction in the cold phase and the cooling rate depend on $\rho_{\mathrm{w}, \mathrm{pa}}$ and $\rho_{\mathrm{c}, \mathrm{pa}}$. To determine $V_{\mathrm{c}}$, it would be necessary to know the structure of the two-phase medium on length-scales smaller than $l$. In principle, one could parametrize the cold gas fraction $V_{\mathrm{c}} / V$ from small-scale simulations of thermally bistable turbulence (Seifried et al. 2011). However, because of the high sensitivity of the thermal instability

${ }^{1}$ The subscript 'pa' means 'phase average'.

(c) 2012 The Authors, MNRAS 421, 1838-1860

Monthly Notices of the Royal Astronomical Society (C) 2012 RAS on the environment (boundary conditions, gas density, etc.), it is not obvious how to relate the parameters of such idealized simulations to the local properties of a grid cell in large-scale simulations.

A much simpler approach is to assume that the cold gas is nearly in viral equilibrium if turbulence is accounted for and that clouds of cold gas with a characteristic scale $l_{\mathrm{c}}$ are embedded in the warm phase. The effect of turbulence can be described by an effective pressure that includes both microscopic (thermal) and macroscopic (non-thermal) motions (a precise definition will be given below). For a spherical cloud of density $\rho_{\mathrm{c}, \mathrm{pa}}$ and size $l_{\mathrm{c}}$, the generalized virial theorem implies the equilibrium condition

$3 P_{\mathrm{c}, \text { eff }}-\frac{\pi}{5} G \rho_{\mathrm{c}, \mathrm{pa}}^{2} l_{\mathrm{c}}^{2}-3 P_{\mathrm{w}, \mathrm{eff}} \simeq 0$,

where the effective pressure of the warm phase is subtracted as external pressure (see section 14.1 in Lequeux 2005). Since the turbulent pressure depends on the length-scale, $P_{\mathrm{c}, \text { eff }}$ and $P_{\mathrm{w}, \text { eff }}$ are also functions of $l_{\mathrm{c}}$. In principle, this equation could be used to determine the length-scale $l_{\mathrm{c}}$. It turns out, however, that the resulting system of equations is generally not well posed, meaning that no solutions exist for regions in the parameter space that definitely could be swept through in numerical simulations. As a consequence, either the relatively simple model with a single, characteristic length-scale $l_{\mathrm{c}}$ has to be abandoned or the assumption of virial equilibrium as formulated above has to be loosened. A multi-scale model might eventually result from recent theoretical developments (P. Hennebelle, private communication). In this article, we choose the second option and investigate its consequences. Typically, structures satisfying 


\section{2}

\section{H. Braun and W. Schmidt}

equation (4) are not gravitationally bound. The dominant contributions come from the effective pressure, and these structures are held together by the pressure that is exerted by the surrounding warm gas. For this reason, the gravitational energy term can be neglected, and we obtain an approximate effective pressure balance:

$P_{\mathrm{w}, \text { eff }} \stackrel{!}{=} P_{\mathrm{c}, \text { eff }}$,

On the average, the turbulent pressure significantly contributes to the support of the cold gas against gravity. In order to connect the properties of the cold phase to the star formation rate, we assume that localized regions exist in the cold phase, where weak turbulent pressure support persists over sufficiently long periods of time so that the gas can collapse. The existence of such regions is a consequence of the intermittency of turbulence. The critical size of these regions is roughly given by the thermal Jeans length,

$\lambda_{\mathrm{J}, \mathrm{c}}=c_{\mathrm{c}}\left(\frac{\pi}{\gamma G \rho_{\mathrm{c}, \mathrm{pa}}}\right)^{1 / 2}=\left(\frac{\pi(\gamma-1) u_{\mathrm{c}}}{G \rho_{\mathrm{c}, \mathrm{pa}}}\right)^{1 / 2}$,

where $c_{\mathrm{c}}=\left[\gamma(\gamma-1) u_{\mathrm{c}}\right]^{1 / 2}$ is the speed of sound in the cold gas, $\gamma$ is the polytropic equation of state parameter and $G$ is the gravitational constant. Thus, we define the length-scale $l_{\mathrm{c}}$ by

$l_{\mathrm{c}}=\lambda_{\mathrm{J}, \mathrm{c}}$.

The effective pressure equilibrium (5) and the length-scale (7) really have a complementary meaning. While the former statistically accounts for the overall effect of turbulence, the latter specifies a typical size of locally collapsing structures in the cold phase. In a certain sense, this corresponds to the fact that molecular clouds do not collectively collapse although their mass is much greater than the thermal Jeans mass, while gravitationally unstable cores are formed locally (Mac Low \& Klessen 2004)

From the effective pressure balance (5) between the phases follows the ratio

$\frac{\rho_{\mathrm{c}, \mathrm{pa}}}{\rho_{\mathrm{w}, \mathrm{pa}}}=r_{\mathrm{w}}:=\frac{\sigma_{\mathrm{w}, \mathrm{eff}}}{\sigma_{\mathrm{c}, \mathrm{eff}}}$,

where $\sigma_{\mathrm{w}, \text { eff }}$ and $\sigma_{\mathrm{c} \text {,eff }}$ are functions of the internal energies $u_{\mathrm{w}}$ and $u_{\mathrm{c}}$, and the turbulent energy $e_{\mathrm{t}}$. Combining equations (2) and (8), we can express the phase densities and volumes in terms of the fractional densities and the specific pressures:

$\rho_{\mathrm{c}, \mathrm{pa}}=r_{\mathrm{w}} \rho_{\mathrm{w}}+\rho_{\mathrm{c}}, \quad V_{\mathrm{c}}=\frac{\rho_{\mathrm{c}} V}{\rho_{\mathrm{c}}+r_{\mathrm{w}} \rho_{\mathrm{w}}}$,

$\rho_{\mathrm{w}, \mathrm{pa}}=r_{\mathrm{w}}^{-1} \rho_{\mathrm{c}}+\rho_{\mathrm{w}}, \quad V_{\mathrm{w}}=\frac{\rho_{\mathrm{w}} V}{\rho_{\mathrm{w}}+r_{\mathrm{w}}^{-1} \rho_{\mathrm{c}}}$

Furthermore, we define the stellar density $\rho_{\mathrm{s}}$ to be the stellar mass within the reference volume $V$ divided by that volume:

$\rho_{\mathrm{s}}=\frac{m_{\mathrm{s}}}{V}$.

Numerical simulations of forced turbulence in thermally bistable gas indicate that the specific turbulent energy is nearly isotropic and uniformly distributed among the phases (Seifried et al. 2011). Thus, the turbulent velocity dispersion within the cold gas can be related to the turbulent energy on the length-scale $l$ via the power law

$3 \sigma_{\mathrm{c}}^{2}=2 e_{\mathrm{t}}\left(\frac{l_{\mathrm{c}}}{l}\right)^{2 \eta}$.

The scaling exponent $\eta$ is constrained by $1 / 3 \leq \eta \leq 1 / 2$, where the lower and upper bounds correspond to Kolmogorov and Burgers scaling, respectively. This scaling law is consistent with the observed $\sigma_{\mathrm{c}}$-scaling relation (see, for example, Larson 1981).
With the above definition, the effective pressure of the cold gas on the length-scale $l_{\mathrm{c}}$ is given by (see SF11)

$$
\begin{aligned}
P_{\mathrm{c}, \mathrm{eff}} & =\rho_{\mathrm{c}, \mathrm{pa}} \sigma_{\mathrm{c}, \mathrm{eff}} \equiv \rho_{\mathrm{c}, \mathrm{pa}}\left(\frac{c_{\mathrm{c}}^{2}}{\gamma}+\sigma_{\mathrm{c}}^{2}\right) \\
& =(\gamma-1) \rho_{\mathrm{c}, \mathrm{pa}} u_{\mathrm{c}}\left(1+\frac{\gamma}{3} \mathcal{M}_{\mathrm{c}}^{2 \eta}\right),
\end{aligned}
$$

where $\mathcal{M}_{\mathrm{c}}=\sqrt{3} \sigma_{\mathrm{c}} / c_{\mathrm{c}}$ is the root mean square (rms) Mach number of turbulence in the cold phase. The turbulent pressure $P_{\mathrm{w}, \text { eff }}$ is given by an analogous expression, with cold-phase quantities replaced by the corresponding quantities in the warm phase. With these definitions, the variables $l_{\mathrm{c}}, \rho_{\mathrm{c}, \mathrm{pa}}$ and $\rho_{\mathrm{w}, \mathrm{pa}}$ can be determined solving equations (9), (10) and (7) iteratively.

\subsection{Gas composition variables}

The chemical composition of the gas is for simplicity, as we do not track individual species, identified by its mass fraction of heavy elements $Z$. Given the mass fraction of helium $\mathrm{Y}_{\odot}$ at solar metallicity $\mathrm{Z}_{\odot}$ and its primordial value $Y_{\text {prim }}, Y$ at metallicity $Z$ is assumed to be $Y=Y_{\text {prim }}+\left(\mathrm{Y}_{\odot}-Y_{\text {prim }}\right) Z$. Then the total mass fraction of hydrogen $X$ is given by $X=1-Y-Z$. If the gas is neutral, but not molecular, which is approximately true in the warm phase and in the cold-phase gas outside of molecular cores, the mean molecular weight $\mu$ is given by

$\left(m_{\mathrm{H}} \mu\right)^{-1}=X m_{\mathrm{H}}^{-1}+Y m_{\mathrm{He}}^{-1}+Z m_{Z}^{-1}$,

where $m_{\mathrm{H}}$ and $m_{\mathrm{He}}$ are the atomic masses of hydrogen and helium, respectively, and $m_{Z}$ is the average atomic mass of the heavier elements. Within the molecular cores of the cold phase we assume the gas to be fully molecular.

\section{STAR FORMATION}

Following KMT09, cold gas is converted into stars at a rate that depends on the mass of molecular hydrogen in the reference volume $\left(m_{\mathrm{H}_{2}}=f_{\mathrm{c}, \mathrm{H}_{2}} \rho_{\mathrm{c}} V\right.$, where $f_{\mathrm{c}, \mathrm{H}_{2}}$ is the molecular hydrogen fraction in the cold gas phase):

$\dot{\rho}_{\mathrm{s}}=\frac{\left(1-f_{\text {loss }}\right) f_{\mathrm{c}, \mathrm{H}_{2}} \rho_{\mathrm{c}}}{t_{\mathrm{s}}}$.

We define the star formation time-scale $t_{\mathrm{s}}$ by

$t_{\mathrm{s}}=\frac{t_{\mathrm{c}, \mathrm{ff}}}{\mathrm{SFR}_{\mathrm{c}, \mathrm{ff}}}$,

where the free-fall time-scale in the cold gas is given by the phaseaverage (not the fractional) density:

$t_{\mathrm{c}, \mathrm{ff}}^{2}=\frac{3 \pi}{32 G \rho_{\mathrm{c}, \mathrm{pa}}}$

and $\mathrm{SFR}_{\mathrm{c}, \mathrm{ff}}$ is the dimensionless star formation rate per free-fall time $t_{\mathrm{c}, \text { ff }}$. Not all the mass in collapsing prestellar cores eventually ends up in stars. A fraction $f_{\text {loss }} \simeq 0.5, \ldots, 0.7$ of mass is ejected during prestellar collapse (e.g. Matzner \& McKee 2000; Hennebelle \& Chabrier 2008; Chabrier \& Hennebelle 2010). We account for the mass ejection by correcting the star formation rate by the factor $\left(1-f_{\text {loss }}\right)$ in equation $(25)$.

To calculate $\mathrm{SFR}_{\mathrm{c}, \mathrm{ff}}$, KMT09 derive a parametrization in terms of the gas column density, which reproduces important observational results from recent high-resolution surveys. These data also imply that the star formation is tightly correlated with the density of molecular hydrogen. This is the reason for including the factor $f_{\mathrm{c}, \mathrm{H}_{2}}$ 
in equation (16). On the other hand, Glover \& Clark (2012) questioned a causal relationship between the star formation rate and the molecular hydrogen fraction. They argue that the observed correlation results form the necessity of effective shielding of star-forming regions from the interstellar radiation field. But this is in essence the effect that KMT09 describe with their model. For this reason, we also include the molecular hydrogen fraction as a coefficient in the expression for the star formation rate.

KMT09 implicitly account for the turbulent energy by assuming that molecular clouds are virialized. In addition, the molecular cloud mass is determined by setting the Toomre stability parameter equal to unity. In Section 2.2 we determine the mean cold-gas density $\rho_{\mathrm{c} \text {,pa }}$ from an effective pressure balance, and we introduce a characteristic scale $l_{\mathrm{c}}$ that is given by the thermal Jeans mass for this density. Since turbulence in the cold phase is generally supersonic, the local density of the gas greatly fluctuates. Therefore, we consider a statistical ensemble of overdense structures on the length-scale $l_{\mathrm{c}}$. For convenience, we call these structures clumps. The greater the overdensity relative to $\rho_{\mathrm{c} \text {, pa }}$, the smaller is the critical density for gravitational collapse. For a given statistical distribution of density fluctuations, which we assume to be lognormal, the dimensionless star formation rate $\mathrm{SFR}_{\mathrm{c}, \mathrm{ff}}$ then can be calculated as proposed by PN11.

\subsection{Star formation efficiency}

By assuming a one-dimensional $\mathrm{rms}$ turbulent velocity dispersion $\sigma_{\mathrm{c}}$, the virial parameter of a clump at the mean density $\rho_{\mathrm{c}, \mathrm{pa}}$ is given by Bertoldi \& McKee (1992)

$\alpha_{\mathrm{vir}}=\frac{15}{\pi G \rho_{\mathrm{c}, \mathrm{pa}}}\left(\frac{\sigma_{\mathrm{c}}}{l_{\mathrm{c}}}\right)^{2} \propto\left(\frac{t_{\mathrm{c}, \mathrm{ff}}}{t_{\mathrm{c}, \mathrm{dyn}}}\right)^{2}$,

where $t_{\mathrm{c}, \text { dyn }}=l_{\mathrm{c}} /\left(\sqrt{3} \sigma_{\mathrm{c}}\right)$ is the dynamical time-scale. Since the statistical ensemble of clumps has to encompass the whole cold phase, not only regions of weak turbulent support, the rms velocity dispersion is given by the turbulent energy scaled down to the lengthscale $l_{\mathrm{c}}$. Hence, by substituting the scaling law (equation 12), the virial parameter can be expressed as

$\alpha_{\mathrm{vir}}=\frac{10}{\pi G \rho_{\mathrm{c}, \mathrm{pa}}} \frac{e_{\mathrm{t}}}{l_{\mathrm{c}}^{2(1-\eta)} l^{2 \eta}}$.

PN11 argue that the overdensity in compressed shock layers is proportional to the square of the Mach number, $\mathcal{M}_{\mathrm{c}}^{2}$ (see Section 2.2 for the definition of $\mathcal{M}_{\mathrm{c}}$ ). By applying the Jeans criterion for the gravitational collapse of a compressed region within the cold gas phase, it follows that the critical overdensity ratio $x_{\text {crit }}=\rho_{\mathrm{c}, \text { crit }} / \rho_{\mathrm{c}, \mathrm{pa}}$ is proportional to $\alpha_{\mathrm{vir}} \mathcal{M}_{\mathrm{c}}^{2}$ :

$$
\begin{aligned}
x_{\text {crit }} & =0.0371 \alpha_{\text {vir }} \mathcal{M}_{\mathrm{c}}^{2} \\
& =\frac{0.0742}{G \gamma(\gamma-1) \rho_{\mathrm{c}, \mathrm{pa}} u_{\mathrm{c}}} \frac{e_{\mathrm{t}}^{2}}{l_{\mathrm{c}}^{2(1-2 \eta)} l^{4 \eta}} .
\end{aligned}
$$

The constant of proportionality in the above equation is fixed by the definition of the Bonnor-Ebert radius (see PN11). For MHD turbulence, PN11 show that $x_{\text {crit }}$ differs by a factor $\beta$ that specifies the ratio of thermal to magnetic pressures.

Since $u_{\mathrm{c}}=$ const., the variation of the critical density $\rho_{\text {crit }}=$ $x_{\text {crit }} \rho_{\text {c,pa }}$ is solely determined by the second factor in equation (20). For the two limiting cases of Kolmogorov and Burgers scaling, we obtain

$$
\rho_{\text {crit }} \propto \begin{cases}e_{\mathrm{t}}^{2} l_{\mathrm{c}}^{-2 / 3} l^{-4 / 3} & \text { if } \eta=1 / 3, \\ e_{\mathrm{t}}^{2} l^{-2} & \text { if } \eta=1 / 2 .\end{cases}
$$

(c) 2012 The Authors, MNRAS 421, 1838-1860

Monthly Notices of the Royal Astronomical Society (C) 2012 RAS

\section{A model of the turbulent multi-phase ISM}

If the warm phase dominates $\left(l_{\mathrm{c}} \ll l\right)$, then $\eta \approx 1 / 3$ because, averaged over a region of size $l$, turbulence is mostly subsonic. From the scaling law $e_{\mathrm{t}} \propto l^{2 / 3}$, it follows that $\rho_{\text {crit }} \propto l_{\mathrm{c}}^{-2 / 3}$. The scaling behaviour of the critical density follows from the steeper decrease of self-gravity with the clump size relative to the lower turbulent energy on smaller length-scales. The assumption of Kolmogorov scaling is not at odds with supersonic turbulence within the clumps, because supersonic scaling applies to length-scales $l \lesssim$ $l_{\mathrm{c}}$ only. However, the assumption of a uniform velocity dispersion between both phases might break down for large $\mathcal{M}_{\mathrm{c}}$. On the other hand, if the cold gas phase fills most of the volume $V=l^{3}, \eta$ assumes a value greater than $1 / 3$, depending on $\mathcal{M}_{\mathrm{c}}$. In the limit of high turbulent Mach numbers, Burgers scaling $\left(e_{\mathrm{t}} \propto l\right)$ implies that $\rho_{\text {crit }}$ becomes nearly scale invariant. ${ }^{2}$ In this case, however, a potential problem is that the turbulent pressure within the overdense cores (i.e. on the length-scale of the shock-compressed layer, which is small compared to $l_{\mathrm{c}}$ ) can exceed the thermal pressure. Consequently, the model overestimates the mass that can form star in the limit of strongly supersonic clumps of size $l_{\mathrm{c}} \sim l$. Applying the model in numerical simulations, it has to be ensured that this case is sufficiently rare.

As in KM05, the mass fraction per free-fall time that is converted into stars is derived from the formula

$\mathrm{SFR}_{\mathrm{c}, \mathrm{ff}}=\int_{x_{\text {crit }}}^{\infty} x p(x) \mathrm{d} x$,

where $p(x)$ is the probability density function (pdf) of the mass density, and $x=\rho_{\mathrm{c}, \text { loc }} / \rho_{\mathrm{c}, \mathrm{pa}}$ is the ratio of the local and mean densities in the cold phase.

For isothermal gas, the pdf of the gas density is approximately lognormal (e.g. Kritsuk et al. 2007; Federrath, Klessen \& Schmidt 2008):

$p(x) \mathrm{d} x=\frac{x^{-1}}{\left(2 \pi \sigma^{2}\right)^{1 / 2}} \exp \left[-\frac{(\ln (x)-\langle\ln (x)\rangle)^{2}}{2 \sigma^{2}}\right] \mathrm{d} x$,

where $\sigma^{2}=-\langle\ln (x)\rangle$ is the standard deviation of logarithmic overdensity. Lognormal fits to the density pdfs from the numerical simulations suggest the following empirical relation between $\sigma$ and the sonic Mach number:

$\sigma^{2} \approx \ln \left(1+b^{2} \mathcal{M}_{\mathrm{c}}^{2}\right)$.

As shown by Federrath et al. (2010), the parameter $b$ depends on the mixture of solenoidal and compressive forcing modes, which is specified by the weighing parameter $\zeta$ of the Helmholtz decomposition of the forcing modes:

$b=\frac{1}{3}+\frac{2}{3}\left(\frac{(1-\zeta)^{2}}{1-2 \zeta+3 \zeta^{2}}\right)^{3}$.

For solenoidal (divergence-free) forcing, $\zeta=1$. On the other hand, $\zeta=0$ for compressive (rotation-free) forcing. Substituting the lognormal pdf (22) into equation (21), the dimensionless star formation rate is given by

$\mathrm{SFR}_{\mathrm{c}, \mathrm{ff}}=\frac{1}{2}+\frac{1}{2} \operatorname{erf}\left[\frac{\sigma^{2}-2 \ln \left(x_{\text {crit }}\right)}{2^{3 / 2} \sigma}\right]$.

Numerical simulations of self-gravitating turbulence (e.g. Klessen 2001; Federrath et al. 2008; Cho \& Kim 2011; Kritsuk, Norman \& Wagner 2011) show changes of the high-density tail of

${ }^{2}$ In this case, the coefficient following from the assumption of spherical clumps would not be appropriate, but the scaling remains unaffected. 


\section{4}

\section{H. Braun and W. Schmidt}

the pdf, which affect $\mathrm{SFR}_{\mathrm{c}, \mathrm{ff}}$. They find a power-law tail, which is associated with self-gravitating cores. Simulations by BallesterosParedes et al. (2011) suggest that in a star-forming cloud the pdf only develops a power-law tail at high densities over periods of $\gtrsim 10 \mathrm{Myr}$, while the contribution of self-gravitating cores to the pdf is negligible in the earlier phase and, thus, the shape is close to lognormal Since the model of PN11 for $\mathrm{SFR}_{\mathrm{c}, \mathrm{ff}}$ is conceptually based on the turbulence-dominated phase, it is consistent to assume a lognormal pdf. An advanced formulation of the model might also account for the later power-law phase, but this would also require substantial modifications in the ansatz for $\mathrm{SFR}_{\mathrm{c}, \mathrm{ff}}$. We do not consider this in the present work.

Furthermore, we assume a distribution of clump overdensities that is determined by the global rms turbulent energy to estimate the fraction of collapsing gas in our PN11-like calculation of the star formation efficiency. This amounts to a separation of the density and velocity fluctuations. Strictly, the fraction of cold gas that can collapse should be calculated from the distributions of both the density and the turbulent velocity fluctuations. As Hennebelle \& Chabrier (2008) have already pointed out, however, this is far from trivial, and we do not attempt to solve this problem here.

\subsection{Molecular hydrogen fraction}

The formation of $\mathrm{H}_{2}$ molecules and their radiative destruction are mostly heating processes. Because both rates are enhanced with density, overdense regions in a clump of cool but not molecular gas may be dispersed by this heating effect, before they possibly collapse gravitationally. So, knowing the fraction of molecular dominated gas in a clump, as a tracer for the fraction of gas that is not affected by effective radiation induced heating, is essential to correctly estimate the star formation rate. The fraction of molecular-dominated gas in a cold clump is strongly dependent on shielding radiation, which dissociates $\mathrm{H}_{2}$-molecules easily, from its inner parts. Here we use a Stömgren-like approach similar to that McKee \& Krumholz (2010) used. In low-metallicity environments, this approach may lead to too high molecular fraction estimates, as reaction rates are too slow to establish dissociation equilibrium on short time-scales (Krumholz \& Gnedin 2011). But, for our purpose this is fair enough, as we do not intend to track a whole chemical network of several species. Moreover a simple chemical network model, like that of Gnedin et al. (2009), may have weaknesses, as it particularly in the case of large $\mathrm{H}_{2}$ fractions, which is of particular interest when looking at star formation, overestimates further $\mathrm{H}_{2}$ production (Micic et al. 2012). Apart from that, this approach is not designed to compute the total fraction of molecular gas but the fraction that is molecular dominated, as we totally neglect molecular hydrogen in radiation-dominated areas. Nevertheless, we compare the results of this approach to observations in Section 6.4. Assuming spherical clouds with diameter $l_{\mathrm{c}}$, one needs to calculate the radius $l_{\mathrm{c}, \mathrm{H}_{2}}$, at which the production rate $R_{\mathrm{H}_{2} \text {,prod }}$ of $\mathrm{H}_{2}$ becomes greater than its destruction rate $R_{\mathrm{H}_{2} \text {,diss }}$.

The molecular fraction of cold gas then can be expressed as the ratio of the molecular volume in a clump $\propto l_{\mathrm{c}, \mathrm{H}_{2}}^{3}$ and the total volume of the clump $\propto\left(l_{\mathrm{c}} / 2\right)^{3}$ :

$f_{\mathrm{c}, \mathrm{H}_{2}}=\left(\frac{2 l_{\mathrm{c}, \mathrm{H}_{2}}}{l_{\mathrm{c}}}\right)^{3}$,

where $l_{\mathrm{c}, \mathrm{H}_{2}}$ meets the condition

$$
\frac{R_{\mathrm{H}_{2}, \text { prod }}}{R_{\mathrm{H}_{2}, \text { diss }}\left(\hat{d}_{\mathrm{c}, \mathrm{H}_{2}}\right)}=1 \text {, }
$$

where $\hat{d}_{c, \mathrm{H}_{2}}$ is the effective shielding layer thickness $\hat{d}$, at a position inside the clump, where equation (27) is true. The $\mathrm{H}_{2}$ production and destruction rates depending on $\hat{d}$, assuming extinction of dissociating radiation of the outer regions of the cold clump is not sufficient, are given by (see Gnedin et al. 2009)

$R_{\mathrm{H}_{2}, \mathrm{prod}}=\frac{\rho_{\mathrm{c}, \mathrm{pa}}^{2}}{m_{\mathrm{H}}^{2}} C_{\rho} X\left(\frac{Z}{\mathrm{Z}_{\odot}} r_{\mathrm{H}_{2}, \mathrm{p}, \mathrm{s}}+X r_{\mathrm{H}_{2}, \mathrm{p}, \mathrm{g}}\right)$

and

$R_{\mathrm{H}_{2}, \text { diss }}(\hat{d})=I_{\nu} S_{\text {dust }} S_{\mathrm{H}_{2}} \frac{\rho_{\mathrm{c}, \mathrm{pa}}}{m_{\mathrm{H}}} r_{\mathrm{H}_{2}, \mathrm{~d}}$,

respectively, where $C_{\rho}=\mathrm{e}^{\sigma^{2}}$ is the clumping factor (Gnedin et al. 2009) with $\sigma$ as defined in equation (23), $\mathrm{Z}_{\odot}$ is the solar metal fraction, $r_{\mathrm{H}_{2}, \mathrm{p}, \mathrm{s}}$ is the $\mathrm{H}_{2}$ formation rate on dust surfaces, $r_{\mathrm{H}_{2}, \mathrm{p}, \mathrm{g}}$ is the $\mathrm{H}_{2}$ formation rate in the gaseous phase, $r_{\mathrm{H}_{2}, \mathrm{~d}}$ is the radiative dissociation rate, $I_{v}$ is the intensity of the homogeneous isotropic dissociating radiation field relative to the Draine (1978) field, $S_{\text {dust }}$ and $S_{\mathrm{H}_{2}}$ are the shielding factors due to dust and $\mathrm{H}_{2}$ itself (see Glover \& Mac Low 2007 or Draine \& Bertoldi 1996)

$S_{\text {dust }}=\exp \left(-\sigma_{\text {dust }} \frac{Z}{Z_{\odot}} \rho_{\text {c,pa }} \hat{d}\right)$,

$S_{\mathrm{H}_{2}}=\frac{1-\omega_{\mathrm{H}_{2}}}{(1+x)^{2}}+\frac{\omega_{\mathrm{H}_{2}}}{(1+x)^{1 / 2}} \exp \left(-\sigma_{\mathrm{H}_{2}}(1+x)^{1 / 2}\right)$

where

$x=f_{\mathrm{c}, \mathrm{H}_{2}, 0} \rho_{\mathrm{c}, \mathrm{pa}} \hat{d} /\left(m_{\mathrm{H}} \kappa\right)$,

with $\kappa=5 \times 10^{14} \mathrm{~cm}^{-2}$ and

$f_{\mathrm{c}, \mathrm{H}_{2}, 0}=\max \left(f_{\mathrm{c}, \mathrm{H}_{2}, \min }, R_{\mathrm{H}_{2}, \operatorname{prod}} / R_{\mathrm{H}_{2}, \text { diss }}(\hat{d}=0)\right)$

$\left(f_{\mathrm{c}, \mathrm{H}_{2}, \text { min }} \approx 10^{-5}\right.$ is the minimum molecular fraction in radiationdominated regions of the cold phase).

In the centre of the spherical clump of diameter $l_{\mathrm{c}}$, the shielding layer has the same thickness for all directions, i.e. $\hat{d}=l_{\mathrm{c}} / 2$. So if

$R_{\mathrm{H}_{2}, \text { prod }} / R_{\mathrm{H}_{2} \text {,diss }}\left(\hat{d}=l_{\mathrm{c}} / 2\right) \leq 1$

holds, there is no molecular core in the clump, and thus $f_{\mathrm{c}, \mathrm{H}_{2}}=0$. Otherwise there is one, which is then assumed to effectively block all dissociating radiation, trying to pass it. For a given position of scaled distance $\lambda=2 \hat{l} / l_{\mathrm{c}}$ from the centre outside the molecular core $\left(l_{\mathrm{c}, \mathrm{H}_{2}}<\hat{l}\right)$ the scaled effective absorption layer thickness $\delta=2 \hat{d} / l_{\mathrm{c}}$ is given by the mean of the absorption layer thicknesses over all sky $O$, but the solid angle of the molecular core $S$,

$\delta(\lambda)=\int_{O \backslash S(\lambda)} \delta^{\prime}(\Omega) g(\Omega) \mathrm{d} \Omega / \int_{O \backslash S(\lambda)} g(\Omega) \mathrm{d} \Omega$,

weighted by the fraction of transmitted radiation, which is approximated by $g=\mathrm{e}^{-\delta^{\prime}}$. The number of photons, which can possibly reach that position, is due to the cores shadow reduced by

$I_{\nu, \text { shadow }}(\lambda)=\frac{I_{v}}{4 \pi} \int_{S(\lambda)} \mathrm{d} \Omega$.

At the edge of the core $\lambda_{\mathrm{c}, \mathrm{H}_{2}}=2 l_{\mathrm{c}, \mathrm{H}_{2}} / l_{\mathrm{c}}$ half the sky is obscured (i.e. $\left.I_{v \text {, shadow }}\left(\lambda_{\mathrm{c}, \mathrm{H}_{2}}\right)=I_{v} / 2\right)$. After integrating/substituting out all angular dependencies, we have

$\delta_{\mathrm{c}, \mathrm{H}_{2}}\left(\lambda_{\mathrm{c}, \mathrm{H}_{2}}\right)=\int_{\delta_{\min }}^{\delta_{\max }} \delta^{\prime} g\left(\delta^{\prime}\right) \mathrm{d} \delta^{\prime} / \int_{\delta_{\min }}^{\delta_{\max }} g\left(\delta^{\prime}\right) \mathrm{d} \delta^{\prime}$,

with $\delta_{\min }=1-\lambda_{\mathrm{c}, \mathrm{H}_{2}}, \delta_{\max }=\sqrt{1-\lambda_{\mathrm{c}, \mathrm{H}_{2}}^{2}}$ and

$g\left(\delta^{\prime}\right)=4 \pi \delta^{\prime 2} \mathrm{e}^{-\delta^{\prime}}\left(1-\left(\frac{1+\lambda_{\mathrm{c}, \mathrm{H}_{2}}^{2}-\delta^{\prime 2}}{2 \lambda_{\mathrm{c}, \mathrm{H}_{2}}}\right)^{2}\right)^{-1 / 2}$. 
If equations (28) to (33) are substituted into equation (27) and using $I_{\nu \text {,shadow }}\left(\lambda_{\mathrm{c}, \mathrm{H}_{2}}\right)$ instead of $I_{v}$, one obtains a transcendent equation for $z \equiv(x+1)^{1 / 2}$ :

$C=C(z) \equiv\left(\frac{1-\omega_{\mathrm{H}_{2}}}{z^{4}}+\frac{\omega_{\mathrm{H}_{2}}}{z} \mathrm{e}^{-\sigma_{\mathrm{H}_{2}} z}\right) \mathrm{e}^{-D\left(z^{2}-1\right)}$,

where

$C \equiv \frac{\rho_{\mathrm{c}, \mathrm{pa}} C_{\rho}}{I_{\nu, \text { shadow }} r_{\mathrm{H}_{2}, \mathrm{~d}} m_{H}}\left(\frac{Z r_{\mathrm{H}_{2}, \mathrm{p}, \mathrm{s}}}{\mathrm{Z}_{\odot}}+X r_{\mathrm{H}_{2}, \mathrm{p}, \mathrm{g}}\right)$,

$D \equiv \frac{\sigma_{\text {dust }} Z \kappa}{f_{\mathrm{H}_{2}, 0} \mathrm{Z}_{\odot}}$,

$E \equiv \frac{2 \kappa m_{\mathrm{H}}}{l_{\mathrm{c}} \rho_{\mathrm{c}, \mathrm{pa}} f_{\mathrm{H}_{2}, 0}}$.

Equation (39) has a single solution for every given $C$, but only solutions in the range of $z \in\left[1, \ldots, z_{\max }\right.$ [are allowed, as $l_{\mathrm{c}, \mathrm{H}_{2}}$ would be greater than $l_{\mathrm{c}} / 2$ if $z<1$ and $^{3} l_{\mathrm{c}, \mathrm{H}_{2}} \leq 0$ if $z \geq z_{\max } \equiv\left(1+E^{-1}\right)^{1 / 2}$.

As $\delta_{\mathrm{c}, \mathrm{H}_{2}}\left(\lambda_{\mathrm{c}, \mathrm{H}_{2}}\right)$ is bijective for $\lambda_{\mathrm{c}, \mathrm{H}_{2}} \in[0, \ldots, 1]$, we can use its inverse $\lambda_{\mathrm{c}, \mathrm{H}_{2}}\left(\delta_{\mathrm{c}, \mathrm{H}_{2}}\right)$ to compute the molecular fraction

$f_{\mathrm{c}, \mathrm{H}_{2}}= \begin{cases}0 & \text { if equation } \\ \left\langle\lambda_{\mathrm{c}, \mathrm{H}_{2}}\left(1-\left[z^{2}-1\right] E\right)\right\rangle^{3} & \text { if } 1<z, \\ 1 & \text { else. }\end{cases}$

\section{EVOLUTIONARY EQUATIONS}

\subsection{Exchange of mass between the phases}

The effective growth rate of the stellar mass density is given by

$\dot{\rho}_{\mathrm{s}, \text { eff }}=\dot{\rho}_{\mathrm{s}}-\dot{\rho}_{\mathrm{s}, \mathrm{fb}}$

where the star formation rate $\dot{\rho}_{\mathrm{s}}$ is defined in Section 3 , and $\dot{\rho}_{\mathrm{s}, \mathrm{fb}}$ is the rate at which gas is returned to the warm phase via core collapse supernovae.

In our model, $\dot{\rho}_{\mathrm{s}, \mathrm{fb}}$ is determined by a convolution of the past star formation rate $\dot{\rho}_{\mathrm{s}}\left(t-t^{\prime}\right)$ and the stellar IMF $\mathrm{d} N_{*} / \mathrm{d} m_{*}$ times the initial stellar mass $m_{*}$ :

$\dot{\rho}_{\mathrm{s}, \mathrm{fb}}(t)=\int_{t_{\mathrm{b}}}^{t_{\mathrm{e}}} \dot{\rho}_{\mathrm{s}}\left(t-t^{\prime}\right) \frac{1}{M_{*}} \frac{\mathrm{d} N_{*}}{\mathrm{~d} m_{*}} \frac{\mathrm{d} m_{*}}{\mathrm{~d} t^{\prime}} \mathrm{d} t^{\prime}$,

where $m_{*}=m_{*}\left(t^{\prime}, Z\right)$ is the initial mass of a star that explodes as a supernova after a lifetime $t^{\prime}$. The integration boundaries $t_{\mathrm{b}}$ to $t_{\mathrm{e}}$ correspond to the lifetimes of 40 and $8 \mathrm{M}_{\odot}$ stars, respectively. The IMF is normalized by the mean initial mass per solar mass

$M_{*}=\frac{1}{\mathbf{M}_{\odot}} \int_{0}^{\infty} m_{*} \frac{\mathrm{d} N_{*}}{\mathrm{~d} m_{*}} \mathrm{~d} m_{*}$.

For the function $m_{*}\left(t^{\prime}, Z\right.$ ), we use a parametrization (Raiteri, Villata \& Navarro 1996) of the results computed by the Padova group (Alongi et al. 1993; Bressan et al. 1993; Bertelli et al. 1994). Furthermore, we assume the IMF by Chabrier (2001):

$\frac{\mathrm{d} N_{*}}{\mathrm{~d} m_{*}} \propto \begin{cases}m_{*}^{-1} \mathrm{e}^{-\frac{\log _{10}^{2}\left(\frac{m_{*}}{m_{*, \mathrm{c}}}\right)}{2 \sigma_{*, \mathrm{c}}^{2}}} & \text { for } 0.1 \mathrm{M}_{\odot}<m_{*}<1 \mathrm{M}_{\odot}, \\ m_{*}^{-2.3} & \text { for } 1 \mathrm{M}_{\odot}<m_{*}<125 \mathrm{M}_{\odot}, \\ 0 & \text { otherwise, }\end{cases}$

where $\sigma_{*, \mathrm{c}}=0.69$ and $m_{*, \mathrm{c}}=0.08 \mathrm{M}_{\odot}$.

${ }^{3}$ Note that the following case is already covered by an even more restrictive condition given in equation (34).

(C) 2012 The Authors, MNRAS 421, 1838-1860

Monthly Notices of the Royal Astronomical Society (C) 2012 RAS

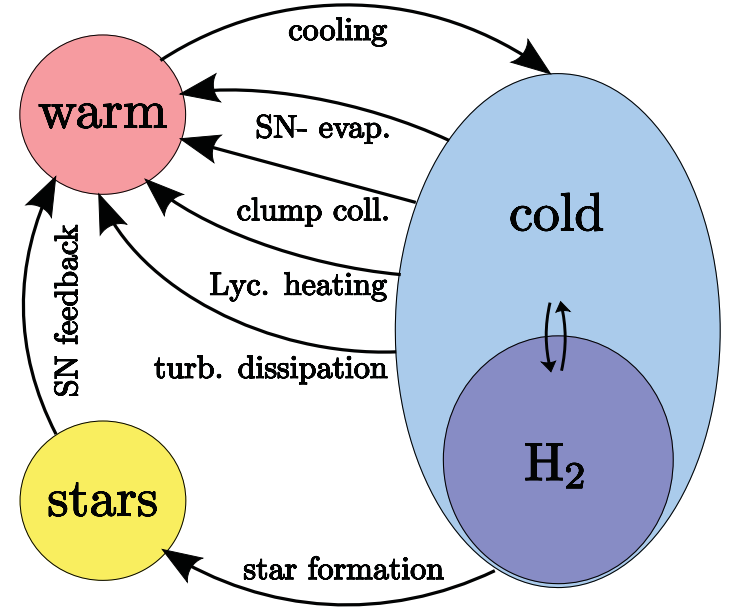

Figure 1. Scheme of the exchange of mass. The mass budgets are depicted in yellow $\left(\rho_{\mathrm{s}}\right)$, red $\left(\rho_{\mathrm{w}}\right)$ and blue $\left(\rho_{\mathrm{c}}\right)$, where the molecular mass $\left(f_{\mathrm{c}, \mathrm{H}_{2}} \rho_{\mathrm{c}}\right)$ in darker blue resides inside the cold gas mass. Arrows illustrate processes transferring mass from one to another budget.

The fraction of heavy elements in the gas increases due to SN feedback. Assuming that the metal species in the ejecta have solar relative abundances, and, that the mass fraction of newly build-up metals in the ejecta of a stellar population is independent of its initial metallicity at $\zeta_{\mathrm{m}} \approx 0.1$, we write

$\frac{\mathrm{d}(Z \rho)}{\mathrm{d} t}=-Z \dot{\rho}_{\mathrm{s}}+\left(\zeta_{\mathrm{m}} \dot{\rho}_{\mathrm{s}, \mathrm{fb}}+\int_{t_{\mathrm{b}}}^{t_{\mathrm{e}}} Z(\hat{t}) \dot{\rho}_{\mathrm{s}}(\hat{t}) \frac{1}{M_{*}} \frac{\mathrm{d} N_{*}}{\mathrm{~d} m_{*}} \frac{\mathrm{d} m_{*}}{\mathrm{~d} t^{\prime}} \mathrm{d} t^{\prime}\right)$,

with $\hat{t}=t-t^{\prime}$.

The rate of change of the fractional density of the cold phase, $\dot{\rho}_{\mathrm{c}}$, is determined by the processes that are described in the following. The first three processes are modelled as in Springel \& Hernquist (2003). For a schematic overview, see Fig. 1.

(i) Star formation reduces the mass of cold gas:

$\left.\frac{\mathrm{d} \rho_{\mathrm{c}}}{\mathrm{d} t}\right|_{\mathrm{SF}}=-\dot{\rho}_{\mathrm{s}}$.

(ii) Hot $\mathrm{SN}$ bubbles can evaporate cold clumps. Effectively, the energy that is injected by blast waves into the interstellar gas is instantaneously dissipated into heat on length-scales that are much smaller than $l$. Since we cannot resolve the mixing processes, the dissipative heating and the heat conduction on these scales, we account for these processes by an evaporation rate of the cold gas,

$\left.\frac{\mathrm{d} \rho_{\mathrm{c}}}{\mathrm{d} t}\right|_{\mathrm{SN}}=A \dot{\rho}_{\mathrm{s}, \mathrm{fb}}$,

where $\dot{\rho}_{\mathrm{s}, \mathrm{fb}}$ is defined by equation (43). Following the analytical model of McKee \& Ostriker (1977) for SN blast waves, the evaporation efficiency parameter $A$ is given by

$A=A_{0}\left(\frac{\rho_{\mathrm{w}}}{\rho_{\mathrm{w}, 0}}\right)^{-4 / 5}\left(\frac{l_{\mathrm{c}}}{l_{\mathrm{c}, 0}}\right)^{-6 / 5}\left(\frac{V_{\mathrm{c}}}{V_{\mathrm{c}, 0}}\right)^{3 / 5}$,

where $\rho_{\mathrm{w}}=\rho-\rho_{\mathrm{c}}$, and the length-scale $l_{\mathrm{c}}$ and the volume $V_{\mathrm{c}}$ of the cold clumps are defined by equations (7) and (9). To express 
1846

\section{H. Braun and W. Schmidt}

variables in dimension-free form, we use the following scales:

$$
\begin{aligned}
T_{0} & =T_{\mathrm{TI}}=1.5 \times 10^{4} \mathrm{~K}, \\
u_{0} & =k_{\mathrm{B}} T_{0} / \mu m_{H}(\gamma-1), \\
A_{0} & =u_{\mathrm{SN}} / 2 u_{0}, \\
\rho_{0} & =\mu m_{\mathrm{H}} \times 10.0 \mathrm{~cm}^{-3}, \\
\rho_{\mathrm{c}, 0} & =10^{-3} \rho_{0}, \\
\rho_{\mathrm{w}, 0} & =\rho_{0}-\rho_{\mathrm{c}, 0}, \\
l_{\mathrm{c}, 0} & =\lambda_{\mathrm{J}, \mathrm{c}}\left(u_{\mathrm{c}}, \rho_{\mathrm{c}, \mathrm{pa}, 0}\right) .
\end{aligned}
$$

(iii) The cold phase gains mass from the warm phase via radiative cooling if the gas is thermally unstable:

$$
\left.\frac{\mathrm{d} \rho_{\mathrm{c}}}{\mathrm{d} t}\right|_{\mathrm{cool}}=\frac{\left(1-f_{\mathrm{th}}\right) \rho_{\mathrm{w}} \Lambda_{\mathrm{eff}}}{u_{\mathrm{w}}-u_{\mathrm{c}}},
$$

where the effective cooling rate $\Lambda_{\text {eff }}$ is defined in Section 4.2 , and $f_{\text {th }}$ is the thermal stability indicator that switches on/off terms in the model equations that are related to the thermal instability:

$1-f_{\text {th }}:= \begin{cases}1 & \text { if conditions (a)-(c) are satisfied } \\ 0 & \text { else. }\end{cases}$

The warm neutral gas is treated to be thermally unstable, in the meaning of currently separating into two phases due to cooling, if the following conditions are met.

(a) The net effect of radiative cooling, Lyc radiation, UV background radiation, and turbulent dissipative heating must decrease the thermal energy, i.e. $\Lambda_{\text {eff }}>0$.

(b) The warm gas density $\rho_{\mathrm{w}, \mathrm{pa}}$ must exceed $0.1 \mu m_{\mathrm{H}} \mathrm{cm}^{-3}$, since this is roughly the minimum density for thermal instability according to the equilibrium cooling curve.

(c) Furthermore, the gas must be largely neutral to be thermally unstable; thus, we assume an upper temperature threshold $T_{\mathrm{TI}} \equiv$ $1.5 \times 10^{4} \mathrm{~K}$ for the cooling instability of the warm gas.

(iv) Massive stars of spectral classes $\mathrm{O}$ and $\mathrm{B}$ are strong emitters of radiation in the far-ultraviolet (FUV). In particular, the photons in range of the Lyc deposit a significant amount of energy $x_{\text {Lyc }}$ per photon (see Section 6.3) in the gas, as they are absorbed and then re-emitted as Lyman $\alpha(\operatorname{Ly} \alpha)$ photons. The number of Lyc photons emitted by young, massive stars per unit volume and per unit time, $\dot{N}_{\text {Lyc,loc }}$, is computed from a convolution of the past star formation rate $\dot{\rho}_{\mathrm{s}}$ and the specific emission rate of Lyc photons $\dot{n}_{\text {Lyc }}$ :

$\dot{N}_{\mathrm{Lyc}, \text { loc }}(t)=\int_{0}^{t} \dot{\rho}_{\mathrm{s}}\left(t^{\prime}\right) \dot{n}_{\mathrm{Lyc}}\left(t-t^{\prime}, Z\right) \mathrm{d} t^{\prime}$.

For $\dot{n}_{\text {Lyc }}\left(t-t^{\prime}, Z\right)$, we use an analytic fit to data from evolutionary synthesis models of a simple star population (Kotulla et al. 2009). Some fraction $f_{\text {leak }}$ of these photons may leak into the environment of the reference volume, while $\dot{N}_{\text {Lyc,ext }}$ photons from external sources may get in. The effective number of Lyc photons per unit time and unit volume that actually ionize hydrogen is then given by

$\dot{N}_{\text {Lyc }}=\left(\dot{N}_{\text {Lyc,loc }}+\dot{N}_{\text {Lyc,ext }}\right)\left(1-f_{\text {leak }}\right)$,

where $f_{\text {leak }} \simeq \exp \left(-\sigma_{\mathrm{H}} X \rho l / m_{\mathrm{H}}\right)$ with the ionization cross-section $\sigma_{\mathrm{H}} \simeq 6.3 \times 10^{-18} \mathrm{~cm}^{-2}$. In case of average hydrogen number densities $\rho X / m_{\mathrm{H}} \geq 1 \mathrm{~cm}^{-3}$ and length-scales $l$ of at least a few parsec, $f_{\text {leak }}$ is negligible small. It is likely that the reference volume is surrounded by an environment of comparable density and size, if sources of Lyc radiation are located there, thus $\dot{N}_{\text {Lyc,ext }}$ is negligible, too. Hence, we set $\dot{N}_{\mathrm{Lyc}}=\dot{N}_{\mathrm{Lyc}, \text { loc }}$ and assume that every hydrogen atom has the same chance to absorb a photon. The radiative evaporation rate is given by:

$\left.\frac{\mathrm{d} \rho_{\mathrm{c}}}{\mathrm{d} t}\right|_{\text {heat }}=-\frac{\dot{N}_{\mathrm{Lyc}} x_{\mathrm{Lyc}} \rho_{\mathrm{c}}}{\left(u_{\mathrm{w}}-u_{\mathrm{c}}\right) \rho}$.

(v) Turbulent energy is dissipated into thermal energy at a rate that is given by

$\epsilon=C_{\epsilon} \frac{e_{\mathrm{t}}^{3 / 2}}{l}$,

where $C_{\epsilon}$ is about unity (see Schmidt, Niemeyer \& Hillebrandt 2006; SF11). In our two-phase model, turbulent dissipation heats the gas in the warm phase, but we assume the temperature of the cold gas to be constant. To compensate turbulent dissipation in the cold phase, we simply transfer an equivalent amount of mass from the cold to the warm phase. Since turbulence is assumed to be homogeneous on length-scales smaller than $l$, the amount of energy dissipated in the cold phase is $m_{\mathrm{c}} \epsilon \mathrm{d} t$ over an infinitesimal time interval $\mathrm{d} t$. Setting this equal to the increase of thermal energy $\left(u_{\mathrm{w}}\right.$ $\left.-u_{\mathrm{c}}\right) \mathrm{d} m_{\mathrm{c}}$ if the mass $\mathrm{d} m_{\mathrm{c}}$ is transferred to the warm phase and substituting $m_{\mathrm{c}}=\rho_{\mathrm{c}} V$, we obtain

$\left.\frac{\mathrm{d} \rho_{\mathrm{c}}}{\mathrm{d} t}\right|_{\text {diss }}=-\frac{C_{\epsilon} e_{\mathrm{t}}^{3 / 2} \rho_{\mathrm{c}}}{l\left(u_{\mathrm{w}}-u_{\mathrm{c}}\right)}$.

(vi) If the cold gas forms small compact clumps embedded in the warm phase, i.e. $l_{\mathrm{c}} \ll l$, we can model interactions between the clumps as collisions. Since collisions cause a certain mass-loss of the cold phase by turbulent mixing and heating, we write

$\left.\frac{\mathrm{d} \rho_{\mathrm{c}}}{\mathrm{d} t}\right|_{\text {coll }}=-\epsilon_{\mathrm{cc}} \rho_{\mathrm{c}, \mathrm{pa}} r_{\mathrm{c}, \mathrm{coll}} l_{\mathrm{c}}^{3}$.

The effect of clump collisions on the cold gas density is modelled by the efficiency parameter $\epsilon_{\mathrm{cc}}$ and the collision rate

$r_{\mathrm{c}, \mathrm{coll}}=n_{\mathrm{c}} V \frac{v_{\mathrm{c}, \mathrm{coll}}}{l_{\mathrm{c}, \text { free }}}$.

Setting the average volume of a cold clump equal to $\pi l_{\mathrm{c}}^{3} / 6$, the number density of the clumps is $n_{\mathrm{c}} \sim\left(6 V_{\mathrm{c}} / \pi l_{\mathrm{c}}^{3}\right) / V$ and the meanfree path $l_{\mathrm{c} \text {,free }}=\left(\pi l_{\mathrm{c}}^{2} n_{\mathrm{c}}\right)^{-1}$. The rms velocity of the clump motion in the surrounding warm medium can be estimated from the square root of the turbulent energy $e_{\mathrm{t}}$, corrected by the internal velocity dispersion $\sigma_{\mathrm{c}}^{2}$ of the clumps (see equation 12):

$v_{\mathrm{c}, \mathrm{coll}}=\left(2 e_{\mathrm{t}}-3 \sigma_{\mathrm{c}}^{2}\right)^{1 / 2}=\left[2 e_{\mathrm{t}}\left(1-\left(\frac{l_{\mathrm{c}}}{l}\right)^{2 \eta}\right)\right]^{1 / 2}$,

With the above definitions, it follows that

$r_{\mathrm{c}, \text { coll }}=\frac{36 V_{c}^{2}}{\pi l_{\mathrm{c}}^{4} V}\left[2 e_{\mathrm{t}}\left(1-\left(\frac{l_{\mathrm{c}}}{l}\right)^{2 \eta}\right)\right]^{1 / 2}$,

where $V_{c}$ and $l_{\mathrm{c}}$ are given by equations (9) and (7), respectively.

Collecting all six contributions, the evolutionary equation of the cold-phase density can be written as

$$
\begin{aligned}
\dot{\rho}_{\mathrm{c}}= & -\dot{\rho}_{\mathrm{s}}-A \dot{\rho}_{\mathrm{s}, \mathrm{fb}}-\frac{1}{u_{\mathrm{w}}-u_{\mathrm{c}}} \\
& \times\left[-\left(1-f_{\mathrm{th}}\right) \rho_{\mathrm{w}} \Lambda_{\mathrm{eff}}+\frac{\dot{N}_{\mathrm{Lyc}} x_{\mathrm{Lyc}} \rho_{\mathrm{c}}}{\rho}+\frac{C_{\epsilon} e_{\mathrm{t}}^{3 / 2} \rho_{\mathrm{c}}}{l}\right] \\
& -\frac{36 \epsilon_{\mathrm{cc}} \rho_{\mathrm{c}, \mathrm{pa}} V_{c}^{2}}{\pi l_{\mathrm{c}}}\left[2 e_{\mathrm{t}}\left(1-\left(\frac{l_{\mathrm{c}}}{l}\right)^{2 \eta}\right)\right]^{1 / 2},
\end{aligned}
$$

(C) 2012 The Authors, MNRAS 421, 1838-1860 Monthly Notices of the Royal Astronomical Society (c) 2012 RAS 
A model of the turbulent multi-phase ISM

and the change of the gas density in the warm phase follows from mass conservation $\left(\dot{\rho}_{\mathrm{w}}+\dot{\rho}_{\mathrm{c}}+\dot{\rho}_{\mathrm{s}, \text { eff }}=0\right)$ :

$\dot{\rho}_{\mathrm{w}}=-\dot{\rho}_{\mathrm{c}}-\dot{\rho}_{\mathrm{s}, \mathrm{eff}}$.

\subsection{Exchange of energy between the phases}

In numerical simulations of thermally bistable turbulence (e.g. Audit \& Hennebelle 2010; Seifried et al. 2011), most of the gas in the cold phase is close to the isothermal equilibrium branch of the cooling curve. This is mainly caused by the higher opacity of the dense, cold gas that lowers the efficiency of radiative cooling. Other processes that affect the gas temperature in the cold phase, such as the gravitational collapse of dense regions or chemical reactions, are not explicitly treated in our model. Consequently, the specific thermal energy of the cold phase $u_{\mathrm{c}}$ is assumed to be constant. We set the temperature to a fiducial value $T_{\mathrm{c}}=50 \mathrm{~K}$, corresponding to the lower cut-off of the cooling curve in galaxy simulations.

The specific thermal energy of the warm phase, on the other hand, is changed by the processes that are discussed in Section 4.1. The effects of these processes on $u_{\mathrm{w}}$ are as follows (see also Fig. 2).

(i) $\mathrm{SNe}$ heat the warm gas and transfer gas from the cold to the warm phase via evaporation. The energy release per unit mass is $u_{\mathrm{SN}} \approx 6 \times 10^{49} \mathrm{erg}_{\odot}^{-1}$ (see Section 6.3). Assuming that a certain fraction $\epsilon_{\mathrm{SN}} u_{\mathrm{SN}}$ of the feedback is non-thermal (see Section 4.3), we have

$\left.\frac{\mathrm{d}\left(\rho_{\mathrm{w}} u_{\mathrm{w}}\right)}{\mathrm{d} t}\right|_{\mathrm{SN}}=\left[\left(1-\epsilon_{\mathrm{SN}}\right) u_{\mathrm{SN}}+A u_{\mathrm{c}}\right] \dot{\rho}_{\mathrm{s}, \mathrm{fb}}$.

The efficiency parameter $A$ is defined by equation (49). The rate of change of the warm gas density due to $\mathrm{SN}$ feedback is given by the mass ejection from $\mathrm{SNe}$ and the evaporation of cold gas:

$\left.\frac{\mathrm{d} \rho_{\mathrm{w}}}{\mathrm{d} t}\right|_{\mathrm{SN}}=(1+A) \dot{\rho}_{\mathrm{s}, \mathrm{fb}}$,

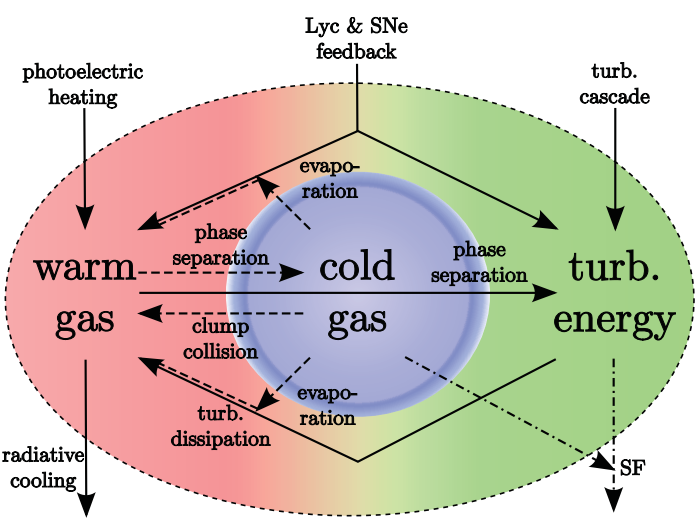

Figure 2. Scheme of the exchange of energy. The ellipse depicts the energy content of the gas, which is separated into three budgets: the thermal energy of the warm gas $\rho_{\mathrm{w}} u_{\mathrm{w}}$ (red) and the cold gas $\rho_{\mathrm{c}} u_{\mathrm{c}}$ (blue) and the non-thermal, turbulent energy $\rho e_{\mathrm{t}}$ (green). Cold-phase thermal energy can only change by loss or gain of mass, as $u_{\mathrm{c}}=$ const., which is shown by dashed arrows, while processes not intermixing cold gas with any phase have solid arrows. Star formation removes mass from the cold gas, along with its energies, which is shown in dot-dashed arrows.
Combining the above equations, it follows that

$\left.\left.\frac{\mathrm{d} u_{\mathrm{w}}}{\mathrm{d} t}\right|_{\mathrm{SN}}=\left[\left(1-\epsilon_{\mathrm{SN}}\right) u_{\mathrm{SN}}+A u_{\mathrm{c}}-(1+A) u_{\mathrm{w}}\right)\right] \frac{\dot{\rho}_{\mathrm{s}, \mathrm{fb}}}{\rho_{\mathrm{w}}}$.

(ii) If the warm phase is thermally stable, the warm gas cools (or heats) at a rate given by its effective cooling function $\Lambda_{\text {eff }}$. Once the thermal instability sets in, gas in the warm phase is converted into cold gas without changing the temperature of the remaining warm gas. We also assume that the cooling instability produces turbulent energy with an efficiency $\epsilon_{\mathrm{tt}}$ relative to the cooling function. Consequently, the total change of the internal energy density of the warm phase can be written as

$$
\begin{aligned}
\left.\frac{\mathrm{d}\left(\rho_{\mathrm{w}} u_{\mathrm{w}}\right)}{\mathrm{d} t}\right|_{\text {cool }}= & -\rho_{\mathrm{w}} \Lambda_{\mathrm{eff}}+\left.u_{\mathrm{c}} \frac{\mathrm{d} \rho_{\mathrm{w}}}{\mathrm{d} t}\right|_{\text {cool }} \\
& -\left(1-f_{\mathrm{th}}\right) \epsilon_{\mathrm{tt}} \rho_{\mathrm{w}} \Lambda_{\mathrm{eff}} .
\end{aligned}
$$

Since the rate of change of the warm gas density due to cooling is given by equation (50) multiplied by minus one, we obtain

$\left.\frac{\mathrm{d} u_{\mathrm{w}}}{\mathrm{d} t}\right|_{\text {cool }}=-f_{\mathrm{th}} \Lambda_{\mathrm{eff}}-\left(1-f_{\mathrm{th}}\right) \epsilon_{\mathrm{tt}} \Lambda_{\mathrm{eff}}$.

The effective cooling rate $\Lambda_{\text {eff }}$ is defined by

$\Lambda_{\text {eff }}=\Lambda_{\text {rad }}-\Gamma_{\text {PAH }}-\Gamma_{\text {Lyc }}-\epsilon$,

where $\Lambda_{\text {rad }}$ is the specific radiative cooling rate. In this model, we use a tabled atomic cooling function, computed using the photo-ionization package CLOUDY (version 08.00), last described by Ferland et al. (1998), without considering molecules or dust. $\Gamma_{\mathrm{PAH}}$ is the photo-electric heating rate (Wolfire et al. 1995) due to the external radiation field $I_{v}$ modified by a factor of $Z / Z_{\odot}$, and $\epsilon$ is the turbulent dissipation rate per unit mass (equation 55). The volume rate of heating by Lyc photons is given by

$\left.\frac{\mathrm{d}\left(\rho_{\mathrm{w}} u_{\mathrm{w}}\right)}{\mathrm{d} t}\right|_{\text {heat }}=\dot{N}_{\text {Lyc }} x_{\text {Lyc }}+\left.u_{\mathrm{c}} \frac{\mathrm{d} \rho_{\mathrm{w}}}{\mathrm{d} t}\right|_{\text {heat }}$.

Hence, the specific heating rate is

$\Gamma_{\text {Lyc }}=\left.\frac{\mathrm{d} u_{\mathrm{w}}}{\mathrm{d} t}\right|_{\text {heat }}=\frac{\dot{N}_{\mathrm{Lyc}} x_{\mathrm{Lyc}}}{\rho}$.

(iii) Since cold gas is transferred to the warm phase by clump collisions, we have

$\left.\frac{\mathrm{d}\left(\rho_{\mathrm{w}} u_{\mathrm{w}}\right)}{\mathrm{d} t}\right|_{\text {coll }}=\left.u_{\mathrm{c}} \frac{\mathrm{d} \rho_{\mathrm{c}}}{\mathrm{d} t}\right|_{\text {coll }}$.

The corresponding rate of change of the specific energy is given by (see equation (57))

$\left.\frac{\mathrm{d} u_{\mathrm{w}}}{\mathrm{d} t}\right|_{\text {coll }}=-\epsilon_{\mathrm{cc}}\left(u_{\mathrm{w}}-u_{\mathrm{c}}\right) \frac{\rho_{\mathrm{c}, \mathrm{pa}} r_{\mathrm{c}, \mathrm{coll}} l_{\mathrm{c}}^{3}}{\rho_{\mathrm{w}}}$,

where $\epsilon_{\mathrm{cc}}$ is the efficiency parameter of the collisions, and the collision rate $r_{\mathrm{c}, \text { coll }}$ is defined by equation (60).

Adding up the contributions (i)-(v), the dynamical equation for the thermal energy of the warm phase becomes

$$
\begin{aligned}
\dot{u}_{\mathrm{w}}= & {\left.\left[\left(1-\epsilon_{\mathrm{SN}}\right) u_{\mathrm{SN}}+A u_{\mathrm{c}}-(1+A) u_{\mathrm{w}}\right)\right] \frac{\dot{\rho}_{\mathrm{s}, \mathrm{fb}}}{\rho_{\mathrm{w}}} } \\
& -\left[f_{\mathrm{th}}+\left(1-f_{\mathrm{th}}\right) \epsilon_{\mathrm{tt}}\right] \Lambda_{\mathrm{eff}} \\
& -\epsilon_{\mathrm{cc}}\left(u_{\mathrm{w}}-u_{\mathrm{c}}\right) \frac{\rho_{\mathrm{c}, \mathrm{pa}} r_{\mathrm{c}, \mathrm{coll}} l_{\mathrm{c}}^{3}}{\rho_{\mathrm{w}}} .
\end{aligned}
$$




\subsection{Turbulent energy production and dissipation}

To formulate an equation for the turbulent energy, we assume that energy is injected at constant rate $\Sigma$ by large-scale forcing. The rate of energy injection determines the flux of kinetic energy that is transported through the turbulent cascade from larger to smaller scales. For purely hydrodynamic isotropic turbulence, the energy flux is independent of the length-scale and equal to the dissipation rate $\epsilon$ in statistical equilibrium. Applying the method of (adaptively refined) LES, $\Sigma$ can be computed from the SF11 closure for the compressible turbulent energy cascade. For the one-zone formulation of our model, we simply express $\Sigma$ in terms of the typical magnitude of the turbulent velocity fluctuations $\mathcal{V}$ induced by the turbulent cascade on the length-scale $l$ :

$\Sigma=C_{\epsilon} \rho \frac{\mathcal{V}^{3 / 2}}{l}$.

For pure hydrodynamical turbulence, $\Sigma=\epsilon$ and $e_{\mathrm{t}}=0.5 \mathcal{V}^{2}$ in equilibrium.

Neglecting turbulent diffusion and collecting the terms that exchange energy between the gas phases and turbulence, the following rate equation for the turbulent energy results:

$$
\begin{aligned}
\dot{e}_{\mathrm{t}}= & \left(\epsilon_{\mathrm{SN}} u_{\mathrm{SN}}-e_{\mathrm{t}}\right) \frac{\dot{\rho}_{\mathrm{s}, \mathrm{fb}}}{\rho}+\left(1-f_{\mathrm{th}}\right) \epsilon_{\mathrm{tt}} \Lambda_{\mathrm{eff}} \frac{\rho_{\mathrm{w}}}{\rho} \\
& +\frac{\Sigma}{\rho}-C_{\epsilon} \frac{e_{\mathrm{t}}^{3 / 2}}{l} .
\end{aligned}
$$

The three sources of turbulent energy production are SN feedback on length-scales comparable to $l$, the cooling instability and the turbulent energy cascade. The two efficiency parameters $\epsilon_{\mathrm{SN}}$ and $\epsilon_{\mathrm{tt}}$ determine the coupling of the unresolved processes to the turbulent energy. In numerical simulations, in which $l$ corresponds to the grid scale, these parameters have to be chosen appropriately. The crucial problem is that, in contrast to the cascade of turbulent eddies in the inertial sub-range of isotropic turbulence, $\mathrm{SN}$ feedback and the cooling instability are not self-similar. We can only assume that particular efficiency parameters apply to certain ranges of scales. One option is to use small-scale simulations of the interaction of $\mathrm{SNe}$ blast waves with the ISM and thermally bistable flows in periodic boxes to estimate these parameters. On the other hand, the model can be calibrated a posteriori in large-scale simulations such that observational constraints are met. We also show in Section 6 that one-zone calculations can be utilized to find reasonable choices of the efficiency parameters.

Combining equations (61), (62), (73), (75) and $u_{\mathrm{c}}=$ const., the following law of energy conservation equation is obtained:

$$
\begin{aligned}
\frac{\mathrm{d}\left(\rho\left(u+e_{\mathrm{t}}\right)\right)}{\mathrm{d} t}= & -\dot{\rho}_{\mathrm{s}}\left(u_{\mathrm{c}}+e_{\mathrm{t}}\right)+\dot{\rho}_{\mathrm{s}, \mathrm{fb}} u_{\mathrm{SN}} \\
& -\rho_{\mathrm{w}} \Lambda_{\mathrm{rad}}+\rho_{\mathrm{w}} \Gamma_{\mathrm{PAH}} \\
& +\dot{N}_{\mathrm{Lyc}} x_{\mathrm{Lyc}}+\Sigma .
\end{aligned}
$$

\section{LIMITING CASES}

\subsection{One-phase medium}

If most of the gas cools down to temperatures close to $T_{\mathrm{c}}$, the separation between a dynamic warm phase and a star-forming cold phase breaks down. Assuming that the warm gas can also form stars if $u_{\mathrm{w}} \leq u_{\mathrm{w}, \min }$, where $u_{\mathrm{w}, \min } \geq u_{\mathrm{c}}$, the star formation law (15) becomes

$\dot{\rho}_{\mathrm{s}}=\frac{\rho}{t_{\mathrm{s}}}$,

where $\rho_{\mathrm{c}, \mathrm{pa}}=\rho$ and $\dot{\rho}=-\dot{\rho}_{\mathrm{s} \text {, eff }}$. In this article, our focus is on a statistical description. By choosing a length-scale $l \gg l_{\mathrm{c}}=\lambda_{\mathrm{J}}(\rho)$, the star formation time-scale $t_{\mathrm{s}}$ can be calculated as in Section 3. When applying this model as an SGS model in numerical simulations, however, the Truelove criterion requires $l \leq l_{\mathrm{J}}(\rho) / 4$. Consequently, an alternative parametrization of the star formation time-scale has to be applied in the one-phase limit. This is left for future work. Practically, this case will occur in simulations of individual galaxies with high resolution, but usually not in cosmological simulations, where cold-gas clumps are sufficiently below the resolution limit.

Since the cooling instability vanishes, and the exchange of energy between the phases as well as the collisions of cold clumps become meaningless, the equation for the specific thermal energy becomes

$\left.\dot{u}_{\mathrm{w}} \simeq\left[\left(1-\epsilon_{\mathrm{SN}}\right) u_{\mathrm{SN}}-u_{\mathrm{c}}\right)\right] \frac{\dot{\rho}_{\mathrm{s}, \mathrm{fb}}}{\rho}-\Lambda_{\mathrm{eff}}$.

The subtraction of $u_{\mathrm{c}}$ in the factor that is multiplied with the feedback rate results from the removal of the energy in the gas that forms stars from the gas energy budget (see equation 76). Practically, we can neglect the difference because $u_{\mathrm{SN}} \gg u_{\mathrm{c}}$. The thermal energy equation is complemented by the simplified turbulent energy equation:

$\dot{e}_{\mathrm{t}}=\frac{1}{\rho}\left(\epsilon_{\mathrm{SN}} u_{\mathrm{SN}} \dot{\rho}_{\mathrm{s}, \mathrm{fb}}+\Sigma\right)-C_{\epsilon} \frac{e_{\mathrm{t}}^{3 / 2}}{l}$.

It is instructive to consider the asymptotic limit $u_{\mathrm{w}} \simeq u_{\mathrm{c}}=$ const. Then we can set $\dot{u}_{\mathrm{w}} \simeq 0$. For net heating $\left(\Lambda_{\text {eff }}<0\right)$, this equation cannot be fulfilled because both terms are positive. If $\Lambda_{\text {eff }}>0$, on the other hand, the feedback rate is approximately given by a balance between thermal heating by $\mathrm{SNe}$ and turbulence production by cooling:

$\dot{\rho}_{\mathrm{s}, \mathrm{fb}} \simeq \frac{\rho \Lambda_{\mathrm{eff}}}{\left(1-\epsilon_{\mathrm{SN}}\right) u_{\mathrm{SN}}}$.

Since $\dot{\rho}_{\mathrm{s}, \mathrm{fb}}$ is related to the star formation rate (77) via equation (43), the above equation imposes a condition on the effective cooling rate so that $u_{\mathrm{w}} \simeq u_{\mathrm{c}}=$ const.

\subsection{Equilibrium solutions}

Of particular interest is the case of self-regulation, for which the star formation rate is low and nearly constant: $\dot{\rho}_{\mathrm{s}} \simeq \dot{\rho}_{\mathrm{s} \text { eq }}=$ const. A low star formation rate means that changes in the gas density are negligible in first-order approximation. In addition, we assume that the temperature of the warm phase and the specific turbulent energy are approximately constant in the self-regulated regime and that the cooling instability is active $(f=0)$. For simplicity, we neglect clump collisions. Setting $\dot{u}_{\mathrm{w}} \simeq 0$ and $\dot{e}_{\mathrm{t}} \simeq 0$ in equations (73) and (75), respectively, it follows that

$\left.\left[\left(1-\epsilon_{\mathrm{SN}}\right) u_{\mathrm{SN}}+A u_{\mathrm{c}}-(1+A) u_{\mathrm{w}, \mathrm{eq}}\right)\right] \frac{\dot{\rho}_{\mathrm{s}, \mathrm{fb}}}{\rho_{\mathrm{w}}}-\epsilon_{\mathrm{tt}} \Lambda_{\mathrm{eff}} \simeq 0$,

$\left(\epsilon_{\mathrm{SN}} u_{\mathrm{SN}}-e_{\mathrm{t}, \mathrm{eq}}\right) \frac{\dot{\rho}_{\mathrm{s}, \mathrm{fb}}}{\rho}+\epsilon_{\mathrm{tt}} \Lambda_{\mathrm{eff}} \frac{\rho_{\mathrm{w}}}{\rho}+\frac{\Sigma}{\rho}-C_{\epsilon} \frac{e_{\mathrm{t}, \mathrm{eq}}^{3 / 2}}{l} \simeq 0$,

where $u_{\mathrm{w}, \mathrm{eq}}$ and $e_{\mathrm{t}, \mathrm{eq}}$ are the equilibrium values.

(C) 2012 The Authors, MNRAS 421, 1838-1860 Monthly Notices of the Royal Astronomical Society (C) 2012 RAS 
Equation (82) imposes a condition on the feedback rate. By substituting the effective cooling rate (68) for $\Lambda_{\text {eff }}$, we obtain

$$
\begin{aligned}
\left(\epsilon_{\mathrm{SN}} u_{\mathrm{SN}}-e_{\mathrm{t}, \mathrm{eq}}\right) \frac{\dot{\rho}_{\mathrm{s}, \mathrm{fb}}}{\rho} \simeq & C_{\epsilon}\left(\rho+\epsilon_{\mathrm{tt}} \rho_{\mathrm{w}}\right) \frac{e_{\mathrm{t}, \mathrm{eq}}^{3 / 2}}{l}-\Sigma \\
& +\epsilon_{\mathrm{tt}} \rho_{\mathrm{w}}\left(\Gamma_{\mathrm{PAH}}+\Gamma_{\mathrm{Lyc}}-\Lambda_{\mathrm{rad}}\right) .
\end{aligned}
$$

For any reasonable choice of parameters, $e_{\mathrm{t} \text {,eq }} \ll \epsilon_{\mathrm{SN}} u_{\mathrm{SN}}$. Consequently, a solution exits only if

$C_{\epsilon}\left(\rho+\epsilon_{\mathrm{tt}} \rho_{\mathrm{w}}\right) \frac{e_{\mathrm{t}, \mathrm{eq}}^{3 / 2}}{l} \geq \Sigma+\epsilon_{\mathrm{tt}} \rho_{\mathrm{w}}\left(\Lambda_{\mathrm{rad}}-\Gamma_{\mathrm{PAH}}-\Gamma_{\mathrm{Lyc}}\right)$.

The right-hand side is always positive if the cooling instability is active, because $\Lambda_{\text {rad }}-\Gamma_{\text {PAH }}-\Gamma_{\text {Lyc }} \geq \Lambda_{\text {eff }}>0$. For this reason, the above constraint implies that there must be a minimal turbulent energy for self-regulation:

$\min e_{\mathrm{t}, \mathrm{eq}}=\left(\frac{\left[\Sigma+\epsilon_{\mathrm{tt}} \rho_{\mathrm{w}}\left(\Lambda_{\mathrm{rad}}-\Gamma_{\mathrm{PAH}}-\Gamma_{\mathrm{Lyc}}\right)\right] l}{C_{\epsilon}\left(\rho+\epsilon_{\mathrm{tt}} \rho_{\mathrm{w}}\right)}\right)^{2 / 3}$.

This an important implication of the multi-phase model.

The stellar feedback rate is also a constant in the self-regulated regime. This follows immediately from equation (43) for a constant star formation rate. Then

$\dot{\rho}_{\mathrm{s}, \mathrm{fb}} \simeq \beta \dot{\rho}_{\mathrm{s}, \mathrm{eq}}$,

where

$\beta=\int_{t_{\mathrm{b}}}^{t_{\mathrm{e}}} \frac{1}{M_{*}} \frac{\mathrm{d} N_{*}}{\mathrm{~d} m_{*}} \frac{\mathrm{d} m_{*}}{\mathrm{~d} t^{\prime}} \mathrm{d} t^{\prime}$

In principle, one could invert the equations for a given star formation rate, $\dot{\rho}_{\mathrm{s}, \text { eq }}$, and the effective pressure equilibrium between the phases, to obtain the cold and warm-gas densities. Due to the high non-linearity of these equations, particularly the molecular hydrogen fraction, this is very difficult in practice. It is easier to search for equilibrium solutions by computing the full set of rate equations and identifying solutions that are close to equilibrium values satisfying equations (81), (82) and (84) in certain time intervals.

By substituting equation (82) into equation (81), the following expression for the equilibrium energy of the warm phase can be obtained:

$$
\begin{aligned}
u_{\mathrm{w}, \mathrm{eq}} \simeq & \frac{u_{\mathrm{SN}}}{1+A}+\frac{A}{1+A} u_{\mathrm{c}}-\frac{1}{1+A} e_{\mathrm{t}, \mathrm{eq}} \\
& -\frac{1}{(1+A) \beta \dot{\rho}_{\mathrm{s}, \mathrm{eq}}}\left(C_{\epsilon} \rho \frac{e_{\mathrm{t}, \mathrm{eq}}^{3 / 2}}{l}-\Sigma\right) .
\end{aligned}
$$

For $\Sigma=0$ (no turbulence feeding by instabilities on lengthscales greater than $l$ ) and $e_{\mathrm{t} \text { eq }}=0$ (turbulent energy is neglected), the $\mathrm{SH} 03$ equilibrium solution for $u_{\mathrm{w} \text {,eq }}$ results, with the exception of the factor $A /(1+A)$. Since $A \gg 1$, however, this factor is very close to unity and the result is practically the same. In our model, the cooling instability produces turbulent energy on top of the turbulent cascade. Thus, $e_{\mathrm{t} \text {.eq }}>\left(\Sigma l / C_{\epsilon} \rho\right)^{2 / 3}$ (see equation 81$)$ and

$u_{\mathrm{w}, \mathrm{eq}}<u_{\mathrm{SH}}:=\frac{u_{\mathrm{SN}}}{1+A}+u_{\mathrm{c}}$.

As a consequence, we expect that the temperature of warm gas close to equilibrium decreases in numerical simulations with a turbulence SGS model, because a fraction of the energy is in nonthermal form.

(c) 2012 The Authors, MNRAS 421, 1838-1860

Monthly Notices of the Royal Astronomical Society (C) 2012 RAS
A model of the turbulent multi-phase ISM

\section{MODELLING THE EVOLUTION} OF A SINGLE ZONE

The set of six coupled non-linear differential equations $(42,61,62$, $73,75,46)$, as defined in Section 4 , describe how the gas in the reference volume evolves with time. By numerically integrating the model equations over closed boxes (i.e. single zones), we obtain statistical models for a wide range of initial conditions and parameters. These models also allow us to find equilibrium states, for which the star formation rate is small and nearly constant (see Section 5.2). To characterize the star formation rate, we define a dimensionless star formation efficiency by

$\varepsilon_{\mathrm{ff}}=\frac{\dot{\rho}_{\mathrm{s}} t_{\mathrm{ff}}}{\rho}$.

This is the fraction of the total gas mass in the reference volume that is turned into stars over a free-fall time-scale $t_{\mathrm{ff}}=\sqrt{3 \pi / 32 G \rho}$. It is to be distinguished from $\mathrm{SFR}_{\mathrm{c}, \mathrm{ff}}$, which is sometimes also called star formation efficiency. However, $\mathrm{SFR}_{\mathrm{c}, \mathrm{ff}}$ specifies the fraction of cold molecular gas converted per free-fall time (see Section 3 ).

If not stated otherwise, the following standard model parameters are used:

$\eta=1 / 3, \quad b=2 / 3$,

$\epsilon_{\mathrm{tt}}=0.025, \quad \epsilon_{\mathrm{SN}}=0.085$

$\epsilon_{\mathrm{cc}}=0.0, \quad x_{\mathrm{Lyc}}=0.1 \mathrm{eV}$

$l=15 \mathrm{pc}, \quad f_{\text {loss }}=0.6$

We comment on the choice of these units in Section 6.3. Furthermore, metal enrichment is turned off by setting $\zeta_{\mathrm{m}}=0$. We specify the strength of the turbulent energy injection $\Sigma$ by relating the velocity scale $V$ (see equation 74 ) to the speed of sound at the temperature $T_{\mathrm{TI}}=1.5 \times 10^{4} \mathrm{~K}$, corresponding to the maximum thermal energy $u_{\mathrm{TI}}$ of thermally unstable gas. This results in the Mach number

$\mathcal{M}_{\Sigma}=\frac{V}{c_{\mathrm{TI}}}=\left(\frac{2}{\gamma(\gamma-1) u_{\mathrm{TI}}}\right)^{1 / 2}\left(\frac{\Sigma l}{C_{\epsilon} \rho}\right)^{1 / 3}$

as a basic parameter for the external (large-scale) energy injection relative to the maximal turbulence production by the thermal instability. We use the standard value $\mathcal{M}_{\Sigma}=0.2$.

In Sections 6.1 and 6.2, sample evolutions of gas are discussed. The gas, as well as the formed stars, in these evolutions is confined to the reference volume, i.e. nothing but energy enters or leaves the volume. If the reference volume was embedded in a more realistic, inhomogeneous, dynamic environment, as in an isolated disc galaxy simulation, it would exchange mass with its environment, as gas pressure is subject to strong variation, depending on the environment this may lead to substantial inflows or outflows, and stars may leave the area due to their drift. This is beyond the scope of a single-zone model, but to demonstrate the effects of basic model parameters it is still useful. For the equilibrium solutions discussed in Section 6.3, however, the latter is no objection.

\subsection{Feedback sequence}

As described in Sections 4.1 and 4.2, stellar feedback is determined by the evolution of the stellar populations within the reference volume (e.g. SN feedback starts when the first SNe II light up). As an example, we consider the evolution of gas with number density $n=\rho /\left(\mu m_{\mathrm{H}}\right)=75 \mathrm{~cm}^{-3}$, solar metallicity and no turbulent energy injection (i.e. $\Sigma=0$ ). To single out the effects of the stellar feedback, we artificially suppress star formation after 1 Myr has passed. The results are shown in Fig. 3. The evolution starts in an 


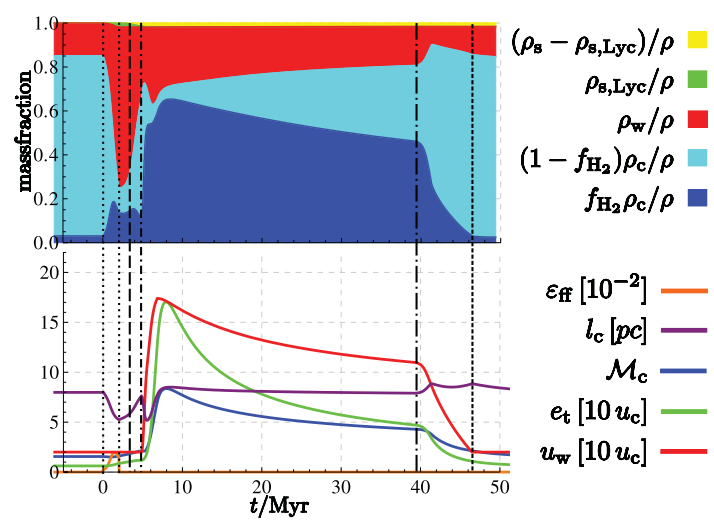

Figure 3. Demonstration of the feedback sequence. Star formation is only active for $t \in[0, \ldots, 1] \mathrm{Myr}$ (period between the dotted vertical lines) and set to zero at later time. The upper panel shows the mass fractions of the different phases as area fillings [stellar fraction $\rho_{\mathrm{s}} / \rho$, contribution of the young Lyc-emitting population $\rho_{\mathrm{s}, \mathrm{Lyc}} / \rho$, warm fraction $\rho_{\mathrm{w}} / \rho$, neutral cold fraction $\rho_{\mathrm{c}}\left(1-f_{\mathrm{c}, \mathrm{H}_{2}}\right) / \rho$ and molecular fraction $\left.f_{\mathrm{c}, \mathrm{H}_{2}} \rho_{\mathrm{c}} / \rho\right]$. In the lower panel, the evolution of the star formation efficiency $\varepsilon_{\mathrm{ff}}$, the rms Mach number of motions in the cold phase $\mathcal{M}_{\mathrm{c}}$, the size of cold clumps $l_{\mathrm{c}}$, the thermal energy of the warm phase $u_{\mathrm{w}}$ and the turbulent energy $e_{\mathrm{t}}$ are plotted.

equilibrium between heating and cooling without star formation. Then star formation is activated within the interval indicated by the vertical dotted lines. Lyc heating by the newly formed stars quickly lowers the star formation efficiency $\varepsilon_{\mathrm{ff}}$ defined by equation (88), as the cold-phase gas fraction decreases, $l_{\mathrm{c}}$ drops while $f_{\mathrm{c}, \mathrm{H}_{2}}$ grows, and the warm-gas pressure rises. But the specific energy $u_{\mathrm{w}}$ remains low because radiative cooling dominates in the warm phase. When Lyc heating begins to decrease (vertical long-dashed line), more warm gas can cool down and $f_{\mathrm{c}, \mathrm{H}_{2}}$ drops due to the shift in the pressure equilibrium between the phases. After the start of $\mathrm{SN}$ feedback (short dot-dashed vertical line) $u_{\mathrm{w}}$ and $\rho_{\mathrm{w}}$ rise quickly, and the ensuing compression of the cold phase results in an increase of $f_{\mathrm{c}, \mathrm{H}_{2}}$. The kink in the evolution of $l_{\mathrm{c}}$ and $\mathcal{M}_{\mathrm{c}}$ that can be seen shortly after the onset of feedback is caused by the production of turbulent energy, which is slightly lagging behind. As SN feedback becomes weaker, $u_{\mathrm{w}}, e_{\mathrm{t}}$ and $f_{\mathrm{c}, \mathrm{H}_{2}}$ decline. Once all $\mathrm{SN}$ progenitors

are gone, SN feedback ceases (long dot-dashed vertical line). Then the warm gas cools down to the initial temperature (reaching it at the short dashed line), and all quantities and gas fractions evolve back to the values for the equilibrium of heating and cooling without star formation.

\subsection{Dynamical evolution}

The main parameters of our model are the initial total gas density $n$, the metallicity $Z$ and the forcing Mach number $\mathcal{M}_{\Sigma}$, corresponding to the turbulent energy injection rate $\Sigma$. In this section, we describe the dependence of the phase evolution and star formation history on these parameters. In addition to $\rho, Z$ and $\mathcal{M}_{\Sigma}$, several coefficients determine the relative contributions from unresolved processes. The influence of these coefficients is discussed in Section 6.3 .

Three sample evolutions are plotted in Fig. 4 for the initial number densities $n=35,50$ and $75 \mathrm{~cm}^{-3}, Z=\mathrm{Z}_{\odot}$ and $\mathcal{M}_{\Sigma}=0.2$. For the lowest density, no star formation occurs at all, except for a negligible fraction in the very beginning (the spurious SN feedback produced by these stars causes the kink in the cooling curve of $u_{\mathrm{w}}$ at $t \simeq$ 4.2 Myr). The final $\rho_{\mathrm{c}} / \rho_{\mathrm{w}}$ is determined by the equilibrium between turbulent dissipation, photoelectric heating and radiative cooling. The final $e_{\mathrm{t}}$ is fixed by the equilibrium between the production of turbulent energy by the thermal instability and large-scale injection and turbulent dissipation. For higher values of $n$ (middle and right panels of Fig. 4), on the other hand, a markedly different evolution can be seen. Following an initial transient phase that ends after about $10 \mathrm{Myr}$, a stationary mode of star formation is entered, in which the star formation efficiency $\varepsilon_{\mathrm{ff}}$ is a few per million. During the transient phase, there are three more or less distinct maxima of $\varepsilon_{\mathrm{ff}}$. This behaviour can be understood as follows. The initial rise of $\rho_{\mathrm{w}} / \rho$ due to Lyc heating by the first stars causes a compression of the cold gas. This results in an increasing molecular hydrogen fraction and, thus, an enhancement of $\varepsilon_{\mathrm{ff}}$. Depletion of the cold phase reverses this trend. As Lyc heating fades out, the star formation efficiency increases again, resulting in a second, but weaker peak. Due to the cooling of the warm phase, which exerts pressure on the cold phase, $\rho_{\text {c,pa }}$ is declining and $\varepsilon_{\mathrm{ff}}$ is lowered. The first SNe raise $u_{\mathrm{w}}$ and the subsequent growth of turbulent energy causes the third maximum.

By comparing Figs 4 and 5, where the latter figure shows plots for three one-zone models with an initial density $n=75 \mathrm{~cm}^{-3}$, but
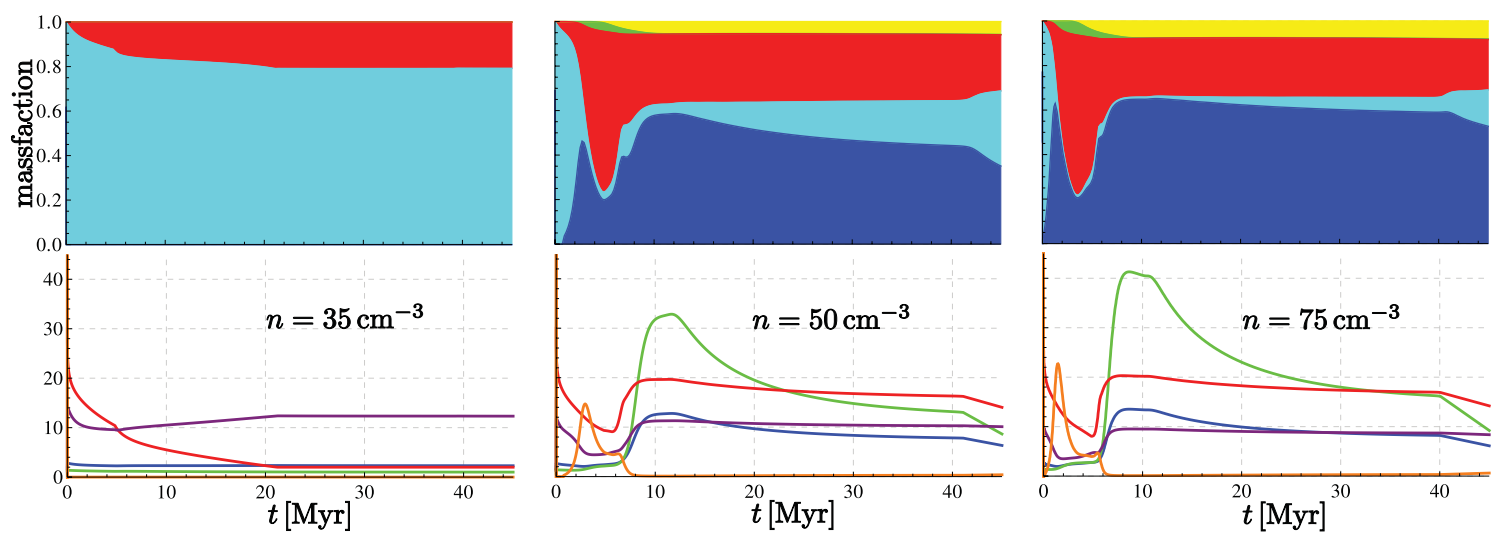

Figure 4. One-zone models for different initial gas densities at solar metallicity and moderate external driving $\mathcal{M}_{\Sigma}=0.2$. See the legend in Fig. 3 for a definition of the plotted quantities. 

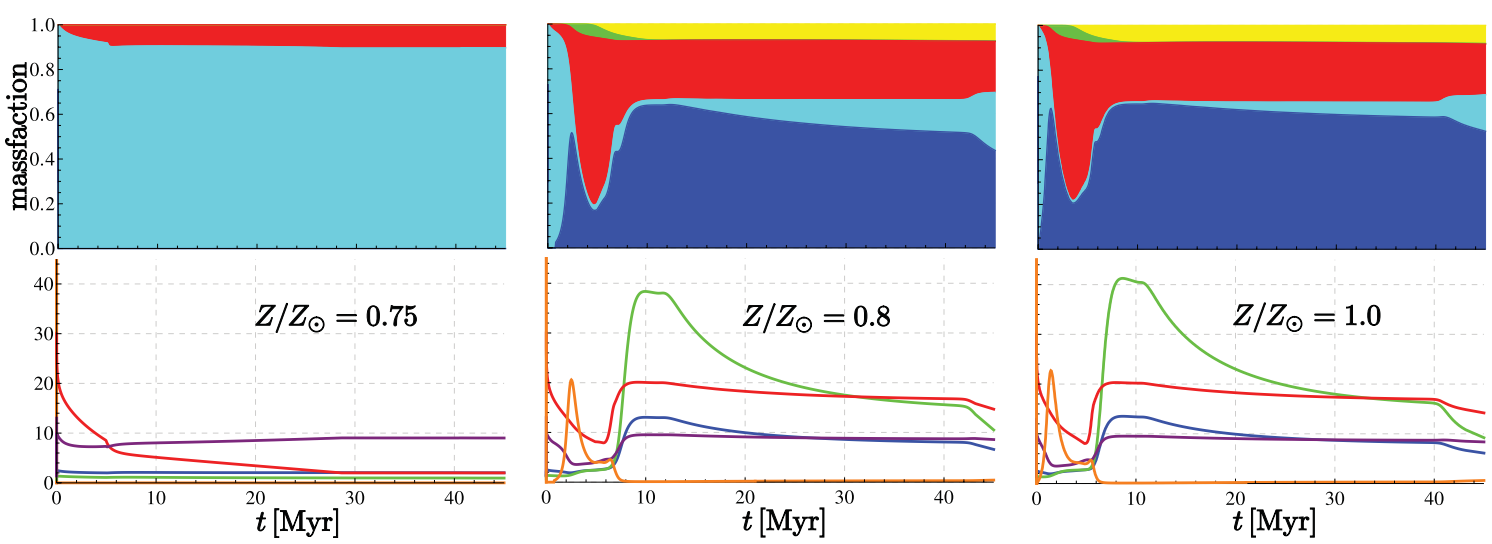

Figure 5. One-zone models for different metallicities at fixed initial density $n=75 \mathrm{~cm}^{-3}$ and moderate external driving $\mathcal{M}_{\Sigma}=0.2$. See the legend in Fig. 3 for a definition of the plotted quantities.

different metallicities $Z / Z_{\odot}=1.0,0.8$ and 0.75 , one can see that lowering $Z$ has effects on the evolution similar to those of lowering $n$. This is simply a consequence of the dependence of the following processes on the density of metals, which is proportional to $n Z$.

(i) $\Lambda_{\text {rad }}$ is primarily determined by metal-line cooling.

(ii) The major contribution to the $\mathrm{H}_{2}$ production rate (see equation 28) is the formation of molecules on dust grains, whose abundance is assumed to be proportional to $Z$.

(iii) The absorption of $\mathrm{H}_{2}$-dissociating radiation outside of molecular cores is dominated by dust extinction.

Consequently, $f_{\mathrm{c}, \mathrm{H}_{2}}$ and the relative mass fractions of phases are sensitive to $Z$, while quantities related to gravity are only indirectly affected. For this reason, $l_{\mathrm{c}}, t_{\mathrm{ff}}$ and $\mathrm{SFR}_{\mathrm{c}, \mathrm{ff}}$ remain almost unaffected when varying $Z$.

Next, we consider the influence of turbulence driving. Generally, a higher production rate $\Sigma$ increases the turbulent energy $e_{\mathrm{t}}$ and damps the peaks of star formation during the initial transient phase. Consequently, the stationary phase is entered earlier and more smoothly, as one can see in Fig. 6. Even in the absence of turbulent energy injection $\left(\mathcal{M}_{\Sigma}=0\right)$, the turbulence generated by the cooling instability and SNe plays an important role in limiting

the star formation rate (see the left-hand panel in Fig. 6). This is caused by a decrease of $\rho_{\mathrm{c}, \mathrm{pa}}$, as indicated by the growth of $l_{\mathrm{c}}$. Small $\mathcal{M}_{\Sigma}$ do not cause a major increase of $e_{\mathrm{t}}, \mathcal{M}_{\mathrm{c}}$ or $l_{\mathrm{c}}$ (middle panel of Fig. 6) compared to $\mathcal{M}_{\Sigma}=0$, although $u_{\mathrm{w}}$ settles at a slightly higher level due to the increased energy input. However, if the production of turbulence is dominated by $\Sigma$, the turbulent contribution to the effective pressure becomes comparable to the thermal pressure in the warm phase ( $e_{\mathrm{t}} \simeq u_{\mathrm{w}}$ in the right-hand panel of Fig. 6). As a consequence, the cold-phase pressure increases relative to the warm-phase pressure (see equation 9), and $\rho_{\mathrm{c}, \mathrm{pa}}$ decreases. This results in a lower star formation efficiency $\varepsilon_{\mathrm{ff}}$. Apart from this effect on $\varepsilon_{\mathrm{ff}}$ for $e_{\mathrm{t}} \sim u_{\mathrm{w}}$, turbulence also affects the molecular fraction $f_{\mathrm{c}, \mathrm{H}_{2}}$ and the star formation efficiency in the molecular gas, $\mathrm{SFR}_{\mathrm{c}, \mathrm{ff}}$. These subdominant effects are discussed in Section 6.3.

\subsection{Equilibrium star formation efficiency}

Although the transient phases discussed in Section 6.2 shed light on the complex interplay between the various physical processes contributing to the multi-phase dynamics, the approximately statistically stationary regimes of star formation are most relevant for applications. To numerically determine equilibrium solutions, we
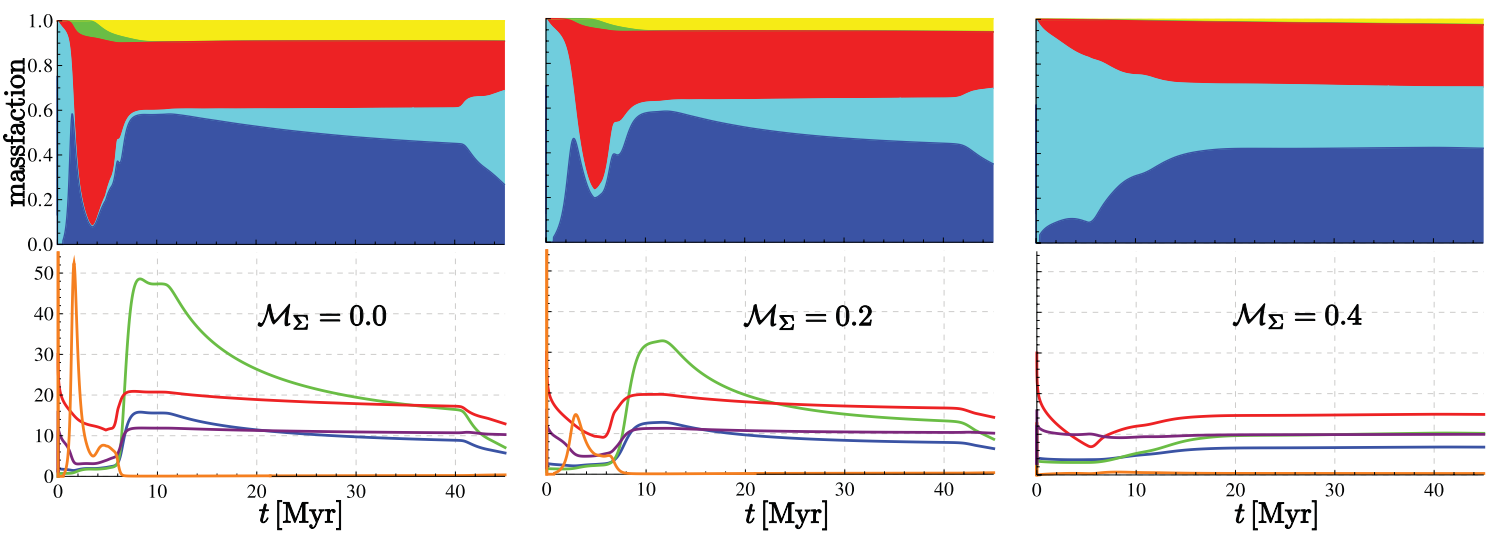

Figure 6. One-zone models for different rates of turbulent energy injection at fixed initial density $n=50 \mathrm{~cm}^{-3}$ and solar metallicity. See the legend in Fig. 3 for a definition of the plotted quantities.

(C) 2012 The Authors, MNRAS 421, 1838-1860

Monthly Notices of the Royal Astronomical Society (C) 2012 RAS 


\section{2}

\section{H. Braun and W. Schmidt}

assume a small star formation rate of 1 per cent as initial condition and integrate the rate equations until the relative temporal variance of $\dot{\rho}_{\mathrm{s}}$ over a feedback period ( $\gtrsim 40 \mathrm{Myr}$ ) becomes less than $10^{-4}$ or the star formation rate approaches zero (in the latter case the solution is not considered to be an equilibrium solution). We also check if the equilibrium conditions (81) and (82) are fulfilled with a relative accuracy better than $10^{-4}$. In contrast to the dynamical evolutions discussed before, we keep the total gas mass constant in the course of the integration, because we want to obtain equilibrium solutions for fixed gas densities that do not depend on the gas consumption by star formation during transient phases prior to the statistically stationary states. In numerical simulations, where the model describes a certain grid zone, one can think of the gas being replenished by neighbouring regions. Of course, such conditions will be met only to a certain degree and for a limited period of time. Nevertheless, the equilibrium solutions are useful to understand the behaviour of the system, and these solutions can be utilized as approximations to the star formation under quasi-stationary conditions.

\subsubsection{Gas density and metallicity}

Many star formation recipes used in astrophysical simulations assume, inspired by observational Kennicutt-Schmidt relations, that stars are formed with a fixed efficiency per free-fall time if a certain density threshold is exceeded. Our model shows such a behaviour under the condition of low turbulence driving $\Sigma$, as the star formation efficiency $\varepsilon_{\mathrm{ff}}$ saturates quickly above a metallicity-dependen density threshold (see Fig. 7, left-hand panel). The dependence of $\varepsilon_{\mathrm{ff}}$ on $n$ is governed by the molecular fraction $f_{\mathrm{c}, \mathrm{H}_{2}}$, while $\mathrm{SFR}_{\mathrm{c}, \mathrm{ff}}$ remains approximately constant for all $n \gg 1 \mathrm{~cm}^{-3}$. A similar dependence on the metallicity can be seen in Fig. 7 (right-hand panel). This is a consequence of cooling, $\mathrm{H}_{2}$ production and extinction of $\mathrm{H}_{2}$-dissociating radiation being mainly dependent on $n Z$.

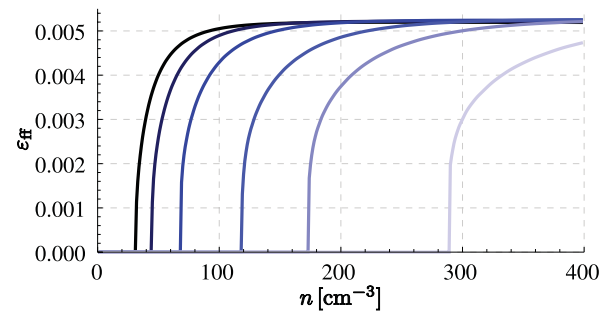

For the zone evolutions with constant total mass (gas and stars) shown in the left-hand panels of Figs 4 and 5, the star formation efficiency vanishes, although $n$ and $Z$ are above the threshold values for star formation following from Fig. 7. This can be understood as a consequence of the different initial conditions. Due to the lack of stellar feedback, the gas phases are never pushed into a star-forming regime in the former calculations. In a numerical simulation, the heating of gas by nearby stars could trigger star formation in so far inactive gas. Otherwise, the gas will remain cold and inactive as long as it does not become dense enough to form molecular cores and to start star formation.

\subsubsection{External turbulence driving}

Low turbulence intensity maintained by external driving $\left(\mathcal{M}_{\Sigma} \lesssim\right.$ $0.2)$ does not change the shape of $\varepsilon_{\mathrm{ff}}(n)$ appreciably. Fig. 8 (lefthand panel) shows that moderate values of $\mathcal{M}_{\Sigma}$ increase the density threshold and lower the saturation level of $\varepsilon_{\mathrm{ff}}$ slightly. As $\mathcal{M}_{\Sigma}$ approaches unity, however, star formation becomes more and more inhibited. Turbulence also smears out the density threshold. In Fig. 8 (right-hand panel), four regimes can be identified, in which the effects of turbulence injection differ with increasing $\mathcal{M}_{\Sigma}$.

(i) For small $\mathcal{M}_{\Sigma}$, the additional heating of the gas by turbulent dissipation partially counters the effects of turbulence suppressing star formation that become dominant for stronger $\mathcal{M}_{\Sigma}$.

(ii) For higher $\mathcal{M}_{\Sigma}$, the production of $e_{\mathrm{t}}$ is dominated by the turbulent cascade and $e_{\mathrm{t}}$ roughly follows $\mathcal{M}_{\Sigma}^{2}$. As a consequence, turbulent pressure contributes significantly to the pressure balance between the phases, and $\rho_{\mathrm{c}, \mathrm{pa}}$ decreases $\left(\rho_{\mathrm{c}, \mathrm{pa}} \rightarrow \rho\right.$ in the limit of large $e_{\mathrm{t}}$ ). The lowering of the cold-gas density results in the steep reduction of $\varepsilon_{\mathrm{ff}}$ as $\mathcal{M}_{\Sigma}$ rises.

(iii) For stronger turbulence intensity, $\rho_{\mathrm{c}, \mathrm{pa}}$ is low but the turbulent broadening of the density pdf becomes important (enhancement

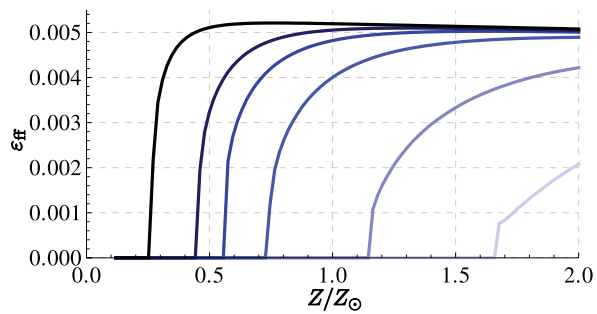

Figure 7. Equilibrium star formation efficiency per free-fall time, $\varepsilon_{\mathrm{ff}}$, versus number density $n$ for different metallicities $Z / Z_{\odot}=1.0,0.8,0.6,0.4$ and 0.2 from dark to light blue (left-hand panel), and $\varepsilon_{\mathrm{ff}}$ as a function of $Z / Z_{\odot}$ for different $n=200,100,75,50,25$ and $12.5 \mathrm{~cm}^{-3}$ from dark to light blue (right-hand panel)
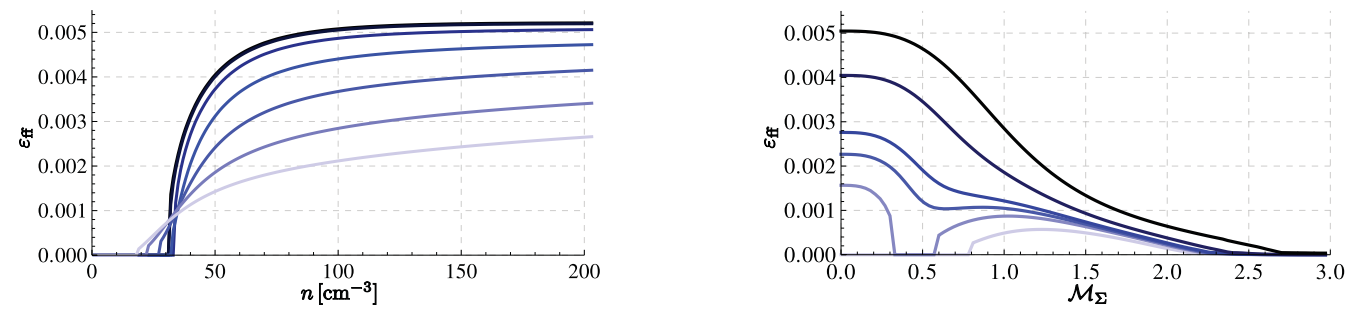

Figure 8. Equilibrium star formation efficiency per free-fall time, $\varepsilon_{\mathrm{ff}}$, versus number density $n$ for different turbulent energy injection rates $\Sigma$ (left-hand panel) in terms of $\mathcal{M}_{\Sigma}=0.0,0.2,0.4,0.6,0.8,1.0$ and 1.2 from dark to light blue (see equation 89), and $\varepsilon_{\mathrm{ff}}$ as a function of $\mathcal{M}_{\Sigma}$ for different number densities $n=27.25,32.5,35,37.5,50$ and $100 \mathrm{~cm}^{-3}$ from light to dark blue (right-hand panel) 

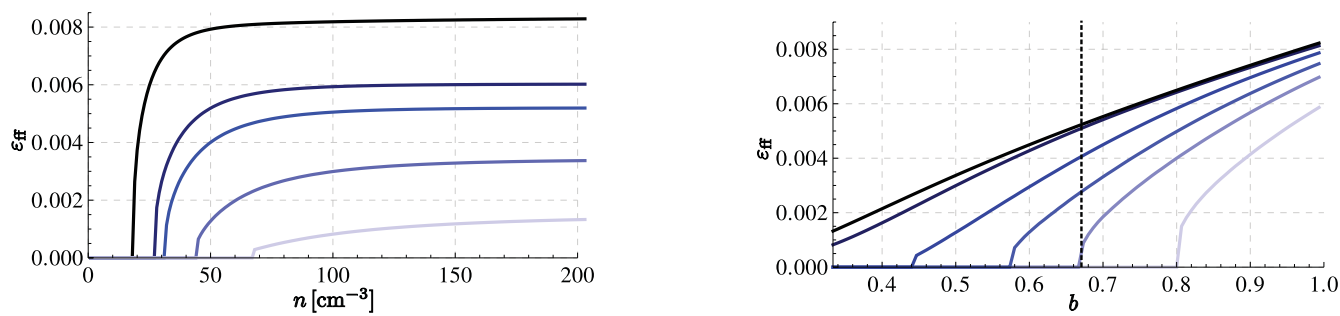

Figure 9. Equilibrium star formation efficiency per free-fall time, $\varepsilon_{\mathrm{ff}}$, versus number density $n$ for different values of the compressive factor $b$ in the cold phase (left-hand panel). From light to dark blue, $b=1 / 3,0.5,2 / 3,0.75$ and 1.0. Here, $\varepsilon_{\mathrm{ff}}$ over turbulence forcing parameter $b$ (right-hand panel) for the number densities $n=200,100,50,37.5,31.25$ and $25 \mathrm{~cm}^{-3}$ (from dark to light blue). The default value $b=2 / 3$ is marked by the dotted line.
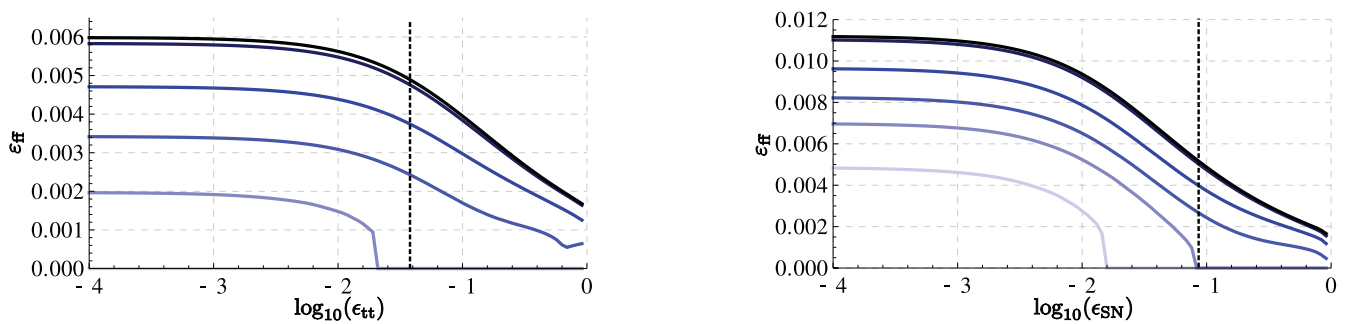

Figure 10. Equilibrium star formation efficiency per free-fall time, $\varepsilon_{\mathrm{ff}}$, versus the turbulent production efficiencies $\epsilon_{\mathrm{tt}}$ (left-hand panel) and $\epsilon_{\mathrm{SN}}$ (right-hand panel) for the number densities $n=200,100,50,37.5,31.25$ and $25 \mathrm{~cm}^{-3}$ (from dark to light blue). The default values $\left(\epsilon_{\mathrm{tt}}=0.025\right.$ and $\left.\epsilon_{\mathrm{SN}}=0.085\right)$ are marked by the dotted lines.

of $\mathrm{H}_{2}$ production by the clumping factor $C_{\rho}$ and dependence of $\mathrm{SFR}_{\mathrm{c}, \mathrm{ff}}$ on the pdf; see Section 3.1). This effect can clearly be seen for initial densities lower than $50 \mathrm{~cm}^{-3}$, where a second maximum of $\varepsilon_{\mathrm{ff}}$ can be discerned. This is the regime in which star formation critically depends on the properties of self-gravitating turbulence.

(iv) If $\mathcal{M}_{\Sigma}$ increases further, the growth of the minimum overdensity for star formation in the cold gas, $x_{\text {crit }} \propto e_{t}^{2}$ (see equations 20 and 25) dominates, and $\varepsilon_{\mathrm{ff}}$ asymptotically falls off to zero.

Because of the dependence of $\mathrm{SFR}_{\mathrm{c}, \mathrm{ff}}$ and $f_{\mathrm{c}, \mathrm{H}_{2}}$ on the density pdf, the saturation level of $\varepsilon_{\mathrm{ff}}$ and the density threshold are significantly affected by the weight of compressive forcing modes relative to solenoidal modes. Assuming that the width of the density pdf is given by $\sigma \approx \log \left(1+\mathcal{M}_{\mathrm{c}}^{2} b^{2}\right)$, where $\mathcal{M}_{\mathrm{c}}$ is the rms Mach number of turbulent motions in the cold gas and $b$ varies between $1 / 3$ for purely solenoidal forcing and 1 for purely compressive forcing, we obtain the equilibrium solutions plotted in Figs 9 and 10 (right-hand panel). The nature of turbulence driving in the ISM is still a matter of debate. Moreover, the mixture of solenoidal and compressive modes is likely to be scale dependent. Here, we adopt the intermediate value $b=2 / 3$ as default.

As explained in Section 5.2, turbulence generally decreases the temperature of the warm gas in equilibrium (see equation 86). Fig. 11 shows that this is roughly a 10 per cent effect for densities higher than the threshold for star formation. In a certain sense, this is the deviation from the $\mathrm{SH} 03$ equilibrium solution $u_{\mathrm{SH}}$ (see equation 87). However, the value of $u_{\mathrm{SN}}$ used by SH03 and the coefficient of the first term is different from our definition, so the actual difference is greater. As one can see in Fig. 11, there is a noticeable deviation even without external turbulence driving. This effect is caused by internal turbulence driving, which is considered next.

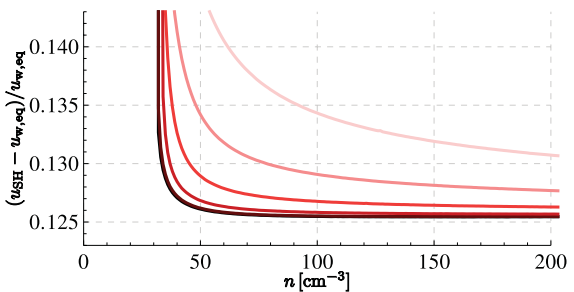

Figure 11. Relative deviation of the equilibrium solution for the warm-gas energy, $u_{\mathrm{w}, \text { eq }}$, from the first two terms on the right-hand side of equation (86), corresponding to zero turbulent energy. The different curves are obtained for $\mathcal{M}_{\Sigma}=0.0,0.2,0.4,0.6,0.8$ and 1.0 from dark to light red.

\subsubsection{Thermal instability and stellar feedback}

If the energy transfer from larger scales is small, internal driving by the thermal instability and stellar feedback dominate the production of turbulence. In this case, $e_{\mathrm{t}}$ is mainly controlled by the turbulence production efficiencies $\epsilon_{\mathrm{tt}}$ and $\epsilon_{\mathrm{SN}}$. An increase of $\epsilon_{\mathrm{tt}}$ lowers the thermal energy of the warm gas, $u_{\mathrm{w}}$, as the cooling instability transfers thermal energy to turbulence more efficiently. For higher $\epsilon_{\mathrm{SN}}$, less energy is deposited by $\mathrm{SNe}$ in the warm phase and also more turbulent energy is produced, which tends to decrease the star formation rate. However, the effect of $\epsilon_{\mathrm{SN}}$ is limited for high $\epsilon_{\mathrm{tt}}$ because the increased production of turbulent energy by the thermal instability reduces star formation and, consequently, supernova feedback. On the other hand, only a relatively small fraction of the thermal energy of the warm phase can be converted into turbulent energy by the thermal instability without violating the second law of thermodynamics. This suggests that $\epsilon_{\mathrm{tt}}$ has to be small compared to unity. 


\section{H. Braun and W. Schmidt}

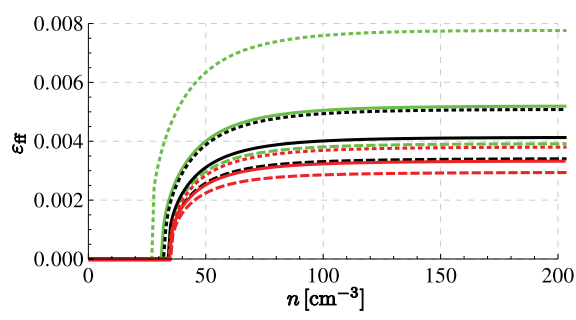

Figure 12. Equilibrium star formation efficiency per free-fall time, $\varepsilon_{\mathrm{ff}}$, versus number density $n$ for different values of the turbulence production efficiencies $\epsilon_{\mathrm{tt}}$ and $\epsilon_{\mathrm{SN}}: \epsilon_{\mathrm{tt}}=0.025$ (green), 0.085 (black), 0.17 (red), and $\epsilon_{\mathrm{SN}}=0.025$ (dotted), 0.085 (solid) and 0.17 (dashed).

Since turbulence produced by SNe on length-scales much smaller than $l$ is rapidly dissipated into thermal energy, only the fraction specified by $\epsilon_{\mathrm{SN}}$ effectively enters the turbulent energy $e_{\mathrm{t}}$, while the fraction $1-\epsilon_{\mathrm{SN}}$ is immediately turned into thermal energy. This implies that $\epsilon_{\mathrm{SN}}$ is scale dependent. For $l$ greater than a few parsec, $\epsilon_{\mathrm{SN}}$ must not be lower than a few percent. Otherwise, the star formation efficiency would become significantly greater than 0.1 , in contradiction to the majority of observations (Krumholz \& Tan 2007; Murray 2011). The average total energy deposited by a supernova in the ISM is $E_{\mathrm{SN}} \approx 10^{51} \mathrm{erg}$. Roughly, $8.5 \times 10^{49} \mathrm{erg}$ of this energy enters the ISM in form of kinetic energy or, from the perspective of our model, in form of turbulent energy (Thornton et al. 1998). Hence, we adopt the value $\epsilon_{\mathrm{SN}}=0.085$ as default The left and right panels of Fig. 10 show that $\varepsilon_{\mathrm{ff}}$ saturates if one efficiency is much greater than the other. The effect of $\epsilon_{\mathrm{tt}}$ and $\epsilon_{\mathrm{SN}}$ saturates slowly for large values of them (Fig. 12) is a consequence of $\rho_{\mathrm{c}, \mathrm{pa}} \rightarrow \rho$, if turbulent energy is efficiently produced by whatever mechanism. To obtain a plausible star formation efficiency, we choose $\epsilon_{\mathrm{tt}}=0.025$.

With the number of SNe per solar mass of formed stars,

$n_{\mathrm{SN}}=\frac{1}{M_{*}} \int_{8 \mathrm{M}_{\odot}}^{40 \mathrm{M} \odot} \frac{\mathrm{d} N}{\mathrm{~d} m_{*}} \mathrm{~d} m_{*}$,

we estimate the feedback energy per solar mass,

$u_{\mathrm{SN}}=n_{\mathrm{SN}} E_{\mathrm{SN}} \frac{1-\beta}{\beta} \approx 6 \times 10^{49} \operatorname{erg~}_{\odot}^{-1}$,

with the feedback fraction $\beta$ as defined in equation (85). This value is subject to large uncertainties. However, Fig. 13 shows that the star formation efficiency is relatively robust against variations in $u_{\mathrm{SN}}$ if its value is at least the same order of magnitude as the above default value for the model.

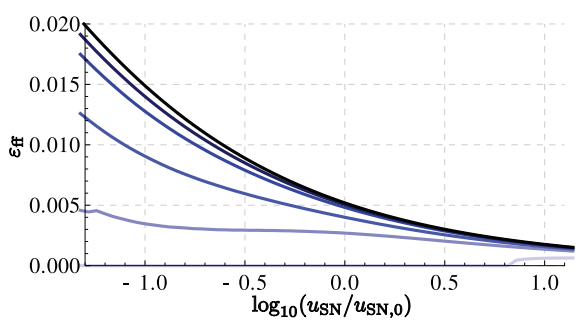

Figure 13. Equilibrium star formation efficiency per free-fall time, $\varepsilon_{\mathrm{ff}}$, versus logarithmic specific energy of $\mathrm{SN} \log \left(u_{\mathrm{SN}} / u_{\mathrm{SN}, 0}\right)$, where $u_{\mathrm{SN}, 0}=$ $6 \times 10^{49} \mathrm{erg} \mathrm{M}_{\odot}^{-1}$, for $n=25,37.5,50,75,100$ and $200 \mathrm{~cm}^{-3}$ from light to dark blue.

\subsubsection{Scale dependence}

For length-scales $l \gtrsim 10 \mathrm{pc}$, the star formation efficiency is almost exactly scale invariant (see Fig. 14). This demonstrates that even without maintaining a scale-invariant rate of energy injection by external turbulence forcing, the system settles into an equilibrium state, for which the clump length-scale $l_{\mathrm{c}}$ and the Mach number of turbulence in the cold-gas phase, $\mathcal{M}_{\mathrm{c}}$, are nearly independent of $l$. Internal driving and turbulent dissipation regulate $e_{\mathrm{t}}$ such that the scaling of the turbulent velocity fluctuations from $l$ to $l_{\mathrm{c}}$ by the power law (12) results in a fixed $\mathcal{M}_{\mathrm{c}} \propto \sigma_{\mathrm{c}}$, regardless of the choice of $l$. However, the equilibrium is influenced by the scaling parameter $\eta$. For weakly compressible turbulence (up to Mach numbers around unity), $\eta$ is close to the Kolmogorov value $1 / 3$, whereas $\eta$ rises to $1 / 2$ for supersonic turbulence. The dependence of $\varepsilon_{\mathrm{ff}}$ on $\eta$ is shown in Fig. 15. Since we assume that turbulence is supersonic within the cold clumps, but transonic in the warm gas, and there are only little changes of $\varepsilon_{\mathrm{ff}}$ if $\eta$ is about $1 / 3$, we set $\eta=1 / 3$ as a reasonable approximation if $l$ is greater than $l_{\mathrm{c}}$.

If $l$ is only a few parsec or less, on the other hand, $l_{\mathrm{c}}$ may exceed $l$. In this case, basic assumptions of the model break down, as the notion of cold-gas clumps in pressure balance with the warm gas in the reference volume of size $l$ becomes meaningless. The percolation of the cold phase and the transition to a one-phase medium is not yet implemented in the model (see Section 5.1). If the model in its present form is applied as an SGS model in AMR simulations, a maximum refinement limit has to be applied such that $l_{\mathrm{c}}<l$ is ensured. In cosmological simulations with $l_{\text {min }} \sim 100 \mathrm{pc}$, this condition will almost certainly be satisfied. For high-resolution simulations of individual galaxies with $l_{\min } \sim 1 \mathrm{pc}$, however, situations where cold-gas regions extend over several zones cannot be avoided. Then a viable model has to deal with the transition to a one-phase medium.

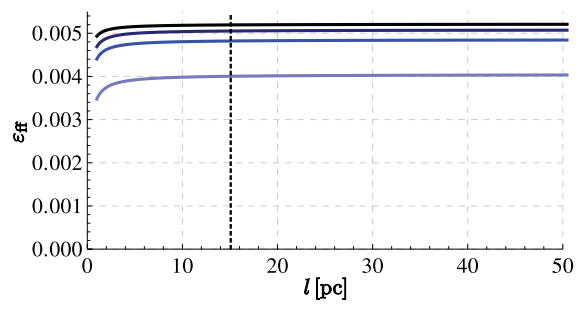

Figure 14. Equilibrium star formation efficiency per free-fall time, $\varepsilon_{\mathrm{ff}}$, versus length-scale $l$ for different number densities $n=50,75,100$ and $200 \mathrm{~cm}^{-3}$ (from light to dark blue), without external driving.

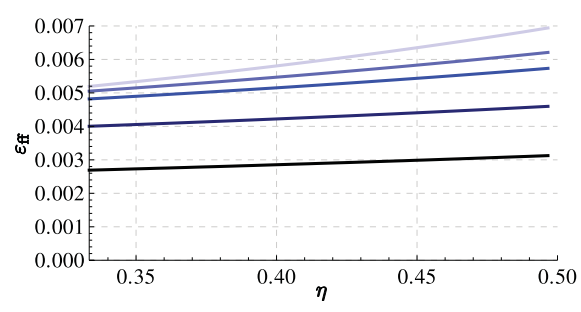

Figure 15. Equilibrium star formation efficiency per free-fall time, $\varepsilon_{\mathrm{ff}}$, versus the turbulence scaling exponent $\eta$ for $n=37.5,50,75,100$ and $200 \mathrm{~cm}^{-3}$ from dark to light blue.

(c) 2012 The Authors, MNRAS 421, 1838-1860 Monthly Notices of the Royal Astronomical Society (C) 2012 RAS 

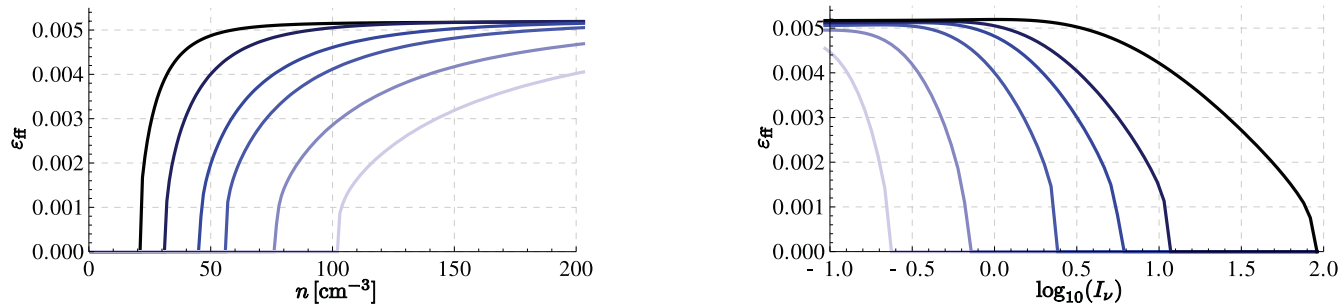

Figure 16. Equilibrium star formation efficiency per free-fall time, $\varepsilon_{\mathrm{ff}}$, versus number density $n$ for the normalized interstellar radiation field $I_{v}=0.5$, $1.0,1.5,2.0$ and 3.0 from dark to light blue (left-hand panel). The metallicity of the gas is $Z=0.5 \mathrm{Z}_{\odot}$, and $\varepsilon_{\mathrm{ff}}$ as a function of $\log \left(I_{\nu}\right)$ for $n=$ $25,50,75,100$ and $200 \mathrm{~cm}^{-3}$ from light to dark blue (right-hand panel). The default $I_{v}=1$ is marked by the vertical dotted line.
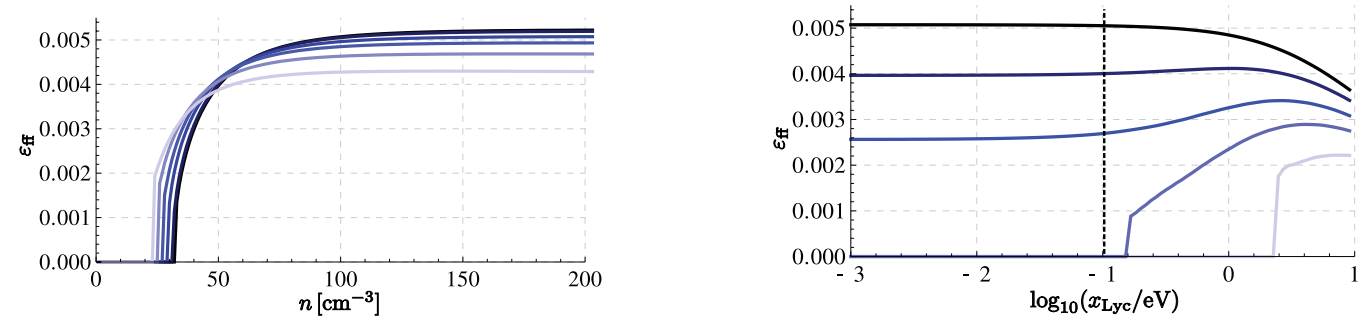

Figure 17. Equilibrium star formation efficiency per free-fall time, $\varepsilon_{\mathrm{ff}}$, versus number density $n$ for $x_{\mathrm{Lyc}}=0.01,0.1,0.5,1.0,2.0$ and $4.0 \mathrm{eV}$ photon ${ }^{-1}$ from dark to light blue (left-hand panel), and $\varepsilon_{\mathrm{ff}}$ as a function of $\log \left(x_{\mathrm{Lyc}} / \mathrm{eV}\right)$ for different $n=25,26.5,28.125,31.25,37.5,50$ and $100 \mathrm{~cm}^{-3}$ from light to dark blue (right-hand panel). The default $x_{\mathrm{Lyc}}=0.1 \mathrm{eV}$ is marked by the dotted line.

\subsubsection{Photodissociation and heating}

The intensity of the ambient interstellar radiation field $I_{v}$ (in units of the Draine field) is an external parameter and depends on the environment of the reference volume, i.e. the location and structure of the host galaxy and its surroundings. Thus, it is a parameter at the same level as $n, Z$ and $\mathcal{M}_{\Sigma}$, which has a direct impact on $f_{\mathrm{c}, \mathrm{H}_{2}}$ and $u_{\mathrm{w}}$ via $\mathrm{H}_{2}$ dissociation and photoelectric heating. Fig. 16 demonstrates that the density threshold of star formation and the shape of $\varepsilon_{\mathrm{ff}}(n)$ change significantly with variations in $I_{v}$, while the saturation level of $\varepsilon_{\mathrm{ff}}$ remains nearly unaffected. Consequently, the threshold density of star formation is mainly determined by $Z$ and $I_{\nu}$.

The heat deposited in the gas per Lyc photon, $x_{\mathrm{Lyc}}$, depends on the shape of the spectrum emitted by the young massive stars and the state of the absorbing gas. Both gas phases are affected by Lyc heating (see Section 4.1), and it reduces the amount of warm gas dropping into the cold phase if the cooling instability is active. As a consequence, an increase of $x_{\mathrm{Lyc}}$ lowers the level of saturation of $\varepsilon_{\mathrm{ff}}(n)$ significantly (see Fig. 17). Even for small $x_{\text {Lyc }}$, the density threshold of star formation is affected by the influence of Lyc heating on the thermal pressure of the warm phase and, thus, on $\rho_{\text {c.pa }}$. Since the cross-section decreases significantly for large excess energies above the Rydberg energy and inelastic scattering processes become more likely, $x_{\mathrm{Lyc}}>0.5 \mathrm{eV}$ is not plausible. We use $x_{\mathrm{Lyc}}=0.1 \mathrm{eV}$ as default value.

\subsubsection{Clump collisions}

The evaporation of clumps due to collisions lowers the $\rho_{\mathrm{c}} / \rho_{\mathrm{w}}$ ratio, but the clumps are typically too small and collisions are too rare to influence the evolution significantly even for a high efficiency $\epsilon_{\mathrm{cc}}$.

(C) 2012 The Authors, MNRAS 421, 1838-1860

Monthly Notices of the Royal Astronomical Society (C) 2012 RAS
If $l_{\mathrm{c}}$ becomes comparable to $l$, clump collisions cannot be applied for obvious reasons. We thus neglect this effect altogether by setting $\epsilon_{\mathrm{cc}}=0$.

\subsubsection{Prestellar mass-loss}

As mentioned by Chabrier \& Hennebelle (2010), the core mass function (CMF; the observed mass function of gravitationally bound cores in molecular clouds) and the IMF (the IMF of stars) are similar, except for an almost mass-independent shift by factor about 2-3 (also see Matzner \& McKee 2000). We account for the mass reduction due to the evolution from the CMF to the IMF by reducing the star formation efficiency by a factor of $1-f_{\text {loss }}$, where $f_{\text {loss }}$ is interpreted as the mass fraction that is ejected during the collapse prior to star formation. As one can see in Fig. 18, increasing $f_{\text {loss }}$ reduces the star formation rate significantly. A good agreement with observational relations is obtained for the intermediate value $f_{\text {loss }}=0.6$.

\subsection{Comparison to observations}

Comparisons of one-zone results with observations are difficult, mainly for the following reasons. First, the conversion of the modelled volume densities into the corresponding surface densities is non-trivial. Secondly, star-forming regions are generally not in local star formation equilibrium, which could explain the breakdown of Kennicutt-Schmidt relations on small scales (e.g. Onodera et al. 2010; Schruba et al. 2010; Murray 2011). Without the detailed dynamic environment in a hydrodynamic simulation, we can only draw conclusions on the basis of the equilibrium solutions calculated with our model. Even so, we are able to demonstrate that these 

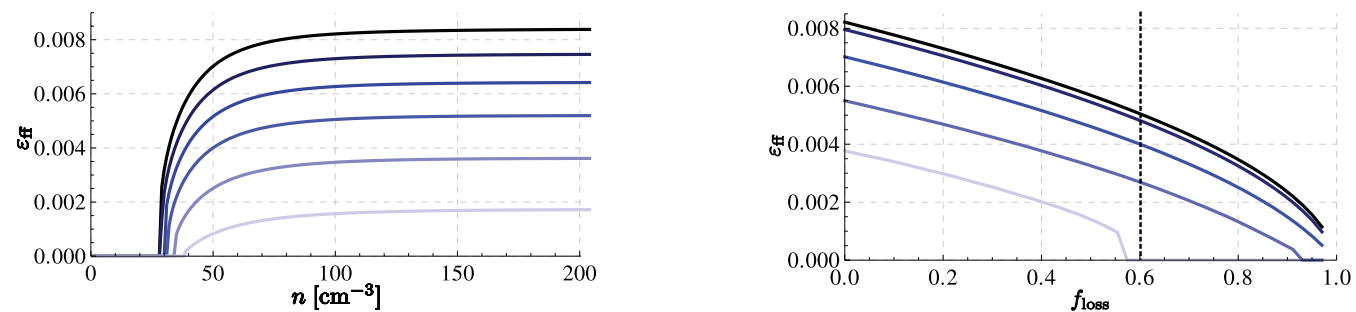

Figure 18. Equilibrium star formation efficiency per free-fall time, $\varepsilon_{\mathrm{ff}}$, versus number density $n$ for $f_{\text {loss }}=0.0,0.2,0.4,0.6,0.8$ and 0.95 from dark to light blue (left-hand panel), and $\varepsilon_{\mathrm{ff}}$ as a function of $f_{\text {loss }}$ for different $n=31.25,37.5,50,75$ and $100 \mathrm{~cm}^{-3}$ from light to dark blue (right-hand panel). The default $f_{\text {loss }}=0.5$ is marked by the dotted line.

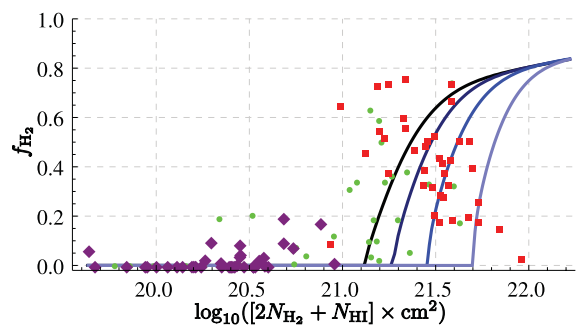

Figure 19. Molecular fraction $f_{\mathrm{H}_{2} \text {,tot }}$ versus logarithmic total hydrogen surface number density $\log \left(2 N_{\mathrm{H}_{2}}+N_{\mathrm{HI}}\right)$ in star formation equilibrium for different $Z / Z_{\odot}=1.0,0.8,0.6$ and 0.4 from dark to light blue. Red squares (Rachford et al. 2002, 2009), green circles (Wolfire et al. 2008) and purple diamonds (Gillmon et al. 2006) represent observations of translucent clouds in the Galaxy.

solutions are consistent with the constraints set by observations on kiloparsec scales.

Since the calculation of $f_{\mathrm{c}, \mathrm{H}_{2}}$ in our model only treats molecular hydrogen in cold clumps shielded from UV radiation, we neglect the molecular hydrogen in radiation-dominated areas, where a certain amount exists in equilibrium between production and radiative destruction. As a consequence, the predicted molecular hydrogen mass might be systematically too low. This discrepancy can become particularly strong in the case of low (column) densities. To estimate the hydrogen column density, we set $\left(2 N_{\mathrm{H}_{2}}+N_{\mathrm{HI}}\right) \simeq X \rho l / m_{\mathrm{H}}$. Fig. 19 shows that the transition from marginal to significant total molecular fractions $f_{\mathrm{H}_{2} \text {,tot }}$ occurs at column densities that are in good agreement with observations of translucent clouds in the Milky Way.

Observed star formation surface densities and depletion timescales, as well as the related surface densities of molecular and atomic hydrogen, are projected quantities. In the simplest case of a face-on galactic disc, these quantities are averaged over the thickness of the disc and over a certain solid angle (or area). Since we cannot account for spatial structures in the equilibrium one-zone models, we plot the star formation density over the atomic, total and molecular volume densities (with helium and metals included) for different metallicities and varying external driving in Figs 20 , 21 and 22, respectively. However, these plots should reproduce observed trends because the typical thickness of a star-forming region is of the order of $\gtrsim 10 \mathrm{pc}$, which is comparable to the typical lengthscale $\ell$ of our models, and the gas in star-forming regions contributes most to column densities. Indeed, in comparison to observational rates and densities (e.g. Bigiel et al. 2008; Schruba et al. 2011, fig. 4 and fig. 11, respectively), we find a shift in numbers by a factor $\gtrsim 10,{ }^{4}$ in both directions, but the general behaviour is very similar.

Fig. 20 shows that the star formation rate is clearly not correlated to $\mathrm{HI}$ densities below $\lesssim 1 \mathrm{M}_{\odot} \mathrm{pc}^{-3}$ (roughly corresponding to column densities $\lesssim 10 \mathrm{M}_{\odot} \mathrm{pc}^{-2}$ ), and higher densities averaged over the cold and warm phases are atypical. The star formation rate as a function of the total gas density (see Fig. 21) switches from zero at low densities, for which the fraction of $\mathrm{H}_{2}$ is negligible, to a tight correlation above $\sim 1 \mathrm{M}_{\odot} \mathrm{pc}^{-3}$. This threshold is caused by the transition from atomic to shielded molecular gas. Correspondingly, the star formation rate mainly correlates with the $\mathrm{H}_{2}$ density, which agrees with the KMT09 model. For a given set of parameters, the molecular gas depletion time-scale $t_{\mathrm{dep}, \mathrm{H}_{2}}=\dot{\rho}_{\mathrm{s}} /\left(f_{\mathrm{c}, \mathrm{H}_{2}} \rho_{\mathrm{c}}\right)$ varies only little over two orders of magnitude in $\mathrm{H}_{2}$ density (see Fig. 22) While the model predicts a depletion time of $\sim 0.7 \mathrm{Gyr}$ if internal driving is the dominant mechanism of turbulence production, the depletion time-scale increases significantly for external turbulence driving. For example, Bigiel et al. (2011) find $\sim 2$ Gyr observationally, which could be maintained by external driving for $\mathrm{H}_{2}$ densities up to a few $\mathrm{M}_{\odot} \mathrm{pc}^{-3}$. However, as we underestimate the $\mathrm{H}_{2}$ content in the gas, the depletion time tends to be too low in our model towards lower densities so that less external energy injection might be required to obtain the observed depletion time. A further caveat is the assumption of equilibrium.

Eventually, only the application as an SGS model in numerical simulations of galaxies will enable us to calculate relations between the star formation rate and the surface density by projecting the computed density fields. Since star-forming regions go through different evolutionary stages, during which star formation occurs episodically, the star formation rate has to be integrated in time to incorporate non-equilibrium effects into the depletion time-scale.

\section{DISCUSSION AND CONCLUSIONS}

In this paper, we propose a model for the multi-phase ISM and star formation, considering the effects of turbulence and stellar feedback. Based on the concept of Springel \& Hernquist (2003), we split the gas content of a region of given size into a two distinct fractions, representing a diffuse warm and a clumpy cold component. However, our model goes significantly beyond their approach. By applying a simplified treatment of molecular hydrogen formation and destruction, we relate the star formation rate to the fractional density

${ }^{4}$ The density plots are in units of $\mathrm{M}_{\odot} \mathrm{pc}^{-3}$ and $\mathrm{M}_{\odot} \mathrm{Myr}^{-1} \mathrm{pc}^{-3}$, while the observational column density plots are usually in units of $\mathrm{M}_{\odot} \mathrm{pc}^{-2}$ and $\mathrm{M}_{\odot} \mathrm{yr}^{-1} \mathrm{kpc}^{-3}$

(C) 2012 The Authors, MNRAS 421, 1838-1860 Monthly Notices of the Royal Astronomical Society (C) 2012 RAS 

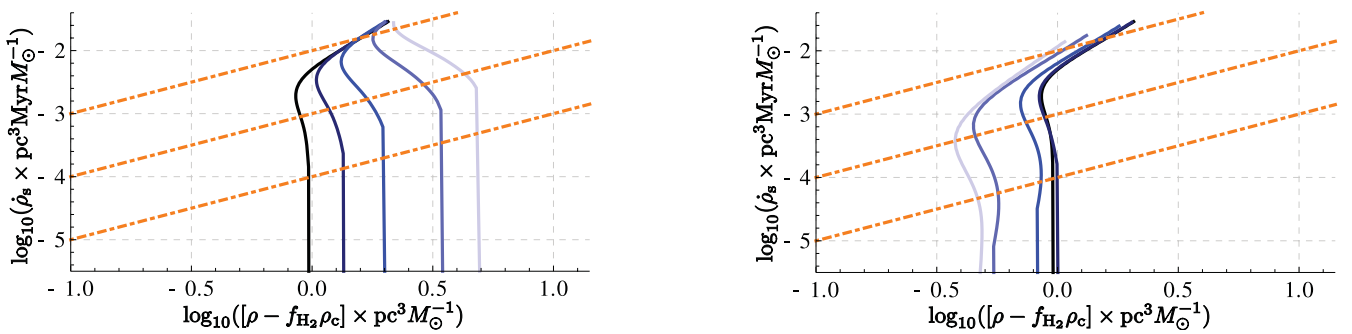

Figure 20. Star formation rate as a function of the fractional atomic density $\rho-f_{\mathrm{c}, \mathrm{H}_{2}} \rho_{\mathrm{c}}$ for $Z / \mathrm{Z}_{\odot}=1.0,0.8,0.6,0.4$ and0.3 from dark to light blue (left-hand panel) and for different turbulent energy injection rates $\Sigma$ in terms of $\mathcal{M}_{\Sigma}=0.0,0.4,0.8,1.2$ and 1.4 (from dark to light blue; right-hand panel). The long- and short-dashed lines indicate the asymptotes with slopes $1.4\left(\mathcal{M}_{\Sigma}=0.0\right.$, all $\left.Z / Z_{\odot}\right)$ and $1.6\left(\mathcal{M}_{\Sigma}=1.0\right)$, respectively. The orange dot-dashed lines mark fixed depletion time scales of $0.1,1$ and $10 \mathrm{Gyr}$ from the top to the bottom of the graph.
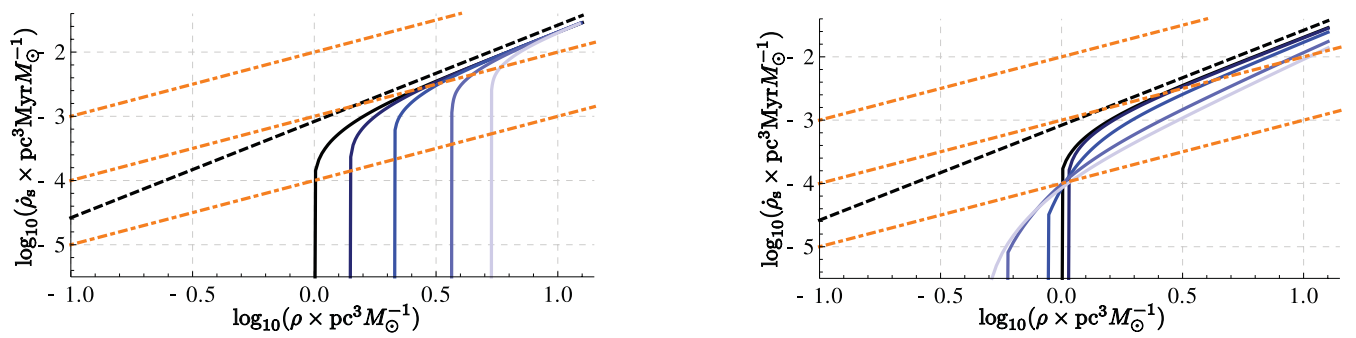

Figure 21. Star formation rate as a function of the total density $\rho$ for $Z / Z_{\odot}=1.0,0.8,0.6,0.4$ and 0.3 from dark to light blue (left-hand panel) and for different turbulent energy injection rates $\Sigma$ in terms of $\mathcal{M}_{\Sigma}=0.0,0.4,0.8,1.2$ and 1.4 (from dark to light blue; right-hand panel). The Kennicutt-Schmidt asymptote with slope 1.5 is indicated by the dashed line, orange dot-dashed lines as in Fig. 20.
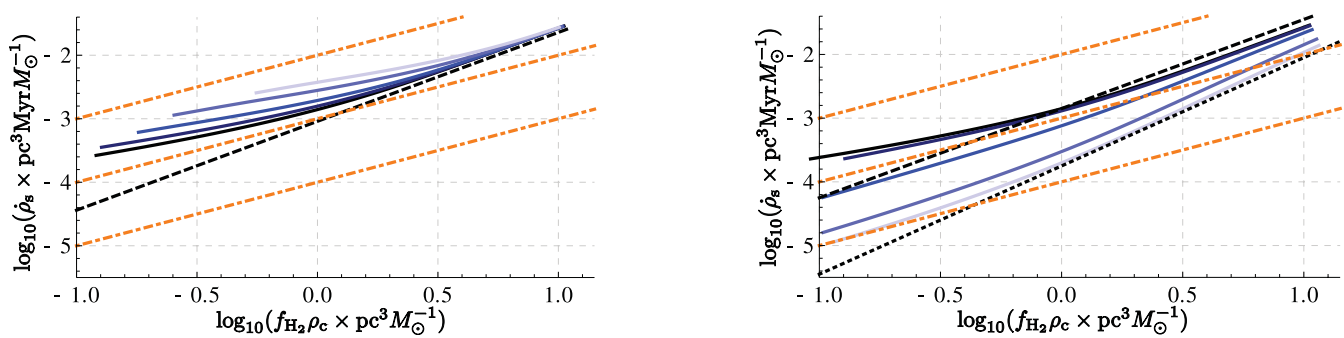

Figure 22. Star formation rate as a function of the fractional molecular density $f_{\mathrm{c}, \mathrm{H}_{2}} \rho_{\mathrm{c}}$ for $\mathrm{Z} / \mathrm{Z}_{\odot}=1.0,0.8,0.6,0.4$ and 0.3 from dark to light blue (left-hand panel) and for different turbulent energy injection rates $\Sigma$ in terms of $\mathcal{M}_{\Sigma}=0.0,0.4,0.8,1.2$ and 1.4 (from dark to light blue; right-hand panel). The long- and short-dashed lines indicate the asymptotes with slopes $1.4\left(\mathcal{M}_{\Sigma}=0.0\right.$, all $\left.Z / Z_{\odot}\right)$ and $1.6\left(\mathcal{M}_{\Sigma}=1.0\right)$, respectively. Orange dot-dashed lines are as in Fig. 20.

of molecular hydrogen in the cold-gas phase (KMT09). While star formation models that are applied in numerical simulations usually assume a constant efficiency parameter that is globally calibrated against the Kennicutt-Schmidt law, we dynamically calculate the star formation efficiency on the basis of local physical processes. In the spirit of KM05, star formation is regulated by the virial parameter and the turbulent velocity dispersion of cold clumps. The interrelationship in our model is more elaborate though. Turbulent energy can be produced by a turbulent cascade from larger scale, but also via internal driving by the thermal instability of the gas and by supernova feedback. To determine the gas fraction that collapses per free-fall time-scale into stars, we assume a lognormal distribution of density fluctuations in the cold gas, and relate the critical density for a gravitational collapse to the virial parameter and the turbulent Mach number on the typical length-scale of the cold clumps PN11.
To account for the effect of SNe, we use a delayed feedback model. Apart from the turbulent energy, the fractional densities of the cold and warm phases and the thermal energy of warm gas are evolved (the temperature of the cold gas is assumed to be constant). Mass and energy is exchanged between the phases via radiative cooling, heating and mixing processes. An important source of heating is the stellar population in the volume. We consider two feedback mechanisms, taking the time-scales of stellar evolution into account: the Lyc emission and SN II explosions of young massive stars. To close the system of equations, we assume an effective (i.e. thermal plus turbulent) pressure balance between the cold clumps and the surrounding warm gas.

By integrating the evolutionary equations for the averaged mass fractions and energies in a given spatial volume, we have obtained semi-analytical one-zone models, with the total gas density $n$, the 


\section{8}

\section{H. Braun and W. Schmidt}

metallicity $Z$ and the rate of energy injection by external turbulence forcing, $\Sigma$, as main parameters. Of particular interest are equilibrium solutions with a constant star formation rate. Fig. 2 shows that we obtain asymptotic Kennicutt-Schmidt relations with slope $1.5\left(\dot{\rho}_{\mathrm{s}}=\rho \varepsilon_{\mathrm{ff}} / t_{\mathrm{ff}} \propto \rho^{1.5}\right)$ towards high densities, which is a consequence of the asymptotically constant star formation efficiency $\varepsilon_{\mathrm{ff}}$. Depending on the metallicity and other parameters, the threshold densities are typically between 20 and about $200 \mathrm{~cm}^{-3}$. For reasonable choices of the model coefficients that control internal turbulence driving and heating, a star formation efficiency of around 0.5 per cent is obtained above the threshold densities, in agreement with observed values (e.g. Krumholz \& Tan 2007; Bigiel et al. 2008 , 2011; Onodera et al. 2010; Schruba et al. 2011). External turbulence driving (i.e. energy transfer from larger scales via the turbulent cascade) decreases the star formation rate and slightly changes the slope of the power-law branches (Fig. 21, right-hand panel). This is primarily caused by the effect of the turbulent pressure on the average density of the cold phase, while the direct influence of turbulence on the star formation efficiency, following the prescription of PN11, plays a role in violently turbulent environments. In the latter case, the production of molecular hydrogen fraction is also affected via the turbulent clumping factor. Remarkably, the star formation efficiency is quite sensitive on the factor $b$ in equation (23) for the width of the density pdf as a function of the turbulent Mach number in the cold-gas phase. As shown by Federrath et al. (2010), $b$ is related to the mixture of the solenoidal and compressive components of the turbulent velocity field. Moreover, they concluded from comparisons with observed two-point statistics of turbulence in molecular clouds that this mixture varies for different clouds. Thus, it appears to be important to account for variations in the turbulence statistics.

Recent observations indicate a particularly tight correlation of the star formation rate with the molecular gas column densities in galaxies down to kiloparsec scales (KMT09 give an overview of observational results). Since we consider local regions of the ISM with a size smaller than the galactic disc thickness, it is not reasonable to express the results from our one-zone models in terms of column densities. For the same reason, comparisons with the model of Ostriker et al. (2010) are difficult. Nevertheless, we find that the relation between the equilibrium star formation rate and the density of molecular hydrogen closely follows a power law, particularly for solar metallicity. As one can see in the left-hand panel of Fig. 22 , the star formation rate is $\dot{\rho}_{\mathrm{s}} \propto \rho^{1.5}$ for sufficiently high density. Strong external turbulence forcing significantly reduces the star formation rate and the slope of the asymptote increases from 1.4 to about 1.6 (Fig. 22, right-hand panel). In this regard, it is interesting that KMT09 distinguish two different regimes, in which molecular clouds are either self-regulated (at low surface densities) or significantly affected by their galactic environment (at high surface densities). In the former case, they derive a slope of about 1.4, whereas the slope is about 1.6 in the latter case. A plausible interpretation in the context of our model is that these regimes roughly correspond to internal turbulence driving as the dominating production mechanism (negligible $\Sigma$ ) versus significant turbulence production by the transport from instabilities on large scale to molecular cloud scales (large $\Sigma$ ). To corroborate this interpretation, the model has to be applied in simulations of disc galaxies. The modelled equilibrium star formation rates and depletion time scales are roughly consistent with those found observationally (e.g. Schruba et al. 2011), and the modelled relation between star formation rate and molecular gas density is in agreement with a more or less constant molecular gas depletion time-scale as observed by Bigiel et al. (2011).
In such simulations as well as in cosmological simulations, it is common to assume a constant star formation efficiency beyond a certain threshold density. The results of our numerical study suggest that this is a reasonable approximation. However, rather than using this as an entirely phenomenological input to the simulations, the equilibrium values of the star formation efficiency calculated with our model follow from the sub-resolution physics of the ISM. Moreover, rather than setting stiff density thresholds, the model yields transition values depending on the varying gas density in numerical simulations. To utilize the equilibrium solutions as a parametrization of star formation, tables of the star formation efficiency as a function of density and metallicity can be calculated (the code calculating the efficiencies can be obtained from the authors upon request). The rate of external turbulent energy production could be estimated, for instance, from the large-scale velocity dispersion in galaxies (see Burkert et al. 2010).

While such a simplified approach has its merits, it cannot account for dynamical effects. A crucial problem is the calculation of the local rate of turbulent energy production on the grid scale due the shear of numerically resolved turbulent flow in a simulation (i.e. the energy transfer from length-scale greater than the size of the grid cells to unresolved length-scales). This is the meaning of $\Sigma$ if the proposed model is applied as an SGS model. Since the turbulent velocity fluctuations in the ISM can assume a significant fraction of the sound speed or even become supersonic, an incompressible turbulence model is not sufficient. SF11 provide a formula for $\Sigma$ in the highly compressible regime. A complete model for the turbulent multi-phase ISM and star formation is obtained by rewriting equations (42), (46), (61), (62), (73) and (75) as partial differential equations with fluid-dynamical advection terms, where the lengthscale $l$ is given by size of the grid cells, $\Delta$, and $e_{\mathrm{t}}=e_{\mathrm{sgs}}$ is identified with the unresolved fraction of the kinetic energy (see SF11). These equations supplement the Euler equations for the total gas density, the momentum and the total energy. Solving the complete set of equations will be a substantial numerical challenge.

To perform simulations of galaxies in cosmological environments, AMR is indispensable. Maier et al. (2009) incorporated an SGS turbulent energy equation for moderately compressible turbulence into AMR simulations of galaxy clusters. This method can also be applied using the multi-phase model for the turbulent ISM. Then, the length-scale $l$ of the model corresponds to the varying grid scale, and the scale-dependent turbulent energy has to be adjusted if refined grids are inserted or solutions on finer grids are projected to coarser grid levels.

An advantage of our model is that components can be modified, replaced and added as our understanding of the physics of the ISM progresses. For example, the computation of the dimensionless star formation rate from the turbulent cold-gas density pdf (see Section 3.1) is more or less heuristic. We anticipate that theoretical advances and results from small-scale simulations will soon lead to improvements. An important issue we have not considered so far is the influence of magnetic fields. The role of MHD turbulence is already emphasized by PN11. Although additional complications arise when magneto-turbulent fluctuations have to be treated on sub-grid scales, it is a problem that can be tackled. Furthermore, a more detailed treatment of chemical processes is desirable, although Krumholz \& Gnedin (2011) have demonstrated that the simple analytical model for the molecular hydrogen fraction in KMT09 agrees quite well with an explicit reaction network in cosmological simulations, at least if the metallicity is not much lower than solar. The multi-phase model has also to be adapted to the simulation framework. For cosmological simulations with relatively coarse 
resolutions, the neutral gas phases should be embedded in a hot ionized medium of low density. The other extreme are simulations of isolated disc galaxies with very high resolution, in which cold clumps can be marginally resolved so that several neighbouring grid cells are completely filled by cold gas. In this case, it is necessary to switch from the two-phase description to the one-phase limit.

The predictive power of astrophysical simulations, in which the ISM is only partially resolved, will increase either by applying the equilibrium solutions or by implementing the full multi-phase SGS model. This will allow us, in turn, to test and to modify the underlying physical assumptions.

\section{ACKNOWLEDGMENTS}

HB was financially supported by the DFG project Ni 516/701. WS is grateful for discussions with the participants of the 2010 summer programme 'Star Formation in Galaxies: From Recipes to Real Physics' at the Aspen Center for Physics, which helped to bring this work to maturity. We thank Jens Niemeyer for supporting this project and for many comments that helped to improve this paper. We also thank the referee for many helpful suggestions and Christoph Federrath for further comments.

\section{REFERENCES}

Agertz O., Lake G., Teyssier R., Moore B., Mayer L., Romeo A. B., 2009, MNRAS, 392, 294

Agertz O., Teyssier R., Moore B., 2011, MNRAS, 410, 1391

Alongi M., Bertelli G., Bressan A., Chiosi C., Fagotto F., Greggio L., Nasi E., 1993, A\&AS, 97, 851

Audit E., Hennebelle P., 2010, A\&A, 511, A76

Ballesteros-Paredes J., Vazquez-Semadeni E., Gazol A., Hartmann L. W., Heitsch F., Colin P., 2011, MNRAS, 416, 1436

Bertelli G., Bressan A., Chiosi C., Fagotto F., Nasi E., 1994, A\&AS, 106, 275

Bertoldi F., McKee C. F., 1992, ApJ, 395, 140

Bigiel F., Leroy A., Walter F., Brinks E., de Blok W. J. G., Madore B., Thornley M. D., 2008, AJ, 136, 2846

Bigiel F. et al., 2011, ApJ, 730, L13

Bonazzola S., Perault M., Puget J. L., Heyvaerts J., Falgarone E., Panis J. F., 1992, J. Fluid Mech., 245, 1

Bournaud F., Elmegreen B. G., Teyssier R., Block D. L., Puerari I., 2010, MNRAS, 409, 1088

Bressan A., Fagotto F., Bertelli G., Chiosi C., 1993, A\&AS, 100, 647

Burkert A. et al., 2010, ApJ, 725, 2324

Chabrier G., 2001, ApJ, 554, 1274

Chabrier G., Hennebelle P., 2010, ApJ, 725, L79

Cho W., Kim J., 2011, MNRAS, 410, L8

de Avillez M. A., Breitschwerdt D., 2004, A\&A, 425, 899

Dobbs C. L., Pringle J. E., 2010, MNRAS, 409, 396

Dobbs C. L., Glover S. C. O., Clark P. C., Klessen R. S., 2008, MNRAS, 389, 1097

Draine B. T., 1978, ApJS, 36, 595

Draine B. T., Bertoldi F., 1996, ApJ, 468, 269

Elmegreen B. G., Scalo J., 2004, ARA\&A, 42, 211

Federrath C., Glover S. C. O., Klessen R. S., Schmidt W., 2008, Phys. Scr. $\mathrm{T}, 132,014025$

Federrath C., Klessen R. S., Schmidt W., 2008, ApJ, 688, L79

Federrath C., Roman-Duval J., Klessen R. S., Schmidt W., Mac Low M., 2010, A\&A, 512, A81

Federrath C., Sur S., Schleicher D. R. G., Banerjee R., Klessen R. S., 2011, ApJ, 731, 62

Ferland G. J., Korista K. T., Verner D. A., Ferguson J. W., Kingdon J. B., Verner E. M., 1998, PASP, 110, 761
Gillmon K., Shull J. M., Tumlinson J., Danforth C., 2006, ApJ, 636, 891 Glover S. C. O., Clark P. C., 2012, MNRAS, 421, 9

Glover S. C. O., Mac Low M., 2007, ApJS, 169, 239

Gnedin N. Y., 1998, MNRAS, 294, 407

Gnedin N. Y., Kravtsov A. V., 2010, ApJ, 714, 287

Gnedin N. Y., Tassis K., Kravtsov A. V., 2009, ApJ, 697, 55 (GTK09)

Governato F., Willman B., Mayer L., Brooks A., Stinson G., Valenzuela O., Wadsley J., Quinn T., 2007, MNRAS, 374, 1479

Governato F. et al., 2010, Nat, 463, 203

Greif T. H., Glover S. C. O., Bromm V., Klessen R. S., 2010, ApJ, 716, 510 Hennebelle P., Chabrier G., 2008, ApJ, 684, 395

Hensler G., 2009, in Andersen J., Bland-Hawthorn J., Nordström B., eds,

IAU Symp. 254, Modelling the Disk (three-phase) Interstellar Medium.

Cambridge Univ. Press, Cambridge, p. 269

Hultman J., Pharasyn A., 1999, A\&A, 347, 769

Joung M. R., Mac Low M.-M., Bryan G. L., 2009, ApJ, 704, 137 (JMB09) Klessen R. S., 2001, ApJ, 556, 837

Klessen R. S., Hennebelle P., 2010, A\&A, 520, A17

Klypin A. A., 1998, in Mueller V., Gottloeber S., Muecket J. P., Wambsganss J., eds, Large Scale Structure: Tracks and Traces. World Scientific, Singapore, p. 47

Koppen J., Theis C., Hensler G., 1998, A\&A, 331, 524

Kotulla R., Fritze U., Weilbacher P., Anders P., 2009, MNRAS, 396, 462

Kravtsov A. V., 2003, ApJ, 590, L1

Kritsuk A. G., Norman M. L., Padoan P., Wagner R., 2007, ApJ, 665, 416

Kritsuk A. G., Norman M. L., Wagner R., 2011, ApJ, 727, L20

Krumholz M. R., Gnedin N. Y., 2011, ApJ, 729, 36

Krumholz M. R., McKee C. F., 2005, ApJ, 630, 250 (KM05)

Krumholz M. R., Tan J. C., 2007, ApJ, 654, 304

Krumholz M. R., McKee C. F., Tumlinson J., 2009, ApJ, 699, 850 (KMT09) Larson R. B., 1981, MNRAS, 194, 809

Lequeux J., 2005, The Interstellar Medium. Astronomy and Astrophysics Library, Springer, Berlin

Li Y., Mac Low M.-M., Klessen R. S., 2005, ApJ, 626, 823

Mac Low M.-M., Klessen R. S., 2004, Rev. Mod. Phys., 76, 125

Maier A., Iapichino L., Schmidt W., Niemeyer J. C., 2009, ApJ, 707, 40

Matzner C. D., McKee C. F., 2000, ApJ, 545, 364

Mayer L., Governato F., Kaufmann T., 2008, Adv. Sci. Lett., 1, 7

McKee C. F., Krumholz M. R., 2010, ApJ, 709, 308

McKee C. F., Ostriker J. P., 1977, ApJ, 218, 148

McKee C. F., Ostriker E. C., 2007, ARA\&A, 45, 565

Micic M., Glover S. C. O., Federrath C., Klessen R. S., 2012, MNRAS, doi:10.1111/j.1365-2966.2012.20477.x

Murante G., Monaco P., Giovalli M., Borgani S., Diaferio A., 2010, MNRAS, 405,1491

Murray N., 2011, ApJ, 729, 133

Onodera S. et al., 2010, ApJ, 722, L127

Ostriker E. C., McKee C. F., Leroy A. K., 2010, ApJ, 721, 975

Padoan P., Nordlund Å., 2011, ApJ, 730, 40 (PN11)

Rachford B. L. et al., 2002, ApJ, 577, 221

Rachford B. L. et al., 2009, ApJS, 180, 125

Raiteri C. M., Villata M., Navarro J. F., 1996, A\&A, 315, 105

Robertson B. E., Kravtsov A. V., 2008, ApJ, 680, 1083

Romeo A. B., Burkert A., Agertz O., 2010, MNRAS, 407, 1223

Scannapieco E., Brüggen M., 2010, MNRAS, 405, 1634 (SB10)

Schmidt W., Federrath C., 2011, A\&A, 528, A106 (SF11)

Schmidt W., Niemeyer J. C., Hillebrandt W., 2006, A\&A, 450, 265

Schmidt W., Federrath C., Hupp M., Kern S., Niemeyer J. C., 2009, A\&A, 494, 127

Schruba A., Leroy A. K., Walter F., Sandstrom K., Rosolowsky E., 2010, ApJ, 722, 1699

Schruba A. et al., 2011, AJ, 142, 37

Seifried D., Schmidt W., Niemeyer J. C., 2011, A\&A, 526, A14

Springel V., Hernquist L., 2003, MNRAS, 339, 289

Stinson G., Seth A., Katz N., Wadsley J., Governato F., Quinn T., 2006 , MNRAS, 373, 1074

Tasker E. J., Tan J. C., 2009, ApJ, 700, 358

Thornton K., Gaudlitz M., Janka H., Steinmetz M., 1998, ApJ, 500, 95 
Toomre A., 1964, ApJ, 139, 1217

Wada K., Norman C. A., 2007, ApJ, 660, 276

Wada K., Meurer G., Norman C. A., 2002, ApJ, 577, 197

Wang H.-H., Klessen R. S., Dullemond C. P., van den Bosch F. C., Fuch B., 2010, MNRAS, 407, 705

Wolfire M. G., Hollenbach D., McKee C. F., Tielens A. G. G. M., Bakes E. L. O., 1995, ApJ, 443, 152
Wolfire M. G., Tielens A. G. G. M., Hollenbach D., Kaufman M. J., 2008, ApJ, 680, 384

Yepes G., Kates R., Khokhlov A., Klypin A., 1997, MNRAS, 284, 235

This paper has been typeset from a $\mathrm{T}_{\mathrm{E}} \mathrm{X} / \mathrm{LT} \mathrm{T} \mathrm{X}$ file prepared by the author. 
A.2 Publikation Braun et al., 2014 


\title{
Large-eddy simulations of isolated disc galaxies with thermal and turbulent feedback
}

\author{
H. Braun, ${ }^{1 \star}$ W. Schmidt, ${ }^{1 \star}$ J. C. Niemeyer ${ }^{1}$ and A. S. Almgren ${ }^{2}$ \\ ${ }^{1}$ Institut für Astrophysik, Universität Göttingen, Friedrich-Hund Platz 1, D-37077 Göttingen, Germany \\ ${ }^{2}$ Center for Computational Sciences and Engineering, Lawrence Berkeley National Laboratory, Berkeley, CA 94720, USA
}

Accepted 2014 June 3. Received 2014 June 3; in original form 2013 December 18

\begin{abstract}
We present a subgrid-scale model for the Multi-phase Interstellar medium, Star formation, and Turbulence (MIST) and explore its behaviour in high-resolution large-eddy simulations of isolated disc galaxies. MIST follows the evolution of a clumpy cold and a diffuse warm component of the gas within a volume element which exchange mass and energy via various cooling, heating and mixing processes. The star formation rate is dynamically computed from the state of the gas in the cold phase. An important feature of MIST is the treatment of unresolved turbulence in the two phases and its interaction with star formation and feedback by supernovae. This makes MIST a particularly suitable model for the interstellar medium in galaxy simulations. We carried out a suite of simulations varying fundamental parameters of our feedback implementation. Several observational properties of galactic star formation are reproduced in our simulations, such as an average star formation efficiency $\sim 1$ per cent, a typical velocity dispersion around $\sim 10 \mathrm{~km} \mathrm{~s}^{-1}$ in star-forming regions, and an almost linear relationship between the column densities of star formation and dense molecular gas.
\end{abstract}

Key words: turbulence-methods: numerical-stars: formation-galaxies: ISM.

\section{INTRODUCTION}

Stars are a product of a complex sequence of competing and interacting processes on a vast range of spatial and temporal scales that concentrate initially dilute gas into compact cores. It is not yet fully understood how the interplay of all of the processes involved, such as gravitational collapse, cooling, turbulence, magnetism, and stellar feedback, leads to the observed properties of the interstellar medium and stars in galaxies. A recent review on the properties of star formation was presented by Kennicutt \& Evans (2012). Of particular interest is the mechanism regulating the observed low efficiency of star formation. Measures of the star formation efficiency are gas depletion - or consumption - time-scales $\tau_{\text {dep }}=M_{\text {gas }} / \dot{M}_{*}$, which relate star formation to the available gas supply. Wong \& Blitz (2002), Evans (2008), Bigiel et al. (2008), Blanc et al. (2009) infer $\tau_{\text {dep }} \sim 1-2$ Gyr in local disc galaxies from $\mathrm{CO}-\mathrm{H} \alpha$-, and UV-measurements with resolutions down to 200 pc. Comparable measurements of gas-rich galaxies by Daddi et al. (2010), Tacconi et al. (2013), Saintonge et al. (2013) and others indicate a significantly shorter time $\tau_{\text {dep }} \sim 0.5 \mathrm{Gyr}$, corresponding to a relative gas consumption rate per free-fall time $\epsilon_{\mathrm{ff}} \sim 0.01$. According to the KS relation (Schmidt 1959; Kennicutt 1998, and others), the star forma-

^E-mail: hbraun@astro.physik.uni-goettingen.de (HB); schmidt@astro. physik.uni-goettingen.de (WS) tion rate is well correlated with the local gas supply. The power-law slope of measured KS relations depends, however, on the tracers used, the resolution achieved, and other observational limitations (e.g. Lada, Lombardi \& Alves 2010; Onodera et al. 2010; Leroy et al. 2013). Recent observations show a good linear correlation between star formation rate and dense/molecular gas (Gao \& Solomon 2004; Lada et al. 2010; Bigiel et al. 2011). Evans et al. (2009) and Murray (2011) showed that the local efficiency $\epsilon_{\mathrm{ff}, \mathrm{MC}} \simeq 0.1$ in individual actively star-forming molecular clouds is much greater than the efficiency on galactic scales, and their depletion time-scale is considerably shorter ( $\tau_{\text {dep }}<100 \mathrm{Myr}$ ). This implies that molecular clouds convert a sizable fraction $0.1-0.4$ of their mass into stars during their lifetime (a few $10 \mathrm{Myr}$, see e.g. Blitz et al. 2007; McKee \& Ostriker 2007; Miura et al. 2012) before they are destroyed by supernova explosions ( $\mathrm{SNe}$ ) and stellar winds.

Actively star-forming molecular clouds are known to be strongly supersonically turbulent with typical velocity dispersions around $10 \mathrm{~km} \mathrm{~s}^{-1}$ (e.g. Leroy et al. 2008; Stilp et al. 2013), or larger in interacting galaxies (e.g. Herrera, Boulanger \& Nesvadba 2011). Regulation by supersonic turbulence is a good candidate to theoretically explain the observed properties of star formation, as it globally supports a molecular cloud against gravity, but locally produces over-dense filaments and knots that may collapse into stars. A variety of approaches have been developed in the past years to derive star formation efficiencies from statistical properties of gravo-turbulent fragmentation inside molecular clouds (Krumholz 


\section{H. Braun et al.}

\& McKee 2005; Hennebelle \& Chabrier 2011; Padoan \& Nordlund 2011, hereafter PN11; Padoan, Haugbølle \& Nordlund 2012; Federrath \& Klessen 2013, hereafter FK13). As supersonic turbulence decays on relatively short time-scales of the order of the sound crossing time, it has to be maintained by some production mechanism over the lifetime of a molecular cloud. Processes such as large-scale shear and instabilities in galactic discs (e.g. Gómez \& Cox 2002; Wada, Meurer \& Norman 2002; Kim, Ostriker \& Stone 2003; Kim \& Ostriker 2007; Agertz et al. 2009; Krumholz $\&$ Burkert 2010), accretion of gas on to a galaxy (e.g. Hopkins, Kereš \& Murray 2013; Genel, Dekel \& Cacciato 2012; Elmegreen \& Burkert 2010; Klessen \& Hennebelle 2010), and merger events or other galactic interactions (e.g. Teyssier, Chapon \& Bournaud 2010; Bournaud et al. 2011) come into question here, but also local processes like stellar winds (e.g. Vink, de Koter \& Lamers 2000; Wolf-Chase, Barsony \& O’Linger 2000; Vink 2011), radiation pressure (Krumholz \& Thompson 2012) and SNe (e.g. Vollmer \& Beckert 2003; Agertz et al. 2009; Ostriker \& Shetty 2011), or the effects of thermal instabilities (e.g. Wada \& Norman 2001; Kritsuk \& Norman 2002; Iwasaki \& Inutsuka 2014) are possible turbulence production mechanisms.

In order to numerically simulate a realistic galaxy as a whole, star formation and the entailing stellar feedback have to be taken into account. However, the resolution to properly follow the evolution inside star-forming clouds in a galactic scale simulation is far from being feasible with contemporary computational resources. Recent simulations of isolated disc galaxies (IDG) feature resolutions down to a few parsec or even a fraction of a parsec (e.g. Monaco et al. 2012; Benincasa et al. 2013; Booth et al. 2013; Dobbs \& Pringle 2013; Hopkins et al. 2013; Renaud et al. 2013), while simulations of galaxies from cosmological initial conditions reach resolutions of some 10 parsec (e.g. Agertz et al. 2009; Kraljic, Bournaud \& Martig 2012; Munshi et al. 2013). To tackle sub-resolution processes, an appropriate subgrid-scale (SGS) model has to be applied. In the last decade a wide range of different models have been devised to effectively describe star formation and stellar feedback (e.g. Stinson et al. 2006, 2013; Wise et al. 2012; Agertz et al. 2013) using resolved quantities and assumptions about the small-scale properties of the ISM.

In galaxy simulations, the star formation rate $\dot{\rho}_{s}$ is usually modelled using a constant efficiency $\epsilon_{\text {ff }}$ per free-fall time, which locally enforces a KS relation

$\dot{\rho}_{s}=\epsilon_{\mathrm{ff}} \frac{\rho}{\tau_{\mathrm{ff}}} \propto \rho^{1.5}$,

where $\rho$ is the local gas density and $\tau_{\mathrm{ff}} \propto \rho^{-0.5}$ the local free-fall time. To avoid spurious star formation, additional constraints are applied, for example, a threshold for the minimal density required for star formation and a maximal temperature. More sophisticated models distinguish between different gas components. Gnedin, Tassis \& Kravtsov (2009) suggest to relate the star formation rate to the density of molecular gas instead of the total gas density. Murante et al. (2010) use a simple multi-phase approach to determine the fraction of the gas density that is available for star formation. For the simulations presented in this article, we use a multi-phase model for the ISM, including an estimation of the amount of shielded molecular gas (Braun \& Schmidt 2012, hereafter BS12). The star formation efficiency in the molecular gas is dynamically computed from the numerically unresolved turbulence energy, which is determined by a SGS model for compressible turbulence (see Schmidt \& Federrath 2011, hereafter SF11). Since we incorporate the coupling between resolved and unresolved scales as turbulent stresses in the
Euler equations, our galaxy simulations are large-eddy simulations (LES). Moreover, we apply the energy-conserving AMR techniques presented in Schmidt et al. (2014). The diagonal part of the turbulent stresses acts as non-thermal pressure that usually dominates over the thermal pressure in cold and dense environments. This allows us to apply both thermal and turbulent feedback by channelling a fraction of the SN energy into the production of SGS turbulence energy. As we will show, this has important consequences for the regulation of star formation. In a way, this is similar to kinetic feedback (see e.g. Agertz et al. 2013), with the important difference that we assume that turbulent motions are mainly excited on length scales below the grid resolution. For the thermal feedback, a small portion of the $\mathrm{SNe}$ energy is stored in a non-cooling budget - decaying on a time-scale of $1 \mathrm{Myr}$ - to mimic the effect of hot SNe bubbles on sub-resolution scales, while the rest of the gas is allowed to cool radiatively. Although we include only effects of ionizing radiation from massive stars and SNe II, we are able to reproduce several observational features of star formation and turbulence in quiescent, gas-rich (or high redshift $z \sim 2$ ) disc galaxies and star-forming regions.

This paper is structured as follows. First we describe the numerical methods, the SGS model, and the setup of our IDG simulations in Sections 2 and 3. In Section 4, we present results from a suite of four simulations with different treatments of feedback, followed by our conclusions in Section 5 .

\section{NUMERICAL IMPLEMENTATION}

We carried out IDG simulations using the cosmological hydrodynamics code NYX (Almgren et al. 2013). NyX, built on the BoxLib software framework, uses Adaptive Mesh Refinement (AMR) to provide higher numerical resolution in sub-volumes of particular interest. NyX solves the standard Euler equations using an unsplit Piecewise Parabolic Method (PPM); additional source terms are treated via a predictor/corrector scheme. Nyx is capable of following the evolution of different collisionless massive particles in the $N$-body formalism using a Kick-Drift-Kick algorithm, and (self)gravity is taken into account using a Particle Mesh scheme with multigrid solver. We extended NYX to run adaptively refined LES of an IDG as described in the following.

To handle sub-resolution processes, such as star formation, stellar feedback, cooling, and thermal instability, we use a model based on BS12 with a few minor modifications, MIST (Multi-phase Interstellar medium, Star formation, and Turbulence model). The keyfeatures of MIST are the following.

(i) Atomic, metal line, and dust cooling, photoelectric heating on dust.

(ii) Separation of gas into two phases due to thermal instabilities that exchange energy and material via different mixing, heating, or cooling processes. The two phases represent a diffuse warm component and a clumpy, cold component of the gas. Balance of effective (i.e. thermal plus turbulent) pressure between the phases is assumed to obtain their respective densities.

(iii) Formation of stars from the molecular fraction of the cold phase that is shielded from dissociating radiation. The star formation rate is computed dynamically from the thermal and turbulent state of the gas.

(iv) Depending on the age of a stellar population the stellar feedback is applied. Lyman-continuum radiation and SNe II are taken into account. The SNe not only enrich the gas with metals, but also deposit kinetic energy and thermal energy into the gas. The $\mathrm{SNe}$ ejecta are treated as an additional sub-phase of the warm phase that 
does not cool efficiently and is gradually mixed with the rest of the warm phase.

(v) Unresolved turbulence is coupled to almost all processes implemented in MIST. Besides the source terms that are related to the scale separation for LES, small-scale pressure gradients caused by phase separation and $\mathrm{SNe}$ are taken into account.

An overview of important variables and coefficients is given in Table 1 .

\subsection{Gas dynamics}

As an extension to the standard compressible Euler equations we introduce a new degree of freedom in the form of the SGS turbulence energy density $\rho K$ (see SF11) and its source terms in order to model the behaviour of unresolved turbulent fluctuations. Furthermore we include the source terms as needed for the MIST model. The set of conservation equations for the evolution of gas becomes

$\frac{\partial \rho}{\partial t}+\nabla \cdot(\rho \boldsymbol{u})=-\dot{\rho}_{\mathrm{s}, \mathrm{SF}}+\dot{\rho}_{\mathrm{s}, \mathrm{FB}}$

$$
\begin{aligned}
& \frac{\partial(\rho \boldsymbol{u})}{\partial t}+\nabla \cdot\left[\rho \boldsymbol{u} \boldsymbol{u}+\left(p+\frac{2}{3} \rho K\right)-\tau^{*}\right] \\
& =\rho \boldsymbol{g}-\boldsymbol{u} \dot{\rho}_{\mathrm{S}, \mathrm{SF}}+\boldsymbol{u}_{\mathrm{s}, \mathrm{FB}} \dot{\rho}_{\mathrm{S}, \mathrm{FB}}, \\
& \frac{\partial(\rho E)}{\partial t}+\nabla \cdot\left[\rho \boldsymbol{u} E+\left(p+\frac{2}{3} \rho K\right) \boldsymbol{u}-\boldsymbol{u} \cdot \tau^{*}\right] \\
& \quad=\rho \boldsymbol{u} \cdot \boldsymbol{g}-\Lambda-\Pi_{\mathrm{TI}}-\Pi_{\mathrm{SGS}}-\left(E-e+e_{\mathrm{c}}\right) \dot{\rho}_{\mathrm{s}, \mathrm{SF}} \\
& \quad+\left(e_{\mathrm{SN}}+\frac{\boldsymbol{u}\left(2 \boldsymbol{u}_{\mathrm{s}, \mathrm{FB}}-\boldsymbol{u}\right)}{2}\right) \dot{\rho}_{\mathrm{s}, \mathrm{FB}}-\Pi_{\mathrm{SN}}, \\
& \frac{\partial(\rho K)}{\partial t}+\nabla \cdot\left(\rho \boldsymbol{u} K-\rho \kappa_{\mathrm{SGS}} \nabla K\right) \\
& \quad=\Pi_{\mathrm{SGS}}-\rho \varepsilon_{\mathrm{SGS}}+\Pi_{\mathrm{TI}}-K \dot{\rho}_{\mathrm{S}, \mathrm{SF}}+\Pi_{\mathrm{SN}}+\dot{\rho}_{\mathrm{s}, \mathrm{FB}}^{\mathrm{ex}}
\end{aligned}
$$

Here $\rho$ is the gas density, $\boldsymbol{u}$ the velocity vector, $E$ the total specific energy of the gas, $K$ the specific turbulent SGS-energy, $e$ the specific internal energy, $p=(\gamma-1) \rho e$ the thermal gas pressure (where $\gamma=5 / 3$ is the polytropic equation of state parameter), and $g$ the gravitational acceleration vector (see Section 2.1.1). The SGS turbulence model related quantities $\Pi_{\mathrm{SGS}}, \rho \varepsilon_{\mathrm{SGS}}$, and $\kappa_{\mathrm{SGS}}$ are defined in Section 2.1.2. For a definition of the star formation rate $\dot{\rho}_{\mathrm{s}, \mathrm{SF}}$ see Section 2.2.2. The stellar feedback rate $\dot{\rho}_{\mathrm{s}, \mathrm{FB}}$, the mass-weighted average velocity $\boldsymbol{u}_{\mathrm{S}, \mathrm{FB}}$ of SN-ejecta, and the specific supernova energy deposit $e_{\mathrm{SN}}=6.5 \times 10^{49} \mathrm{erg} / \mathrm{M}_{\odot}$ are explained in Section 2.3.2. We apply a multiphase model to determine the specific thermal energy $e_{\mathrm{c}}\left(T_{\mathrm{c}}=50 \mathrm{~K}\right)$ of the cold phase, and the net cooling rate $\Lambda$ (see Section 2.2.1) and the turbulence energy production via phase separation $\Pi_{\mathrm{TI}}$ and via $\mathrm{SN}$ feedback energy deposit $\Pi_{\mathrm{SN}}$ (see Section 2.2.2). To conserve the kinetic energy of SN-ejecta, $\dot{\rho}_{\mathrm{s}, \mathrm{FB}}^{\mathrm{ex}}$ is added to $\rho K$ (see equation 34 in Section 2.3.2).

\subsubsection{Gravity}

The massive components in our IDG simulations are dark matter, baryonic gas, and stars, where the dark matter component is assumed to be a static halo, and the other two are dynamically evolved. The gravitational acceleration vector $\boldsymbol{g}$ is computed as the sum of a static acceleration due to the dark matter halo and the negative gradient of the gravitational potential due to the dynamical components:

$\boldsymbol{g}=\boldsymbol{g}_{\mathrm{dm}}-\nabla \Phi_{\mathrm{dyn}}$,

with the static acceleration $\boldsymbol{g}_{\mathrm{dm}}$ (see equation 37 in Section 3.1). $\Phi_{\text {dyn }}$ represents the solution of Poisson's equation

$\Delta \Phi_{\mathrm{dyn}}=4 \pi G\left(\rho_{\mathrm{dyn}}-\bar{\rho}_{\mathrm{dyn}}\right)$,

where $\rho_{\text {dyn }}=\rho+\rho_{\mathrm{s}}, \bar{\rho}_{\text {dyn }}$ is the mean of $\rho_{\text {dyn }}, \rho_{\mathrm{s}}$ is the total stellar density, and $G$ is the gravitational constant.

\subsubsection{Hydrodynamical turbulence model}

The interaction between resolved and unresolved turbulent velocity fluctuations is modelled using the SGS turbulence stress tensor $\tau$, which can be seen as an analogue to the viscous dissipation tensor in the Navier-Stokes equations. With $u_{i, k}:=\partial u_{i} / \partial x_{k}$ its components following SF11 read

$$
\begin{aligned}
\tau_{i j}= & 2 C_{\tau 1} \Delta(2 \rho K)^{1 / 2} S_{i j}^{*}-2 C_{\tau 2} \rho K \frac{u_{i, k} u_{j, k}}{u_{l, m} u_{l, m}} \\
& -\frac{2}{3}\left(1-C_{\tau 2}\right) \rho K \delta_{i j},
\end{aligned}
$$

where

$S_{i j}^{*}=S_{i j}-\frac{1}{3} \delta_{i j} d=\frac{1}{2}\left(u_{i, j}+u_{j, i}\right)-\frac{1}{3} \delta_{i j} u_{k, k}$

is the trace-free rate of strain, and $\Delta$ the grid scale. The tracefree stress tensor $\tau^{*}$, used in equations (3) and (4), is given by $\tau_{\mathrm{ij}}^{*}=\tau_{\mathrm{ij}}-2 \delta_{\mathrm{ij}} K / 3$. The SGS turbulence energy production rate $\Pi_{\mathrm{SGS}}$, the SGS turbulence dissipation rate $\rho \varepsilon_{\mathrm{SGS}}$ (which does not appear in equation 4 as it is absorbed into $\Lambda$ ), and the SGS turbulence diffusivity $\kappa_{\mathrm{SGS}}$ are given by

$\Pi_{\mathrm{SGS}}=\tau_{i j} S_{i j}$,

$\left(\rho \varepsilon_{\mathrm{SGS}}\right)=\frac{\rho C_{\varepsilon} K^{3 / 2}}{\Delta}$,

$\kappa_{\mathrm{SGS}}=C_{\kappa}(2 K)^{1 / 2}$.

We use the closure coefficients $C_{\tau 1}=0.02, C_{\tau 2}=0.75, C_{\varepsilon}=1.58$, and $C_{K}=0.65$ as determined by SF11 for compressible turbulence.

\subsection{Non-adiabatic physics}

In the following we describe how unresolved physics such as heating, cooling, star formation and stellar feedback was implemented in the code NyX. For a more detailed description of the underlying model we refer to BS12. As input for the computation of the nonadiabatic physics sources we need the hydrodynamical state, the source terms belonging only to the SGS turbulence model and the stellar feedback terms. Contrary to BS12, stellar feedback is considered an external source in the calculation, as it depends on the stellar population represented by $N$-body particles but not on the hydrodynamical state. Given the SGS- and stellar feedback source terms, the actual sources are calculated by subcycling the BS12 model ODEs locally in a grid cell, to resolve all time-scales of relevant processes, particularly the cooling time-scale, and then averaging the rate of change over the hydro-step. To follow the metal enrichment, we 


\section{H. Braun et al.}

Table 1. Important variables and coefficients.

\begin{tabular}{|c|c|c|c|}
\hline Symbol & Value & Description & Reference \\
\hline \multicolumn{4}{|c|}{ Hydrodynamics: } \\
\hline$\rho$ & & Total gas density & Equation (2) \\
\hline$\rho \boldsymbol{u}$ & & Vector of linear momentum density of gas & Equation (3) \\
\hline$\rho E$ & & Thermal plus (resolved) kinetic energy density of gas & Equation (4) \\
\hline$\rho K$ & & SGS turbulence energy density & Equation (5) \\
\hline$\rho_{\mathrm{c}}$ & & Fractional density of cold phase gas & BS12, equation (14) \\
\hline$\rho_{\mathrm{h}}$ & & Fractional density of hot SNe ejecta & Equation (35) \\
\hline$\rho_{\mathrm{H}}$ & & Hydrogen density & Equation (13) \\
\hline$\rho_{\mathrm{He}}$ & & Helium density & Equation (13) \\
\hline$\rho_{\mathrm{Z}}$ & & Metal density & Equation (13) \\
\hline$\gamma$ & $5 / 3$ & Polytropic index of equation of state & \\
\hline \multicolumn{4}{|c|}{ SGS turbulence: } \\
\hline$\Pi_{\text {SGS }}$ & & Production rate of SGS energy by turbulent stresses & SF11, equation (10) \\
\hline$\tau$ & & Turbulent stress tensor & SF11, equation (8) \\
\hline$C_{\tau 1}$ & 0.02 & Linear closure coefficient of turb. stresses & SF11 \\
\hline$C_{\tau 2}$ & 0.75 & Non-linear closure coefficient of turb. stresses & SF11 \\
\hline$C_{\varepsilon}$ & 1.58 & Dissipation coefficient of SGS turb. energy & SF11 \\
\hline$C_{\kappa}$ & 0.65 & Diffusion coefficient of SGS turb. energy & SF11 \\
\hline \multicolumn{4}{|c|}{ MIST: } \\
\hline$\rho_{\mathrm{c}, \mathrm{pa}}$ & & Average density of cold phase gas & BS12 \\
\hline$\rho_{\mathrm{w}, \mathrm{pa}}$ & & Average density of warm phase gas & BS12 \\
\hline$e_{\mathrm{w}}$ & & Specific thermal energy of warm gas & BS12 \\
\hline$\ell_{\mathrm{c}}$ & & Length scale of cold phase clumps & BS12 \\
\hline$\dot{\rho}_{\mathrm{s}, \mathrm{SF}}$ & & Star formation density & BS12, equation (17) \\
\hline$\dot{\rho}_{\mathrm{s}, \mathrm{FB}}$ & & SNe feedback density & BS12, Section 2.3.2 \\
\hline$\epsilon_{\mathrm{PN}}$ & & Star formation free-fall time efficiency in cold gas & PN11, FK13, equation (19) \\
\hline$\Pi_{\mathrm{SN}}$ & & Production rate of SGS energy via SNe feedback & Equation (22) \\
\hline$\Pi_{\mathrm{TI}}$ & & Production rate of SGS energy via phase separation & Equation (21) \\
\hline$A_{\mathrm{SN}}$ & & SNe evaporation coefficient & BS12, equation (15) \\
\hline$f_{\mathrm{TI}}$ & 0 or 1 & Thermal instability switch & BS12 \\
\hline$\epsilon_{\mathrm{TI}}$ & 0.025 & Efficiency of SGS energy production by phase separation & BS12 \\
\hline$\epsilon_{\mathrm{SN}}$ & 0.085 & Efficiency of SGS energy production by SNe feedback & BS12 \\
\hline$e_{\mathrm{SN}}$ & $6 \times 10^{49} \mathrm{erg} / \mathrm{M}_{\odot}$ & Energy release per $\mathrm{M}_{\odot}$ of $\mathrm{SNe}$ II & BS12 \\
\hline$\tau_{\mathrm{h}}$ & $1 \mathrm{Myr}$ & Decay parameter of the hot $\mathrm{SNe}$ gas & Section 2.3 .2 \\
\hline$e_{\mathrm{h}}$ & $0.1 \times e_{\mathrm{SN}}$ & Specific thermal energy of gas in SNe bubbles & Section 2.3 .2 \\
\hline$e_{\mathrm{c}}$ & $e\left(T_{\mathrm{c}} \equiv 50 \mathrm{~K}\right)$ & Specific thermal energy of cold gas & $\mathrm{BS} 12$ \\
\hline$f_{\text {loss }}$ & 0.4 & Fraction of prestellar mass loss & BS12 \\
\hline$b$ & $1 / 3 \ldots 1$ & Compressive factor, density PDF broadening parameter & BS12, equation (23) \\
\hline$\eta$ & $1 / 3$ & Turbulent velocity scaling coefficient of warm gas & BS12 \\
\hline$\zeta$ & 0.1 & Metal loading fraction of SNe ejecta & BS12 \\
\hline
\end{tabular}

Abbreviated references: BS12 - Braun \& Schmidt (2012), SF11 - Schmidt \& Federrath (2011), FK13 - Federrath \& Klessen (2013), PN11 - Padoan \& Nordlund (2011).

calculate three hydrogen density $\rho_{\mathrm{H}}$, helium density $\rho_{\mathrm{He}}$ and metal density $\rho_{\mathrm{Z}}$. Their conservation equations are of the form

$\frac{\partial \rho_{\mathrm{X}}}{\partial t}+\nabla \cdot\left(\rho_{\mathrm{X}} \boldsymbol{u}\right)=-\frac{\rho_{\mathrm{X}}}{\rho} \dot{\rho}_{\mathrm{s}, \mathrm{SF}}-\left.\frac{\partial \rho_{\mathrm{s}, \mathrm{X}}}{\partial t}\right|_{\mathrm{FB}}$,

where $X$ indicates one of the species $\mathrm{H}, \mathrm{He}$, or $\mathrm{Z}$, and $\partial \rho_{\mathrm{s}, \mathrm{X}} /\left.\partial t\right|_{\mathrm{FB}}=$ : $\dot{\rho}_{X s, F B}$ is the ejection rate of that species by SNe (see Section 2.3.2).

\subsubsection{Cold and warm gas phases}

To keep track of the multiphase state in a grid cell, we introduce an additional passively advected quantity, the cold-phase fractional density $\rho_{\mathrm{c}}$, from which we can easily reconstruct the warm-phase density $\rho_{\mathrm{w}}=\rho-\rho_{\mathrm{c}}$. The warm phase thermal energy is given by $\rho_{\mathrm{w}} e_{\mathrm{w}}=\rho e-\rho_{\mathrm{c}} e_{\mathrm{c}}$ with a constant specific thermal energy $e_{\mathrm{c}}$ of the cold phase, corresponding to a temperature $T_{\mathrm{c}}=50 \mathrm{~K}$. The conservation equation of $\rho_{\mathrm{c}}$ reads

$$
\begin{aligned}
\frac{\partial \rho_{\mathrm{c}}}{\partial t}+\nabla \cdot\left(\rho_{\mathrm{c}} \boldsymbol{u}\right)= & \frac{\Lambda_{\mathrm{c}}+\Lambda_{\mathrm{w}} f_{\mathrm{TI}}}{e_{\mathrm{w}}-e_{\mathrm{c}}} \\
& -\dot{\rho}_{\mathrm{s}, \mathrm{SF}}-A_{\mathrm{SN}} \dot{\rho}_{\mathrm{s}, \mathrm{FB}},
\end{aligned}
$$

where

$$
\begin{aligned}
& \Lambda_{\mathrm{c}}=-\rho_{\mathrm{c}} \varepsilon_{\mathrm{SGS}}-\Gamma_{\mathrm{c}}^{\mathrm{PAH}}-\frac{\rho_{\mathrm{c}}}{\rho} \Gamma^{\mathrm{Lyc}} \text { and } \\
& \Lambda_{\mathrm{w}}=\Lambda_{\mathrm{w}}^{\mathrm{rad}}-\rho_{\mathrm{w}} \varepsilon_{\mathrm{SGS}}-\Gamma_{\mathrm{w}}^{\mathrm{PAH}}-\frac{\rho_{\mathrm{w}}}{\rho} \Gamma^{\mathrm{Lyc}}
\end{aligned}
$$

are the net cooling rates of the cool and warm phase, respectively. $\Lambda_{\mathrm{c}}$ is effectively a heating rate. Material is removed from the cold phase and transferred to the warm phase instead of increasing $u_{\mathrm{c}} \equiv$ const.

$A_{\mathrm{SN}}=13826\left(\frac{\rho_{\mathrm{w}, \mathrm{pa}}}{\frac{\mathrm{m}_{\mathrm{H}}}{\mathrm{cm}^{3}}}\right)^{\frac{4}{5}}\left(\frac{\ell_{\mathrm{c}}}{\mathrm{pc}}\right)^{-\frac{6}{5}}\left(\frac{\rho_{\mathrm{c}}}{\rho_{\mathrm{c}, \mathrm{pa}}}\right)^{\frac{3}{5}}$

MNRAS 442, 3407-3426 (2014) 
is the $\mathrm{SN}$ cold-phase evaporation coefficient, and $\epsilon_{\mathrm{TI}}=0.025$ is the efficiency parameter for turbulence production by the thermal instability, if the indicator $f_{\mathrm{TI}}=1$ (see BS12). Here $\Gamma^{\mathrm{PAH}}$ is the photoelectric heating rate, $\Gamma^{\mathrm{Lyc}}$ the heating rate due to Lyman continuum radiation from young, massive stars (see Section 2.3.2), and

$\Lambda_{\mathrm{w}}^{\mathrm{rad}}=\frac{\hat{\rho}_{\mathrm{w}}}{\rho_{\mathrm{w}, \mathrm{pa}}} \hat{\Lambda}_{\mathrm{w}}^{\mathrm{rad}}\left(\rho_{\mathrm{w}, \mathrm{pa}}, Z, \hat{T}_{\mathrm{w}}\right)$

the radiative cooling rate, which is interpolated from a cooling table. These tabled cooling rates were computed using the photoionization program package CLOUDY (Ferland et al. 1998, version 08.00). $\rho_{\mathrm{w}, \mathrm{pa}}$ and $\rho_{\mathrm{c}, \mathrm{pa}}$ are the average densities of the warm and the cold phase, respectively. Those are computed from the fractional phase densities $\left(\rho_{\mathrm{c}}\right.$ and $\left.\rho_{\mathrm{w}}\right)$ and the thermal and turbulent energies $\left(e_{\mathrm{c}}, e_{\mathrm{w}}\right.$, and $\left.K\right)$ by assuming balance of effective (thermal plus turbulent) pressure between the phases at cold clump scale $\ell_{\mathrm{c}}$, as explained in detail in BS12. $Z$ is the metallicity of the gas. $\hat{T}_{\mathrm{w}}=$ $\hat{T}_{\mathrm{w}}\left(\hat{e}_{\mathrm{w}}, Z\right)$ and $\hat{\rho}_{\mathrm{w}}$ are the temperature and the fractional density of the warm-phase gas, that is allowed to cool radiatively. Note that $\hat{T}_{\mathrm{w}}$ and $\hat{\rho}_{\mathrm{w}}$ may differ from $T_{\mathrm{w}}$ and $\rho_{\mathrm{w}}$ in areas affected by recent $\mathrm{SNe}$ feedback. The treatment of the third gaseous phase, the hot $\mathrm{SNe}$ ejecta, which is prevented from cooling, is described in Section 2.3.2. The total net cooling rate, used in equation (4), is then given by $\Lambda=\Lambda_{\mathrm{c}}+\Lambda_{\mathrm{w}}$.

\subsubsection{Star formation rate}

Stars are assumed to form from the molecular fraction of the gas in the cold phase, $f_{\mathrm{H}_{2}} \rho_{\mathrm{c}}$ at a rate (Krumholz, McKee \& Tumlinson 2009)

$\dot{\rho}_{\mathrm{s}, \mathrm{SF}}=\frac{f_{\mathrm{H}_{2}} \rho_{\mathrm{c}} \epsilon_{\mathrm{PN}}}{t_{\mathrm{c}, \mathrm{ff}}}$,

where $\epsilon_{\mathrm{PN}}$ is the formation rate of gravitationally bound cores per free-fall time

$t_{\mathrm{c}, \mathrm{ff}}=\sqrt{\frac{3 \pi}{32 G \rho_{\mathrm{c}, \mathrm{pa}}}}$.

To calculate $\epsilon_{\mathrm{PN}}$, we use the PN11 model in the single free-fall formulation of FK13

$\epsilon_{\mathrm{PN}}=\frac{\left(1-f_{\text {loss }}\right) r_{\text {crit }}^{\frac{1}{2}}}{2}\left(1+\operatorname{erf}\left[\frac{\sigma_{\mathrm{c}}^{2}-2 \log \left(r_{\text {crit }}\right)}{\left(8 \sigma_{\mathrm{c}}^{2}\right)^{\frac{1}{2}}}\right]\right)$.

Here $f_{\text {loss }}=0.4$ is the fraction of mass in gravitationally bound cores lost during prestellar collapse through winds etc., and $\sigma_{\mathrm{c}}=$ $\sqrt{\log \left(1+b^{2} \mathcal{M}_{\mathrm{c}}^{2}\right)}$ the standard deviation of the assumed density probability density function (PDF) of log-normal shape. The broadening parameter $b$ is set depending on which of the three production terms of turbulence energy in equation (5) is locally the dominant one

$\Pi_{\max }=\max \left[\Pi_{\mathrm{SN}}, \Pi_{\mathrm{TI}}, \Pi_{\mathrm{SGS}}\right]$,

where

$\Pi_{\mathrm{TI}}=\Lambda_{\mathrm{W}} f_{\mathrm{TI}} \epsilon_{\mathrm{TI}}$

is the contribution due to thermal instability, and

$\Pi_{\mathrm{SN}}=\dot{\rho}_{\mathrm{S}, \mathrm{FB}} e_{\mathrm{SN}} \epsilon_{\mathrm{SN}}$ describes turbulence production by SNe. $\epsilon_{\mathrm{SN}}=0.085$ is the fraction of the energy released by SN that is deposited in the form of turbulent energy. We define $b$ by

$b= \begin{cases}1 / 3 & \text { if } \Pi_{\mathrm{SGS}}=\Pi_{\max } \\ 2 / 3 & \text { if } \Pi_{\mathrm{TI}}=\Pi_{\max } \\ 1 & \text { if } \Pi_{\mathrm{SN}}=\Pi_{\max }\end{cases}$

Here we assume the large-scale driving $\Pi_{\mathrm{SGS}}$ to be mostly caused by shear, the SNe driving to be mostly compressive, and the thermal instability driving to be of intermediate type. The corresponding values follow from Federrath et al. (2010).

To obtain the turbulent Mach-number $\mathcal{M}_{\mathrm{c}}$ of the cold phase, the SGS energy $K$ has to be rescaled from the grid scale $\Delta$ to the cold clump scale $\ell_{\mathrm{c}}$, assuming a Kolmogorov velocity scaling exponent $\eta=1 / 3: 1$

$\mathcal{M}_{\mathrm{c}}^{2}=\frac{2 K\left(\frac{\ell_{\mathrm{c}}}{\Delta}\right)^{2 \eta}}{\gamma(\gamma-1) e_{\mathrm{c}}}$.

The critical over-density ratio $r_{\text {crit }}=\rho_{\text {crit }} / \rho_{\mathrm{c} \text {, pa }}$, above which a bound object is formed, is given by (FK13)

$r_{\text {crit }}=0.0067 \times \frac{5 \times 2 K\left(\frac{\ell_{\mathrm{c}}}{\Delta}\right)^{2 \eta}}{\pi G \rho_{\mathrm{c}, \mathrm{pa}} \ell_{\mathrm{c}}^{2}} \mathcal{M}_{\mathrm{c}}^{2}$.

The molecular fraction of cold gas $f_{\mathrm{H}_{2}}$ is computed from the cold and warm phase fractional densities and energies, assuming effective pressure equilibrium, using a Stömgren-like approach. The penetration depth of impinging radiation into a spherical cold clump of diameter $\ell_{\mathrm{c}}$ is determined by the balance between $\mathrm{H}_{2}$ production and dissociation due to $\mathrm{UV}$-photons. The dissociating radiation field $I_{v}$ is assumed to be homogenous and isotropic. However, in dense, cold environments, which are identified by $\rho_{\mathrm{c}, \mathrm{pa}}>10 \times \mu m_{\mathrm{H}} \mathrm{cm}^{-3}$ and $T(e, Z)<1000 \mathrm{~K}, I_{v}$ is dimmed by a factor

$f_{I_{v}}=\frac{1}{3}+\frac{2}{3} \max \left[1+\frac{T_{I_{v}}-T(e, Z)}{T_{I_{v}}}, 0\right]$,

with $T_{I_{v}}=500 \mathrm{~K}$, because of the assumed shielding from radiation by the environment.

The assumption of a log-normal shaped density PDF, which is an essential part of the theory of PN11, applies to turbulence in isothermal gas. For a consistent definition of the internal energy of the cold phase in MIST, an adiabatic exponent $\gamma=5 / 3$ is required. However, we assume a constant average temperature of the cold phase because of processes which are not explicitly treated. Both observational and numerical studies on the density PDFs show that the density PDF of the cold phase of the ISM is well approximated by a log-normal PDF (e.g. Hughes et al. 2013; Schneider et al. 2014). Although a power-law tail is generally found for actively star-forming clouds in which dense cores undergo gravitational collapse, FK13 point out that this does not significantly affect the star formation efficiency because the log-normal turbulent density fluctuations feed the collapsing gas that populates the power-law tail at high densities. Despite of the underlying inconsistency, all currently available analytic models for the calculation of star formation efficiencies, including PN11, are based on this assumption.

${ }^{1}$ We assume $\ell_{\mathrm{c}}$ to be the largest scale represented in the cold phase. Consequently, the scaling of the turbulent velocities is applied to those scales on which only the warm phase exists. Turbulence in the warm gas is usually subsonic or transonic at most. In this regime the assumption of a Kolmogorovtype scaling behaviour with coefficient $\eta=1 / 3$ seems valid. 


\section{H. Braun et al.}

Substituting PDFs with power-law tails into these models does not amend the problem because this would lead to divergent integrals. As a consequence, the construction of consistent models of the star formation efficiency is an open problem.

\subsection{Implementation of stellar particles}

\subsubsection{Stellar particle creation}

A particle is characterized by its position $\boldsymbol{x}_{\mathrm{p}}$, mass $m_{\mathrm{p}}$, velocity $\boldsymbol{u}_{\mathrm{p}}$, and an arbitrary number of additional properties. To handle the dynamical evolution of stars, we implemented a particle type with three additional properties: the initial mass $m_{\mathrm{pi}}$, creation time $t_{\mathrm{pc}}$, and metallicity $Z_{\mathrm{p}}$, which are needed for the application of stellar feedback. A stellar particle does not represent a single star, but a single stellar population with a normalized initial mass function (IMF) $\mathrm{d} N_{*} / \mathrm{d} m_{*}$ (where $N_{*}$ is the number of stars of individual initial mass $m_{*}$ per solar mass of stellar population) scaled by $m_{\mathrm{pi}}$. We use the IMF of Chabrier (2001).

To avoid the repeated creation of particles in all cells where $\operatorname{dot} \rho_{\mathrm{S}, \mathrm{SF}}>0$, we introduce a stellar density field $\rho_{\mathrm{s}, \mathrm{m}}$ that acts as an intermediate buffer for the stellar mass. This is treated as a passively advected quantity with respect to the hydro-solver, but massive with respect to gravity. Its conservation equation reads

$\frac{\partial \rho_{\mathrm{s}, \mathrm{m}}}{\partial t}+\nabla \cdot\left(\rho_{\mathrm{s}, \mathrm{m}} \boldsymbol{u}\right)=\dot{\rho}_{\mathrm{s}, \mathrm{SF}}-\left.\frac{\partial \rho_{\mathrm{s}, \mathrm{m}}}{\partial t}\right|_{\mathrm{SP}}$,

where $\partial \rho_{\mathrm{s}, \mathrm{m}} /\left.\partial t\right|_{\mathrm{SP}}$ represents the mass transfer from $\rho_{\mathrm{s}, \mathrm{m}}$ into stellar particles. The total stellar mass density is given by $\rho_{\mathrm{s}}=\rho_{\mathrm{s}, \mathrm{p}}+\rho_{\mathrm{s}, \mathrm{m}}$.

A pair of stellar particles $p^{1}$ and $p^{2}$ is created in the cell centre, if the agglomerated $\rho_{\mathrm{s}, \mathrm{m}}$ exceeds the threshold $\rho_{\mathrm{s}, \mathrm{m}, \max } \propto \Delta^{-2}$ (corresponding to a minimum particle pair mass $2 m_{\mathrm{p}, \min }=\rho_{\mathrm{s}, \mathrm{m}, \max } \Delta^{-3}$ $\approx 40 \mathrm{M}_{\odot}$ for a cell size of $\Delta \approx 30 \mathrm{pc}$ ), the mass is removed from $\rho_{\mathrm{s}, \mathrm{m}}$. The properties of the new particles are

$m_{\mathrm{p}}^{1,2}=\rho_{\mathrm{s}, \mathrm{m}} \Delta^{3} / 2$,

$\boldsymbol{u}_{\mathrm{p}}^{1,2}=\boldsymbol{u} \pm \boldsymbol{u}_{\mathrm{rnd}}$,

$m_{\mathrm{pi}}^{1,2}=\rho_{\mathrm{s}, \mathrm{m}} \Delta^{3} / 2$,

$t_{\mathrm{pc}}^{1,2}=t+\mathrm{d} t / 2$

$Z_{\mathrm{p}}^{1,2}=\rho_{\mathrm{Z}} / \rho$.

Here $\mathrm{d} t$ is the hydro time-step, and $\boldsymbol{u}_{\text {rnd }}$ is a random velocity (in opposite directions for $p^{1}$ and $p^{2}$ to conserve total momentum). This random velocity component is intended to reflect the unresolved motions of the cold clumps, which the stars originate from. Its absolute value is drawn from a Gaussian distribution with expectation value 0 and variance $\sigma_{u_{\mathrm{rnd}}}^{2}$

$\sigma_{u_{\mathrm{rnd}}}^{2}=f_{u_{\mathrm{rnd}}} 2 K\left(1-\left(\frac{\ell_{\mathrm{c}}}{\Delta}\right)^{2 \eta}\right)$.

The fudge factor $f_{u_{\mathrm{rnd}}}=\left(\left\langle m_{\mathrm{p}}\right\rangle m_{\mathrm{p}, \mathrm{th}}\right) / m_{\mathrm{p}}^{2}$ is designed to obtain $f_{u_{\text {rnd }}}^{-2} \boldsymbol{u}_{\text {rnd }}$ as random velocity component at the end of the particle growth phase, when the final $m_{\mathrm{pi}} \approx\left(\left\langle m_{\mathrm{p}}\right\rangle m_{\mathrm{p}, \text { th }}\right)^{1 / 2}$ is reached. $\left\langle m_{\mathrm{p}}\right\rangle$ is the mean mass of all stellar particles and $m_{\mathrm{p}, \text { th }}$ the upper threshold mass for particle growth.

A newly created stellar particle $p$ collects the stellar mass in $\rho_{\mathrm{s}, \mathrm{m}}$ along its path [using a Nearest Grid Point algorithm (NGP)]. Its properties are updated using

$$
\begin{aligned}
\dot{m}_{\mathrm{p}} & =m_{\mathrm{p}}+\rho_{\mathrm{s}, \mathrm{m}} \Delta^{3}, \\
\dot{\boldsymbol{u}}_{\mathrm{p}} & =\frac{\boldsymbol{u}_{\mathrm{p}} m_{\mathrm{p}}+\boldsymbol{u} \rho_{\mathrm{s}, \mathrm{m}} \Delta^{3}}{\dot{m}_{\mathrm{p}}}, \\
\dot{m}_{\mathrm{pi}} & =m_{\mathrm{pi}}+\rho_{\mathrm{s}, \mathrm{m}} \Delta^{3}, \\
\dot{t}_{\mathrm{pc}} & =\frac{t_{\mathrm{pc}} m_{\mathrm{p}}+(t+\mathrm{d} t / 2) \rho_{\mathrm{s}, \mathrm{m}} \Delta^{3}}{\dot{m}_{\mathrm{p}}}, \\
\dot{Z}_{\mathrm{p}} & =\frac{Z_{\mathrm{p}} m_{\mathrm{p}}+\frac{\rho \mathrm{Z}}{\rho} \rho_{\mathrm{s}, \mathrm{m}} \Delta^{3}}{\dot{m}_{\mathrm{p}}}, \\
\dot{\boldsymbol{x}}_{\mathrm{p}} & =\frac{\boldsymbol{x}_{\mathrm{p}} m_{\mathrm{p}}+\boldsymbol{x} \rho_{\mathrm{s}, \mathrm{m}} \Delta^{3}}{\dot{m}_{\mathrm{p}}} .
\end{aligned}
$$

The final mass is reached, when either $m_{\mathrm{p}}>m_{\mathrm{p} \text {, th }} \simeq 20 \times m_{\mathrm{p} \text {, min }}$ or $\left(t-t_{\mathrm{pc}}\right)>2 \mathrm{Myr}$.

\subsubsection{Feedback mechanism and hot phase}

We consider the two physical stellar feedback processes, Lyman continuum heating and $\mathrm{SNe}$ explosions, and use the equations in BS12 to compute their contributions to the source terms for the update of the hydrodynamical state.

The stellar mass sink field $\rho_{\mathrm{s}, \mathrm{m}}$ acts on the gas only via Lyman continuum heating $\Gamma^{\mathrm{Lyc}, \mathrm{m}}$, where we assume zero age of the stellar population it represents. The amount of feedback (the mass of $\mathrm{SNe}$ ejecta $m_{\mathrm{p}, \text { fb }}$, the mass in the different chemical species $m_{\mathrm{p}, \mathrm{X}}$, fb , and the heating rate due to Lyman continuum radiation $\Gamma^{\mathrm{Lyc}, \mathrm{p}}$ ) during a hydro time-step $\mathrm{d} t$ is computed individually for every stellar particle according to its properties. To obtain $\dot{\rho}_{\mathrm{s}, \mathrm{FB}}$, the feedback is mapped to the hydro-mesh using a cloud in cell (CIC) scheme and timeaveraged over the time-step $\mathrm{d} t$. The $\mathrm{SNe}$ ejecta mass is computed by

$m_{\mathrm{p}, \mathrm{fb}}=m_{\mathrm{pi}} \int_{t-t_{\mathrm{pc}}}^{t-t_{\mathrm{pc}}+\mathrm{d} t} m_{*} \frac{\mathrm{d} N_{*}}{\mathrm{~d} m_{*}} \frac{\mathrm{d} m_{*}}{\mathrm{~d} t^{\prime}} \mathrm{d} t^{\prime}$,

where $m_{*}=m_{*}\left(Z_{\mathrm{p}}, t^{\prime}\right)$ is the initial mass of an individual star that goes SN at an age of $t^{\prime}$ (Raiteri, Villata \& Navarro 1996). $m_{\mathrm{p}, \mathrm{fb}}$ is removed from the particle mass: $m_{\mathrm{p}}=m_{\mathrm{p}}-m_{\mathrm{p}, \mathrm{fb}}$. The metal load of the ejecta is given by $m_{\mathrm{p}, \mathrm{Z}, \mathrm{fb}}=m_{\mathrm{p}, \mathrm{fb}}\left(Z_{\mathrm{p}}+\zeta\right)$, where $\zeta$ is the fraction of metals produced in the dying stars, the other species scale linearly with metallicity. The total Lyc heating rate is $\Gamma^{\mathrm{Lyc}}=\Gamma^{\mathrm{Lyc}, \mathrm{m}}+\Gamma^{\mathrm{Lyc}, \mathrm{p} \rightarrow \mathrm{m}}$. A fraction of energy load of the ejecta $\left.e_{\mathrm{SN}} \frac{\partial \rho_{\mathrm{S}}}{\partial t}\right|_{\mathrm{FB}}$ is deposited into $\rho E$ (via $\rho e$ ) and $\rho K,\left(1-\epsilon_{\mathrm{SN}}\right)$ and $\epsilon_{\mathrm{SN}}$, respectively. However, this does not account for the kinetic energy of the ejecta that must also be transferred to the hydro-mesh along with their mass:

$\left.\frac{\partial\left(\rho_{\mathrm{s}} E_{\mathrm{kin}}\right)}{\partial t}\right|_{\mathrm{FB}}=\left.\sum_{p} \frac{\boldsymbol{u}_{\mathrm{s}, \mathrm{p}}^{2}}{2} \frac{\partial \rho_{\mathrm{s}}}{\partial t}\right|_{\mathrm{FB}, \mathrm{p}}$.

Here we sum over all local contributions to kinetic energy from every individual particles ' $p$ '. The momentum of the ejecta transferred to bulk momentum of the gas changes the bulk kinetic energy of the gas by

$\left.\frac{\partial\left(\rho E_{\mathrm{kin}}\right)}{\partial t}\right|_{\mathrm{FB}}=\left.\frac{\boldsymbol{u}}{2}\left(2 \boldsymbol{u}_{\mathrm{s}}-\boldsymbol{u}\right) \frac{\partial \rho_{\mathrm{s}}}{\partial t}\right|_{\mathrm{FB}}$.

MNRAS 442, 3407-3426 (2014) 
To conserve energy, we add the difference to $\rho K$

$$
\left.\frac{\partial(\rho K)}{\partial t}\right|_{\mathrm{FB}} ^{\mathrm{ex}}=\left.\frac{\partial\left(\rho E_{\mathrm{kin}}\right)}{\partial t}\right|_{\mathrm{FB}}-\left.\frac{\partial\left(\rho E_{\mathrm{kin}}\right)}{\partial t}\right|_{\mathrm{FB}} .
$$

The heating of the gas to very high temperatures by $\mathrm{SNe}$ is described by a hot phase density $\rho_{\mathrm{h}}$, obeying the conservation equation

$$
\begin{gathered}
\frac{\partial \rho_{\mathrm{h}}}{\partial t}+\nabla \cdot\left(\rho_{\mathrm{h}} \boldsymbol{u}\right)+\min \left[\rho_{\mathrm{h}} \nabla \cdot \boldsymbol{u}, 0\right] \\
=\left.\frac{\partial \rho_{\mathrm{s}}}{\partial t}\right|_{\mathrm{FB}}-\rho_{\mathrm{h}} \exp \left(-t / \tau_{\mathrm{h}}\right),
\end{gathered}
$$

where $\min \left[\rho_{\mathrm{h}} \nabla \cdot \boldsymbol{u}, 0\right]$ describes the loss of thermal energy due to adiabatic expansion, ${ }^{2}$ and $\rho_{\mathrm{h}} \exp \left(-t / \tau_{\mathrm{h}}\right)$ the decay of $\rho_{\mathrm{h}}$ due to successive mixing of the hot gas into the ISM. The half-lifetime scale $\tau_{\mathrm{h}} / \log (2)$ is defined such that a SNe bubble shell at typical expansion velocity (roughly the speed of sound in the hot phase) travels roughly $1 \mathrm{kpc}$ during that period, which leads to $\tau_{\mathrm{h}} \approx 1 \mathrm{Myr}$. $e_{\mathrm{h}} \approx 0.1 \times e_{\mathrm{SN}}$ is the constant specific thermal energy of the hot phase gas.

For consistency, the input parameters $\hat{\rho}_{\mathrm{w}}$ and $\hat{e}_{\mathrm{w}}$ to the derivation of the radiative cooling rate $\Lambda_{\mathrm{w}}^{\mathrm{rad}}$ (equation 16) are computed as follows:

$$
\begin{aligned}
& \hat{\rho}_{\mathrm{w}}=\rho_{\mathrm{w}}-\rho_{\mathrm{h}}, \\
& \hat{e}_{\mathrm{w}}=\frac{\rho_{\mathrm{w}} e_{\mathrm{w}}-\rho_{\mathrm{h}} e_{\mathrm{h}}}{\hat{\rho}_{\mathrm{w}}} .
\end{aligned}
$$

\section{SIMULATIONS}

\subsection{Initial conditions}

In the simulation domain with a volume of $0.5 \mathrm{Mpc}^{3}$ we initialize an isothermal gaseous disc with an exponential surface density profile residing in a static dark matter halo using the potential-method of Wang et al. (2010), which gives initial conditions similar to Agertz et al. (2009). The choice of this setup is advantageous compared to a setup using a constant vertical scale height of the disc alike that by Tasker \& Tan (2009), because it is adiabatically stable.

In the absence of a stellar component, the exponential gaseous disc is defined by its mass $M_{\text {gas }}=10^{10} \mathrm{M}_{\odot}$, its orientation of the disc angular momentum $\boldsymbol{n}_{\text {gas }}$ assuming a radial scale length $r_{\text {gas }}=$ $3.5 \mathrm{kpc}$, an initially uniform metallicity $Z_{\mathrm{gas}}=0.1 \times Z_{\odot}$ and a temperature $T_{\text {gas }}=4 \times 10^{4} \mathrm{~K}$ of the disc.

The dark matter is modelled by a static halo with a NFW-shaped density profile (Navarro, Frenk \& White 1997). It only contributes to the dynamics via its gravitational acceleration

$\boldsymbol{g}_{\mathrm{dm}}=\frac{-G M_{\mathrm{dm}} \boldsymbol{r}}{\log \left(1+c_{\mathrm{dm}}\right)-\frac{c_{\mathrm{dm}}}{1+c_{\mathrm{dm}}}}\left(\frac{\log \left(r_{\mathrm{s}}\right)}{r^{3}}-\frac{c_{\mathrm{dm}}}{r_{\mathrm{dm}} r^{2} r_{\mathrm{s}}}\right)$,

at a given position with distance vector $\boldsymbol{r}$ from the halo centre, its absolute value $r$ and the scaled dimensionless radius $r_{\mathrm{s}}=(1$ $\left.+r c_{\mathrm{dm}} / r_{\mathrm{dm}}\right)$. The NFW profile used is fully characterized by the halo mass $M_{\mathrm{dm}}=10^{12} \mathrm{M}_{\odot}$, the virial radius $r_{\mathrm{dm}}=213 \mathrm{kpc}$, and a concentration parameter $c_{\mathrm{dm}}=12$.

\subsection{Individual runs}

We performed eight isolated galaxy runs with model parameters listed in Table 2. All runs were carried out with a root grid of $256^{3}$

${ }^{2}$ Employing a ceiling $\left(\rho_{\mathrm{h}} \nabla \cdot \boldsymbol{u}\right)_{\max }=0$ prevents producing hot phase when gas is compressed.
Table 2. Simulation runs.

\begin{tabular}{lllllll}
\hline ID & \multicolumn{1}{ll}{ LES } & ISM & $\epsilon_{\mathrm{SN}}$ & $\rho_{\mathrm{h}}$ & Stop time \\
\hline ref & $30 \mathrm{pc}$ & Yes & MIST & 0.085 & Yes & $1.0 \mathrm{Gyr}$ \\
$\mathrm{nE}$ & $30 \mathrm{pc}$ & Yes & MIST & 0.0 & Yes & $1.0 \mathrm{Gyr}$ \\
$\mathrm{nB}$ & $30 \mathrm{pc}$ & Yes & MIST & 0.085 & No & $0.5 \mathrm{Gyr}$ \\
$\mathrm{nBnE}$ & $30 \mathrm{pc}$ & Yes & MIST & 0.0 & No & $0.4 \mathrm{Gyr}$ \\
$\mathrm{sSF}$ & $30 \mathrm{pc}$ & Yes & Simple & 0.0 & Yes & $0.8 \mathrm{Gyr}$ \\
$\mathrm{sSF} 2$ & $30 \mathrm{pc}$ & No & Simple & 0.0 & Yes & $0.4 \mathrm{Gyr}$ \\
lres5 & $60 \mathrm{pc}$ & Yes & MIST & 0.085 & Yes & $1.0 \mathrm{Gyr}$ \\
lres4 & $120 \mathrm{pc}$ & Yes & MIST & 0.085 & Yes & $1.0 \mathrm{Gyr}$ \\
\hline
\end{tabular}

cells. AMR levels with a factor of 2 spatial and temporal refinement were created using refinement criteria based on density; specifically, any cells with density above the minimum value of $0.01 \mathrm{M}_{\odot} \mathrm{pc}^{-3}$ were tagged for refinement up unto a specified maximum number of levels. In runs ref $\mathrm{nE}, \mathrm{nB}, \mathrm{nEnB}, \mathrm{sSF}$, and $\mathrm{sSF} 2$ a spatial resolution of $\sim 30 \mathrm{pc}$ was obtained using six levels of refinement. The effects of the feedback implementation are explored with the runs $\mathrm{nE}, \mathrm{nB}$, and $\mathrm{nEnB}$ in comparison with run ref, that features MIST with the reference parameters as given in Table 1 .

The runs SSF and SSF2 run feature a simplified model for the ISM without phase separation and $\epsilon_{\mathrm{SN}}=0$. A threshold controlled star formation recipe is applied here, according to which stars are allowed to form at a free-fall time efficiency of $\varepsilon_{\mathrm{sSF}}=0.01$, if the local density exceeds $\rho_{\mathrm{sSF}, \min }=50 \mathrm{~m}_{\mathrm{H}} \mathrm{cm}^{-3}$ and the local temperature is lower than $T_{\mathrm{sSF}, \max }=1.5 \times 10^{4} \mathrm{~K}$. While in sSF2 the whole SGS-turbulence model is switched off, the model is still active in sSF, but star formation is decoupled from $K$ and the SGS-energy production terms related to MIST are switched off in this case (i.e. $\Pi_{\mathrm{TI}} \equiv 0$ and $\Pi_{\mathrm{SN}} \equiv 0$ ). Stellar particles are not created as pairs (see Section 2.3.1) in $\mathrm{SSF}$ and $\mathrm{sSF} 2$, since $\ell_{\mathrm{c}}$ is not defined in both cases.

In addition two runs with fewer refinement levels were performed in order to investigate the effects of numerical resolution on the results of our simulations. The runs lres 5 and lres 4 feature effective resolutions of $\sim 60 \mathrm{pc}$ and $\sim 120 \mathrm{pc}$ using five and four levels of refinement, respectively.

Up to densities around $0.3 \mathrm{M}_{\odot} \mathrm{pc}^{-3}$ the resolution requirement by Truelove et al. (1997) is easily satisfied in all runs with MIST. This limit can be shifted towards much higher densities, if we consider the effective pressure instead of thermal pressure only. The Jeans length of the few densest cells may temporarily drop below $4 \Delta$ though, but never below the size of a cell $\Delta$, before the dense region is disrupted again by feedback. The latter statements are not true in case of SSF and SSF2, in which the Jeans length criterion is always violated in the dense clusters.

The combined usage of LES and MIST in a simulation increases the amount of computational resources required by less than 10 per cent compared to runs without, and significantly less than an additional level of refinement (more than 100 per cent).

The isolated galaxies were evolved for at least one orbital time at $10 \mathrm{kpc}$ radius from the centre ( $\sim 400 \mathrm{Myr})$.

\section{RESULTS}

\subsection{Disc evolution in the ref run}

Initially the disc is adiabatically stable, but as the gas is allowed to cool by radiation, it loses its thermal support in height and collapses into a thin cold disc. The disc becomes Toomre-unstable and fragments into clumps. In those clumps the gas eventually becomes dense enough to become molecular and consequently begins to form 
3414 H. Braun et al.
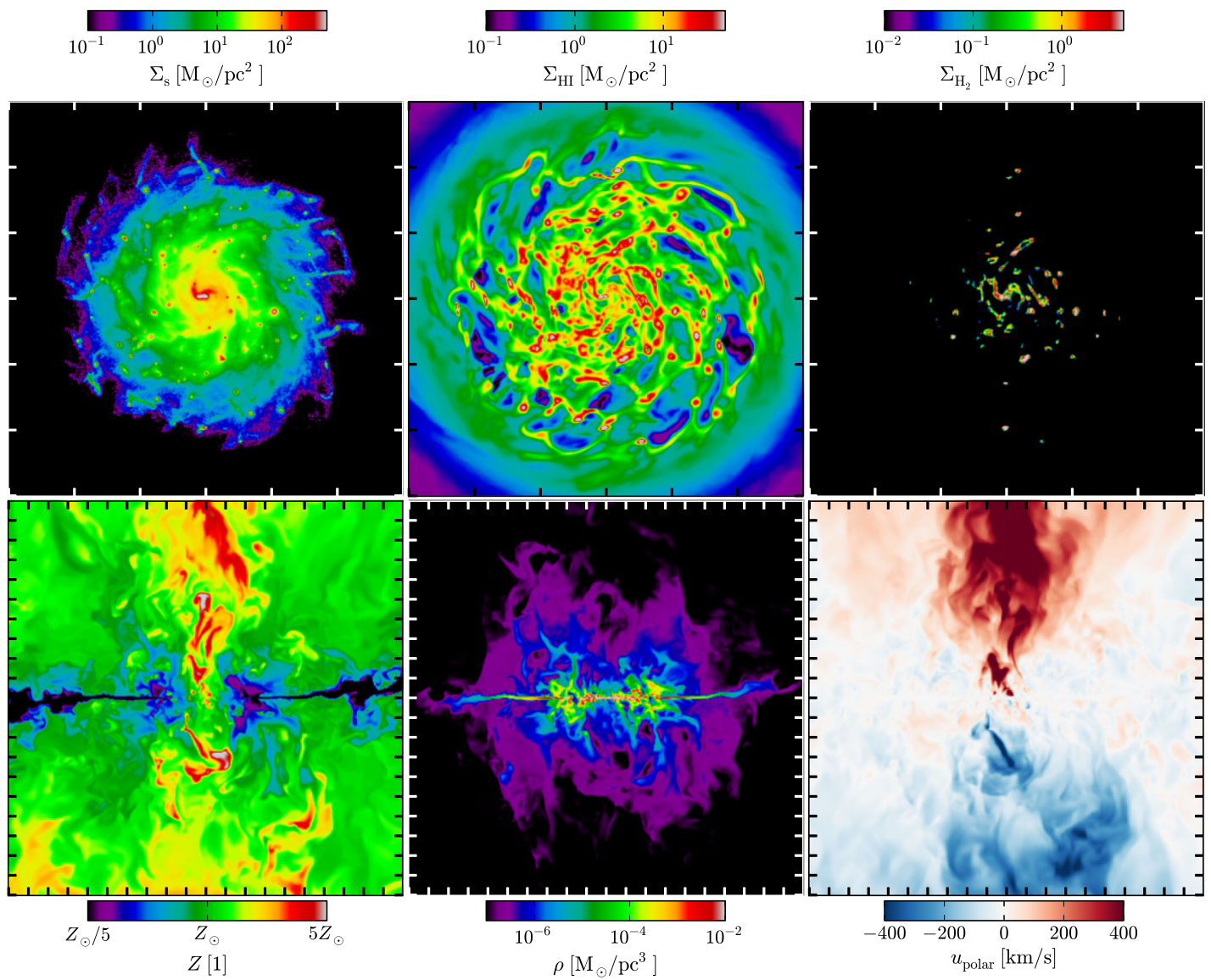

Figure 1. The top row of panels shows the following projected quantities in the central $(30 \mathrm{kpc} \times 30 \mathrm{kpc})$ region of the galactic disc of the ref run after $1 \mathrm{Gyr}$ (left to right): stellar column density $\Sigma_{\mathrm{s}}, \mathrm{H}_{\mathrm{I}}$ column density $\Sigma_{\mathrm{H}}$, star formation column density $\dot{\Sigma}_{\mathrm{SF}}$. The bottom row shows polar $(100 \mathrm{kpc} \times 100 \mathrm{kpc})$ slices of the central region of the galactic disc of the ref run at $1 \mathrm{Gyr}$ (left to right): metallicity $Z$, total gas density $\rho$, velocity perpendicular to the disc plane $v_{\text {polar }}$. The tick marks on the plot edges have a spacing of $5 \mathrm{kpc}$.

stars. The SNe feedback of the newly formed stars then eventually disperses the clumps. A fraction of the stars formed in some of those clumps may form a stellar cluster that survives over a much longer time than the lifetime of about $20 \mathrm{Myr}$ of the gas clump from which it originated. The stellar clusters tend to move towards the centre of the disc as a result of dynamical friction, where they eventually merge into a central agglomeration of stars, if they are not disrupted before by the tidal forces in the disc. However, they do not build up a bulge as their velocity dispersion is too small. The majority of the stars form a rather smooth disc with a scale height of a few hundred parsec. The structure of the stellar disc is shown in the top left panel of Fig. 1. The stars stripped in the potential of the disc form tidal tails around their birth cluster.

The SNe also carve holes into the disc and launch a wind leaving the disc. The interplay between cooling, gravity, and SNe shapes the gaseous component into a fluffy disc, with holes and knots, as demonstrated in the top and bottom panels in the middle of Fig. 1. The wind mostly not only consists of metal-enriched hot gas from $\mathrm{SNe}$, but also carries a fraction of the original cold clump with it (see bottom left panel of Fig. 1). The wind originates from the cavities of the disc caused by the $\mathrm{SNe}$ at speeds ranging from 300 to $1000 \mathrm{~km} \mathrm{~s}^{-1}$, and pushes a shell of cold or warm gas outward. The ejected gas is either mixed into the wind, or falls back into the disc. Far away from its origin, the wind from all sources merges into a hot, but dilute, sub-sonically turbulent, and metal-rich $\left(\sim \mathrm{Z}_{\odot}\right)$ bubble that continues to expand. The bottom panels of Fig. 1 give an impression of the metallicity, mass, and velocity structures in the winds above the disc.

As seen in the top right panel of Fig. 1, active star formation occurs only in a few compact regions away from the centre. Because of the local metal enrichment due to $\mathrm{SNe}$ ejecta, the threshold density for star formation drops. This causes clumps to form stars earlier during their collapse, when the density is still relatively low, which lowers their star formation rate and makes them more prone to dispersal by SNe. The residual stellar clusters are fewer, lighter, and more easily disrupted in the galaxy's potential.

The global star formation rate in the ref run is plotted as black line in Fig. 2. Initially the amount of star formation increases quickly, as the region of star formation grows. It reaches its peak around $300 \mathrm{Myr}$ after start of simulation, and then gradually declines due 


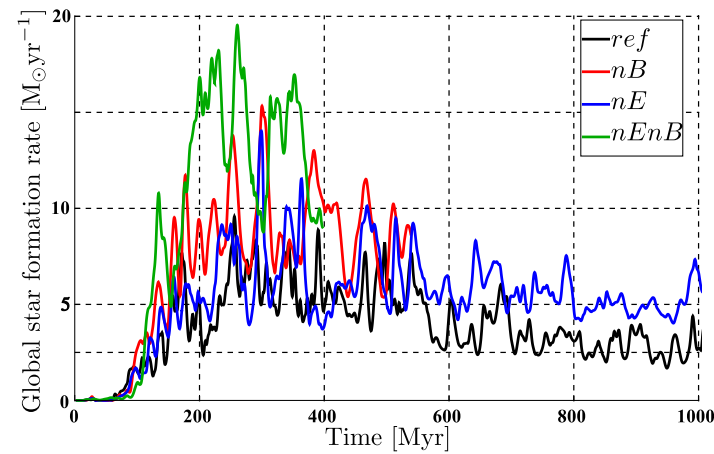

Figure 2. Global star formation rate $\dot{M}_{\mathrm{SF}}$ over simulation time for the different runs ref, $\mathrm{nB}, \mathrm{nE}$, and $\mathrm{nEnB}$ in black, red, blue, and green, respectively.

the consumption and the metal enrichment of the gas reservoir in the inner disc. At this stage approximately 30 per cent of the initial gas mass has been converted into stars.

\subsubsection{Differences in the $n E$ run}

In the $\mathrm{nE}$ run we set $\epsilon_{\mathrm{SN}}=0$. Thus $\mathrm{SNe}$ feedback does not directly increase the unresolved turbulent energy $K$ (see equation 5). This has basically two effects on the overall evolution. On the one hand, star formation in a clump is active over a longer period of time because of the higher star formation efficiency in moderately turbulent clumps $\left(\mathcal{M}_{\mathrm{c}} \sim 10\right)$, causing more stars to form before a clump of gas is dispersed. This leads to a slightly higher global star formation rate than in the ref run (see the blue line in Fig. 2). On the other hand, stars forming in a clump after the first stars have already produced $\mathrm{SNe}$ have a significantly reduced velocity dispersion compared to their analogues from the ref run. As a consequence, those stars are more likely to stay near the clump of their origin. In this case, stellar clusters tend to be more massive and more strongly gravitationally bound, and hence, their tidal tails are less prominent. Clusters are initially more abundant and have a longer lifetime. Eventually they merge into a few very massive clusters, which accrete gas, and host intermittent star formation, as the gas is driven apart due to feedback. They remain gravitationally bound and stable, as their stellar mass is sufficiently large and concentrated.

As a consequence, the resulting stellar and gaseous discs are more clumpy than in the ref run. This can be seen by comparing the projections of total gaseous density and stellar density in Fig. 3, where both stellar and gaseous clumps are fewer in numbers of appearance but more massive with higher central densities. During the simulation approximately 40 per cent of the initial gas mass was consumed by star formation after $1 \mathrm{Gyr}$.

\subsubsection{Differences in the $n B$ run}

In the $\mathrm{nB}$ run we turned off the treatment of the hot $\mathrm{SN}$ ejecta phase, i.e. the hot $\mathrm{SN}$ gas is directly mixed into the warm phase. This allows the gas to radiate away the feedback energy more quickly. Thus star formation in a clump goes on for a longer time, consuming a larger fraction of the gaseous mass. Once almost all gas is depleted the feedback takes the lead, and drives stronger and faster winds than in the ref run. Like in the $\mathrm{nE}$ run, the resulting stellar clusters are more stable and massive, and subsequently merge to form very massive clusters, that cannot be disrupted in the tidal field of the disc. Those

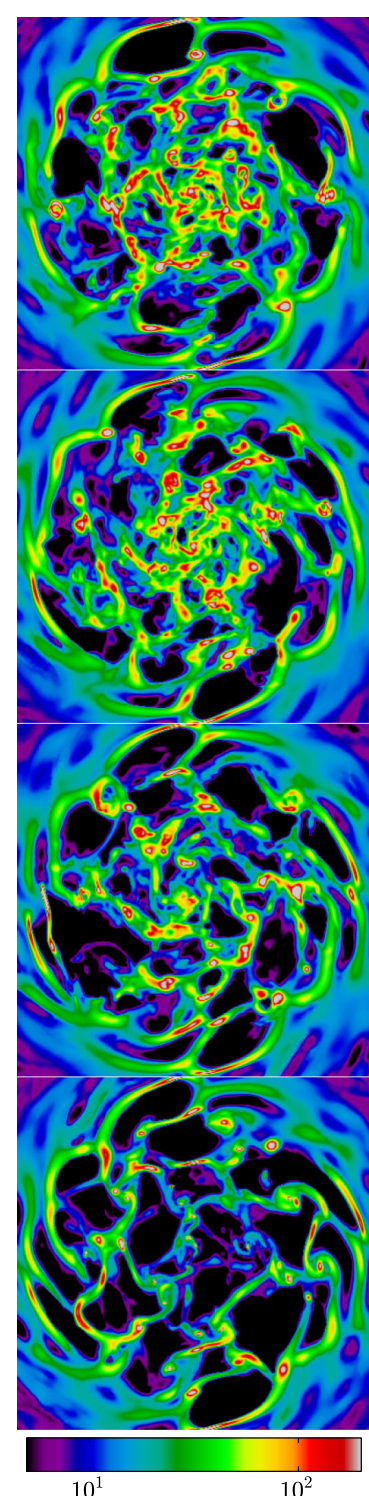

$10^{1}$
total gas $\Sigma\left[\mathrm{M}_{\odot} / \mathrm{pc}^{2}\right]$
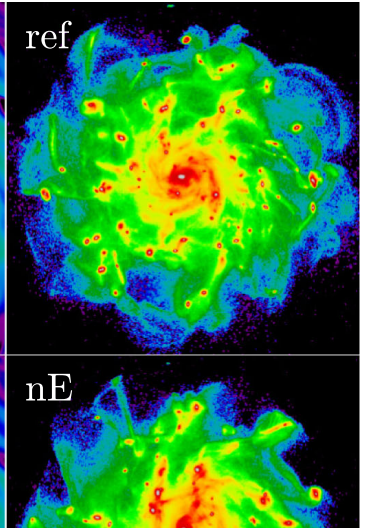

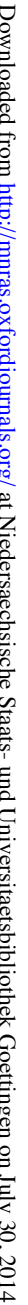

Figure 3. Comparison of total gas surface density $\Sigma$ in the left column and the stellar surface density $\Sigma_{\mathrm{s}}$ in the right column between the different runs (from top to bottom: ref, nE, nB, nEnB) $400 \mathrm{Myr}$ after start of simulation.

massive clusters are hosting continuous star formation. They keep accreting gas from the disc and converting it into stars, thereby sustaining their gaseous mass at the same level for a long time.

As in the $\mathrm{nE}$ run, the resulting gaseous and stellar discs are clumpy, but the stellar clusters are much more massive, and the gas is depleted on much shorter time-scales (see Fig. 3). The stellar density in the centre of the clusters reaches values much greater than $10^{3} \mathrm{M}_{\odot} \mathrm{pc}^{-3}$. This is why we stopped this simulation after 


\section{H. Braun et al.}

0.5 Gyr. During this period of time about 30 per cent of gaseous mass was turned into stars.

\subsubsection{Differences in the $n E n B$ run}

Setting $\epsilon_{\mathrm{SN}}=0$ and turning off the treatment of the hot SN ejecta phase combines the effects described above. This leads to an even more violent evolution with a few very massive stellar clusters in the inner regions, while the gas is depleted quickly (see Fig. 3). We stopped this run even earlier than the $\mathrm{nB}$ run. The amount of gas consumed by star formation was about 30 per cent in roughly 400 Myr.

\subsection{Star formation}

\subsubsection{Time scales}

The $\sim 20$ Myr time-scale related to single star-forming regions reflects the period of time needed to produce enough stars, such that their combined feedback is able to quench star formation, and to evaporate the most dense, central region of the star-forming cloud. If the environment is still dense enough, star formation may continue in an compressed layer around the expanding bubble. Depending on the mass of the clump and its surroundings, star formation can continue in this mode. A time series depicting the evolution of one of those clumps is shown in Fig. 4. The impact of the most massive clumps is seen in the quick variations of the global star formation rate $\dot{M}_{\mathrm{SF}}$ on time-scales around 10 to $40 \mathrm{Myr}$ (see Fig.

5). Metal-enriched clouds tend to have a shorter lifetime compared to metal-poor clouds, as star formation can start at lower densities, because shielding from radiation is enhanced due to higher dust abundances.

By assuming different threshold densities $\rho_{\mathrm{thr}, \mathrm{i}}=\{0.0,0.01,0.1$, $0.32,1.0\} \mathrm{M}_{\odot} \mathrm{pc}^{-3}$ to compute the reservoir of available gas, we derive global gas depletion time-scales

$\tau_{\mathrm{dep}, \mathrm{i}}(t)=\frac{\sum_{\rho(t)>\rho_{\mathrm{thr}, \mathrm{i}}} \rho \Delta^{3}}{\sum \dot{\rho}_{\mathrm{SF}} \Delta^{3}}=\frac{M_{\mathrm{gas}, \mathrm{i}}}{\dot{M}_{\mathrm{SF}}}$.

To filter out quick variations, we apply a moving average

$\bar{\tau}_{\mathrm{dep}, \mathrm{i}}(t)=\frac{1}{t_{\mathrm{av}}} \int_{t-t_{\mathrm{av}} / 2}^{t+t_{\mathrm{av}} / 2} \tau_{\mathrm{dep}, \mathrm{i}}(\grave{t}) \mathrm{d} \grave{t}$

with $t_{\mathrm{av}}=50 \mathrm{Myr}$. Plots of the resulting depletion times $\tau_{\mathrm{dep}, \mathrm{i}}$ are shown in Fig. 5 for different choices of $\rho_{\text {thr, i }}$. The typical time scales range from less than $100 \mathrm{Myr}$ for the highest threshold of $1 \mathrm{M}_{\odot} \mathrm{pc}^{-3}$ to a few Gyr for the total gas content of the simulation domain. Gas above densities $\rho_{\text {thr }}=1 \mathrm{M}_{\odot} \mathrm{pc}^{-3}$ is most likely actively star forming. Contrary to the other cases, $\tau_{\text {dep }}$ for $\rho_{\text {thr }}=$ $1 \mathrm{M}_{\odot} \mathrm{pc}^{-3}$ slightly shrinks with time. Because of the increasing metallicity the threshold density needed for star formation drops gradually. Star formation and subsequent feedback then prevent gas from becoming as dense as in metal-poorer environments. $\tau_{\text {dep }}$ for $\rho_{\text {thr }}=0.32 \mathrm{M}_{\odot} \mathrm{pc}^{-3}$ remains almost constant at $0.25 \mathrm{Gyr}$ after the initial transient phase. For lower $\rho_{\text {thr }}, \tau_{\text {dep }}$ grows with time, and the growth rate tends to increase with decreasing $\rho_{\mathrm{thr}}$. The growth of $\tau_{\text {dep }}$ if $\rho_{\text {thr }} \leq 0.32 \mathrm{M}_{\odot} \mathrm{pc}^{-3}$ is also related to the increase of metallicity. In metal-rich environments gas forms stars already at lower densities, implying greater $\tau_{\mathrm{ff}, \mathrm{c}}$, but the efficiency $\epsilon_{\mathrm{PN}}$ (see equation (17)) remains about the same. For this reason $\dot{M}_{\mathrm{SE}}$ is effectively lowered faster than gas supply $M_{\text {gas }}$ shrinks. We expect that all $\tau_{\text {dep }}$ will saturate, as the impact of increasing metallicity is further reduced in already enriched gas (see BS12). However, the magnitude

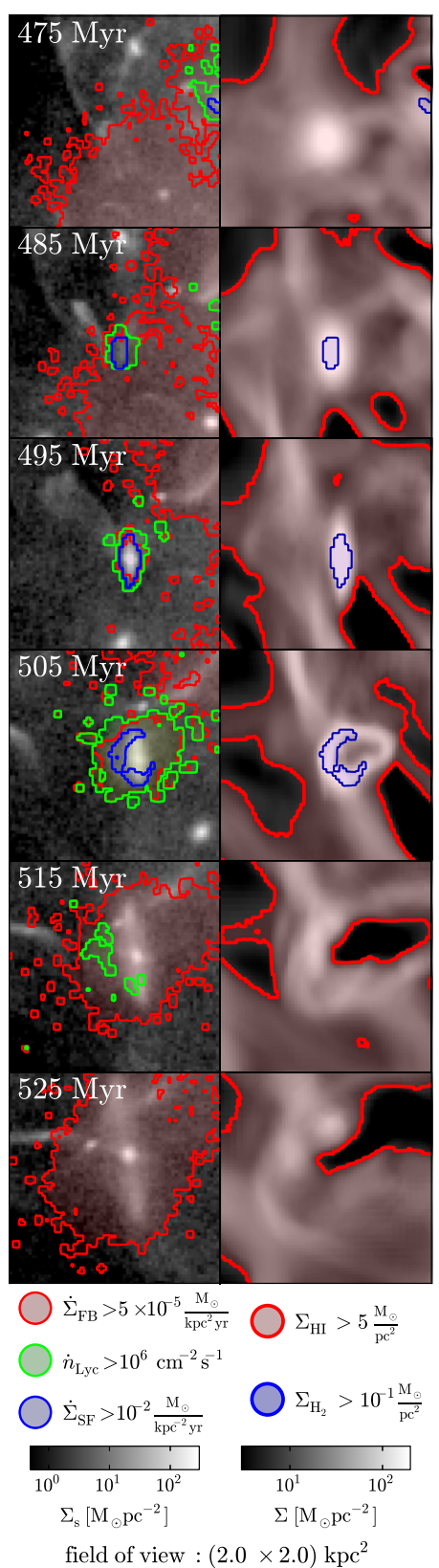

Figure 4. Evolution of a gas clump from shortly before star formation begins until dispersal. In the left panels the stellar surface density $\Sigma_{\mathrm{s}}$ in grey-scales is over-plotted with contours of the surface density of the $\mathrm{SNe}$ feedback $\dot{\Sigma}_{\mathrm{FB}}$ in red (indicating stellar populations at ages between $\sim 4$ and $\sim 40 \mathrm{Myr}$ ), the surface density of Lyman continuum emission $\dot{n}_{\text {Lyc }}$ in green (indicating stellar populations younger than $\sim 2 \mathrm{Myr}$ ), and the star formation surface density $\Sigma_{\mathrm{SF}}$ in blue. Right panels show the total gas surface density $\Sigma$ in grey-scales over-plotted with contours of surface density of cold gas $\Sigma_{\mathrm{HI}_{\mathrm{I}}}$ in red, and the surface density of dense molecular gas $\Sigma_{\mathrm{H}_{2}}$ in blue. 


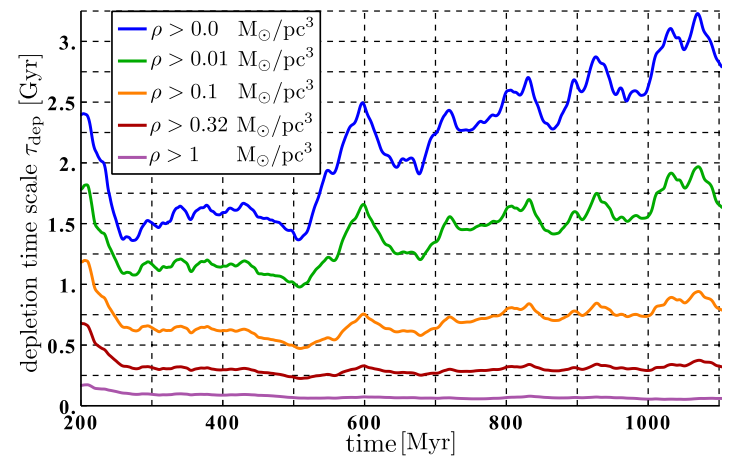

Figure 5. Global gas depletion time-scale $\tau_{\text {dep }}$ versus time in the ref run. We plot moving averages of $\tau_{\text {dep }}$ to eliminate short period variations caused by single star-forming regions. $\tau_{\mathrm{dep}}$ was derived with respect to gaseous mass in regions with $\rho>\rho_{\text {thr }}$. Blue, green, orange, red, and purple lines show $\tau_{\text {dep }}$ using threshold densities $\rho_{\text {thr }}=\{0.0,0.01,0.1,0.32,1\} \mathrm{M}_{\odot} \mathrm{pc}^{-3}$, or in terms of number density $n_{\mathrm{thr}} \simeq\{0.0,0.33,3.3,10,33\} \mathrm{cm}^{-3}$.

of the depletion time-scales is well within range of observationally inferred ones (Daddi et al. 2010; Genzel et al. 2010).

\subsubsection{Local efficiency}

The star formation efficiency is defined by

$$
\dot{\rho}_{\mathrm{SF}}=\frac{\epsilon_{\mathrm{ff}} \rho}{\tau_{\mathrm{ff}}}
$$

where a constant value for $\epsilon_{\mathrm{ff}} \simeq 0.01$ is commonly assumed. In our simulations $\epsilon_{\mathrm{ff}}$ is computed dynamically from the local turbulent hydrodynamical state. While the star formation efficiency in the cold phase, $\epsilon_{\mathrm{PN}}$ (see equation 17), is almost constant - around $\epsilon_{\mathrm{PN}}$ $\simeq 0.1$ - for all star-forming regions, the shielded molecular content $f_{\mathrm{H}_{2}} \rho_{\mathrm{c}}$ and $\rho_{\mathrm{c}, \mathrm{pa}}$ vary significantly, such that $\epsilon_{\mathrm{ff}}$ is boosted in highdensity regions. As a consequence, a large contribution to the global star formation rate comes from just a few temporarily very active spots in the disc, which explains the relatively large variations on short time-scales. As shown in Fig. 6, we find a good correlation $\epsilon_{\mathrm{ff}} \propto \sqrt{\dot{\rho}_{\mathrm{SF}}}$, which holds for all times in the ref run and the $\mathrm{nE}$ run. In the other runs without treatment of a hot phase, the power-law slope flattens towards large $\dot{\rho}_{\mathrm{SF}}$, which is clearly a sign that the effectiveness of thermal feedback plays a major role. The typical value of $\epsilon_{\mathrm{ff}} \simeq 0.01$ is, on average, reproduced in runs with hot phase treatment, while those without produce stars at significantly higher average efficiency.

\subsection{3 $\mathrm{H}_{2}$ and $\mathrm{H}_{\mathrm{I}}$}

We find a very tight correlation between the star formation column density $\dot{\Sigma}_{\mathrm{SF}}$ and the molecular column density $\Sigma_{\mathrm{H}_{2}}$, as shown on the left panel of Fig. 7. This correlation implies a robust power-law relation between $\dot{\Sigma}_{\mathrm{SF}}$ and $\Sigma_{\mathrm{H}_{2}}$ with an exponent $\alpha_{\mathrm{H}_{2}, \mathrm{SF}}=1.05 \pm 0.06$ slightly above one. This corresponds to an almost constant depletion time of the molecular gas in star-forming regions around $80 \mathrm{Myr}$. This correlation holds for all runs independent of the simulation time, as shown in the middle and right panels of Fig. 7.

The existence of the correlation itself is not surprising, as it is assumed in the MIST model equations that $\dot{\rho}_{\mathrm{SF}}$ is proportional to $f_{\mathrm{H}_{2}}$ (see equation 17). $\alpha_{\mathrm{H}_{2}, \mathrm{SF}} \simeq 1$, however, is not imposed. Assuming a constant star formation efficiency $\epsilon_{\mathrm{PN}}$, one would expect $\alpha_{\mathrm{H}_{2}, \mathrm{SF}} \simeq 1.5$, but in our model $\epsilon_{\mathrm{PN}}$ and $f_{\mathrm{H}_{2}}$ depend implicitly and non-linearly on $\rho_{\mathrm{c}, \mathrm{pa}}, \mathcal{M}_{\mathrm{c}}$, and $l_{\mathrm{c}}$, which are in turn non-linear functions of $\rho, \rho_{\mathrm{c}}, \rho_{\mathrm{w}}, K$, and $e_{\mathrm{w}}$. BS12 already showed that a correlation with $\alpha_{\mathrm{H}_{2}, \mathrm{SF}} \simeq 1$ can be found in the equilibrium solutions of the MIST model equations. This suggests that the interplay of the implicit dependencies effectively results in an almost linear relation between estimated $\mathrm{H}_{2}$-mass available to star formation and the star formation rate, which is an important feature of the self-regulating mechanisms implemented in MIST. The depletion time-scale of molecular gas from the equilibrium solutions (BS12) is about 1-2 Gyr, in agreement with that found by e.g. Bigiel et al. (2011) from CO-observations. We derive an average depletion time of $\sim 80 \mathrm{Myr}$ in our simulations, or shorter $(\sim 40 \mathrm{Myr})$ in regions of higher molecular density. This is considerably, by a factor of around

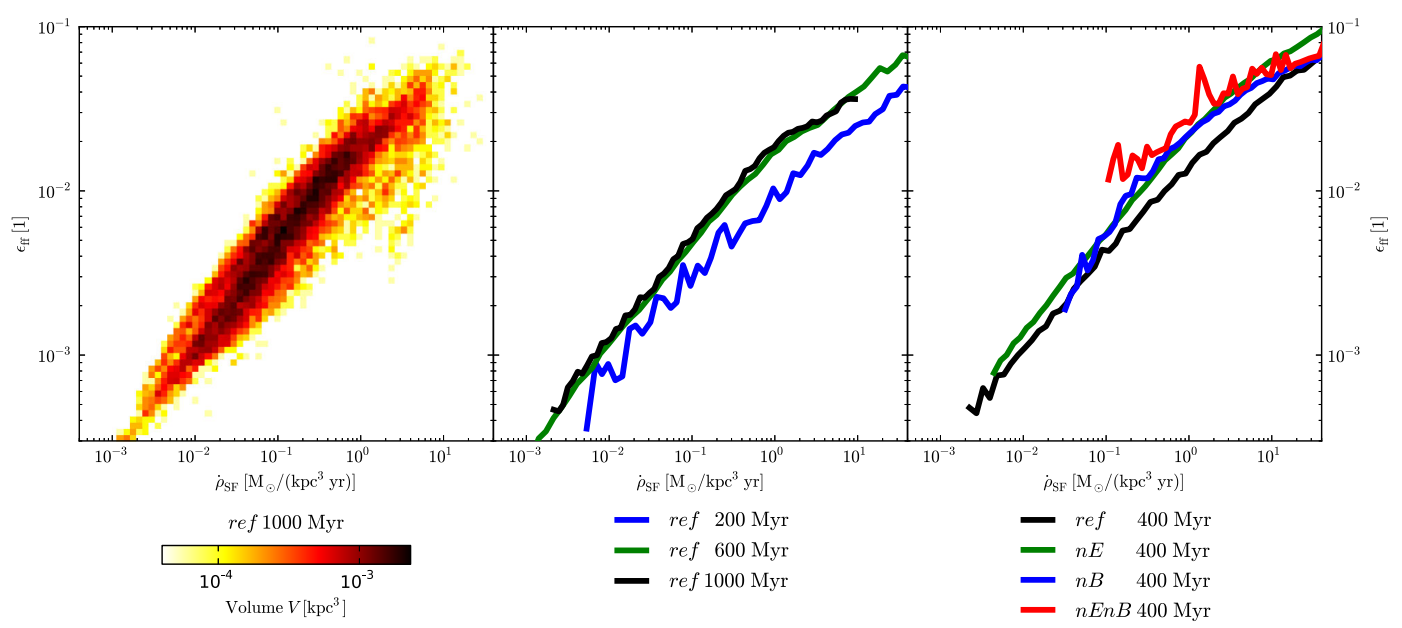

Figure 6. Star formation efficiency $\epsilon_{\mathrm{ff}}$ over star formation density $\dot{\rho}_{\mathrm{SF}}$. The left panel shows a two-dimensional area weighted histogram of the ref-data after 1 Gyr. The middle panel shows a time series (blue: $200 \mathrm{Myr}$, green: $600 \mathrm{Myr}$, black: $1 \mathrm{Gyr}$ ) of the mean $\epsilon_{\mathrm{ff}}$ corresponding to a specific value of $\dot{\rho}_{\mathrm{SF}}$, while on the right panel a comparison of the latter between the individual runs (black: ref, green: $\mathrm{nE}$, blue: $\mathrm{nB}$, red: $\mathrm{nEnB}$ ) at $400 \mathrm{Myr}$ is plotted. 


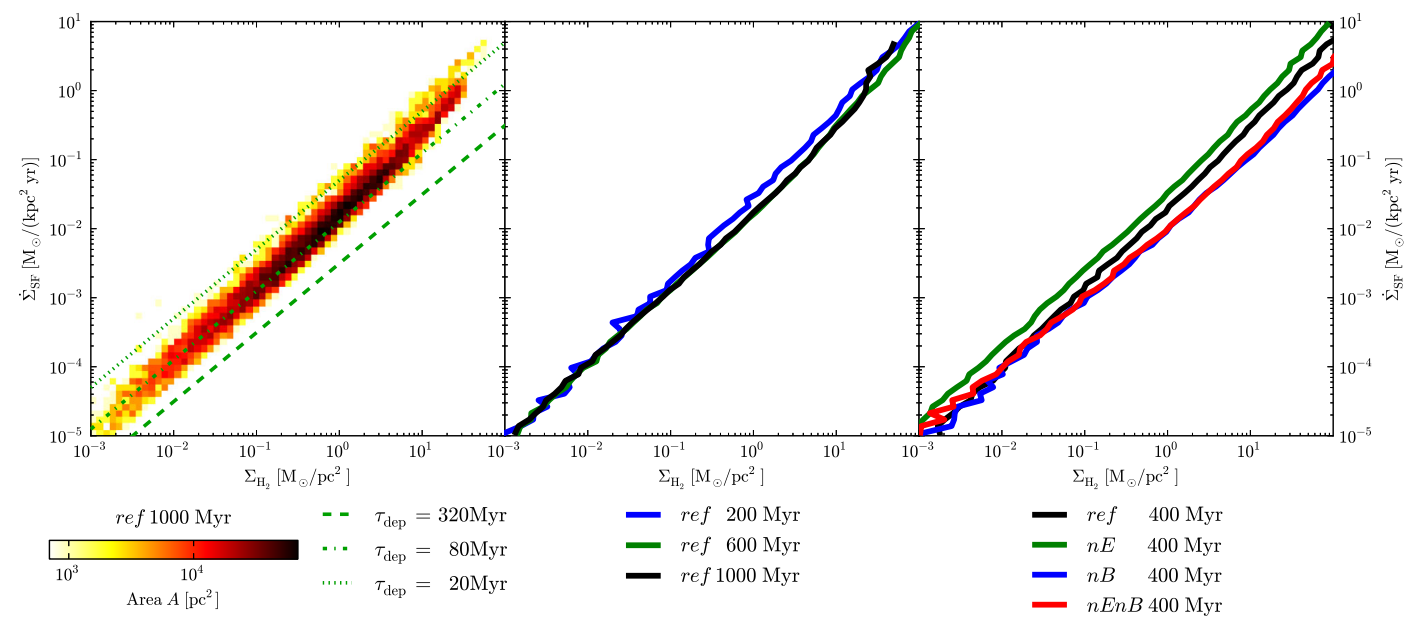

Figure 7. Star formation column density $\dot{\Sigma}_{\mathrm{SF}}$ over $\mathrm{H}_{2}$ column density $\Sigma_{\mathrm{H}_{2}}$. Plots and colours are arranged as in Fig. 6 . Additionally, in the left panel the dotted, dash-dotted, and dashed green lines indicate constant depletion time-scales of 20, 80, and $320 \mathrm{Myr}$, respectively.

25, shorter (see Fig. 7). But this time-scale matches the depletion time of molecular gas within star-forming molecular clouds, found by Murray (2011), ${ }^{3}$ and that for dense molecular gas inferred from HCN-observations by Gao \& Solomon (2004) ${ }^{4}$ and the total gas depletion time-scale $\tau_{\text {dep }}$ for $\rho_{\text {thr }}=1 \mathrm{M}_{\odot} \mathrm{pc}^{-3}$. This discrepancy between the depletion time-scales of molecular gas is caused by the fact that we estimate the amount of gas involved in the processes of active star formation by calculating the molecular fraction of gas in the centres of cold cloud complexes in equilibrium with an external radiation field. However, there are also environments containing significant amounts of $\mathrm{H}_{2}$ that are not in equilibrium with radiation or only partially shielded. Molecular material that is transported from shielded to not shielded regions by turbulent motions or $\mathrm{SNe}$ blast waves without being instantaneously dissociated is not tracked in our model for example. This material, as well as gas that is partially molecular but not dense enough to form stars, contributes to observed molecular hydrogen column densities, but is not present in our simulations. Our data on the $\mathrm{H}_{2}$-content of the ISM do reflect the amount of dense molecular gas that is actively forming stars, but do not reproduce the full amount of molecular gas seen in $\mathrm{CO}$-observations. Coarse graining our $\mathrm{H}_{2}$-data changes neither the inferred depletion time-scales nor the slope $\alpha_{\mathrm{H}_{2}, \mathrm{SF}}$ significantly, which is consistent with Gao \& Solomon (2004), who used galactic quantities.

Without following the chemical evolution of molecular and atomic gas, we cannot, unfortunately, distinguish between atomic gas and gas that would observationally be considered molecular. So we focus on the combined $\mathrm{H}_{\mathrm{I}}+\mathrm{H}_{2}$ content. The general shape of $\dot{\Sigma}_{\mathrm{SF}}$ over $\mathrm{H}_{\mathrm{I}}+\mathrm{H}_{2}$ shown in the left panel of Fig. 8 is simi-

${ }^{3}$ Murray (2011) find a GMC star-forming efficiency $\epsilon_{\mathrm{GMC}} \simeq 0.12$ and related free-fall times $\tau_{\mathrm{ff}, \mathrm{GMC}} \simeq 10 \mathrm{Myr}$. A combination of both yields a depletion time-scale of $\tau_{\text {dep, GMC }} \simeq 80 \mathrm{Myr}$.

${ }^{4} \mathrm{Gao} \&$ Solomon (2004) find a linear correlation between the galactic star formation rate $\dot{\rho}_{\mathrm{SF}, \text { gal }}$ and the amount $M_{\text {dense }}$ of what they call 'dense gas' in a galaxy: $\dot{\rho}_{\mathrm{SF}, \text { gal }}=1.8 M_{\mathrm{dense}} / 10^{8} \mathrm{yr}^{-1}$. This corresponds to a dense gas depletion time of $\sim 60 \mathrm{Myr}$. lar to observational relations (e.g. in Schruba et al. 2010), but our distribution is shifted towards higher densities and rates.

As demonstrated by Schruba et al. (2010), the distribution in $\dot{\Sigma}_{\mathrm{SF}}-\Sigma_{\mathrm{H}_{\mathrm{I}+\mathrm{H}_{2}}}$ space changes from low to high $\Sigma_{\mathrm{H}_{\mathrm{I}}+\mathrm{H}_{2}}$ between the vertical 'barrier' behaviour in $\dot{\Sigma}_{\mathrm{SF}}-\Sigma_{\mathrm{HI}}$ space and the linear relation observed for $\mathrm{H}_{2}$. This transition happens around the critical density for atomic to molecular conversion, which is metallicity dependent. Increasing the density of gas only leads to an increase in the molecular density, if its density was already above the critical density. Because of that the atomic density saturates. This explains the 'vertical barrier'-shaped distribution in $\dot{\Sigma}_{\mathrm{SF}}-\Sigma_{\mathrm{HI}}$ space, if we take into account that the amount of surrounding atomic gas does not affect star formation in the molecular gas.

From the high-density tail in the left panel of Fig. 8 one would rather infer a power-law slope $\alpha_{\mathrm{SF}, \mathrm{H} \mathrm{I}+\mathrm{H}_{2}} \simeq 1.5$ than a linear relation with $\alpha_{\mathrm{SF}, \mathrm{HI}+\mathrm{H}_{2}} \simeq 1$, but also a power-law slope $\alpha_{\mathrm{SF}, \mathrm{H}+\mathrm{H}_{2}} \simeq 2$ seems possible. At this point we cannot distinguish whether the reason for this is either that stellar feedback prevents the high-density tail from being populated up to densities at which $\alpha_{\mathrm{SF}, \mathrm{H} I+\mathrm{H}_{2}} \simeq 1$ could be observed, or the super-linear relation also seen in the equilibrium solutions of BS12 is recovered. With increasing metallicity of the star-forming gas the critical density of the atomic to molecular transition drops gradually. This effect is demonstrated by the shift towards lower $\Sigma_{\mathrm{H}_{\mathrm{I}+\mathrm{H}_{2}}}$ with increasing time in the middle panel of Fig. 8. A linear fit to the tail of the $\dot{\Sigma}_{\mathrm{SF}}-\Sigma_{\mathrm{HI}_{\mathrm{I}} \mathrm{H}_{2}}$ distribution in ref after $1 \mathrm{Gyr}$ yields a gas depletion time $\tau_{\mathrm{dep}, \mathrm{H}+\mathrm{H}_{2}} \simeq 400 \mathrm{Myr}$, roughly consistent with $\tau_{\text {dep }}\left(0.32 \mathrm{M}_{\odot} \mathrm{pc}^{-3}>\rho_{\text {thr }}>0.1 \mathrm{M}_{\odot} \mathrm{pc}^{-3}\right)$ (see Section 4.2.1). In the ref run roughly the same amount of stars and metals were produced after $1 \mathrm{Gyr}$ as in the $\mathrm{nEnB}$ after $0.4 \mathrm{Gyr}$. Nevertheless the $\dot{\Sigma}_{\mathrm{SF}}-\Sigma_{\mathrm{H} \text { I }+\mathrm{H}_{2}}$ relations are very different. While the high-density tail indicating the transition from $\mathrm{H}_{\mathrm{I}}$ to $\mathrm{H}_{2}$ becomes shallower and more prominent in the course of the disc evolution, the shape of $\dot{\Sigma}_{\mathrm{SF}}$ over $\Sigma_{\mathrm{H}_{+} \mathrm{H}_{2}}$ in the $\mathrm{nEnB}$ run at $0.4 \mathrm{Gyr}$ is about the same as in the early stages of ref. This is a consequence of inefficient mixing of the hot, metal-rich material from $\mathrm{SNe}$ with cold dense, but still metal-poor material that possibly could be turned into stars. The shift between the $\dot{\Sigma}_{\mathrm{SF}}-\Sigma_{\mathrm{H}_{\mathrm{I}} \mathrm{H}_{2}}$ relations for different runs in the right panel of Fig. 8 is mainly caused by gas consumption, as the 


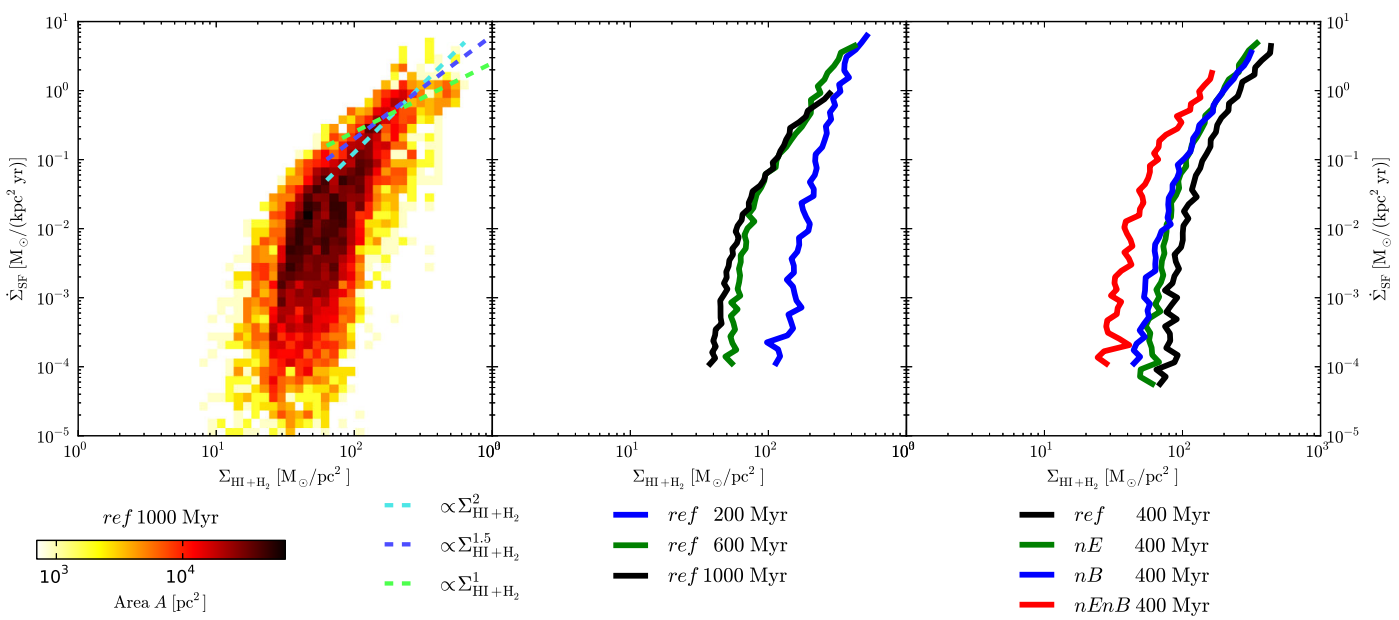

Figure 8. Star formation column density $\dot{\Sigma}_{\mathrm{SF}}$ over $\mathrm{H}_{\mathrm{I}}+\mathrm{H}_{2}$ column density $\Sigma_{\mathrm{H}_{\mathrm{I}}+\mathrm{H}_{2}}$. Plots and colours are arranged as in Fig. 6. Fits $\dot{\Sigma}_{\mathrm{SF}} \propto \Sigma_{\mathrm{H} I+\mathrm{H}_{2}}^{2}$, $\dot{\Sigma}_{\mathrm{SF}} \propto \Sigma_{\mathrm{HI}+\mathrm{H}_{2}}^{1.5}$, and $\dot{\Sigma}_{\mathrm{SF}} \propto \Sigma_{\mathrm{HI}+\mathrm{H}_{2}}^{1}$ are shown as dashed cyan, dashed blue, and dashed green line, respectively.

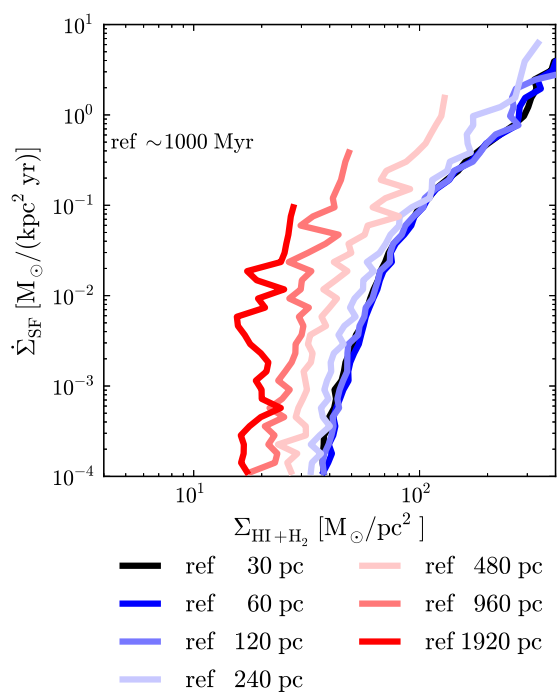

Figure 9. Star formation column density $\dot{\Sigma}_{\mathrm{SF}}$ over $\mathrm{H}_{\mathrm{I}}+\mathrm{H}_{2}$ column density $\Sigma_{\mathrm{HI}+\mathrm{H}_{2}}$ for different resolution scales $\Delta_{\text {crse }}=$ $\{30,60,120,240,480,960,1920\} \mathrm{pc}$ as solid lines. The full resolution data from the ref run were coarsened by the appropriate powers of two. Additionally, data from 10 consecutive root grid time steps (separated by $\mathrm{d} t \simeq$ $0.65 \mathrm{Myr}$ ) around a simulation time of $1 \mathrm{Gyr}$ were averaged to reduce scatter.

produced metals have not been mixed into the star-forming material yet. So, the shift between our results and observational findings is, on the one hand, a consequence of the lower metallicity and higher gas contents, compared to observed local galaxies.

On the other hand the resolution scale $\Delta_{\text {crse }}$, at which the relation between star formation and gas density is evaluated, influences the critical density of the atomic to molecular transition too, as demonstrated in Fig. 9. Once the averaging scale is considerably larger than a typical star-forming region, i.e. above $120 \mathrm{pc}$ in the ref run, the transition range in $\dot{\Sigma}_{\mathrm{SF}}-\Sigma_{\mathrm{HI}+\mathrm{H}_{2}}$ space seems to be shifted towards lower densities. Observational data like in Bigiel et al. (2011) usually correspond to averages over much larger areas, as observations used for statistical analysis of star formation in disc galaxies have spatial resolutions about some $10^{2} \mathrm{pc}$, compared to our $\sim 30 \mathrm{pc}$. The star formation rate of a given active region is put into relation with a larger volume that contains besides the dense star-forming gas large amounts of ambient, inactive gas. Since the tracers have a certain lifetime, observationally measured star formation rates are temporal averages as well, while our data follow the instantaneous star formation rate. However, the shift of the position of the knee in the $\dot{\Sigma}_{\mathrm{SF}}-\Sigma_{\mathrm{H}_{\mathrm{I}} \mathrm{H}_{2}}$ space with coarsening is a combination of resolution effects and the already discussed effects of stellar to gaseous mass ratio and the metallicity in the dense gas of the discs. Within a resolution element the fraction of gas that is not directly involved in the star formation process increases with decreasing resolution due to averaging. The high-density tail in $\dot{\Sigma}_{\mathrm{SF}}-\Sigma_{\mathrm{HI}+\mathrm{H}_{2}}$ space indicating a correlation of star formation with gas density becomes therefore less pronounced in case of coarser resolution.

\subsubsection{Turbulence}

The main factor that determines the star formation rate $\dot{\rho}_{\mathrm{SF}}$ is the estimated amount of shielded $\mathrm{H}_{2}$. The molecular fraction of the cold phase $f_{\mathrm{H}_{2}}$ is computed by finding the location of the photodissociation front in a spherical clump of size $l_{\mathrm{c}}$ and density $\rho_{\mathrm{c}, \mathrm{pa}}$. The turbulent state has a major impact on these quantities via two competing effects. First, turbulent motions boost the production rate of $\mathrm{H}_{2}$ via local density enhancements of the cold phase. Secondly the turbulent contribution dominates the effective pressure in the cold phase and may play a significant role in the warm-phase pressure as well. For higher $K$ the difference between the phase densities $\rho_{\mathrm{c}, \mathrm{pa}} \geq \rho$ and $\rho_{\mathrm{w}, \mathrm{pa}} \leq \rho$ becomes smaller, which partially counteracts the boost of the production rate. The interplay between these processes determines the minimum $K$ for star formation. In the left panel of Fig. 10 one can see that in the ref run star formation is strongly suppressed for $K>10^{3} \mathrm{~km}^{2} \mathrm{~s}^{-2}$. For high $K$ also the impact of feedback becomes important. Since feedback causes the gas 


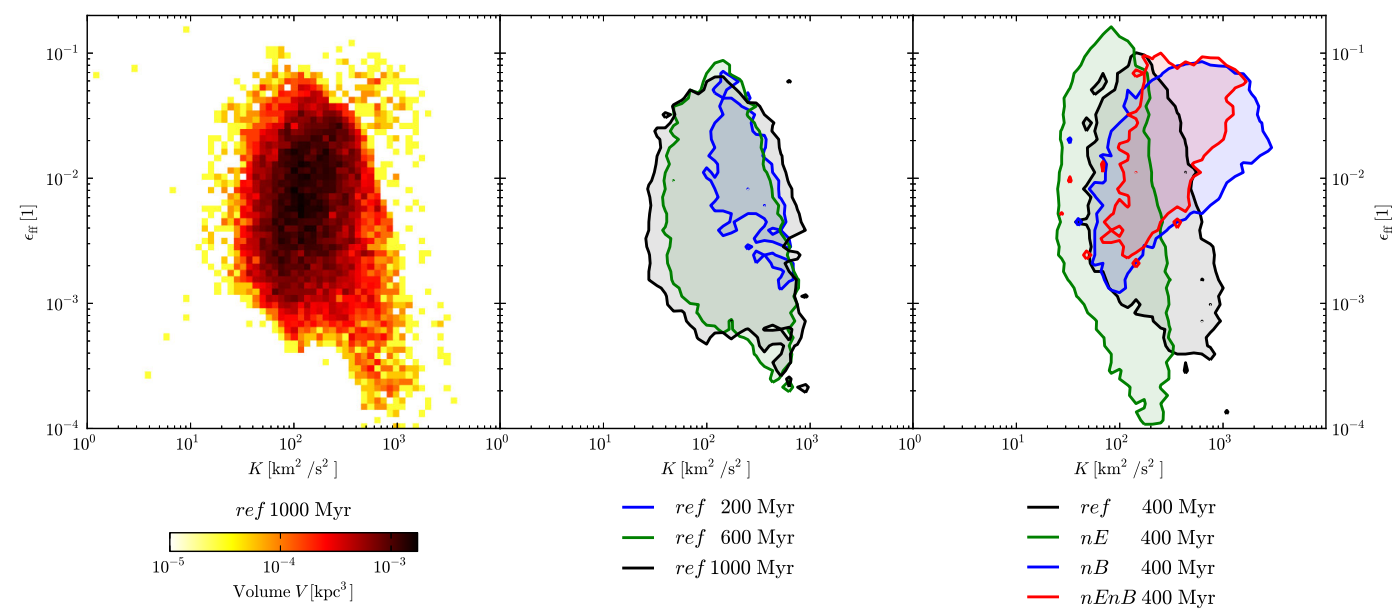

Figure 10. Star formation efficiency $\epsilon_{\mathrm{ff}}$ over $K$. Left panel: phase plot for the central region of the disc in the ref run. Middle and right panels: occupied volume $V_{\text {bin }}>3 \times 10^{-4} \mathrm{kpc}^{3}$ contours of phase diagrams at different times in the ref run, and at $0.4 \mathrm{Gyr}$ in the different runs. Plots and colours are arranged as in Fig. 6 .

to expand, however, there is very little high-density gas that is also strongly turbulent. This explains the relatively narrow range around $K \simeq 100 \mathrm{~km}^{2} \mathrm{~s}^{-2}$ in which star formation actually occurs, which corresponds to a velocity dispersion around $\simeq 10 \mathrm{~km} \mathrm{~s}^{-1}$ or a turbulent RMS Mach number of about 10, consistent with observations (Shetty et al. 2012).

The enrichment of the gas with metals lowers the minimum $K$ for which gas of a given density can become molecular. This effect can be seen in the middle panel of Fig. 10. The degree of enrichment differs throughout the disc, and hence, star formation is possible for a broader range of $K$.

The evolution of an individual star-forming region is easily understood by following its path in the $\epsilon_{\mathrm{ff}}-K$ diagram. Starting at the minimal $K$ for star formation, accretion, collapse, Lyman-feedback, and phase separation rapidly produce turbulence until the production is balanced by dissipation. Simultaneously $\dot{\rho}_{\mathrm{SF}}$ and $\epsilon_{\mathrm{ff}}$ increase and eventually reach a self-regulated state. This regime is associated with the most densely populated area in $\epsilon_{\mathrm{ff}}-K$ space around $K \simeq$ $10^{2} \mathrm{~km}^{2} \mathrm{~s}^{-2}$. But once SNe begin to dominate the thermal evolution locally, density, $\epsilon_{\mathrm{ff}}$, and $\dot{\rho}_{\mathrm{SF}}$ reached their peak values and subsequently decline. Comparing the contours of the runs with $\epsilon_{\mathrm{SN}}=0$ to those with $\epsilon_{\mathrm{SN}}=0.085$ in Fig. 10, it appears that stars are formed in more turbulent environments in the latter case. This reflects the enhancement of $K$ by SN feedback. Switching the hot phase treatment off reduces the impact of thermal feedback, as all thermal SN feedback energy is instantly mixed into the dense warm gas. Since much stronger feedback is needed to dilute the gas, the gaseous disc is clumpier and the clumps tend to be denser in this case. This increases both the star formation efficiency and $K$ as shown in the right panel of Fig. 10. Once the feedback begins to dominate in a clump, the subsequent expansion is much faster without hot phase treatment, and hence, star formation with low $\epsilon_{\mathrm{ff}}$ in strongly turbulent gas is cut off.

\subsection{Drivers of turbulence}

In the MIST model implementation we consider two groups of sources of SGS turbulence. First there is the production of small- scale turbulence by large-scale shear and compression. The corresponding source term $\Pi_{\mathrm{SGS}}$ in equation (10) may be both positive or negative, indicating whether small-scale turbulence is driven by resolved motions (direct cascade) or the other way around (inverse cascade). The internal sources

$\Pi_{\mathrm{int}}:=\Pi_{\mathrm{SN}}+\Pi_{\mathrm{TI}}$

are specific to MIST. The physical processes modelled here are the phase separation due to thermal instability $\left(\Pi_{\mathrm{TI}}\right.$, equation 21$)$, and small-scale motions caused by SNe bubbles and instabilities in their blast waves $\left(\Pi_{\mathrm{SN}}\right.$, equation 22$)$. Our model enables us to disentangle the contributions to the total production rate $\Pi_{\text {tot }}:=$ $\Pi_{\mathrm{SGS}}+\Pi_{\text {int }}$ from the turbulent cascade and internal sources to answer the question, which processes are most relevant in which regime.

We can distinguish four regimes, as demonstrated in Fig. 11 for the ref run:

(i) $K \lesssim 10 \mathrm{~km}^{2} \mathrm{~s}^{-2}$ : negative $\Pi_{\mathrm{SGS}}$ indicate expanding environments. In this case, it is the signature of infalling dilute gas. The material found in this regime is most likely to fall freely towards some dense clump. $\Pi_{\text {int }} \lesssim-\Pi_{\mathrm{SGS}}$ is caused by the enforcement of the floor $K_{\min }=0.05 \mathrm{~km}^{2} \mathrm{~s}^{-2} \ll e_{\mathrm{c}} .5$

(ii) $10 \mathrm{~km}^{2} \mathrm{~s}^{-2} \lesssim K \lesssim 100 \mathrm{~km}^{2} \mathrm{~s}^{-2}$ : turbulence is mainly supported through the turbulent cascade $\Pi_{\mathrm{SGS}}$. A sub-dominant contribution is $\Pi_{\mathrm{TI}}$. This regime is typical for star-forming clumps in their early evolutionary stages.

(iii) $100 \mathrm{~km}^{2} \mathrm{~s}^{-2} \lesssim K \lesssim 1000 \mathrm{~km}^{2} \mathrm{~s}^{-2}: \Pi_{\mathrm{SGS}}$ is the main driver of unresolved turbulence in low-density environments, but $\Pi_{\text {int }}$ dominates and $\Pi_{\mathrm{SGS}}$ becomes negative in dense environments (see Fig. 12). The latter case indicates an expanding environment. For greater $K$ the internal sources $\Pi_{\text {int }}$ become increasingly dominated by $\Pi_{\mathrm{SN}}$. Star-forming regions in late evolutionary stages reside in this regime.

${ }^{5} \mathrm{~A}$ minimum level of turbulence is needed for the implementation of the SF11-model to work properly, as $\Pi_{\mathrm{SGS}}$ depends directly on the instant value of $K$ (see equations 10 and 8 ). 


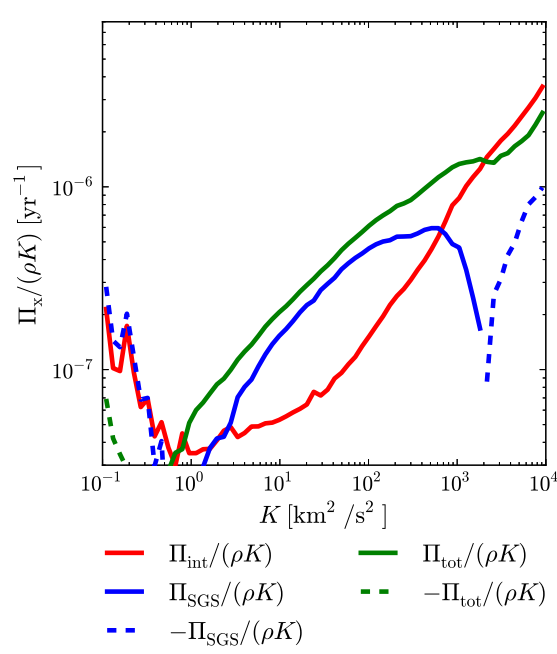

Figure 11. Volume-weighted mean of inverse production time-scales of turbulent sub-grid energy $\Pi /(\rho K)$ over specific unresolved turbulent energy $K$ in the star-forming part of the disc from the ref run after $1 \mathrm{Gyr}$. The inverse time-scale $\Pi_{\mathrm{SGS}} /(\rho K)$ of production by resolved motions via the turbulent stress tensor is shown in blue. The inverse time-scale $\Pi_{\text {int }} /(\rho K):=\left(\Pi_{\mathrm{TI}}\right.$ $\left.+\Pi_{\mathrm{SN}}\right) /(\rho K)$ of the non-adiabatic MIST-sources is printed in red, and the inverse time-scale $\Pi_{\text {tot }} /(\rho K):=\left(\Pi_{\text {int }}+\Pi_{\mathrm{SGS}}\right) /(\rho K)$ of the total small-scale turbulent energy production. A solid line indicates positive, and a dashed line negative values.

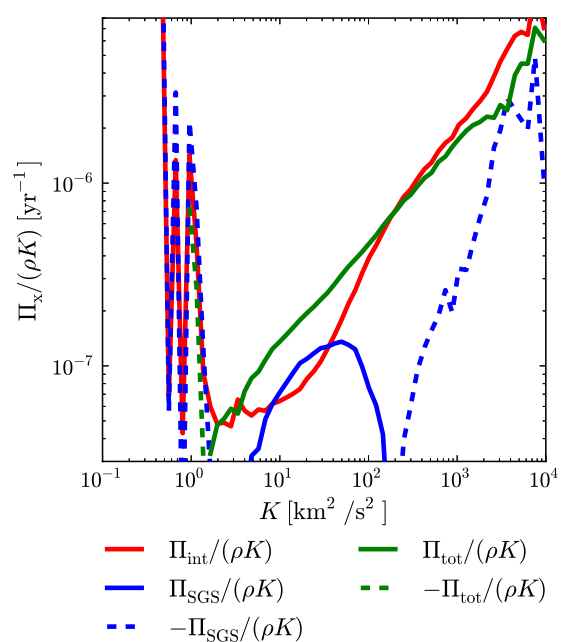

Figure 12. Volume-weighted mean of inverse production time-scales of turbulent sub-grid energy $\Pi /(\rho K)$ over specific unresolved turbulent energy $K$ in dense $\left(\rho>0.032 \mathrm{M}_{\odot} \mathrm{pc}^{-3}\right)$ areas of the disc from the ref run after $1 \mathrm{Gyr}$. Line colours and styles are arranged as in Fig. 11

(iv) $K \gtrsim 1000 \mathrm{~km}^{2} \mathrm{~s}^{-2}$ : SNe feedback dominates not only the thermal evolution but also all other contributions to turbulence production. The gas in this regime is hot and rapidly expanding.

The power-law behaviour $\Pi_{\text {tot }} /(\rho K) \propto K^{\sim 0.5}$ for intermediate $K$ indicates that $\Pi_{\text {tot }}$ is, on average, balanced by turbulent dissipation $\rho \epsilon_{\mathrm{SGS}} \propto \rho K^{1.5}$. While $\Pi_{\mathrm{TI}}$ is slightly enhanced in the absence of $\Pi_{\mathrm{SN}}$ (i.e. in the $\mathrm{nE}$ and $\mathrm{nEnB}$ run), $\Pi_{\mathrm{SGS}}$ remains roughly unchanged

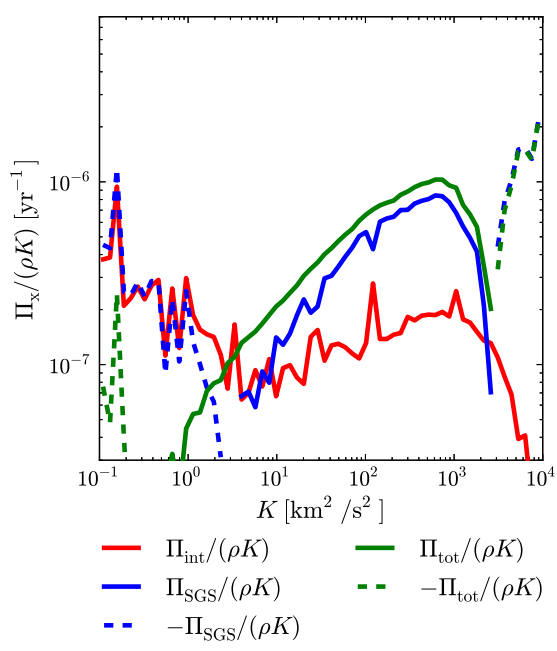

Figure 13. Volume-weighted mean of inverse production time-scales of turbulent sub-grid energy $\Pi /(\rho K)$ over specific unresolved turbulent energy $K$ in the star-forming part of the disc from the $\mathrm{nE}$ run after $1 \mathrm{Gyr}$. Line colours and styles are arranged as in Fig. 11.

(see Fig. 13). In this case the low $K$-regime is extended up to $K \lesssim$ $50 \mathrm{~km}^{2} \mathrm{~s}^{-2}$. A further consequence of $\Pi_{\mathrm{SN}}=0$ is the lack of effective turbulence production for $K>10^{3} \mathrm{~km}^{2} \mathrm{~s}^{-2}$.

\subsection{Resolution study}

With decreasing resolution less of the dynamics in the discs is resolved. In particular, the relative importance of the stellar feedback compared to gravity is reduced, as the feedback energy is deposited in a larger volume. This leads to more clumpy discs in the lowresolution runs (see Fig. 14). The resulting gaseous structures like knots and connecting features are much more extended and more massive than one would expect from the ratio of resolutions alone. BS12 have shown that the size of the reference volume, which corresponds to a numerical resolution element, does not affect the equilibrium solutions. However, the time-scales related to an evolution from an arbitrary state towards equilibrium in the BS12 model are considerably longer for larger $\Delta$, which is caused by additional turbulent modes in a larger reference volume.

This effect could be compensated for by adjusting $\epsilon_{\mathrm{SN}}, e_{\mathrm{h}}$, and $\tau_{\mathrm{h}}$. The physical reasoning is that an increased fraction of the energy released by $\mathrm{SNe}$ is present in the form of motions on larger spatial scales driven by the expanding $\mathrm{SNe}$ bubbles which provide additional pressure support.

Despite the differences in the disc structure, the global star formation rates in the ref and lres5 run are in good agreement with each other (see Fig. 15), although the initial transient phase in the lres5 run ( $\Delta \simeq 60 \mathrm{pc}$ ) lasts longer than in the ref run $(\Delta \simeq 30 \mathrm{pc})$. In both runs about $\sim 30$ per cent of the initial mass were transformed into stars after $1 \mathrm{Gyr}$. Star formation in the lres 4 run $(\Delta \simeq 120 \mathrm{pc})$ is less efficient, such that only $\sim 15$ per cent of the mass was turned into stars after 1 Gyr. Due to lack of resolution and the effect discussed above, the relevant structures for star formation either are not sufficiently resolved or simply do not form.

Regardless of the differences between the runs, the star formation versus density relations, as shown in Fig. 16 for $\dot{\Sigma}_{\mathrm{SF}}$ over $\Sigma_{\mathrm{H}_{2}}$ and in Fig. 17 for $\dot{\Sigma}_{\mathrm{SF}}$ over $\Sigma_{\mathrm{H}_{\mathrm{I}} \mathrm{H}_{2}}$, stay roughly unchanged, which is a 
3422

\section{H. Braun et al.}

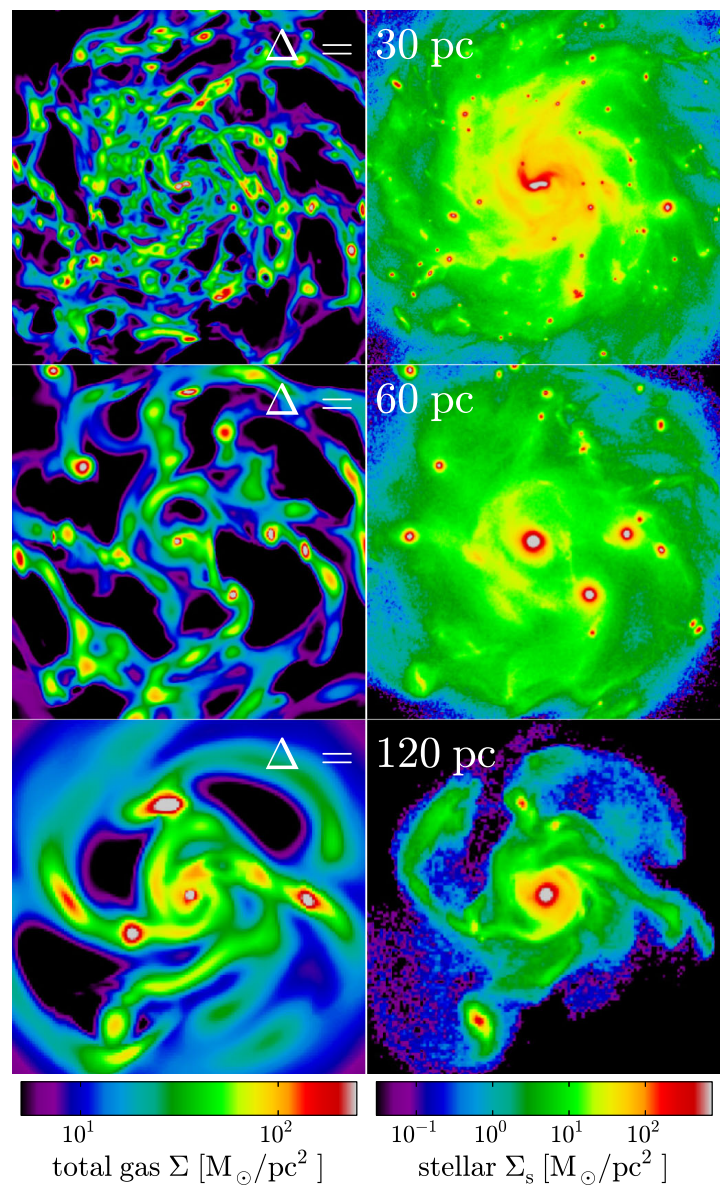

time: 1 Gyr $\mid$ field of view $15 \times 15 \mathrm{pc}$

Figure 14. Comparison of total gas surface density $\Sigma$ in the left column and the stellar surface density $\Sigma_{\mathrm{s}}$ in the right column between the runs of different effective resolution (from top to bottom: ref, lres5, lres4) 1 Gy after start of simulation

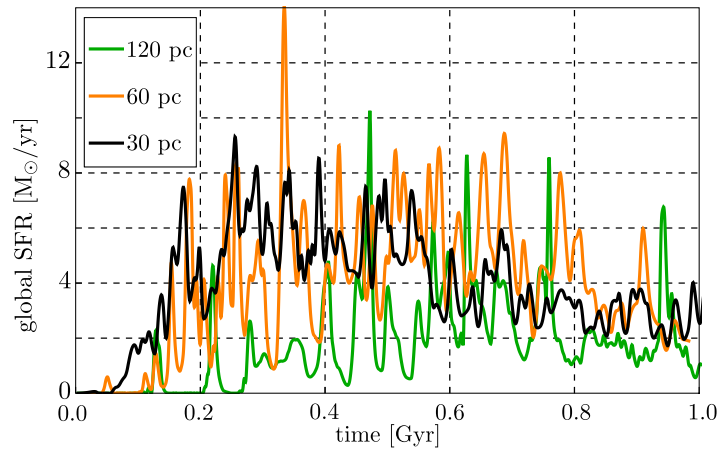

Figure 15. Global star formation rate $\dot{M}_{\mathrm{SF}}$ over simulation time for the different runs lres4, lres5, and ref in green, orange, and black, respectively.

MNRAS 442, 3407-3426 (2014)

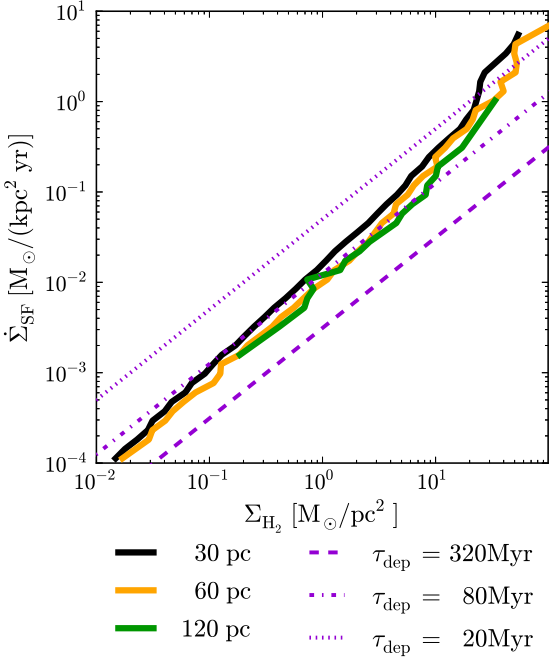

Figure 16. Star formation column density $\dot{\Sigma}_{\mathrm{SF}}$ over $\mathrm{H}_{2}$ column density $\Sigma_{\mathrm{H}_{2}}$ for runs with different numerical resolution: lres4, lres5, and ref in green, orange, and black, respectively.

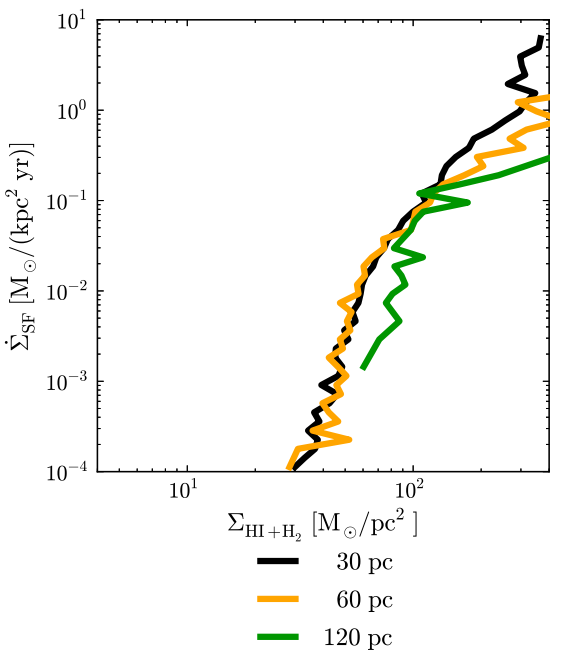

Figure 17. Star formation column density $\dot{\Sigma}_{\mathrm{SF}}$ over $\mathrm{H}_{\mathrm{I}}+\mathrm{H}_{2}$ column density $\Sigma_{\mathrm{HI}+\mathrm{H}_{2}}$ for runs with different numerical resolution: lres4, lres5, and ref in green, orange, and black, respectively.

consequence of the internal regulation of MIST. The slight shift in the case of the plots with $\Delta=120$ pc in Figs 9 and 17 is caused by the generally lower star formation rate in the lres 4 run compared to the ref run.

\subsection{Impact of subgrid-scale model}

Fig. 18 shows that the stellar discs in the runs without MIST (sSF and SSF2) are dominated by a few very massive stellar clusters after 400 Myr. The gas follows the distribution of stars and is consequently concentrated in the stellar clusters. Star formation occurs only in those places, since there is no high-density gas elsewhere. 


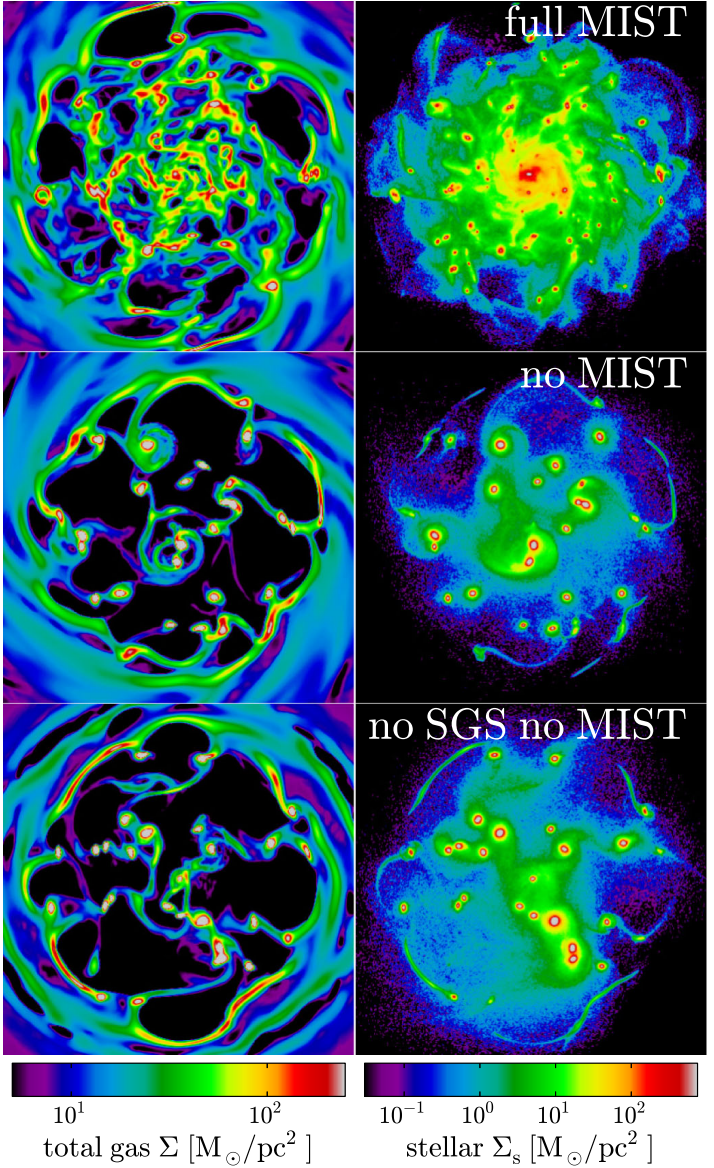

time : $400 \mathrm{Myr}$ | field of view $15 \times 15 \mathrm{pc}$

Figure 18. Comparison of total gas surface density $\Sigma$ in the left column and the stellar surface density $\Sigma_{\mathrm{s}}$ in the right column between the runs using different ISM models (from top to bottom: ref, sSF, sSF2) $400 \mathrm{Myr}$ after start of simulation.

Clearly, the Truelove et al. (1997) criterion is not fulfilled within those clusters, as neither SGS turbulence energy nor SNe energy feedback can support the gas sufficiently in both runs. The SGS model is necessary to keep the gas (and in the consequence the stars) from clustering too much in order to obtain a realistically smooth and flocculent disc.

The global star formation rate of a few $\mathrm{M}_{\odot} / \mathrm{yr}$ in sSF and sSF2 is comparable to the ref run (see Fig. 19). This is a consequence of the choice of the parameters in the simplified star formation model $\left(\varepsilon_{\mathrm{sSF}}=0.01, \rho_{\mathrm{SSF}, \min }=50 \mathrm{~m}_{\mathrm{H}} \mathrm{cm}^{-3}\right.$ and $\left.T_{\mathrm{sSF}, \max }=1.5 \times 10^{4} \mathrm{~K}\right)$, which are chosen to match the average star formation properties in the runs with MIST. However, both runs with the simplified model lack the strong variations on time-scales between 10 and $30 \mathrm{Myr}$ seen in runs with MIST. As pointed out in Sections 4.1 and 4.2 .1 , the variations are related to the life cycle of individual starforming regions. The $\mathrm{SNe}$ feedback disrupting the cold dense gas after a phase of intense star formation limits the lifetime of an active region in the MIST runs. The lifetime of these regions is not limited in the non-MIST runs, as the feedback energy is radiated away

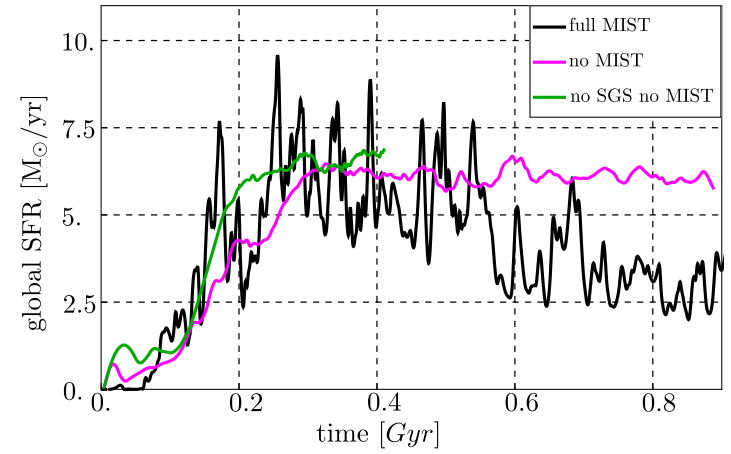

Figure 19. Global star formation rate $\dot{M}_{\mathrm{SF}}$ over simulation time for the different runs ref, sSF, and sSF2 in black, purple, and green, respectively.

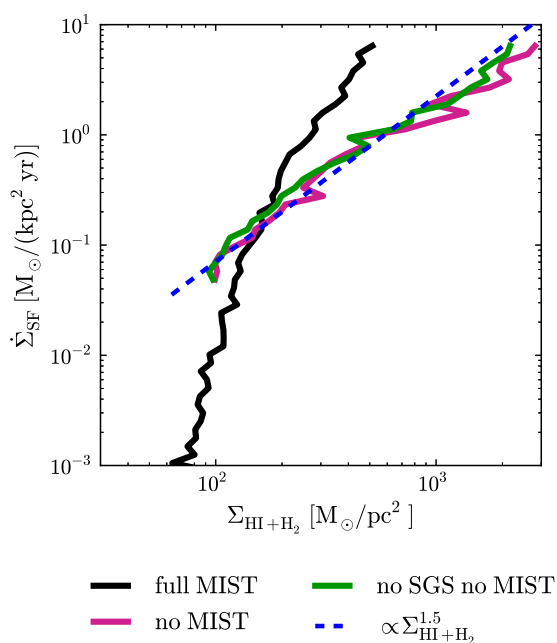

Figure 20. Star formation column density $\dot{\Sigma}_{\mathrm{SF}}$ over $\mathrm{H}_{\mathrm{I}}+\mathrm{H}_{2}$ column density $\Sigma_{\mathrm{H} 1+\mathrm{H}_{2}}$ for the runs ref, sSF, and sSF2 in black, purple, and green, respectively.

before it could affect the gas. This is known as the so-called 'overcooling' problem. Moreover, the stellar particles are not inserted with peculiar velocities, representing the unresolved motions of the star-forming gas (see Section 2.3.1). These differences in the treatment of star formation and feedback result in the formation of large strongly bound clusters, in which continuous star formation is fuelled by the accumulation of gas through accretion and mergers into even larger clusters. This is a runaway process.

After an initial transient phase the global star formation rate reaches a plateau around $6 \mathrm{M}_{\odot} / \mathrm{yr}$ (see Fig. 19). Due to the stabilizing effect of the SGS turbulence energy the transient phase in sSF lasts 50 Myr longer than in sSF2. The gradual decline of the star formation rate seen in runs with MIST due to metal enrichment (see Section 4.2.1) does not occur in $\mathrm{SSF}$ and $\mathrm{sSF} 2$, as neither $\varepsilon_{\mathrm{sSF}}$ nor $\rho_{\mathrm{SSF}, \min }$ depend on metals. The KS relation, depicted in Fig. 20, shows a tight correlation with the expected slope of $\dot{\Sigma}_{\mathrm{SF}} \propto \Sigma^{1.5}$. 


\section{H. Braun et al.}

\section{DISCUSSION AND CONCLUSIONS}

In this paper we introduced MIST, which is a SGS model based on the semi-analytical BS12 model (Braun \& Schmidt 2012) for the turbulent multi-phase ISM. We implemented MIST into the code Nyx (Almgren et al. 2013) to run adaptively refined LES of IDG with different stellar feedback parameters. For the first time, a complete treatment of the numerically unresolved turbulence energy via the SGS model of SF11 is applied in such simulations. The star formation recipe follows Krumholz et al. (2009) and PN11. In our fiducial galaxy model, supernova feedback produces both SGS turbulence energy and heat. Since the injected heat would instantly cool away in dense environments, we suppress cooling in the hot gas produced by feedback over a time-scale that is given by the typical size and expansion velocities of hot $\mathrm{SN}$ bubbles in the ISM. Comparison runs demonstrate that the following properties of star formation in disc galaxies are reproduced if both turbulent and thermal feedback are applied:

(i) an average total gas star formation efficiency $\epsilon_{\mathrm{ff}} \simeq 0.01$.

(ii) $\epsilon_{\mathrm{ff}}$ is enhanced in dense environments

(iii) a galactic depletion time-scale $\tau_{\mathrm{dep}} \sim 0.3 \ldots 1 \mathrm{Gyr}$ in a gasrich galaxy.

(iv) a linear relationship between $\dot{\Sigma}_{\mathrm{SF}}$ and $\Sigma_{\mathrm{H}_{2}}$.

(v) a lifespan of molecular clouds $t_{\mathrm{GMC}} \sim 10, \ldots, 30 \mathrm{Myr}$.

(vi) a velocity dispersion in star-forming regions $\sigma_{\mathrm{SF}} \simeq$ $10 \mathrm{~km} \mathrm{~s}^{-1}$.

We observe three modes of star formation in our simulations. First, stars are formed in the metal-poor gas that occurs in isolated clouds. In this material, the threshold density for star formation is rather high. Therefore, the gas is quickly turned into stars and the ensuing feedback disrupts the cloud violently in the simulation with full feedback. Subsequent dispersion of the gas effectively quenches star formation, as the expanding shell is diluted below the threshold density. Typically, a stellar cluster is left behind that survives longer than the original gas cloud. Eventually, the stars are dispersed in the disc's potential or merge into more stable and massive clusters. With increasing amounts of metals in the gas, a different mode of star formation emerges. Owing to the decreasing threshold density, active star formation is possible in more extended regions of lower density. Although star formation is still quenched by feedback in these regions, waves of star formation propagate through the metalrich inner parts of the disc. Since the feedback is less violent, the winds launched in this mode are slower. The third mode of star formation is hosted by massive stellar clusters that locally dominate the gravitational potential. They are formed via mergers of the clusters generated in metal-poor star-forming regions. Once they are massive enough, they begin to accrete gas from their surroundings, and consequently host star formation. Depending on their mass and the chosen feedback parameters, star formation is continuous or intermittent. The massive clusters are candidates for globular cluster progenitors, although they continue to grow and slowly spiral towards the centre in our isolated disc simulation, where they merge into the central stellar agglomeration. In a cosmological galaxy simulation, mergers could interrupt this process by kicking the clusters out of the disc plane and thereby cutting them off of gas supply. Moreover, it is possible that late feedback from SNe of type Ia (Agertz et al. 2013) has an impact on the star formation induced by clusters. In the current implementation, stellar populations older than roughly $40 \mathrm{Myr}$ are quiescent. Taking SNe Ia into account, the period of active feedback might increase to a few $100 \mathrm{Myr}$. Although this type of feedback is much less intense than the feedback caused by $\mathrm{SNe}$ II, it might prevent the accretion of gas in dense stellar clusters. Also so-called 'early feedback' (e.g. see Stinson et al. 2013) in the form of turbulent feedback due to stellar winds from massive stars might make residual stellar clusters more prone to disruption, since the velocity dispersion of the stars would be enhanced.

For all of our runs using MIST, we found a robust, almost linear relationship between shielded molecular gas and the star formation rate, which is a consequence of self-regulatory processes in MIST. This kind of relation also appears in the semi-analytic model (BS12) and in observations (Bigiel et al. 2011). The related $\mathrm{H}_{2}$-consumption time-scale of 40 to $80 \mathrm{Myr}$ is in good agreement with observations of actively star-forming clouds (Murray 2011). The global gas depletion time-scale of about $0.5-1 \mathrm{Gyr}$, which is inferred from the mean star formation rate in the simulation, is in agreement with the observed gas depletion in gas-rich galaxies (Daddi et al. 2010). The gradual increase of this time-scale suggests that depletion times of a few Gyr could be reached in later evolutionary stages, comparable to those observed for local galaxies. The gas consumption timescale inferred from the relation between $\dot{\Sigma}_{\mathrm{SF}}$ and $\Sigma_{\mathrm{HI}+\mathrm{H}_{2}}$ is also in agreement with observations of gas-rich galaxies. These results confirm our method of computing the star formation rate. However, the amount of molecular gas following from our model is generally too low to be consistent with observations. The missing $\mathrm{H}_{2}$ is a consequence of using a Strömgren-like ansatz to obtain an equilibrium solution instead of following the chemical evolution. Although this allows us to reliably estimate the amount of molecular gas that resides in shielded areas, a large fraction of the $\mathrm{H}_{2}$ mass would be found in surrounding regions if detailed chemical reaction networks were computed in the cold and warm phases. As the star formation rates in our simulation reproduce observations reasonably well, it appears that the shielding from radiation, and hence the lack of heating, is the major factor that controls star formation.

While the star formation efficiency $\epsilon_{\mathrm{PN}}$ with respect to the shielded gas is almost constant for all star-forming regions, regardless of density and metallicity, the efficiency $\epsilon_{\mathrm{ff}}$ with respect to the total gas density does vary due to the impact of metals and density on the shielded $\mathrm{H}_{2}$ content. This results in an enhanced efficiency $\epsilon_{\mathrm{ff}}$ in regions with high star formation rate, contrary to the sometimes employed constant efficiency parameters (e.g. Agertz et al. 2013). Since the production of $\mathrm{H}_{2}$ strongly increases with the density of the unresolved cold-gas clumps, which is enhanced by turbulence via the dependence of the cold-gas density PDF on the turbulent Mach number in our model, a minimal level of SGS turbulence energy is necessary for star formation. Stellar feedback, which is associated with very high SGS turbulence energy, reduces star formation. Consequently, star formation occurs only for an intermediate range of SGS turbulence energy, centred around a peak value that roughly corresponds to a velocity dispersion of $10 \mathrm{~km} \mathrm{~s}^{-1}$, comparable to observed values (Shetty et al. 2012). Apart from feedback, the turbulent cascade is an important source for building up the moderate level of turbulence in star-forming regions before SN feedback becomes dominant.

While Agertz et al. (2013) make efforts towards accounting for all relevant feedback mechanisms - these are stellar winds from massive stars, radiation pressure, SNe II, SNe Ia, and mass loss by mass-poor stars - in a realistic fashion by taking age and metallicity of a stellar population into account and using appropriate application channels and schemes for each of them reflecting their physical impact on the ISM, their star formation recipe is relatively simple. In contrast to their approach, we focus on a more elaborate way to describe the sub-resolution structure and processes in the ISM. The 
non-thermal pressure - actually the trace of the turbulent stress tensor - helps to stabilize gas against gravity particularly in cold and dense environments. The SGS-energy allows us to collect the effect of subresolution-scale motions excited by $\mathrm{SNe}$, which is our counterpart to the momentum feedback in Agertz et al. (2013). While over-cooling is usually avoided by suppressing all cooling for period of more than $10 \mathrm{Myr}$, the combined effect of non-thermal pressure and turbulent feedback reduces the need for delayed cooling in areas of active $\mathrm{SNe}$ feedback in our simulations.

MIST coupled to an SGS model is suitable for the use in cosmological zoom-in simulations with effective resolutions of about 10 to $100 \mathrm{pc}$ without substantial modifications. However, the current model framework of MIST has to be adjusted to treat extremely metal-poor gas and the radiation background consistently. Apart from that the incorporation of reaction networks to track the actual molecular content in the different gas phases and of additional stellar feedback mechanisms like stellar winds from massive stars and $\mathrm{SNe}$ Ia will further enhance the model.

\section{ACKNOWLEDGEMENTS}

HB was financially supported by the CRC 963 of the German Research Council. The work of AA was supported by the SciDAC FASTMath Institute, funded by the Scientific Discovery through Advanced Computing (SciDAC) program funded by U.S. Department of Energy. HB, WS and JCN acknowledge financial support by the German Research Council for visits at LBNL. We thank Hsiang-Hsu Wang for discussions on the initial conditions of idealized IDG. The simulations presented in this article were performed on the SuperMUC of the LRZ (project pr47bi) in Germany. We also acknowledge the yt toolkit by Turk et al. (2011) that was used for our analysis of numerical data. We owe thanks to the referee B. Robertson for a careful and helpful report that helped us to improve this paper.

\section{REFERENCES}

Agertz O., Lake G., Teyssier R., Moore B., Mayer L., Romeo A. B., 2009, MNRAS, 392, 294

Agertz O., Kravtsov A. V., Leitner S. N., Gnedin N. Y., 2013, ApJ, 770, 25 Almgren A. S., Bell J. B., Lijewski M. J., Lukić Z., Van Andel E., 2013, ApJ, 765, 39

Benincasa S. M., Tasker E. J., Pudritz R. E., Wadsley J., 2013, ApJ, 776, 23 Bigiel F., Leroy A., Walter F., Brinks E., de Blok W. J. G., Madore B., Thornley M. D., 2008, AJ, 136, 2846

Bigiel F. et al., 2011, ApJ, 730, L13

Blanc G. A., Heiderman A., Gebhardt K., Evans N. J., II, Adams J., 2009, ApJ, 704, 842

Blitz L., Fukui Y., Kawamura A., Leroy A., Mizuno N., Rosolowsky E., 2007, in Reipurth B., Jewitt D., Keil K., eds, Protostars and Planets V. University of Arizona Press, Tucson, p. 81

Booth C. M., Agertz O., Kravtsov A. V., Gnedin N. Y., 2013, ApJ, 777, L16 Bournaud F. et al., 2011, ApJ, 730, 4

Braun H., Schmidt W., 2012, MNRAS, 421, 1838 (BS12)

Chabrier G., 2001, ApJ, 554, 1274

Daddi E. et al., 2010, ApJ, 713, 686

Dobbs C. L., Pringle J. E., 2013, MNRAS, 432, 653

Elmegreen B. G., Burkert A., 2010, ApJ, 712, 294

Evans N. J., II, 2008, in Knapen J. H., Mahoney T. J., Vazdekis A., eds, ASP Conf. Ser. Vol. 390, Pathways Through an Eclectic Universe. Star Formation in Molecular Clouds? Astron. Soc. Pac., San Francisco, p. 52

Evans N. J., II et al., 2009, ApJS, 181, 321

Federrath C., Klessen R. S., 2013, ApJ, 763, 51
Federrath C., Roman-Duval J., Klessen R. S., Schmidt W., Mac Low M., 2010, A\&A, 512, A81

Ferland G. J., Korista K. T., Verner D. A., Ferguson J. W., Kingdon J. B., Verner E. M., 1998, PASP, 110, 761

Gao Y., Solomon P. M., 2004, ApJ, 606, 271

Genel S., Dekel A., Cacciato M., 2012, MNRAS, 425, 788

Genzel R. et al., 2010, MNRAS, 407, 2091

Gnedin N. Y., Tassis K., Kravtsov A. V., 2009, ApJ, 55

Gómez G. C., Cox D. P., 2002, ApJ, 580, 235

Hennebelle P., Chabrier G., 2011, ApJ, 743, L29

Herrera C. N., Boulanger F., Nesvadba N. P. H., 2011, A\&A, 534, A138

Hopkins P. F., Kereš D., Murray N., 2013, MNRAS, 432, 2639

Hughes A. et al., 2013, ApJ, 779, 44

Iwasaki K., Inutsuka S.-i., 2014, ApJ, 784, 115

Kennicutt R. C., Jr, 1998, ApJ, 498, 541

Kennicutt R. C., Evans N. J., 2012, ARA\&A, 50, 531

Kim W.-T., Ostriker E. C., 2007, ApJ, 660, 1232

Kim W.-T., Ostriker E. C., Stone J. M., 2003, ApJ, 599, 1157

Klessen R. S., Hennebelle P., 2010, A\&A, 520, A17

Kraljic K., Bournaud F., Martig M., 2012, ApJ, 757, 60

Kritsuk A. G., Norman M. L., 2002, ApJ, 569, L127

Krumholz M., Burkert A., 2010, ApJ, 724, 895

Krumholz M. R., McKee C. F., 2005, ApJ, 630, 250

Krumholz M. R., Thompson T. A., 2012, ApJ, 760, 155

Krumholz M. R., McKee C. F., Tumlinson J., 2009, ApJ, 699, 850

Lada C. J., Lombardi M., Alves J. F., 2010, ApJ, 724, 687

Leroy A. K., Walter F., Brinks E., Bigiel F., de Blok W. J. G., Madore B., Thornley M. D., 2008, AJ, 136, 2782

Leroy A. K. et al., 2013, AJ, 146, 19

McKee C. F., Ostriker E. C., 2007, ARA\&A, 45, 565

Miura R. E. et al., 2012, ApJ, 761, 37

Monaco P., Murante G., Borgani S., Dolag K., 2012, MNRAS, 421, 2485

Munshi F. et al., 2013, ApJ, 766, 56

Murante G., Monaco P., Giovalli M., Borgani S., Diaferio A., 2010, MNRAS, 405,1491

Murray N., 2011, ApJ, 729, 133

Navarro J. F., Frenk C. S., White S. D. M., 1997, ApJ, 490, 493

Onodera S. et al., 2010, ApJ, 722, L127

Ostriker E. C., Shetty R., 2011, ApJ, 731, 41

Padoan P., Nordlund Å., 2011, ApJ, 730, 40 (PN11)

Padoan P., Haugbølle T., Nordlund Å., 2012, ApJ, 759, L27

Raiteri C. M., Villata M., Navarro J. F., 1996, A\&A, 315, 105

Renaud F. et al., 2013, MNRAS, 436, 1836

Saintonge A. et al., 2013, ApJ, 778, 2

Schmidt M., 1959, ApJ, 129, 243

Schmidt W., Federrath C., 2011, A\&A, 528, A106 (SF11)

Schmidt W. et al., 2014, MNRAS, 440, 3051

Schneider N. et al., 2014, preprint (arXiv e-prints)

Schruba A., Leroy A. K., Walter F., Sandstrom K., Rosolowsky E., 2010, ApJ, 722, 1699

Shetty R., Beaumont C. N., Burton M. G., Kelly B. C., Klessen R. S., 2012, MNRAS, 425, 720

Stilp A. M., Dalcanton J. J., Warren S. R., Weisz D. R., Skillman E., Ott J., Williams B. F., Dolphin A. E., 2013, ApJ, 772, 124

Stinson G., Seth A., Katz N., Wadsley J., Governato F., Quinn T., 2006, MNRAS, 373, 1074

Stinson G. S., Brook C., Macciò A. V., Wadsley J., Quinn T. R., Couchman H. M. P., 2013, MNRAS, 428, 129

Tacconi L. J. et al., 2013, ApJ, 768, 74

Tasker E. J., Tan J. C., 2009, ApJ, 700, 358

Teyssier R., Chapon D., Bournaud F., 2010, ApJ, 720, L149

Truelove J. K., Klein R. I., McKee C. F., Holliman J. H., II, Howell L. H., Greenough J. A., 1997, ApJ, 489, L179

Turk M. J., Smith B. D., Oishi J. S., Skory S., Skillman S. W., Abel T., Norman M. L., 2011, ApJS, 192, 9

Vink J. S., 2011, Ap\&SS, 336, 163

Vink J. S., de Koter A., Lamers H. J. G. L. M., 2000, A\&A, 362, 295

Vollmer B., Beckert T., 2003, A\&A, 404, 21 
3426

\section{H. Braun et al.}

Wada K., Norman C. A., 2001, ApJ, 547, 172

Wada K., Meurer G., Norman C. A., 2002, ApJ, 577, 197

Wang H.-H., Klessen R. S., Dullemond C. P., van den Bosch F. C., Fuch B., 2010, MNRAS, 407, 705

Wise J. H., Abel T., Turk M. J., Norman M. L., Smith B. D., 2012, MNRAS, 427,311
Wolf-Chase G. A., Barsony M., O’Linger J., 2000, AJ, 120, 1467

Wong T., Blitz L., 2002, ApJ, 569, 157

This paper has been typeset from a $\mathrm{T}_{\mathrm{E}} \mathrm{X} / \mathrm{L} \mathrm{T} \mathrm{E}$ file prepared by the author. 


\section{Rückseite:}

Spitzkoppe, Namibia, aufgenommen von Harald Braun in 2014.

Turbulenz wohin man blickt. Sie gibt nicht nur den Wolken ihre charakteristische Form. Die Intrusion des Magmaplumes, dessen oberes Ende vor geraumer Zeit zu jenem Granitblock in der Ferne erstarrte, noch bevor er die Erdoberfläche erreicht hatte, war ein Ergebnis turbulenter Konvektion im Erdmantel. Wind und Wetter haben ihn durch allmähliche Erosion des umliegenden, weniger beständigen Gesteins freigelegt. 


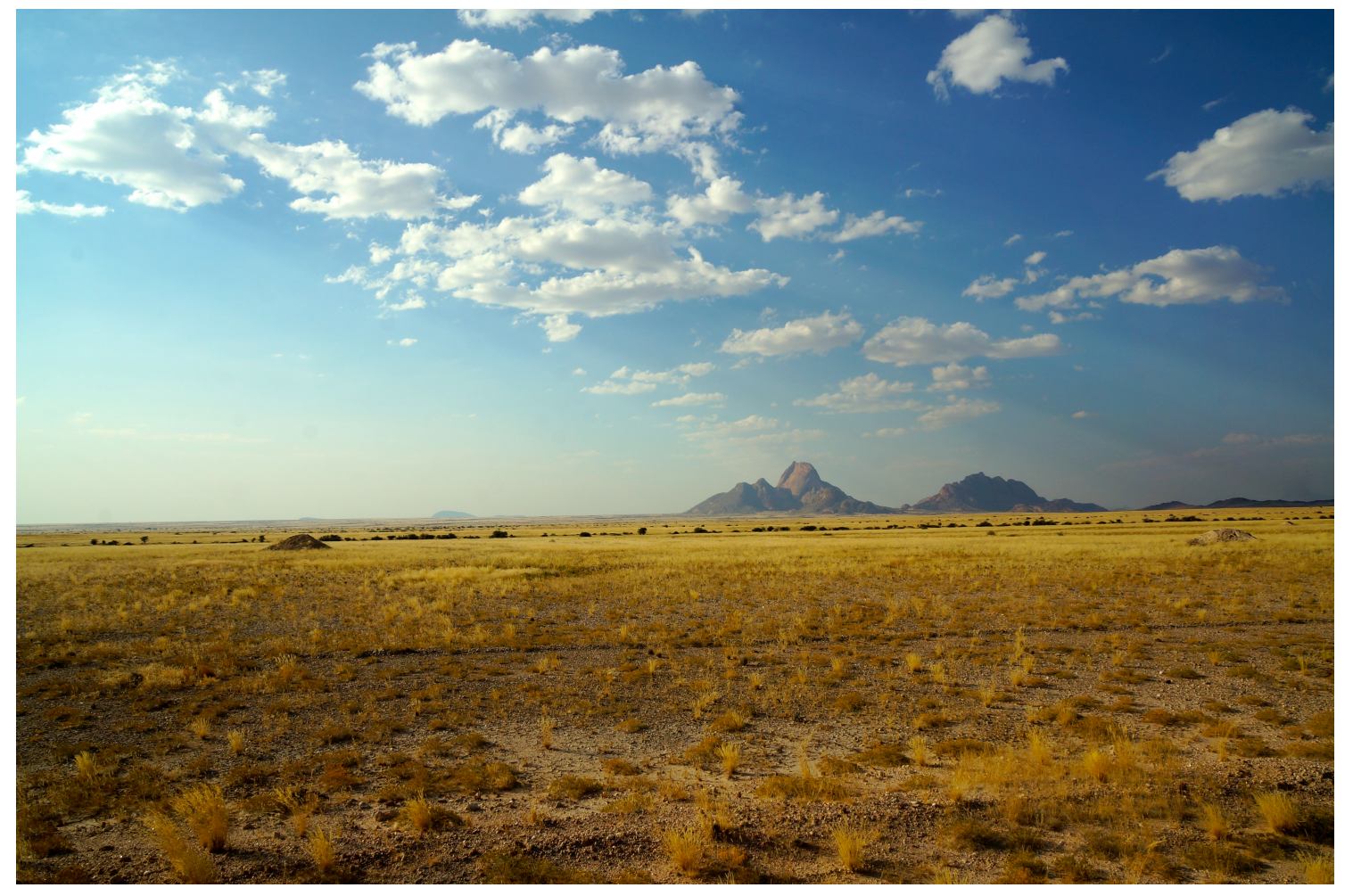

